\title{
The 100-Car Naturalistic Driving Study: A Descriptive Analysis of Light Vehicle-Heavy Vehicle Interactions from the Light Vehicle Driver's Perspective
}




\section{Foreword}

The 100-Car Naturalistic Driving Study was undertaken with the goal of obtaining data on driver performance and behavior in the moments leading up to a crash. This type of data is not available from either of the traditional methods of studying driver behavior in regards to crashes and traffic safety, such as empirical studies and crash databases (e.g., General Estimates System and the Fatality Analysis Reporting System). Crash databases are derived from police accidents reports (PARs) and contain a wealth of data describing the non-controversial facts of the crash such as location, number of vehicles involved, type of crash, and time of day. For a variety of reasons, however, these databases do not provide good insight into the driver behavior and performance leading up to the crash. The empirical method provides a different approach to investigating driver behavior by studying how people drive under various conditions. These studies are usually conducted as highly controlled experiments using instrumented vehicles to obtain a variety of vehicle and driver performance data. Typically, these studies involve drivers operating study test vehicles for a short period of time (i.e., a few hours) in a contrived environment (i.e., either simulator or closed test track).

Naturalistic studies can be used to understand crash causation and driver behavior and supplement information learned through epidemiological and empirical approaches. Naturalistic studies include driver/subjects operating vehicles in their daily lives (e.g., commuting to work) for an extended period of time (e.g., one year). In order to collect such a dataset, the National Highway Traffic Safety Administration, the ITS Joint Program Office of the Federal Highway Administration, the Federal Motor Carrier Safety Administration, the Virginia Department of Transportation, and Virginia Tech contracted with the Virginia Tech Transportation Institute to conduct the "100-Car Naturalistic Driving Study.” This large-scale naturalistic driving study was conducted using 100 instrumented vehicles (80 privately-owned and 20 leased vehicles). This data collection effort was conducted in the Washington, DC metropolitan area on a variety of urban, suburban, and rural roadways over a span of 13 months.

This publication is considered a final report and does not supersede another publication.

\section{Notice}

This document is disseminated under the sponsorship of the Department of Transportation in the interest of information exchange. The United States Government assumes no liability for its contents or use thereof.

This report does not constitute a standard, specification, or regulation.

The United States Government does not endorse products or manufacturers. Trade or manufacturers' names appear herein only because they are considered essential to the object of this document. 
Technical Report Documentation Page (Form 1700.7)

\begin{tabular}{|c|c|c|c|c|}
\hline \multicolumn{3}{|l|}{$\begin{array}{l}\text { 1. Report No. } \\
\text { FMCSA-RRR-06-004 }\end{array}$} & \multicolumn{2}{|c|}{ 3. Recipient's Catalog No. } \\
\hline \multirow{2}{*}{\multicolumn{3}{|c|}{$\begin{array}{l}\text { 4. Title and Subtitle } \\
\text { The 100-Car Naturalistic Driving Study: A Descriptive Analysis of } \\
\text { Light Vehicle-Heavy Vehicle Interactions from the Light Vehicle } \\
\text { Driver's Perspective, Data Analysis Results }\end{array}$}} & \multicolumn{2}{|c|}{$\begin{array}{l}\text { 5. Report Date } \\
\text { March } 2006\end{array}$} \\
\hline & & & \multicolumn{2}{|c|}{ 6. Performing Organization Code } \\
\hline \multicolumn{3}{|c|}{$\begin{array}{l}\text { 7. Authors } \\
\text { Hanowski, R. J., Olson, R. L., Hickman, J. S., Dingus, T. A. }\end{array}$} & \multicolumn{2}{|c|}{ 8. Performing Organization Report No. } \\
\hline \multirow{2}{*}{\multicolumn{3}{|c|}{$\begin{array}{l}\text { 9. Performing Organization Name and Address } \\
\text { Virginia Tech Transportation Institute } \\
3500 \text { Transportation Research Plaza (0536) } \\
\text { Blacksburg, Virginia } 24061\end{array}$}} & \multicolumn{2}{|c|}{ 10. Work Unit No. (TRAIS) } \\
\hline & & & \multicolumn{2}{|c|}{$\begin{array}{l}\text { 11. Contract or Grant No. } \\
\text { DTNH22-00-C-07007 }\end{array}$} \\
\hline \multirow{2}{*}{\multicolumn{3}{|c|}{$\begin{array}{l}\text { 12. Sponsoring Agency Name and Address } \\
\text { National Highway Traffic Safety Admin. (NHTSA) } \\
\text { NPO-113, Room } 6220 \\
\text { Office of Advanced Safety Research } \\
400 \text { Seventh Street SW, Room } 6220 \\
\text { Washington, DC } 20590\end{array}$}} & \multicolumn{2}{|c|}{$\begin{array}{l}\text { 13. Type of Report and Period Covered } \\
\text { Final Report } \\
\text { August } 2001 \text { to July } 2005\end{array}$} \\
\hline & & & \multicolumn{2}{|c|}{$\begin{array}{l}\text { 14. Sponsoring Agency Code } \\
\text { NHTSA NPO-113 }\end{array}$} \\
\hline \multicolumn{5}{|c|}{$\begin{array}{l}\text { 15. Supplementary Notes } \\
\text { Robert J. Carroll was the FMCSA COTR for this project. }\end{array}$} \\
\hline \multicolumn{5}{|c|}{$\begin{array}{l}\text { 16. Abstract } \\
\text { There have been several studies that have investigated interactions between light and heavy vehicles. These have } \\
\text { primarily consisted of crash database analyses where Police Accident Reports have been studied. These } \\
\text { approaches are generally reliable, but they do have limitations. Hanowski, Keisler, and Wierwille (2004) } \\
\text { addressed these limitations using a naturalistic approach to investigate light vehicle-heavy vehicle (LV-HV) } \\
\text { interactions. In their study, HVs were instrumented with a variety of data collection equipment including video } \\
\text { cameras. However, one of the limitations in their study was the lack on instrumentation in LVs. }\end{array}$} \\
\hline \multicolumn{5}{|c|}{$\begin{array}{l}\text { These limitations were addressed in the 100-Car Study by installing video cameras and other data collection } \\
\text { equipment on LVs (Dingus et al., 2004). All identified LV-HV interactions from the 100-Car data set were } \\
\text { included in the current report. Data analysts reviewed each LV-HV interaction event and coded the Incident Type, } \\
\text { Primary Maneuver, Contributing Factor(s), Accident Type, and Critical Reason(s). This project's primary goals } \\
\text { were to: (1) gain a better understanding of LV-HV interactions, (2) continue to develop a classification scheme for } \\
\text { LV-HV interactions, (3) compare the current data to the data obtained in the Hanowski, Keisler, and Wierwille } \\
\text { (2004) study for a more complete picture of the LV-HV interaction problem, and (4) provide background } \\
\text { information that would serve as a necessary prerequisite to the development of countermeasures for LV-HV } \\
\text { interactions. }\end{array}$} \\
\hline \multicolumn{2}{|c|}{$\begin{array}{l}\text { 17. Key Words } \\
\text { 100-Car, Contributing Factor, Critical Incident, } \\
\text { Critical Reason, Distraction, Driver Behavior, } \\
\text { Interaction, Light Vehicle-Heavy Vehicle, Naturalistic }\end{array}$} & \multicolumn{3}{|c|}{$\begin{array}{l}\text { 18. Distribution Statement } \\
\text { This document is available to the public through the } \\
\text { National Technical Information Service, Springfield, VA } \\
22161\end{array}$} \\
\hline $\begin{array}{l}\text { 19. Security Classif. } \\
\text { (of this report) } \\
\text { Unclassified }\end{array}$ & $\begin{array}{l}\text { 20. Security Classif. } \\
\text { (of this page) } \\
\text { Unclassified }\end{array}$ & $\begin{array}{l}\text { 21. No. of } \\
184\end{array}$ & & 22. Price \\
\hline
\end{tabular}




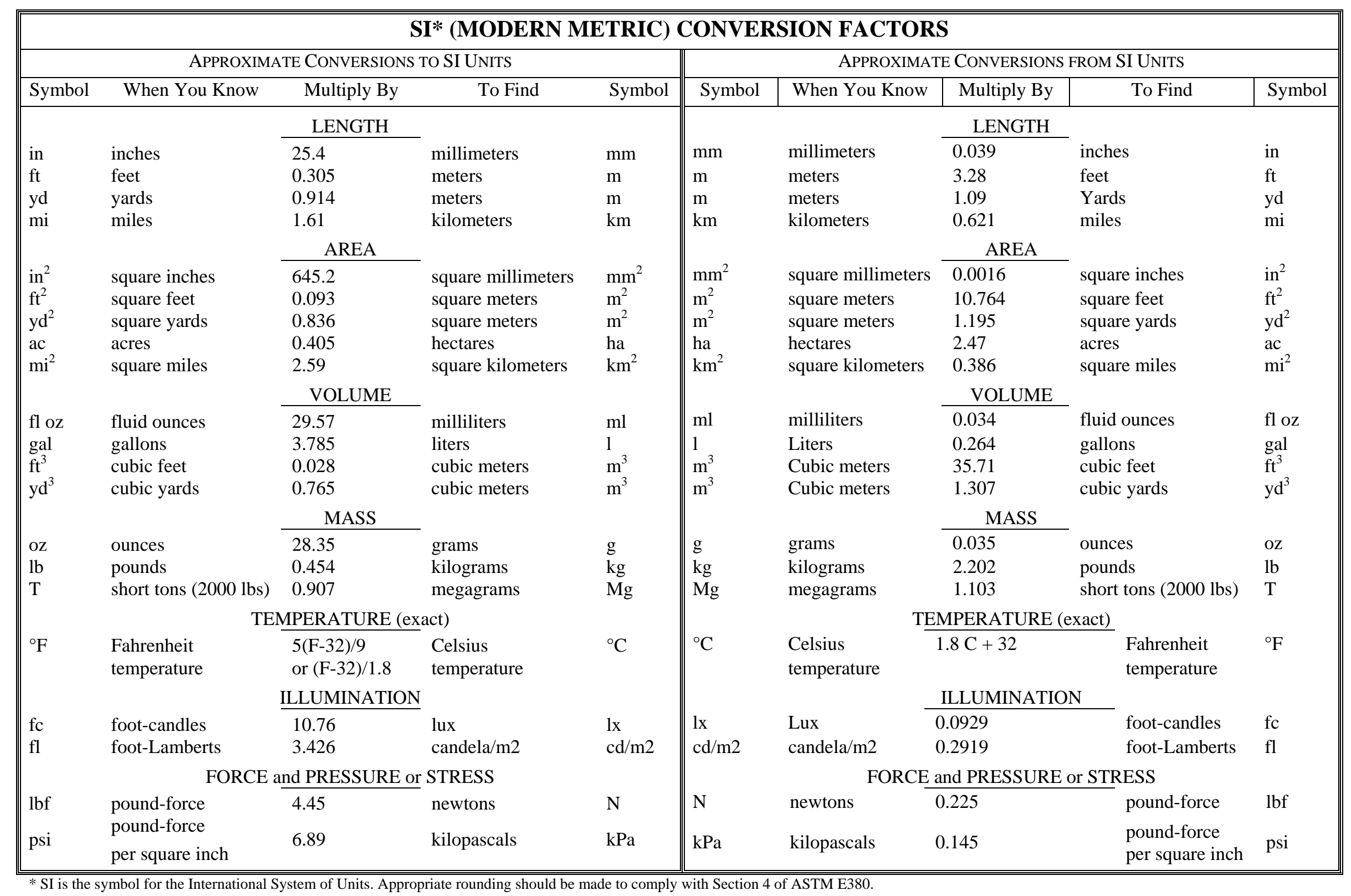

* SI is the symbol for the International System of Units. Appropriate rounding should be made to comply with Section 4 of ASTM E380. 


\section{ACKNOWLEDGEMENTS}

The research reported here was co-funded by the National Highway Traffic Safety Administration, the Federal Motor Carrier Safety Administration, and the Federal Highway Administration ITS Joint Program Office under Contract DTNH22-00-C-07007 (Task Order Number 06). The Task Order Manager was Jack Ference. The analysis presented in this report was requested by the Federal Motor Carrier Safety Administration. Mr. Robert Carroll was the FMCSA representative directing this analysis. Dr. Tom Dingus of the Virginia Tech Transportation Institute (VTTI) was the Principal Investigator on this project. The authors recognize Sheila Klauer and David Ramsey of VTTI for their assistance with this analysis effort. Thanks to Walter Wierwille for his guidance and to Robin Oakes of VTTI for her editorial support. Also, we wish to thank FMCSA's Richard Gruberg for his helpful comments on this report. 



\section{TABLE OF CONTENTS}

EXECUTIVE SUMMARY .

The Light Vehicle - Heavy Vehicle Interaction Problem.

.

Project Goals.......................

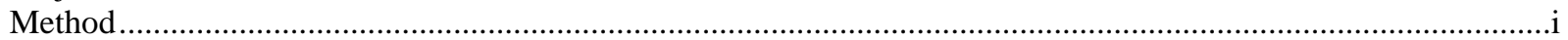

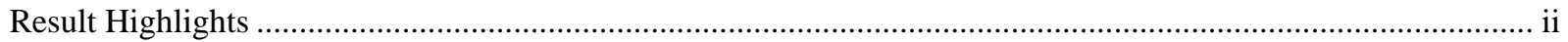

Light Vehicle-Heavy Vehicle Interaction Data Set ……........................................................................... ii

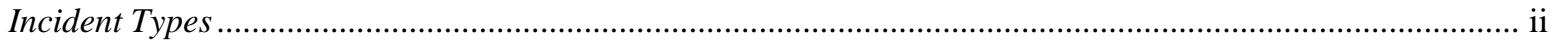

Primary Maneuvers, Secondary Maneuvers and Conflict Types ................................................................... iii

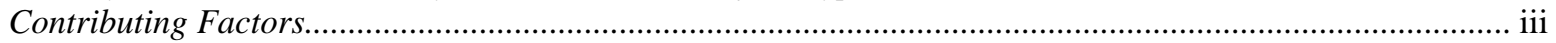

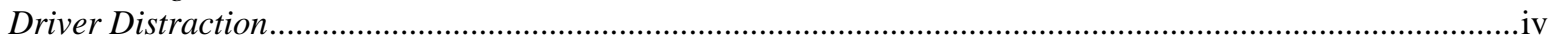

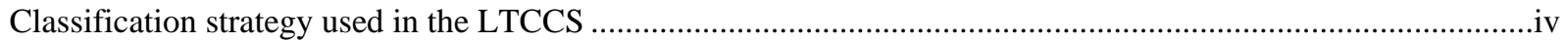

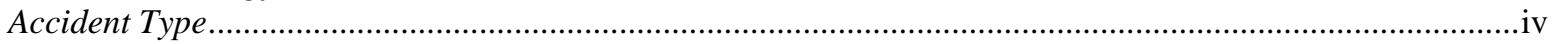

Critical Reason for the Critical Event................................................................................................................

Comparisons using the 100-Car Data, the Local Short Haul Data, and the Sleeper Berth Data ................................v

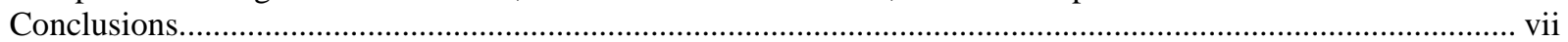

CHAPTER 1: LIGHT VEHICLE - HEAVY VEHICLE INTERACTIONS COLLECTED IN THE 100-CAR

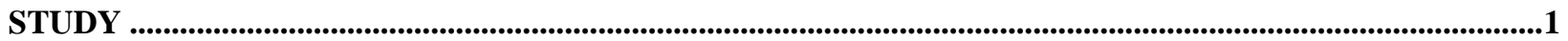

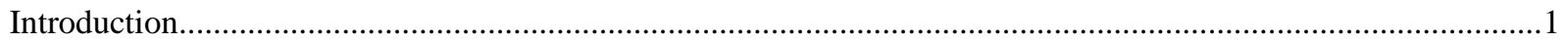

Overview of the Light Vehicle-Heavy Vehicle Problem ....................................................................................

Prior Research on Light Vehicle-Heavy Vehicle Interactions..................................................................................... 1

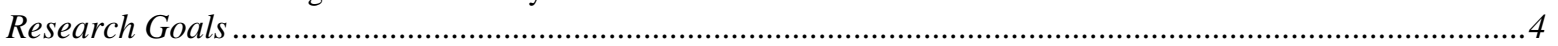

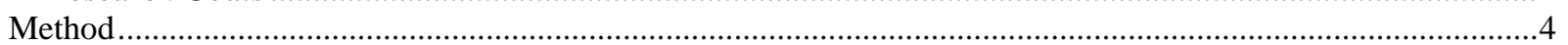

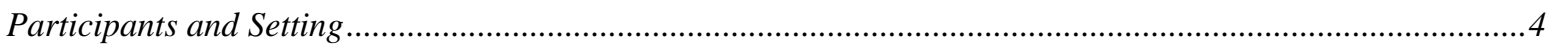

Light Vehicle Types...............................................................................................................................

Data Collection Methodology for the 100-Car Study ……...........................................................................6

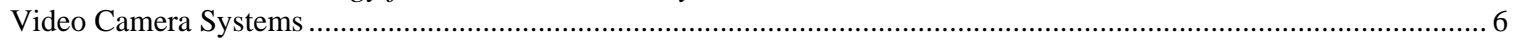

Video Recording Operation ...................................................................................................................... 8

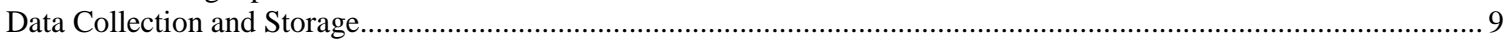

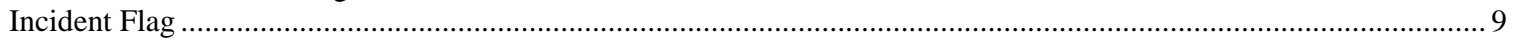

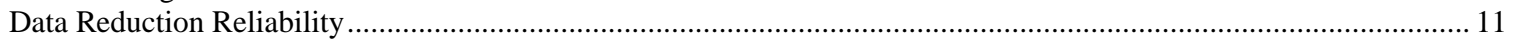

Strengths and Limitations of the Methodology Used .......................................................................................12

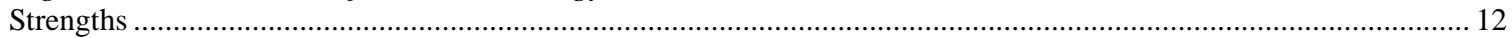

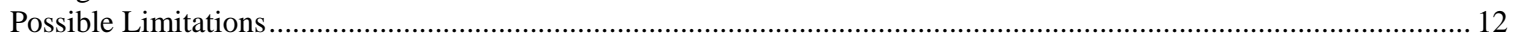

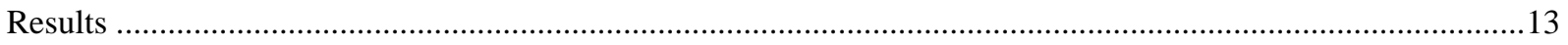

Light Vehicle-Heavy Vehicle Interaction Data Set ……...............................................................................13

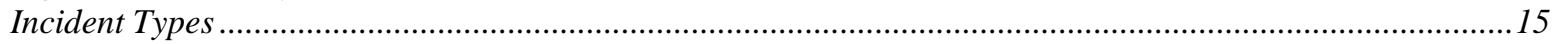

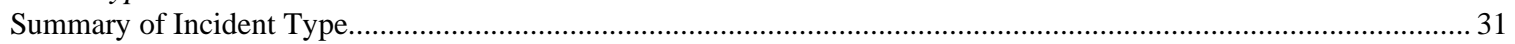

Primary Maneuvers, Secondary Maneuvers and Conflict Types ....................................................................31

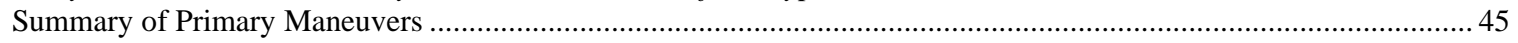

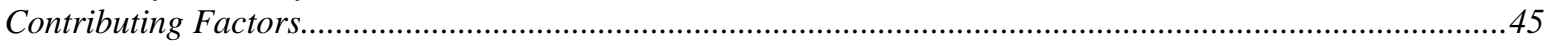

Contributing Factors X Incident Type Summary ………………………………………………………………. 55

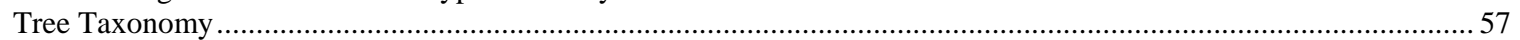

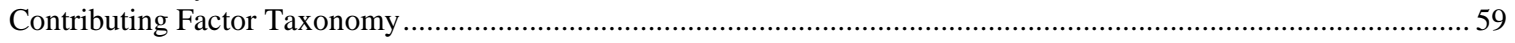

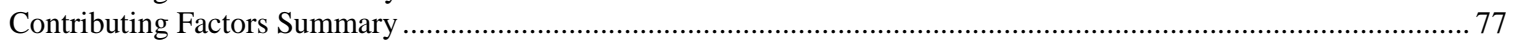

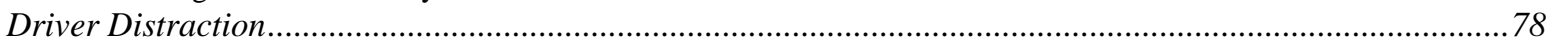

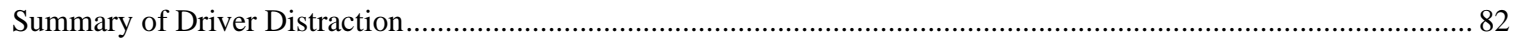

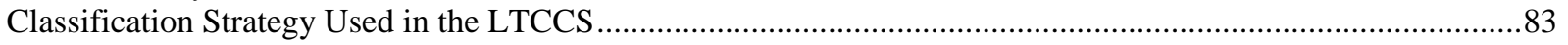

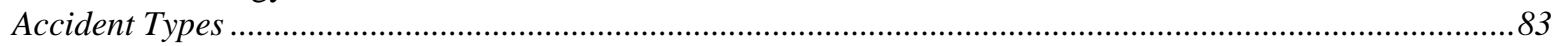

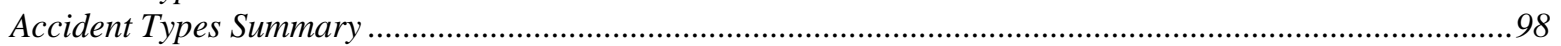

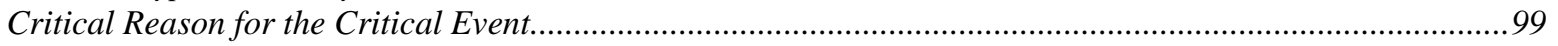

Critical Reasons X Incident Type Summary …………………………………………………………………... 102

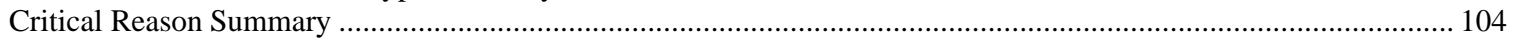

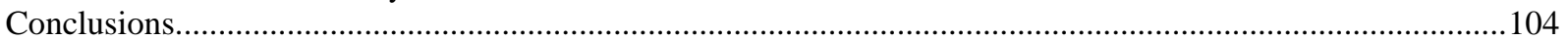


CHAPTER 2: COMPARISON OF THE RESULTS FROM THE ANALYSES CONDUCTED WITH THE 100-CAR DATA AND THE LOCAL/SHORT HAUL AND SLEEPER BERTH DATA......................................109

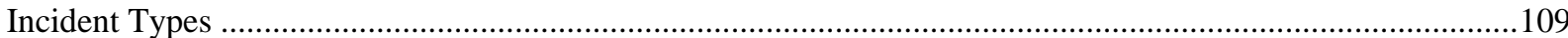

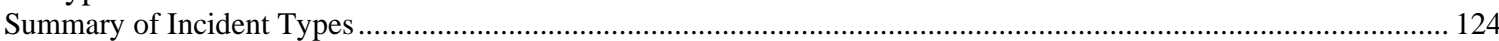

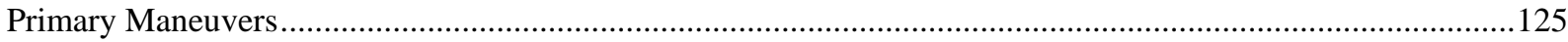

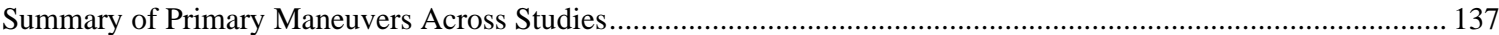

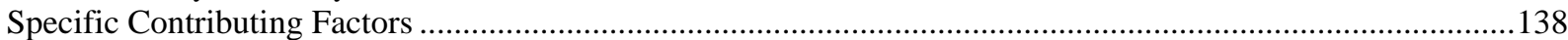

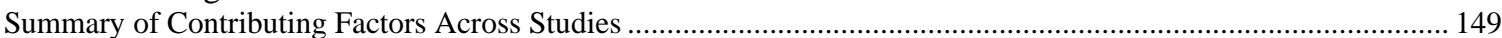

Discussion of the Results Comparing the 100-car, SB, and L/SH Studies ...................................................150

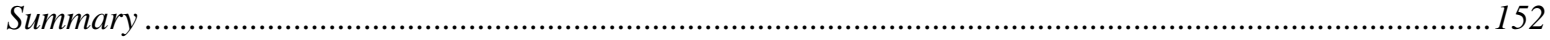

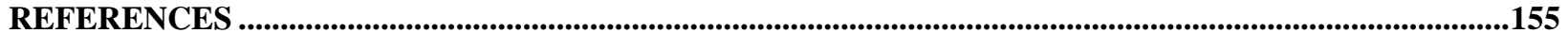




\section{LIST OF FIGURES}

FiguRE 1. TOYOTA CAMRY AND TOYOTA COROLLA USED IN 100-CAR STUDY. .........................................................6

FIGURE 2. FORD EXPLORER AND FORD TAURUS USED IN 100-CAR STUDY. .........................................................

FIGURE 3. THE FIVE CAMERA VIEWS RECORDED IN THE INSTRUMENTED VEHICLE. ..................................................

FIGURE 4. DIAGRAM OF THE MULTIPLEXED CAMERA VIEWS...........................................................................

FIGURE 5. SPLIT-SCREEN PRESENTATION OF THE FIVE CAMERA VIEWS....................................................................

FIGURE 6. DISTRIBUTION OF THE 9,125 INCIDENTS CAPTURED IN 100-CAR STUDY.................................................15

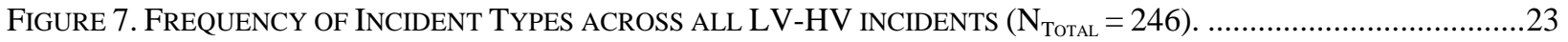

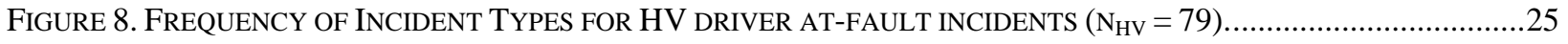

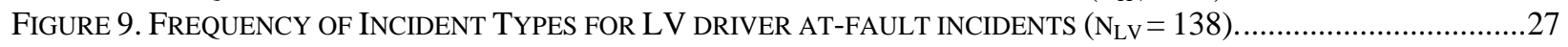

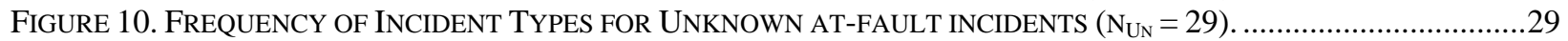

FIGURE 11. FREQUENCY OF INCIDENT TYPES FOR HV, LV, AND UNKNOWN AT-FAULT INCIDENTS $\left(\mathrm{N}_{\mathrm{HV}}=79, \mathrm{~N}_{\mathrm{LV}}=138\right.$,

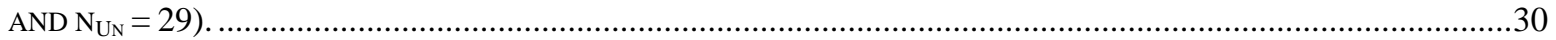

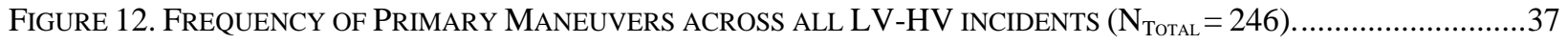

FigURE 13. FREQUENCY OF PRIMARY MANEUVERS FOR HV DRIVER AT-FAULT INCIDENTS $\left(\mathrm{N}_{\mathrm{HV}}=79\right) \ldots \ldots \ldots \ldots \ldots \ldots \ldots . . . . . . . . . .39$

FigURE 14. FREQUENCY OF PRIMARY MANEUVERS FOR LV DRIVER AT-FAULT INCIDENTS $\left(\mathrm{N}_{\mathrm{LV}}=138\right) \ldots \ldots \ldots \ldots . . . . . . . . . .41$

FiguRE 15. FREQUENCY OF PRIMARY MANEUVERS FOR UNKNOWN AT-FAULT INCIDENTS $\left(\mathrm{N}_{\mathrm{UN}_{\mathrm{N}}}=29\right)$........................43

FiguRE 16. FREQUENCY OF PRIMARY MANEUVERS FOR HV, LV, AND UNKNOWN AT-FAULT INCIDENTS $\left(\mathrm{N}_{\mathrm{HV}}=79\right.$, $\mathrm{N}_{\mathrm{LV}}$ $=138$, AND $_{\mathrm{UN}}=29$ ).

FIGURE 17. FREQUENCY OF CONTRIBUTING FACTORS ACROSS ALL LV-HV INCIDENTS $\left(\mathrm{N}_{\text {TOTAL }}=246.\right)$........................48

FIGURE 18. FREQUENCY OF CONTRIBUTING FACTORS FOR LV DRIVERS IN THE HV AT-FAULT INCIDENTS $\left(\mathrm{N}_{\mathrm{HV}}=79\right) .50$

FIGURE 19. FREQUENCY OF CONTRIBUTING FACTORS FOR LV DRIVER AT-FAULT INCIDENTS $\left(\mathrm{N}_{\mathrm{LV}}=138\right) \ldots \ldots \ldots \ldots \ldots \ldots . . . .52$

FIGURE 20. FREQUENCY OF CONTRIBUTING FACTORS FOR UNKNOWN AT-FAULT INCIDENTS $\left(\mathrm{N}_{\mathrm{UN}_{\mathrm{N}}}=29\right) \ldots \ldots \ldots \ldots \ldots \ldots . . . . .54$

FigURE 21. TAXONOMY OF THE CONTRIBUTING FACTORS FOR LV DRIVERS IN THE HV AT-FAULT INCIDENTS $\left(\mathrm{N}_{\mathrm{HV}}=\right.$ 79).

FIGURE 22. TAXONOMY OF THE CONTRIBUTING FACTORS FOR LV DRIVER AT-FAULT INCIDENTS $\quad\left(\mathrm{N}_{\mathrm{HV}}=138\right) . . . . .58$

FIGURE 23. TAXONOMY OF THE CONTRIBUTING FACTORS FOR UNKNOWN AT-FAULT INCIDENTS $\quad\left(\mathrm{N}_{\mathrm{UN}}=29\right)$........59

FIGURE 24. TAXONOMY STRUCTURE USED TO CHARACTERIZE THE HV DRIVER AT-FAULT INCIDENTS $\left(\mathrm{N}_{\mathrm{HV}}=79\right) \ldots . . . .60$

FIGURE 25. TAXONOMY STRUCTURE USED TO CHARACTERIZE THE LV DRIVER AT-FAULT INCIDENTS $\left(\mathrm{N}_{\mathrm{LV}}=138\right) \ldots . . .66$

FIGURE 26. TAXONOMY STRUCTURE USED TO CHARACTERIZE THE UNKNOWN AT-FAULT INCIDENTS $\left(\mathrm{N}_{\mathrm{UN}_{\mathrm{N}}}=29\right) \ldots . . . . .74$

FIGURE 27. FREQUENCY OF THE DISTRACTION CONTRIBUTING FACTOR IN EACH SUB-CATEGORY................................79

FIGURE 28. FREQUENCY OF ACCIDENT TYPES ACROSS THE ENTIRE DATA SET $\left(\mathrm{N}_{\text {TOTAL }}=246\right)$.....................................87

FIGURE 29. FREQUENCY OF ACCIDENT TYPES FOR HV DRIVER AT-FAULT INCIDENTS $\left(\mathrm{N}_{\mathrm{HV}}=79\right)$............................90

FIGURE 30. FREQUENCY OF ACCIDENT TYPES FOR LV DRIVER AT-FAULT INCIDENTS $\left(\mathrm{N}_{\mathrm{LV}}=138\right)$............................93

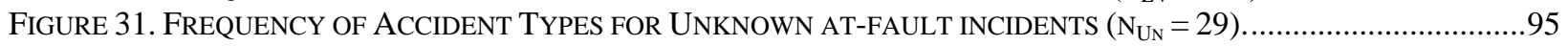

FiguRE 32. FREQUENCY OF ACCIDENT TYPES FOR HV, LV, AND UNKNOWN AT-FAULT INCIDENTS ( $\mathrm{N}_{\mathrm{HV}}=79, \mathrm{~N}_{\mathrm{LV}}=$

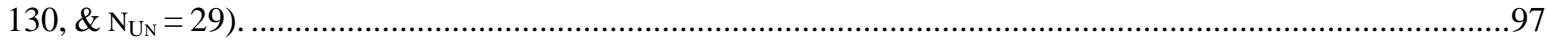

FIGURE 33. FREQUENCY OF CRITICAL REASONS FOR LV DRIVER AT-FAULT INCIDENTS ( $\left.\mathrm{N}_{\mathrm{LV}}=138\right) \ldots \ldots . . . . . . . . . . . . . . . . .101$

FIGURE 34. PERCENTAGE OF INCIDENT TYPES ACROSS ALL THREE STUDIES............................................................111

FiguRE 35. PERCENTAGE OF INCIDENT TYPES IN THE 100-CAR, SB, L/SH STUDIES. .............................................117

FiguRE 36. PERCENTAGE OF HV DRIVER AT-FAULT INCIDENT TYPES IN THE 100-CAR, SB, AND L/SH STUDIES. .......119

FiguRE 37. PERCENTAGE OF LV DRIVER AT-FAULT INCIDENT TYPES IN THE 100-CAR, SB, AND L/SH STUDIES.........121

FigURE 38. PERCENT OF INCIDENTS IN THE 100-CAR, SB, AND L/SH STUDIES AS A FUNCTION OF ROAD TYPE...........123

FIGURE 39. FREQUENCY OF PRIMARY MANEUVERS ACROSS THE THREE STUDIES. ...................................................127

FiguRE 40. PERCENTAGE OF PRIMARY MANEUVERS IN THE 100-CAR, SB, AND L/SH STUDIES.................................132

Figure 41. PERCENTAGE OF HV DRIVER AT-FAULT INCIDENTS IN THE 100-CAR, SB, AND L/SH STUDIES AS A

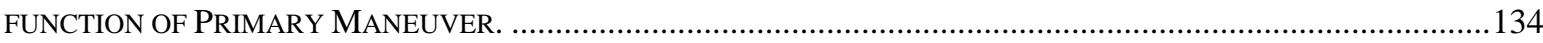

Figure 42. PERCENTAGE OF LV DRIVER AT-FAUlt INCIDENTS IN THE 100-CAR, SB, AND L/SH STUDIES AS A

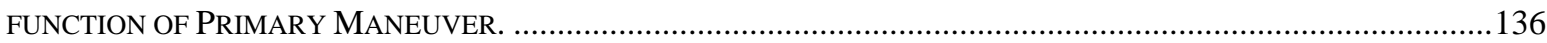

FIGURE 43. FREQUENCY OF CONTRIBUTING FACTORS ACROSS THE 100-CAR, SB, AND L/SH STUDIES.......................139

FigURE 44. PERCENTAGE OF INCIDENTS IN THE 100-CAR, SB, AND L/SH STUDIES AS A FUNCTION OF CONTRIBUTING

FACTORS. 144 
Figure 45. PERCENTAge Of HV at-FAUlt INCIDENTS IN THE 100-CAR, SB, AND L/SH STUDiES AS A FUNCTION OF

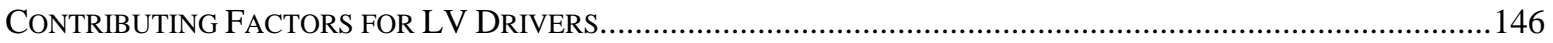
Figure 46. PERCENTAGE OF LV DRIVER AT-FAULT INCIDENTS IN THE 100-CAR, SB, AND L/SH STUDIES AS A FUNCTION OF CONTRIBUTING FACTOR... 


\section{LIST OF TABLES}

TABle 1. Participant Age and Gender Distributions.

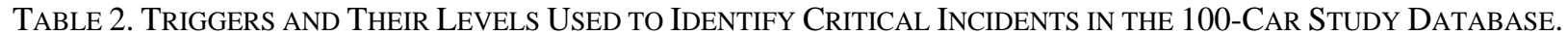

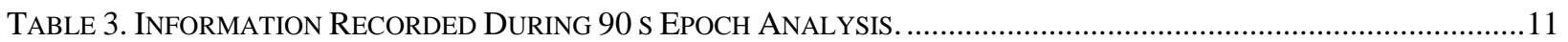

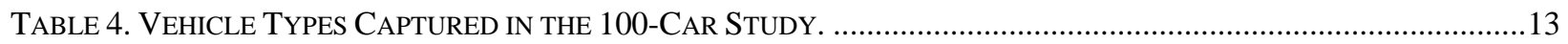

TABLE 5. DESCRIPTION OF THE INCIDENT TYPES THAT WERE IDENTIFIED IN THE CURRENT RESEARCH........................16

TABle 6. Frequency, Percentage, and RANK ORDERING of THE INCIDENT TyPES ACROSS ALL LV-HV INCIDENTS

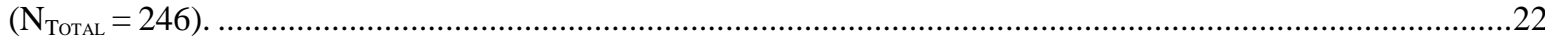

TABle 7. FRequency, PeRCENTAGE, AND RANK ORDERING OF THE INCIDENT TYPES FOR HV DRIVER AT-FAULT

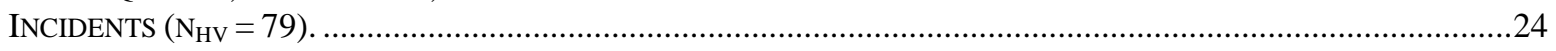

Table 8. Frequency, Percentage, and RANK ORdering of the InCIDENT TyPeS For LV DRIVER At-Fault

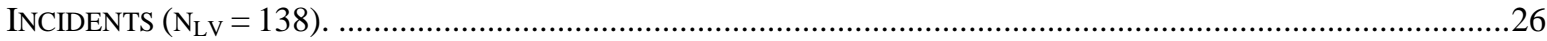

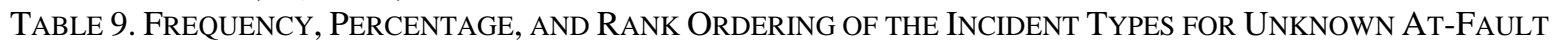

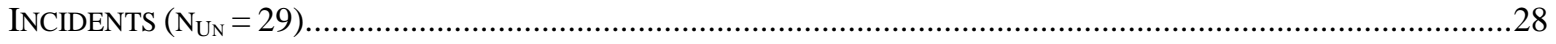

TABLE 10. LIST AND DEFINITION OF EACH PRIMARY MANEUVER TYPES.................................................................31

TABLE 11. THE 66 DifFerent Conflict TyPes IDENTIFIED ACROSS ALL LV-HV INCIDENTS.......................................33

TABle 12. Frequency, Percentage, and RANK ORdering of the Primary MANEuvers ACRoss ALl LV-HV

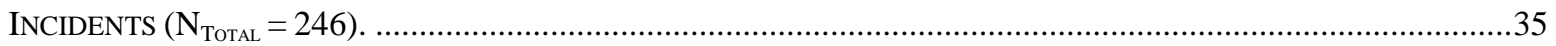

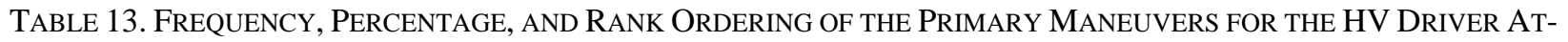

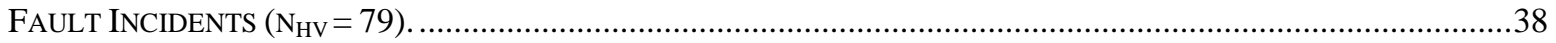

TABle 14. Frequency, Percentage, And RAnK Ordering of the Primary Maneuvers for the LV Driver At-

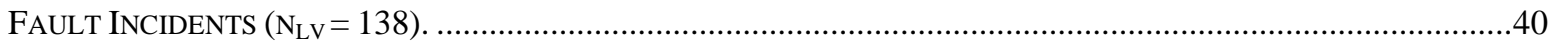

Table 15. Frequency, Percentage, and Rank Ordering of the Primary Maneuvers For the UnKNown At-

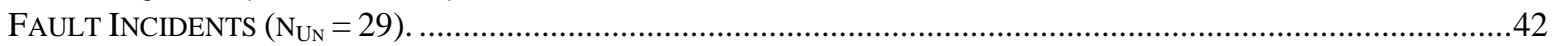

TABLE 16. CONTRIBUTING FACTORS USED TO IDENTIFY THE PRIMARY CAUSE OF THE INCIDENT. ...............................46

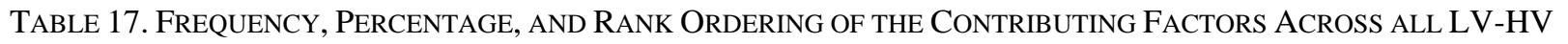

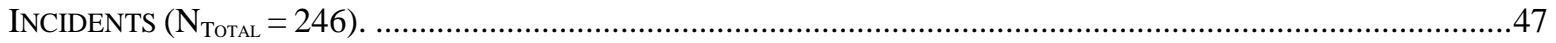

TABle 18. Frequency, Percentage, AND RANK ORdering Of the Contributing Factors For HV DRIVERS IN

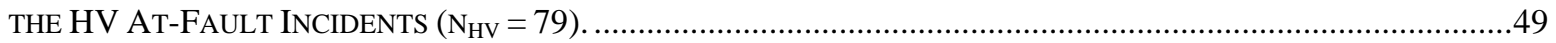

Table 19. Frequency, Percentage, and Rank Ordering of the Contributing Factors For the LV DRIVER

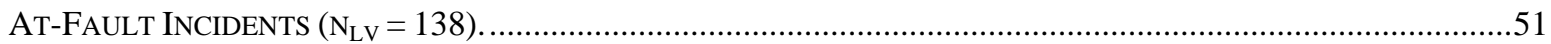

Table 20. Frequency, Percentage, and Rank Ordering of the Contributing Factors For the UnKNown

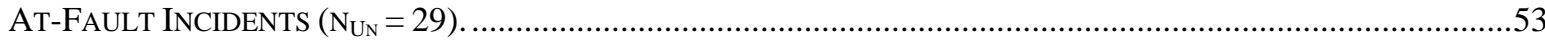

TABLE 21. FREQUENCY OF INCIDENT TYPES X CONTRIBUTING FACTORS ACROSS LV DRIVER AT-FAULT INCIDENTS

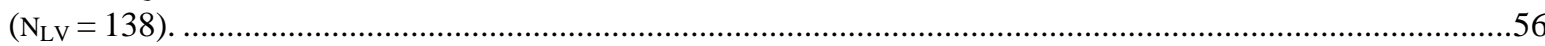

TABle 22. Frequency, Percentage, and Rank ORDERING OF EACH Sub-CATEgory in the Distraction

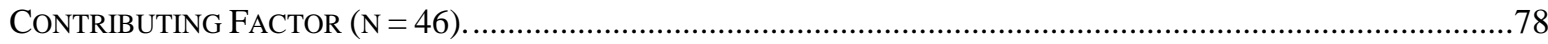

TABle 23. Frequency, Percentage, and Rank ORdering of EACH Sub-Category in the Distraction CONTRIBUTING FACTOR FOR THE HV AT-FAULT INCIDENTS ( $=9$ ) ...............................................................8

Table 24. Frequency, Percentage, and Rank Ordering of Each Sub-Category in the Distraction

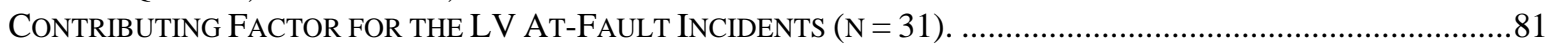

Table 25. Frequency, Percentage, and Rank ORdering of Each Sub-Category in the Distraction

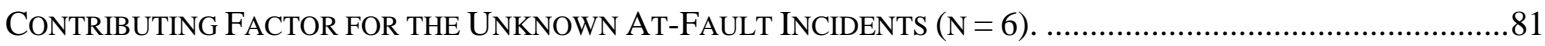

TABLE 26. DESCRIPTION OF THE LTCCS ACCIDENT TYPES (THIERIEZ, RADJA, AND TOTH, 2002)..............................84

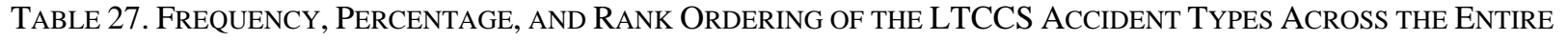

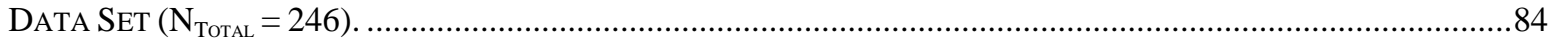

TABle 28. Frequency, PERCENTAGe, AND RANK ORDERING OF THE ACCIDENT TyPES FOR HV DRIVER AT-FAult INCIDENTS $\left(\mathrm{N}_{\mathrm{HV}}=79\right)$. 
Table 29. Frequency, Percentage, and RANK Ordering of the Accident Types For LV Driver At-Fault

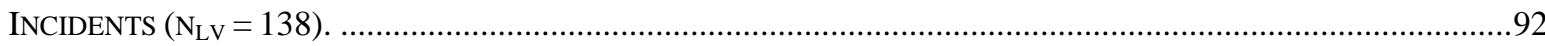

TABle 30. Frequency, PerCentage, AND RANK ORdERING OF THE ACCIDENT TyPES WHERE IT WAS UNKNOWN IF THE LV OR THE HV DRIVER WAS AT-FAULT ( $\left.\mathrm{N}_{\mathrm{UN}}=29\right)$

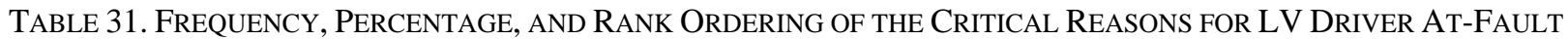
INCIDENTS $\left(\mathrm{N}_{\mathrm{LV}}=138\right)$. 100

TABle 32. FREQUENCY of INCIDENT TyPES X CRITICAl REASONS FOR LV DRIVER AT-FAUlt INCIDENTS $\left(\mathrm{N}_{\mathrm{LV}}=138\right)$.

TABLE 33. COMPARISON OF A SELECTION OF RESUlts IN THE CURRENT STUDY WITH PRIOR LV-HV INTERACTION STUDIES 107

TABLE 34. FREQUENCY, PERCENTAGE, AND RANK ORDERING OF THE INCIDENT TYPES ...........................................110

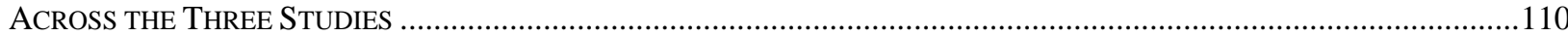

TABLE 35. FREQUENCY AND PERCENTAGE OF INCIDENT TyPES FOR THE 100-CAR STUDY.......................................113

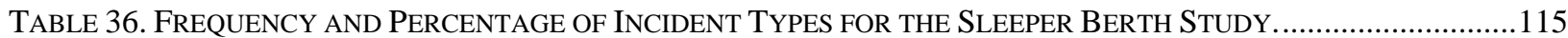

TABLE 37. FREQUENCY AND PERCENTAGE OF INCIDENT TYPES FOR THE LOCAL/SHORT HAUL STUDY.......................116

TABLE 38. FREQUENCY, PERCENTAGE, AND RANK ORDERING OF PRIMARY MANEUVERS ACROSS THE THREE STUDIES.

TABLE 39. FREQUENCY AND PERCENTAGE OF PRIMARY MANEUVERS FOR THE 100-CAR STU

TABle 40. Frequency and Percentage of Primary MANeuvers For the SLeEPER Berth STUdy.....................130

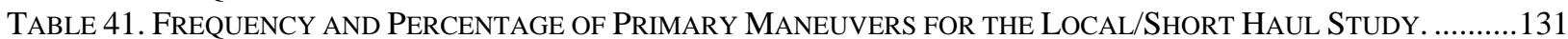

TABle 42. FreQuency, PERCENTAge, AND RANK ORDERING OF THE CONTRIBUting FACTORS ACROSS THE 100-CAR,

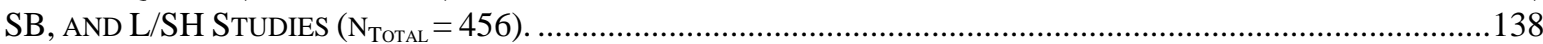

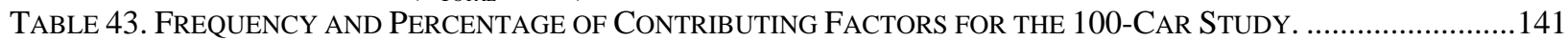

TABLE 44. Frequency AND PERCENTAGe Of Contributing FACTORS FOR THE SLEEPER BERTH STUDY................141

TAble 45. Frequency and Percentage of Contributing Factors For the LoCal/Short Haul Study. .......143 


\section{EXECUTIVE SUMMARY THE LIGHT VEHICLE - HEAVY VEHICLE INTERACTION PROBLEM}

In 2002, 434,000 large trucks (gross weight $\geq 10,000 \mathrm{lbs}$ ) were involved in vehicle crashes; 4,542 of these crashes resulted in fatalities. In these crashes, 4,897 people died and an additional 210,000 were injured. Though accounting for $4 \%$ of all registered vehicles in 2002, large trucks represented 8\% of all vehicles involved in fatal crashes (National Highway Traffic Safety Administration, NHTSA, 2003). However, truck drivers have lower non-fatal crash rates per million vehicle miles traveled than light vehicles (NHTSA, 2003). Nonetheless, light vehicles are extremely vulnerable when they interact with trucks because trucks often weigh 20-30 times as much as light vehicles (Insurance Institute for Highway Safety, 2002), and trucks take 20-40\% farther to stop than light vehicles (NHTSA, 1987). A better understanding of LV-HV interactions is needed to develop future interventions and countermeasures directed at mitigating the problem. The data from the 100-Car Study (Dingus et al., 2004) were used in the current project to assess the LV-HV interaction problem from the LV drivers’ perspective.

\section{PROJECT GOALS}

There were four primary goals in the current effort:

1. Gain a better understanding of LV-HV interactions on the nation's roadways.

2. Continue to develop the classification scheme and corresponding Contributing Factors list for LV-HV interactions used in Hanowski, Keisler, and Wierwille (2004) and use the terminology and methodology described in the LTCCS (Thieriez, Radja, and Toth, 2002).

3. Compare the current data to the data obtained in the Hanowski, Keisler, and Wierwille (2004) study for a more complete picture of the LV-HV interaction problem.

4. Provide background information that would serve as a necessary prerequisite to the development of countermeasures for LV-HV interactions.

\section{METHOD}

One hundred and nine participants who commute to and from the Washington, DC metro area were recruited as drivers in the 100-Car Study. The 109 participants ranged in age from 18 to over 55 years (43 female, 66 male). One hundred LVs were instrumented for this study; 80 vehicles were owned by the participants, while 20 were leased vehicles from VTTI.

The data used in the current effort consisted of video recordings of critical incidents. Five video cameras were used in the video recording system: (1) a forward-looking camera that captured the forward roadway scene, traffic situation, and possible incidents; (2) a driver's face camera that was used to record facial expressions, eyelid closure, glance position, and head turns; (3) a rightside camera that was mounted on the A-pillar of the passenger side and faced outward; (4) a dome camera that was mounted from inside the vehicle and faced over the drivers shoulder towards the steering wheel, hands, and feet; and (5) a rear camera that was intended to capture the situation behind the vehicle. Infrared lighting was used to illuminate the vehicle cab so that the driver's face as well as their hands could still be viewed by the camera during nighttime driving. 
The video continuously recorded while the ignition was on, thereby allowing laboratory review and selection of the video without losses of any kind. The videotaped episodes/incidents were selected and keyed to digitally-recorded data.

Of interest in the data set were "critical incidents" defined as unexpected events resulting in a close call or requiring fast action (evasive maneuver) on the part of a driver to avoid a crash. Critical incidents were detected by one of three methods. The first method involved flagging events where the car sensors exceeded a specified value (e.g., brake response of $>0.6 \mathrm{~g}$ ). The second incident flagging method occurred when the driver pressed an incident pushbutton located on the dashboard (i.e., drivers were instructed to depress a button on the dashboard if they witnessed an incident or were involved in an incident). The third method of detecting incidents was through analysts' judgments when reviewing the video. Only those events that involved a LV-HV interaction are described in the current analyses.

\section{RESULT HIGHLIGHTS}

\section{Light Vehicle-Heavy Vehicle Interaction Data Set}

The 100-Car Study captured 9,125 incidents, which were divided into four categories: (1) LVLV Interactions; (2) LV-HV Interactions; (3) Single Vehicle Conflicts; and (4) Other. Of the 9,125 events, 246 (2.7\%) involved a LV-HV interaction.

\section{Incident Types}

With the $246 \mathrm{LV}-\mathrm{HV}$ interactions recorded in the data set, the next step in the analysis was to determine the vehicles' actions for each incident. To this end, the video and relevant data for each incident were carefully reviewed and then classified according to "Incident Type.” Twentyseven different Incident Types were identified in the data set.

The most frequent Incident Type involving a LV-HV interaction was Late Braking for Stopped/Stopping Traffic. Across all 246 incidents, this particular Incident Type occurred 66 times and accounted for $26.8 \%$ of the incidents captured. The majority of the incidents (48.8\%) involved one of two different Incident Types: Late Braking for Stopped/Stopping Traffic, and Lane Change Without Sufficient Gap.

Descriptive statistics for the Incident Types were also calculated for incidents as a function of the at-fault driver. The at-fault driver is the driver that was assessed, by the analyst, to have been responsible for causing the event. Of the $246 \mathrm{LV}-\mathrm{HV}$ interaction incidents recorded, 138 (56\%) were judged to have been the fault of the LV driver, while 79 (32\%) were attributed to the HV driver. For the remaining 29 incidents (12\%), it was unclear which vehicle driver was at-fault. By removing the "unknown" cases from the LV-HV driver at-fault analyses, it was found that the LV driver was at-fault in 64\% (138/217) of the LV-HV interaction incidents, while the HV driver was at fault in $36 \%$ (79/217) of the incidents. 
The most frequent Incident Type for HV driver at-fault incidents was Lane Change Without Sufficient Gap (26.6\%), followed by Lateral Deviation of Through Vehicle (21.5\%), and Left Turn Without Clearance (13.9\%). The most frequent Incident Type for LV driver at-fault incidents was Late Braking for Stopped/Stopping Traffic (41.3\%), followed by Lane Change Without Sufficient Gap (21.7\%), and Aborted Lane Change (8\%). The most frequent Incident Type for Unknown at-fault incidents was Late Braking for Stopped/Stopping Traffic (27.6\%), followed by Conflict With Oncoming Traffic (13.8\%), Lane Change Without Sufficient Gap (10.3\%), and Unable to Determine (10.3\%).

\section{Primary Maneuvers, Secondary Maneuvers, and Conflict Types}

After each of the 246 incidents was classified by Incident Type, the next step in the analysis was to identify the "Primary Maneuvers" and "Secondary Maneuvers" involved in each incident. The Primary Maneuver refers to the maneuver of the driver who initiated the incident (not necessarily at-fault). Across the 246 interaction incidents, 19 different Primary Maneuvers were identified. The most frequent Primary Maneuvers were Braking (22.7\%), Changing Lanes (21.1\%), and Stopped (15\%). These three Primary Maneuvers represented 58.9\% of the recorded incidents.

In addition to identifying the Primary Maneuver for each incident, the Secondary Maneuver, or the maneuver of the responding driver (i.e., driver of the second vehicle involved in the interaction), was also classified. Considering the maneuvers of both vehicles involved in the incident, a clear picture of the conflict, or Conflict Type, could be determined. A total of 66 different Conflict Types (i.e., Primary Maneuver and Secondary Maneuver combinations) were identified.

\section{Contributing Factors}

Just as the Incident Types describe the action or what happened during an incident, Contributing Factors provide likely reasons why an incident occurred. For each incident that was analyzed, a number of Contributing Factors were identified. It should be noted that the Contributing Factor categories were taken from Wierwille, Kieliszewski, Hanowski, Keisler, and Olsen (2000) and from the GES Physical Impairments screen (USDOT/NHTSA, 2003, p. 434). Due to the methodology used, where the data collection equipment was only instrumented in the LV, the Contributing Factor was based solely on the behaviors of the LV driver. Without cameras inside the HV there was no way to determine, with any degree of certainty, the behavior(s) of the HV driver. Even when the HV driver was judged to have been at-fault, the behaviors of the LV driver were identified. Put another way, for the events that were caused by the LV driver, the analyses considered the LV driver's behaviors that may have contributed to the event. For events where the HV-driver was at-fault, the analysis also considered the LV driver's behaviors. However, the consideration is for the LV driver behaviors that occurred as the driver reacted to the HV driver's actions.

Note that multiple factors could be assessed for each individual event (as such, the percentages for the factors total more than 100\%). Across all 246 incidents, the most frequent Contributing Factor was Driving Techniques (49.5\%), followed by Unknown (24\%) and Distracted (18.7\%). 
The most frequent Contributing Factor for HV driver at-fault incidents was Unknown (68.4\%), followed by Driving Techniques (15.2\%), and Distracted (11.4\%). The most frequent Contributing Factor for LV driver at-fault incidents was Driving Techniques (70.3\%), followed by Distracted (22.5\%) and Aggressive Driving (22.5\%). The most frequent Contributing Factor for Unknown driver at-fault incidents was Driving Techniques (48.3\%), followed by Distracted (20.7\%), Roadway Alignment (10.3\%), and Unknown (10.3\%).

\section{Driver Distraction}

A substantial number of the LV-HV incidents had Distraction listed as a Contributing Factor. Again, as indicated above, the incidents where Driver Distraction was mentioned refer to the behavior of the LV driver. The Distraction Contributing Factor was sub-divided into more distinct categories. See Table 22 for a listing of the frequency, percentage, and rank ordering for each sub-category in the Distraction Contributing Factor. The most frequent sub-category for the Distraction Contributing Factor was Talking/Listening on Cell Phone (21.7\%), followed by Passenger in Adjacent Seat (13\%), and Dialing Hand-Held Phone (8.7\%).

\section{CLASSIFICATION STRATEGY USED IN THE LTCCS}

\section{Accident Type}

Each of the $246 \mathrm{LV}-\mathrm{HV}$ interactions were grouped by Accident Type based on the methodology used in the LTCCS (Thieriez, Radja, and Toth, 2002). Note that there was only one LV-HV crash recorded in the 100-Car Study. Therefore, using the Accident Types from the LTCCS does not reflect an absolute match, but rather a relative match. However, to facilitate future data comparisons with the near-crash data collected in the current study with other studies using the LTCCS, each of the $246 \mathrm{LV}-\mathrm{HV}$ interactions were coded using the LTCCS classification scheme. Because only one crash occurred, the closest match with respect to Accident Types was recorded for each incident.

Overall, the most frequent Accident Types were Scenarios 38/391: Same Trafficway/Same Direction: Forward Impact: Avoid Collision with Vehicle (19.9\%); 20/21: Same Trafficway/Same Direction: Rear-End: Approaches Stopped Vehicle (16.3\%); and 28/29: Same Trafficway/Same Direction: Rear-End: Approaches Decelerating Vehicle (13\%). These three Accident Types represented $49.2 \%$ of the Accident Types for all LV-HV incidents.

The Accident Types for HV and LV driver at-fault incidents differed. The most prevalent Accident Types for HV driver at-fault incidents were Scenarios 44/45: Same Trafficway/Same Direction: Sideswipe Angle: In Blind Spot (27.8\%), 38/39: Same Trafficway/Same Direction: Forward Impact: Avoid Collision with Vehicle (15.2\%), and 25/25: Same Trafficway/Same Direction: Rear-End: Approaches Slower Constant Speed Vehicle (8.9\%). These three Accident Types accounted for $51.9 \%$ of the HV driver at-fault incidents. The most prevalent Accident Types for LV driver at-fault incidents were Scenarios 20/21: Same Trafficway/Same Direction: Rear-End: Approaches Stopped Vehicle (26.8\%); 38/39: Same Trafficway/Same Direction: Forward Impact: Avoid Collision with Vehicle (22.5\%); and 28/29: Same Trafficway/Same

\footnotetext{
${ }^{1}$ Scenario classification taken from Thieriez, Radja, \& Toth, 2002.
} 
Direction: Rear-End: Approaches Decelerating Vehicle (17.4\%). These three Accident Types accounted for $66.7 \%$ at the $\mathrm{LV}$ driver at-fault incidents.

The most prevalent Accident Type for HV driver at-fault incidents involved a Sideswipe Angle. By summing all the HV driver at-fault Accident Types that involved a Sideswipe Angle, it was found that $41.8 \%$ of the HV driver at-fault incidents were coded with this Accident Type. Conversely, the most prevalent Accident Type for LV driver at-fault incidents involved a RearEnd Approach. By summing all LV driver at-fault Accident Types that involved a Rear-End approach, it was found that $55.1 \%$ of the LV driver at-fault incidents were coded with this Accident Type.

\section{Critical Reason for the Critical Event}

To be consistent with the LTCCS (Thieriez, Radja, and Toth, 2002), the LV driver at-fault incidents were coded with a Critical Reason for the incident. The Critical Reason for the incident was considered the primary reason for why the incident occurred. More than one Critical Reason could be coded for each incident (10 of the recorded incidents were coded with two Critical Reasons). Only the LV driver at-fault incidents were coded with a Critical Reason because those vehicles were equipped with video of the driver. For the HV driver at-fault incidents, it was not possible to determine with any certainty what the driver was doing that contributed to the event; therefore, all HV driver at-fault incidents were coded as "Unknown reason for the critical event."

Overall, the most frequent Critical Reasons for LV driver at-fault incidents were Aggressive Driving Behavior (24.6\%), Too Fast for Conditions (15.2\%), and Misjudgment of Gap (13.8\%). There were other interesting trends. Sixty-four of the $138 \mathrm{LV}$ at-fault incidents (46.4\%) were coded with at least one Critical Reason that was a risky driving behavior (i.e., Aggressive Driving Behavior, Too Fast for Conditions, Following too Closely, and Illegal Maneuver), while $22.5 \%$ of the LV driver at-fault incidents involved some type of awareness variable (i.e., Internal Distraction, Inattention, External Distraction).

\section{COMPARISONS USING THE 100-CAR DATA, THE LOCAL SHORT HAUL DATA, AND THE SLEEPER BERTH DATA}

The current study builds on a previous project that classified critical incidents (crashes and nearcrashes) recorded in two fatigue study with Local/Short Haul (L/SH) drivers and Sleeper Berth (SB) drivers (Hanowski, Keisler, and Wierwille, 2004). The two truck studies involved instrumentation in the truck and recorded events from the HV driver's perspective. In contrast, the current study recorded events as they unfolded from the LV driver's perspective. The events from the three studies were combined and the classifications were compared.

A total of $142 \mathrm{LV}-\mathrm{HV}$ interactions were identified in the L/SH study. Of these, 117 (82.4\%) incidents were judged to be the fault of the LV driver, while the remaining 25 (17.6\%) incidents were the fault of the HV driver. In the SB study, a total of $68 \mathrm{LV}-\mathrm{HV}$ interactions were 
identified. Of these, 47 (69.1\%) were assessed to have been the fault of the LV driver, while the remaining 21 (38.9\%) were the fault of the HV driver. Of the $246 \mathrm{LV}-\mathrm{HV}$ interaction incidents recorded in the current study, 138 (56\%) were judged to have been the fault of the LV driver, while 79 (32\%) were attributed to the HV driver. For the remaining 29 incidents (12\%), it was unclear which vehicle driver was at-fault. Considering the combined data, these three studies consistently show that LV drivers were judged to be responsible for the majority of LV-HV interactions. Of the $427 \mathrm{LV}-\mathrm{HV}$ incidents identified across the three studies (excluding the 29 Unknown at-fault incidents in the current study), 302 (70.7\%) were judged to have been the fault of the LV driver, while the remaining 125 (29.3\%) were the fault of the HV driver (a 2.4:1 ratio).

There were a number of interesting findings from the comparisons between the 100-Car, SB, and $\mathrm{L} / \mathrm{SH}$ studies. Comparisons were conducted with respect to the Incident Type, Primary Maneuver, and Contributing Factor. The Incident Type comparison indicated that Lane Change Without Sufficient Gap was the most frequent Incident Type across all three studies. A breakdown of incidents as a function of the at-fault driver showed that Lane Change Without Sufficient Gap incidents were primarily attributed to LV drivers. Critical incidents that involved a LV driver changing lanes in front of an HV, leaving the $\mathrm{HV}$ driver with very little headway between vehicles, were a common Incident Type that was captured in all three studies.

While the Incident Types for the LV driver at-fault incidents shared some similarities across the three studies, the Incident Types for the HV driver at-fault incident were more varied across the studies. In the 100-Car Study, $48.1 \%$ of the HV driver at-fault Incident Types included Lane Change Without Sufficient Gap or Lateral Deviation of Through Traffic. In the SB study, 71.4\% of the HV driver at-fault incidents included Late Braking for Stopped/Stopping Traffic and Following Too Closely. In the L/SH study, $48 \%$ of $\mathrm{HV}$ at-fault incidents included Roadway Entrance Without Clearance, Wide Turn Into Adjacent Lane, or Late Braking for Stopped/Stopping Traffic.

One possible explanation for these differences was the predominant Road Type traveled in each study as well as the predominant trucking operations in the SB and L/SH studies. However, it could be argued that the HVs in the 100-Car Study represent a more diverse population of HVs since they were not limited to L/SH and SB trucks. In fact, 25 different HV types were identified as being involved in LV-HV interactions in the 100-Car Study. Thus, it is likely the results for at-fault HV drivers in the 100-Car Study might be more representative of HV drivers in general, while the results for $\mathrm{HV}$ drivers in the $\mathrm{SB}$ and $\mathrm{L} / \mathrm{SH}$ studies may provide greater insight for these specific operations.

Recall that the Contributing Factors category describes why the incident occurred. The most frequent Contributing Factor across the three studies was Driving Techniques. A breakdown of incidents as a function of the at-fault driver showed that Driving Techniques were primarily attributed to HV drivers. Thus, when the Contributing Factor was known, Driving Techniques was the most frequent Contributing Factor for HV driver at-fault incidents in each of the studies. Similarly, the most frequent Contributing Factors for LV driver at-fault incidents across the three studies were Driving Techniques and Aggressive Driving. These two Contributing Factors accounted for $69.7 \%$ of the LV driver at-fault incidents across the three studies. However, a large proportion of the LV driver at-fault incidents in the 100-Car Study involved the Distracted 
Contributing Factor. In fact, the only time a LV driver at-fault incident was coded with the Distracted Contributing Factors was in the 100-Car Study. This is due to the methodological approach used in that only the LVs in the 100-Car Study were instrumented (thereby allowing analysis of the LV drivers' behaviors while driving) while the LVs in both the SB and L/SH studies were not instrumented.

\section{CONCLUSIONS}

The analyses that were conducted with the LV-HV interactions captured in the 100-Car Study provide convincing evidence to support the contention that LV-HV interactions are a serious problem. While the 100-Car Study captured 9,125 critical incidents, only 246 LV-HV interactions (2.7\%) were identified. While $2.7 \%$ may appear to represent a small proportion of the overall critical incident picture, it should be noted that LV-HV interactions have the potential to become serious, and even fatal due to the tremendous difference in weight between an $\mathrm{HV}$ and LV.

There are six key findings that stem from the analyses conducted on the interactions between HVs and LVs. First, of the 246 interactions that were analyzed, 138 (56.1\%) were assessed to have been the fault of the LV driver. HV drivers were at-fault in 79 (32.1\%) of the incidents, while in the remaining 29 (11.8\%) incidents, it was unknown if the HV or LV driver was at-fault. Excluding the incidents where it was unknown if the HV or LV driver was at-fault, $63.6 \%$ and $36.4 \%$ of the incidents were the fault of the LV and HV drivers, respectively. Thus, LV drivers were judged to have been responsible for a substantial proportion of the LV-HV interactions. These findings support what the drivers in the Hanowski et al. (1998) focus groups reported about LVs being their most important safety concern. Further, the results are similar to prior published studies that used a crash database approach to assess LV-HV interactions (cf. Blower, 1998; Stuster, 1999; Wang, Knipling, and Blincoe, 1999). Based on these findings, it is suggested that focusing on the LV driver, and their errors, may provide the largest area of opportunity for reducing such events.

The second important finding from these analyses was in regard to the different Incident Types that were frequent among HV and LV drivers. For LV driver at-fault incidents, the most frequent Incident Types were: Late Braking for Stopped/Stopping Traffic (41.3\%), Lane Change Without Sufficient Gap (21.7\%), and Aborted Lane Change (8\%). These particular Incident Types are indicative of at-risk driving behaviors. Once again, the objective data support the sentiment of the L/SH drivers in the Hanowski et al. (1998) focus group who indicated that during their daily travel, they were often "cut-off” by LV drivers. In addition, the data supports the results from the L/SH on-road study (Hanowski, Keisler, and Wierwille, 2004) where the most prevalent Incident Type for LV driver at-fault incident was Lane Change Without Sufficient Gap (accounting for $24.8 \%$ of the LV driver at-fault incidents in the L/SH study). In contrast, the most frequent Incident Types for $\mathrm{HV}$ at-fault drivers were: Lane Change Without Sufficient Gap (26.6\%), Lateral Deviation of Through Vehicle (21.5\%), and Left Turn Without Clearance (13.9\%).

The third finding is the difference in the Primary Maneuvers for HV and LV drivers. The most frequent Primary Maneuvers for LV driver at-fault incidents were: Braking (32.6\%), Stopped 
(21.7\%), and Changing Lanes (16.7\%). The two most frequent Primary Maneuvers for LV driver at-fault incidents involved assumed difficulties on the part of the LV driver decelerating or stopping. In contrast, the most frequent Primary Maneuvers for $\mathrm{HV}$ driver at-fault incidents were: Changing Lanes (32.9\%), Crosses Over Lane Line (20.3\%), and Left Turn (15.2\%). The two most frequent Primary Maneuvers for HV driver at-fault incidents involved difficulties changing or crossing over the lane line while the vehicle was in motion. These results make intuitive sense because HV drivers have limited visibility and deal with blind spots thereby making lane changes difficult in traffic.

The fourth important finding is related to the Contributing Factors that were most frequent with LV and HV drivers. For LV drivers, the most frequent Contributing Factors for at-fault incidents were: Driving Techniques (70.3\%), Distracted (22.5\%), and Aggressive Driving (22.5\%). The most frequent Contributing Factors for HV driver at-fault incidents were: Unknown (68.4\%), Driving Techniques (15.2\%), and Distracted (11.4\%). The large number of Unknown Contributing Factors for HV driver at-fault incidents is indicative of the methodology used to code these events. Because the HV did not have any video cameras, the Contributing Factor was coded with respect to the behaviors of the LV driver. As the LV driver was not responsible for the incident, it was unlikely they would be coded with a Contributing Factor, thus the high frequency of Unknown Contributing Factors. Further, the methodology used to code the Contributing Factors also explains the similarities between LVs and HVs (i.e., they were all coded with respect to the LV driver, and therefore, they should be similar).

The fifth noteworthy finding from the current research involves the Accident Types (using the LTCCS approach and terminology) that were most prevalent for LV and HV drivers. The most prevalent Accident Types for LV driver at-fault incidents were: Scenarios 20/21: Same Trafficway/Same Direction: Rear End: Approaches Stopped Vehicle (26.8\%); 38/39: Same Trafficway/Same Direction: Forward Impact: Avoid Collision with Vehicle (22.5\%); and 28/29: Same Trafficway/Same Direction: Rear End: Approaches Decelerating Vehicle (17.4\%). Approximately 55\% of the LV driver at-fault incidents involved a Rear End approach. These Accident Types also support the findings from the analysis of the most prevalent Primary Maneuvers for LV driver at-fault incidents: decelerating or stopped. Conversely, the most prevalent Accident Types for HV driver at-fault incidents were: Scenarios 44/45: Same Trafficway/Same Direction: Forward Impact: Sideswipe Angle: In Blind Spot (27.7\%); 38/39: Same Trafficway/Same Direction: Forward Impact: Avoid Collision with Vehicle (15.2\%); and 25/25: Same Trafficway/Same Direction: Rear End: Approaches Constant Speed Vehicle (8.9\%). Approximately $42 \%$ of the HV driver at-fault incidents involved a Sideswipe Angle. These Accident Types also support the findings from the most prevalent Primary Maneuvers for HV driver at-fault incidents: changing lanes and crossing the lane line.

The sixth noteworthy finding from the current research reflects some of the similarities and differences found between the current study and prior studies using a crash database approach in analyzing $\mathrm{LV}-\mathrm{HV}$ interactions. While both approaches found that $\mathrm{LV}$ drivers were responsible for the majority of LV-HV interactions, the reasons why these interactions occurred differed with respect to the methodologies used to assess these interactions. For example, the current research found that $22.5 \%$ of the LV driver at-fault incidents were cited with the Contributing Factors of Aggressive Driving. In Stuster's (1999) analysis, only 4.3\% of the LVs were cited with the 
driver-related factor "Erratic/Reckless Driving" (it should be noted that Stuster assessed only fatal crashes). Moreover, Hankey et al. (1999) found that $31.1 \%$ of the fatal crashes in the FARS database were cited with Aggressive Driving. The results from the current study (22.5\%) are within the range reported by Stuster (4.3\%) and Hankey (31.1\%).

The current research also found that $41.8 \%$ of the $\mathrm{HV}$ driver at-fault incidents involved a Sideswipe Angle, while $55.1 \%$ of the LV driver at-fault incidents involved a Rear End approach. These results differed from Blower's (1998) review of fatal LV-HV crashes. He found that 9.4\% of fatal LV-HV interactions, where only the HV driver was cited with a driver related factor, involved a sideswipe angle. Further, Blower's (1998) analysis found that $13.9 \%$ of the fatal LV$\mathrm{HV}$ interactions, where only the $\mathrm{LV}$ driver was cited with a driver-related factor, involved a rearend strike. When Council et al. (2003) reviewed all types of LV-HV crashes in North Carolina, they found that $23.2 \%$ of the HV driver at-fault crashes involved a sideswipe and $28.5 \%$ of the LV driver at-fault crashes involved a rear-end approach. These discrepancies might highlight the differences between analyzing crashes and near crashes and/or the methodologies used analyze the data (i.e., a crash database approach versus a naturalistic or in situ data collection approach).

The results of the current study in conjunction with Hanowski, Keisler, and Wierwille (2004) indicated that LV-HV interactions represent a serious problem. While there were several differences across the three studies, the results consistently showed that LV drivers are more likely to be responsible for the LV-HV interaction than $\mathrm{HV}$ drivers. It is believed that the results from the 100-Car, SB, and L/SH studies provide a more complete description of the LV-HV interaction picture. Furthermore, the comparisons among these three studies address the limitations of not having both an instrumented LV and HV. 


\section{CHAPTER 1: LIGHT VEHICLE - HEAVY VEHICLE INTERACTIONS COLLECTED IN THE 100-CAR STUDY}

\section{INTRODUCTION}

\section{Overview of the Light Vehicle-Heavy Vehicle Problem}

Truck crashes represent a significant problem on our highways. In 2002, 434,000 large trucks (gross weight $\geq 10,000 \mathrm{lbs}$ ) were involved in vehicle crashes; 4,542 of these crashes resulted in a fatality. A total of 4,897 people died and an additional 210,000 were injured. Large trucks accounted for $4 \%$ of all registered vehicles in 2002, yet represented $8 \%$ of all vehicles involved in fatal crashes (National Highway Traffic Safety Administration, NHTSA, 2003). Truck crashes and their associated injuries and fatalities cost an estimated 24.4 billion in direct and indirect costs in 2002 (FMCSA, 2002).

The disproportionate number of vehicles to fatalities among large trucks is likely to contribute to the perception that truck drivers are irresponsible. However, these data do not signify that truck drivers are necessarily the problem. In fact, truck drivers have lower non-fatal crash rates per million vehicle miles traveled than light vehicles (NHTSA, 2003). However, light vehicles are extremely vulnerable when they interact with trucks because trucks often weigh 20-30 times as much as light vehicles (Insurance Institute for Highway Safety, 2002), and trucks take 20-40\% farther to stop than light vehicles (NHTSA, 1987). This is best illustrated by the fact that over three-fourths of multiple vehicle fatal truck crashes resulted in the occupant(s) of the other vehicle being killed (NHTSA, 2004).

To combat this problem, proposals have been made to separate light and heavy vehicles on highvolume roads. For example, STAR Solutions has proposed to separate heavy trucks from passenger traffic on Interstate 81 (http://www.virginiadot.org/infoservice/resources/is-I-81-Starexec.pdf), thereby reducing the likelihood of light vehicle-heavy vehicle (LV-HV) interactions. However, the enormous cost and logistical difficulties associated with new and modified road construction suggest that, in most cases, these vehicles will have to share the road for the foreseeable future. Thus, a better understanding of LV-HV interactions is needed to develop alternative interventions and countermeasures directed at reducing and/or eliminating the problem.

Prior Research on Light Vehicle-Heavy Vehicle Interactions

When Hanowski, Wiewille, Gellatly, Early, and Dingus (1998) conducted focus groups with local/short-haul truck drivers, they found that participants ranked "problems with light vehicles" as the most important safety issue. In fact, this was the only safety issue that was consistently cited in all 11 focus groups that were conducted. Similarly, Neale et al. (1998) found that LV$\mathrm{HV}$ interactions were a significant safety concern among a sample of long-haul truck drivers. Only recently has empirical evidence supported truck drivers' claims.

Blower (1998) analyzed the University of Michigan Transportation Research Institute's “Trucks Involved in Fatal Accidents" database for all two-vehicle, truck-passenger vehicle fatal crashes in 1994 and $1995(n=5,453)$. He found that truck drivers were cited with a driver-related factor 
in $26.5 \%$ of the fatal crashes, while passenger vehicle drivers were cited in over $80 \%$ of the fatal crashes. The passenger vehicle driver was the only driver cited in $70.3 \%$ of the fatal crashes, while truck drivers were the only driver cited in $16.2 \%$ of the fatal crashes.

Stuster (1999) found similar results when he reviewed the U.S. Department of Transportation's Fatality Analysis Reporting System. He found that truck driver-related factors were cited in 29\% of fatal truck crashes involving a passenger vehicle, while $67 \%$ of these same interactions were cited as passenger vehicle-related. Moreover, Wang, Knipling, and Blincoe (1999) found that LVs were the initiators in LV-HV fatal crashes by a ratio of approximately 3:1. Thus, it appears the actions of LVs are responsible for a substantial amount of the fatal LV-HV interactions.

Council, Harkey, Nabors, Khattack, and Mohamedshah (2003) took a different approach when they analyzed 16,264 LV-HV interactions from the North Carolina Highway Safety Information System. Rather than examining the police reports from fatal LV-HV interactions, they examined the police reports of both crashes and fatal crashes. While the prior studies assessed the most severe crashes, the Council et al. (2003) study assessed the overall LV-HV crash picture. Contrary to the other studies, however, Council et al. (2003) found that the truck driver was assigned fault in $48 \%$ of the crashes, while the passenger vehicle driver was assigned fault in $40.2 \%$ of the crashes (8.9\% of the crashes were assigned fault to both drivers, while $2.9 \%$ were assigned fault to neither driver). The Council et al. (2003) data suggests that HV drivers were responsible for the majority of the LV-HV interactions (for all types of crashes). Thus, there appears to be some inconsistencies in the literature regarding assigned culpability in LV-HV interactions.

These prior studies assessed LV-HV interactions by examining vehicle crash databases that rely on police reports and crash reconstruction. These approaches are generally reliable, but they do have limitations, including witnesses and crash participants can be biased and report conflicting stories; police officers, while often experienced, generally do not receive extensive training in crash reconstruction; and witnesses or crash participants that were severely injured or killed in the crash are unlikely or are unable to effectively persuade the police officer about their side of the crash (also referred to as the "surviving driver" hypothesis).

Blower (1998) acknowledged the surviving driver limitation in his discussion and compared fatal LV-HV interactions with respect to driver survivability. When only the truck driver survived the fatal LV-HV interaction, the LV driver was cited with at least one driver-related factor in $81.9 \%$ of the fatal crashes, while the HV driver was cited in only $24.1 \%$ of the fatal LV-HV crashes. Conversely, when only the LV driver survived the fatal LV-HV interactions, the LV drivers were cited with at least one driver-related factor in $46.7 \%$ of the crashes, while HV drivers were cited in $57.7 \%$ of the crashes.

It would appear that driver survivability does affect which driver is cited in fatal LV-HV crashes. This makes intuitive sense because the surviving driver is able to report their biased account of the event. However, when both LV and HV drivers survive the crash, the LV driver was cited in $74.1 \%$ of the crashes while the HV driver was cited in only 35.5\% of the crashes (Blower, 1998). 
The crash database approach does not necessarily shed light on the full variety of LV-HV interactions because they rely solely on crashes and fatal crashes. While LV drivers have been shown to be culpable in a significant proportion of LV-HV interactions (Wang, Knipling, and Blincoe, 1999), we do not know why. An alternative approach, and the method used in the current study, is to study the pre-event behaviors of all LV-HV interaction critical incidents, including crashes, near-crashes, and crash-relevant conflicts.

Because the focus of this research is on analyzing critical incidents, it is important to define the three categories that are of most interest: crashes, near-crashes, and crash-relevant conflicts. In the 100-Car Study, Dingus et al. (2004) defined crashes, near-crashes, and crash relevant conflicts as follows:

Crash: Any contact with an object, either moving or fixed, at any speed in which kinetic energy is measurably transferred or dissipated, and includes other vehicles, roadside barriers, objects on or off of the roadway, pedestrians, cyclists, or animals.

Near-Crash: Any circumstance that requires a rapid, evasive maneuver by the subject vehicle, or any other vehicle, pedestrian, cyclist, or animal to avoid a crash. A rapid, evasive maneuver is defined as a steering, braking, accelerating, or any combination of control inputs that approaches the limits of the vehicle capabilities. As a guide: Subject vehicle braking $>0.5 \mathrm{~g}$ or steering input that results in a lateral acceleration $>0.4 \mathrm{~g}$ to avoid a crash constitutes a rapid maneuver.

Crash-Relevant Conflict (Incident): Any circumstance that requires a crash avoidance response on the part of the subject vehicle, any other vehicle, pedestrian, cyclist, or animal that is less severe than a rapid evasive maneuver (as defined above), but greater in severity than a "normal maneuver" to avoid a crash. A crash avoidance response can include braking, steering, accelerating, or any combination of control inputs. A "crash avoidance response" for the subject vehicle is defined as a control input that falls outside of the $99 \%$ confidence limit for control input as measured for the same subject.

Hanowski, Keisler, and Wierwille (2004) assessed two on-road in situ data collection efforts, one involving local/short-haul (L/SH) trucking and the other long-haul trucking with drivers who used sleeper berths, to examine critical incidents that occurred between LVs and HVs. In this study, critical incidents were defined as crashes and near-crashes. Near-crashes were events resulting in a close call or requiring rapid action by a driver to avoid a crash. Video and nonvideo data collected during the two studies were used to characterize 210 critical incidents involving LV-HV interactions. Of the 210 critical incidents analyzed in the Hanowski, Keisler, and Wierwille (2004) study, 78\% were assessed to have been initiated by the LV driver, while the remaining 22\% were initiated by the HV driver. It should be noted that in Hanowski, Keisler, and Wierwille (2004), "initiate" is synonymous with "at-fault." Thus, a vehicle that initiated an incident is meant to reflect the vehicle that was at-fault or responsible for the incident.

The benefits of the naturalistic data collection approach used in Hanowski, Keisler, and Wierwille (2004) are three-fold: (1) video and other supporting data are collected before, during, 
and after the event occurs, thereby providing a complete picture of the incident as it unfolds; (2) various types of non-crash LV-HV interactions can be analyzed; and (3) the use of video and non-video data allowed one to make objective assessments on the critical reason(s) for the incident (rather than incomplete, subjective police reports).

However, one limitation of this approach was that the video cameras were only installed in the HVs and not the LVs. Therefore, Hanowski, Keisler, and Wierwille (2004) were only able to assess LV-HV interactions from the HV driver's perspective. Thus, it is possible they missed critical incidents that were only apparent from the LV driver's perspective. Furthermore, the lack of instrumentation in LVs limits the understanding of the LV driver's behavior during the incident.

These limitations were addressed in the 100-Car Study by installing video cameras on LVs (Dingus et al., 2004). All identified LV-HV interactions from the 100-Car data set were included in the current analyses. Together, results from the current study and the Hanowski, Keisler, and Wierwille (2004) study may provide a more complete picture of the LV-HV interaction problem.

The current study used two classification methodologies to assess all LV-HV interactions: the classification methodology used in Hanowski, Keisler, and Wierwille (2004) (originally developed by Wierwille et al., 2001), and the methodology and terminology from the Large Truck Crash Causation Study (LTCCS) (Thieriez, Radja, and Toth, 2002). Thus, all LV-HV interactions in the current data set were coded with two similar, yet distinct, classification approaches. The primary advantage of this method is that reliable and valid comparisons can be made with both prior and future research studies using either approach.

\section{Research Goals}

The data from the 100-Car Study (Dingus et al., 2004) were used in the current project to assess the LV-HV interaction problem from the LV drivers' perspective. There were four primary aims in the current effort:

- Gain a better understanding of LV-HV interactions on our nation's roadways.

- Continue to develop the classification scheme and corresponding Contributing Factors list for LV-HV interactions used in Hanowski, Keisler, and Wierwille (2004) and use the terminology and methodology described in the LTCCS (Thieriez, Radja, and Toth, 2002).

- Compare the current data to the data obtained in the Hanowski, Keisler, and Wierwille (2004) study for a more complete picture of the LV-HV interaction problem.

- Provide background information that would serve as a necessary prerequisite to the development of countermeasures for LV-HV interactions.

\section{METHOD}

\section{Participants and Setting}

One hundred participants who commute to and from the Washington, DC metro area were initially recruited as drivers in the 100-Car Study (Dingus et al., 2004). As some participants had to be replaced for various reasons (e.g., dropped out of the study because they moved from the 
area), the final number of participants was 109. Age and number of miles driven annually were used to select a subject population that would increase the probability that rear-end collisions would occur in 13 months of data collection. High mileage drivers were selected to increase the number of vehicle miles traveled per year (increase exposure). Greater number of younger drivers (ages 18 through 25) were recruited as they are overly represented in rear-end collisions as compared to other age groups. Also, more males than females were recruited since males are overly represented in rear-end crashes (Knipling, Wang, and Yin, 1993). It should be noted that participants were recruited from all age groups and that the target average annual mileage per year was approximately 27,000 miles/year. However, the actual mileage driven by participants in the 100-Car Study did not match their self-reported annual mileage prior to the study. The actual mileage of participants in the 100-Car Study can be found in Dingus et al. (2004). Table 1 , shown below, displays the age and gender distribution of participants.

Table 1. Participant Age and Gender Distributions.

\begin{tabular}{|l|c|c|c|}
\hline \multirow{2}{*}{ Age } & \multicolumn{2}{|c|}{ Gender } & \multirow{2}{*}{ (N and \% of Total) } \\
\cline { 2 - 3 } & Female & Male & \\
\hline $18-20$ & 9 & 7 & 16 \\
& $8.3 \%$ & $6.4 \%$ & $14.7 \%$ \\
\hline $21-24$ & 11 & 10 & 21 \\
& $10.1 \%$ & $9.2 \%$ & $19.3 \%$ \\
\hline $25-34$ & 7 & 12 & 19 \\
& $6.4 \%$ & $11.0 \%$ & $17.4 \%$ \\
\hline $35-44$ & 4 & 16 & 20 \\
& $3.7 \%$ & $14.7 \%$ & $18.4 \%$ \\
\hline $45-54$ & 7 & 13 & 20 \\
& $6.4 \%$ & $11.9 \%$ & $18.3 \%$ \\
\hline $55+$ & 5 & 8 & 13 \\
& $4.6 \%$ & $7.3 \%$ & $11.9 \%$ \\
\hline \multirow{2}{*}{ Total Percentage } & $39.4 \%$ & $60.6 \%$ & $100.0 \%$ \\
\hline
\end{tabular}

\section{Light Vehicle Types}

The data that were collected in the 100-Car Study came from six makes/models/years of LVs, including Toyota Camry (1997-2001), Toyota Corolla (1993-2002), Ford Explorer (1995-2000), Ford Taurus (2000-2002), Chevrolet Malibu (2002), and Chevrolet Cavalier (2002). The Toyota and Ford models were chosen based on recent sales figures and on the number of vehicles available in the Washington, DC area. Figures 1 and 2 show examples of the LVs used in the 100-Car Study. 


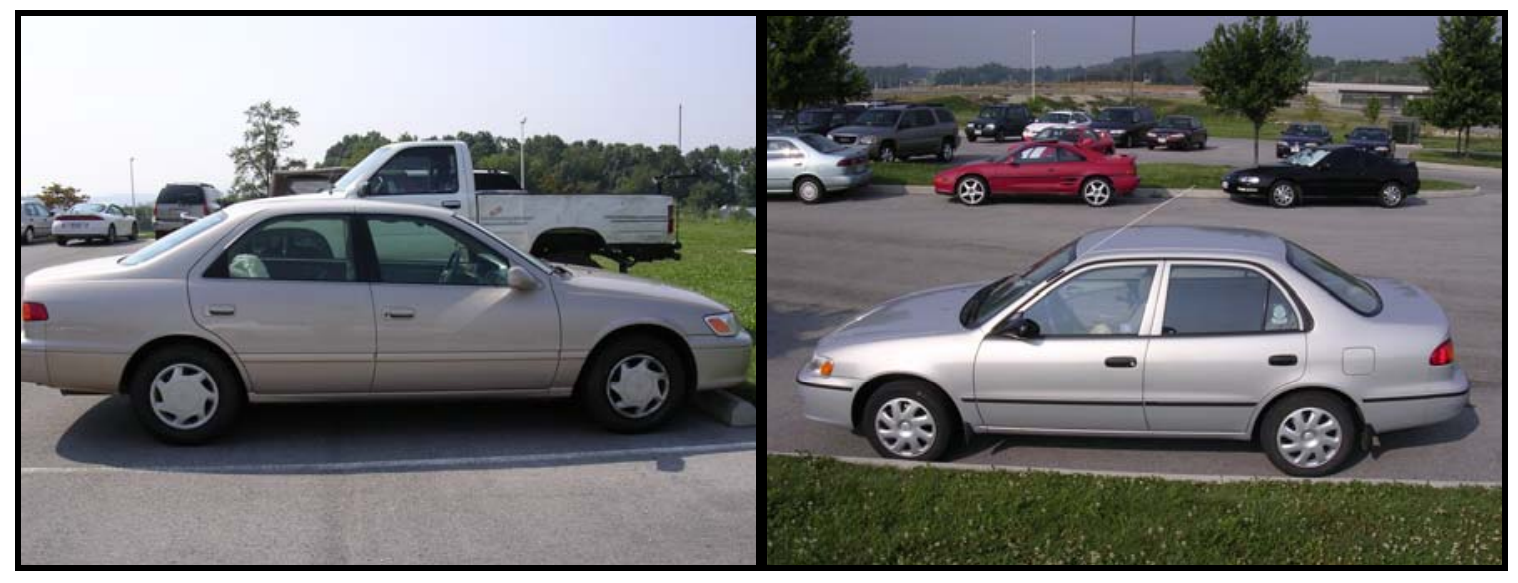

Figure 1. Toyota Camry and Toyota Corolla Used in 100-Car Study.

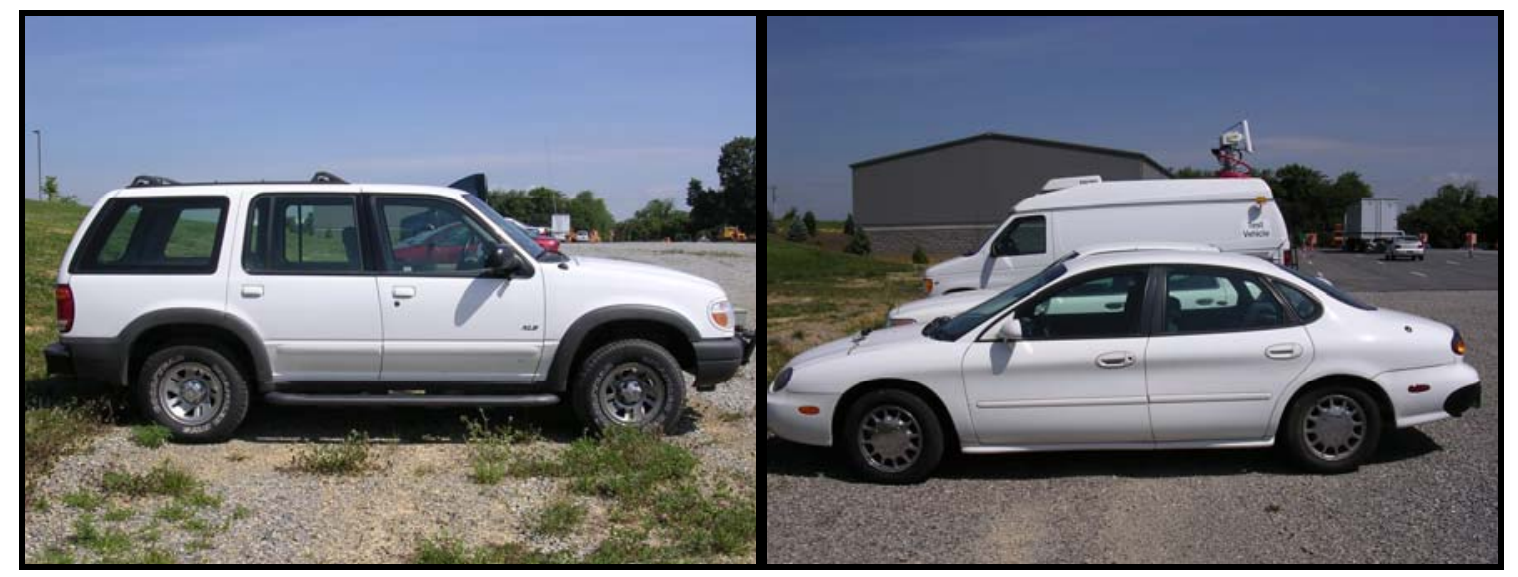

Figure 2. Ford Explorer and Ford Taurus Used in 100-Car Study.

A total of 20 Chevrolet vehicles (10 Malibus and 10 Cavaliers) were leased from the Virginia Tech Motor Pool and were instrumented with data collection equipment. Twenty participants were given leased vehicles to drive for one year. The additional 80 vehicles (comprised of the aforementioned Toyota and Ford models) were the participants' personal vehicles. These vehicles were instrumented with the identical data collection systems as the leased vehicles.

\section{Data Collection Methodology for the 100-Car Study}

A full description of the research methodology used in the on-road portion of the 100-Car Study can be found in Dingus et al. (2004). Because the data used in the current effort consisted of the video recordings of critical incidents, the primary methodological considerations to be described in this report are those related to the video systems.

\section{Video Camera Systems}

As shown in Figure 3, five video cameras were used in the video recording system: (1) a forward-looking camera that captured the forward roadway scene, traffic situation, and possible incidents; (2) a driver's face camera that was used to record facial expressions, eyelid closure, 
glance position, and head turns; (3) a right-side camera was mounted on the A-pillar of the passenger side and faced outward; (4) a dome camera was mounted from inside the vehicle and faced over the driver's shoulder towards the steering wheel, hands, and feet; and (5) a rear camera that was intended to capture the situation behind the vehicle. Infrared lighting was used to illuminate the vehicle cab so that the driver's face as well as their hands could be viewed by the camera during nighttime driving.

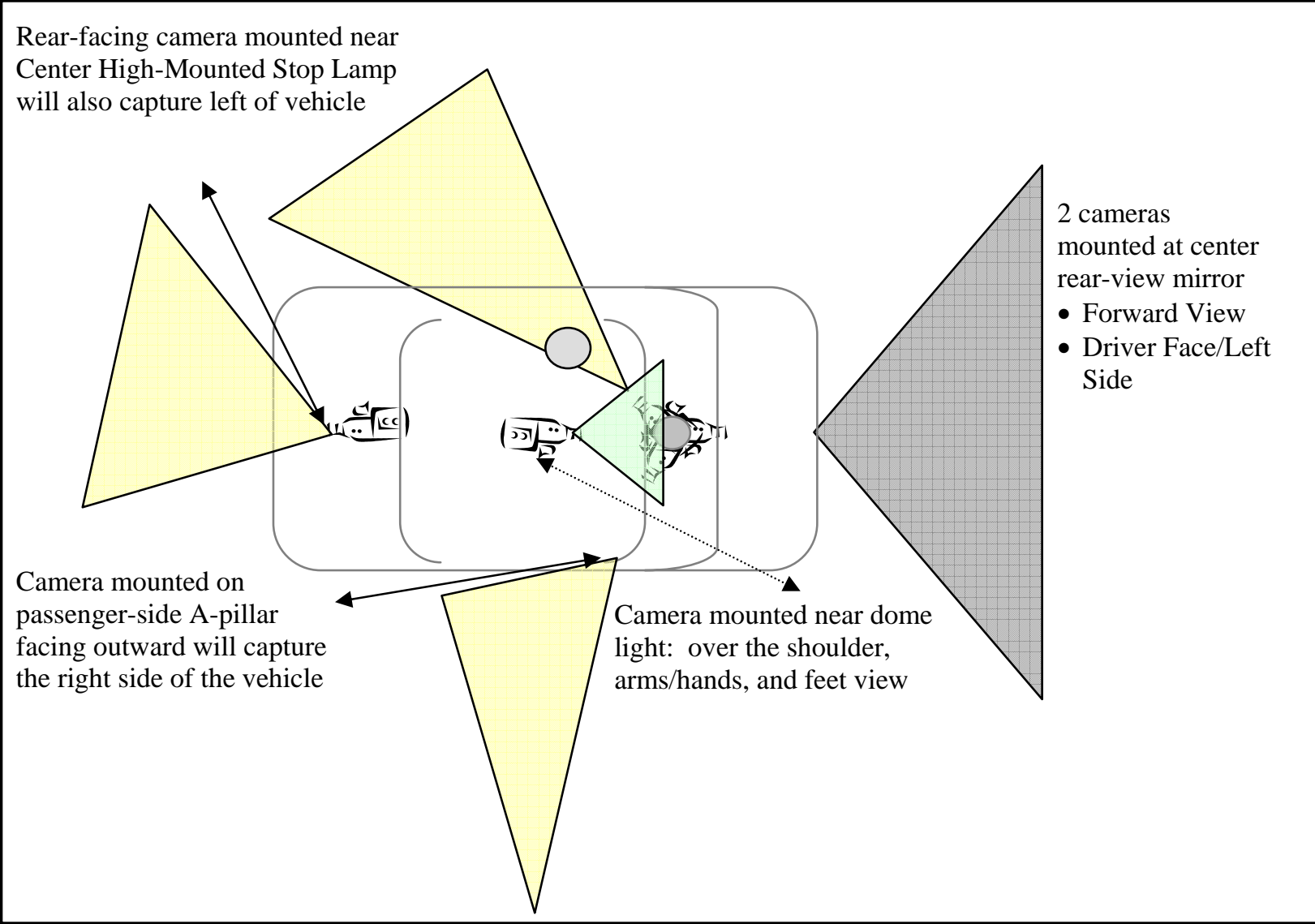

\section{Figure 3. The Five Camera Views Recorded in the Instrumented Vehicle.}

The video camera arrangement shown in Figure 3 had several advantages. First, it provided good coverage around the vehicle so that incidents could be captured as they developed. Second, the driver's facial expression, approximate glance direction, and approximate level of eye closure were also captured. Third, the arrangement provided appropriate views, whether moving forward or backward.

The five camera images were multiplexed into a single image as shown in Figures 4 and 5. Note that the right side camera and the rear camera were presented in the lower left quadrant in a split arrangement. 


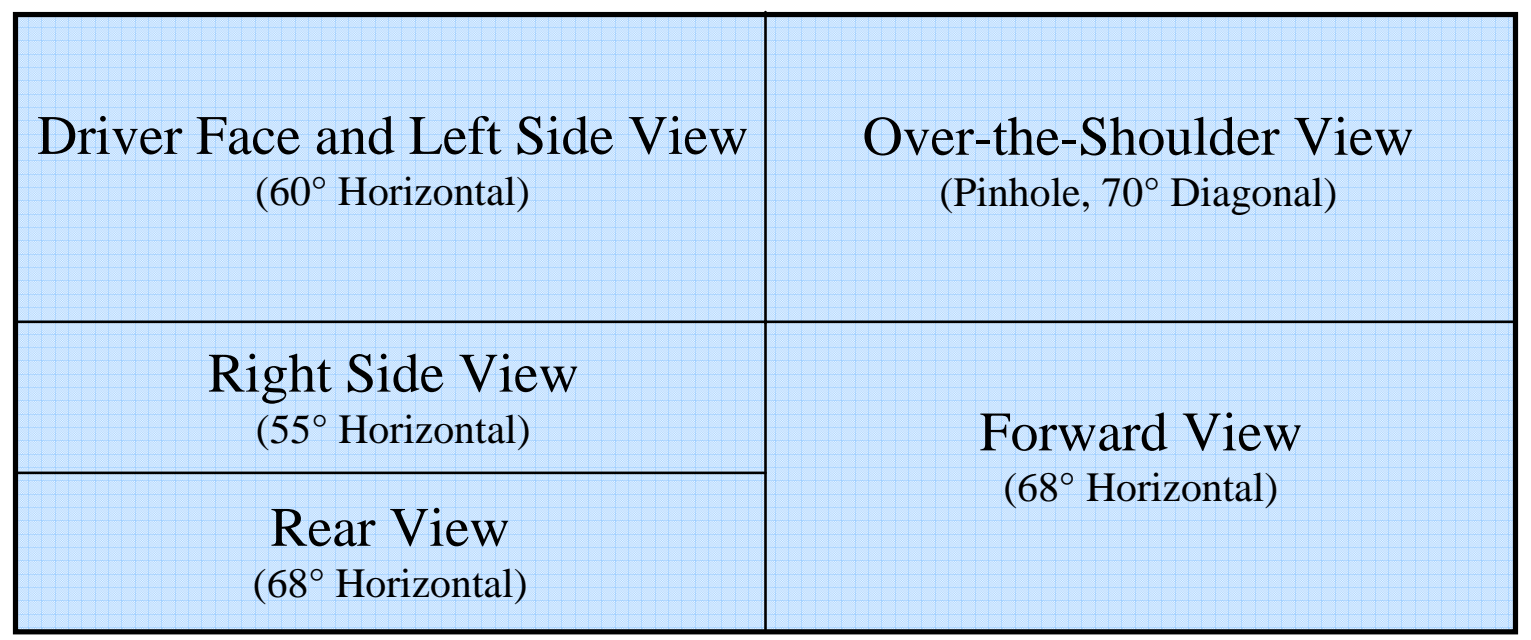

Figure 4. Diagram of the Multiplexed Camera Views.

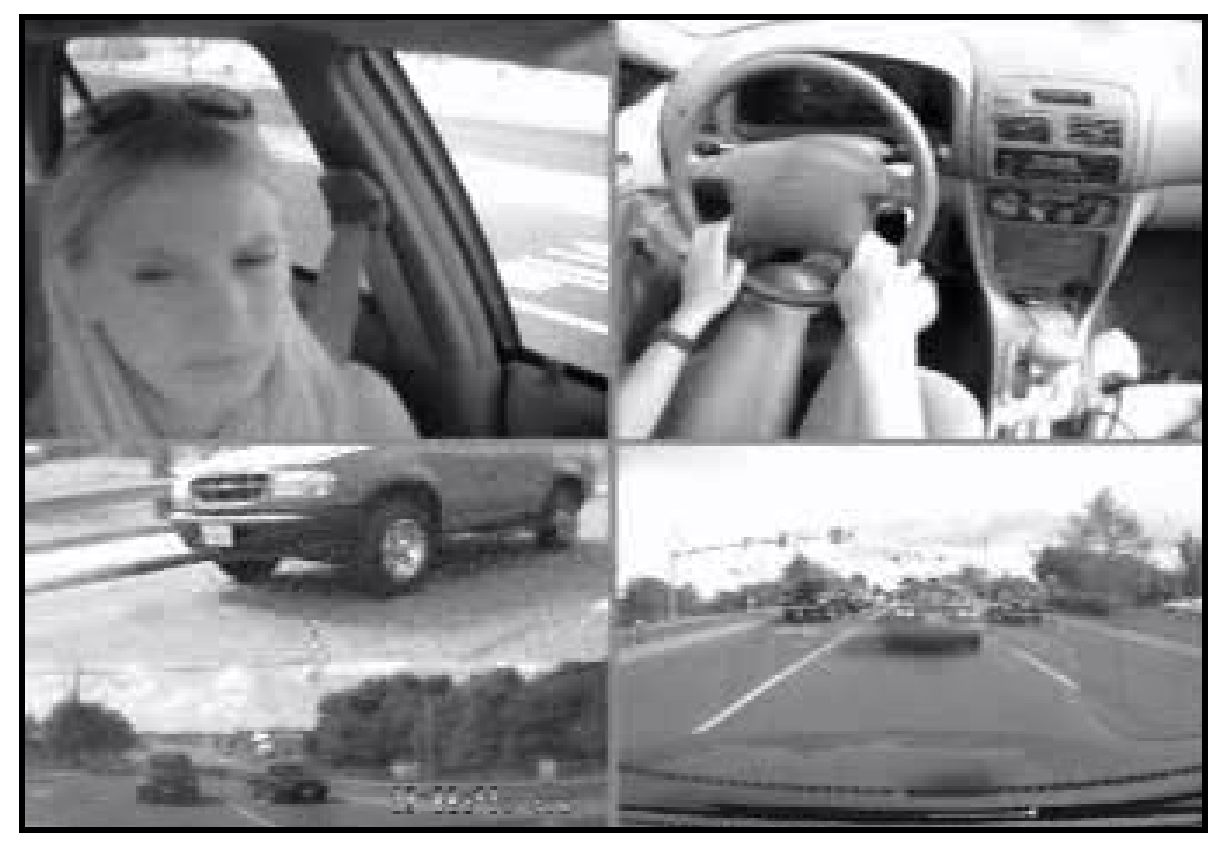

Figure 5. Split-Screen Presentation of the Five Camera Views.

\section{Video Recording Operation}

Video recording was tied to the booting/powering system and it began to operate 2 minutes after the ignition was on. It also shut down in an orderly manner when the ignition was turned off. It was desired for the recording system to record for as long as possible without requiring technician/researcher attention. Therefore, multiple recorders designed to operate in sequence were used. The video continuously recorded while the ignition was on, thereby allowing laboratory review and selection of the video without losses of any kind. 
The videotaped episodes/incidents were selected and keyed to digitally recorded data. In some cases, the videotape timestamp was used to access the corresponding digital data. In other cases, the incident flags (described later) in the digital data were used to access the corresponding video. Therefore, there was a straightforward keying procedure that allowed both kinds of access to take place efficiently.

\section{Data Collection and Storage}

"Chase" vehicles drove to pre-determined locations (e.g., parking lots) and downloaded the data from the experimental vehicles via a data transfer cable that connected to an outlet located near the rear license plate. Each chase vehicle had a laptop computer with a large hard drive to store all vehicle data. After each download from the experimental vehicles, the success of the duplication procedure was verified. Once $2.3 \mathrm{~GB}$ of data were downloaded from experimental vehicles, the data were copied to a DVD and verified. This DVD was duplicated; one copy was sent to VTTI and the other copy was kept in Northern Virginia.

As the data arrived at VTTI, they were downloaded to VTTI's network attached storage (NAS) and saved. Once the data was safely copied to the networked attached storage at VTTI and quality checks were performed, the data were then remotely deleted from the experimental vehicle hard drive.

\section{Incident Flag}

A critical incident involves an unexpected event resulting in a close call or requiring rapid action (evasive maneuver) on the part of a driver to avoid a crash. Critical incidents were detected by one of three methods. The first method involved flagging events where the car sensors exceeded a specified value. An example of this is a braking response of $>0.6 \mathrm{~g}$ would be recorded as a potential incident where the driver may have braked in a panic.

Table 2 lists all of the triggers and levels that were used in this first method. The second incident flagging method occurred when the driver pressed an incident pushbutton located on the dashboard. Drivers were instructed to depress a button on the dashboard (after the event, not during the event) if they witnessed an incident or were involved in an incident. The third method of detecting incidents was through analysts' judgments when reviewing the video. Note that the video systems were operational as long as the ignition was turned on. In identifying incidents, analysts looked through epochs flagged from either of the first two methods and could flag additional events within the epoch (termed "user triggered") if an incident was detected visually. Only those events that involved a LV-HV interaction are described in the current analyses. The results of other project analyses can be found in Dingus et al. (2004). 
Table 2. Triggers and Their Levels Used to Identify Critical Incidents in the 100-Car Study Database.

\begin{tabular}{|l|l|}
\hline \multicolumn{1}{|c|}{ Trigger Type } & \multicolumn{1}{c|}{ Description } \\
\hline Lateral Acceleration & Lateral motion equal or greater than $0.7 \mathrm{~g}$. \\
\hline $\begin{array}{l}\text { Longitudinal } \\
\text { Acceleration }\end{array}$ & $\begin{array}{l}\text { Acceleration or deceleration equal or greater than } 0.6 \mathrm{~g} . \\
\text { Acceleration or deceleration equal or greater than } 0.5 \text { coupled with a } \\
\text { forward TTC of } 4 \mathrm{~s} \text { or less. } \\
\text { All longitudinal decelerations between } 0.4 \mathrm{~g} \text { and } 0.5 \mathrm{~g} \text { coupled with a } \\
\text { forward TTC value of } \leq 4 \mathrm{~s} \text { and that the corresponding forward range value } \\
\text { at the minimum TTC is not greater than } 100 \mathrm{ft} .\end{array}$ \\
\hline $\begin{array}{l}\text { Critical Incident } \\
\text { Button }\end{array}$ & $\begin{array}{l}\text { Activated by the driver upon pressing a button located on the dashboard } \\
\text { when an incident occurred that he/she deemed critical. }\end{array}$ \\
\hline collision & $\begin{array}{l}\text { Acceleration or deceleration equal or greater than } 0.5 \text { coupled with a } \\
\text { forward TTC of } 4 \mathrm{~s} \text { or less. } \\
\text { All longitudinal decelerations between } 0.4 \mathrm{~g} \text { and } 0.5 \mathrm{~g} \text { coupled with a } \\
\text { forward TTC value of } \leq 4 \mathrm{~s} \text { and that the corresponding forward range value } \\
\text { at the minimum TTC is not greater than } 100 \mathrm{ft} .\end{array}$ \\
\hline $\begin{array}{l}\text { Rear time-to- } \\
\text { collision }\end{array}$ & $\begin{array}{l}\text { Any rear TTC trigger value of } 2 \mathrm{~s} \text { or less that also has a corresponding } \\
\text { rear range distance of } \leq 50 \mathrm{ft} \text { AND any rear TTC trigger value where the } \\
\text { absolute acceleration of the following vehicle is greater than } 0.3 \mathrm{~g}\end{array}$ \\
\hline Yaw rate & $\begin{array}{l}\text { Any value greater than or equal to a plus AND minus } 4 \text { degree change in } \\
\text { heading (i.e., vehicle must return to the same general direction of travel) } \\
\text { within a } 3 \mathrm{~s} \text { window of time. }\end{array}$ \\
\hline
\end{tabular}

The incident flags (associated with the first and second triggering methods) were computed and detected on-line (as well as stored) with the flag appearing in the video. Since the entire video recording was reviewed, the presence of flags served as an indicator to the analyst of the high likelihood, but not certainty, of an incident occurrence. However, the analyst was also mindful of the possibility of incidents without flags and reviewed the tapes accordingly. The data analysts watched $90 \mathrm{~s}$ epochs (1 min prior and $30 \mathrm{~s}$ post incident) of each driving incident and recorded the information shown in Table 3. 
Table 3. Information Recorded During 90 s Epoch Analysis.

\begin{tabular}{|l|l|}
\hline Event severity & Surface condition \\
\hline Event nature & Traffic flow \\
\hline Event time begin and end & Travel lanes \\
\hline Subject number & Traffic density \\
\hline Pre-incident maneuver & Traffic control \\
\hline Maneuver judgment & Relation to junction \\
\hline Precipitating event & Alignment \\
\hline Driver reaction & Locality \\
\hline Driver behavior & Lighting \\
\hline Driver Impairments & Weather \\
\hline Alcohol use & Wipers \\
\hline Willful behavior & Driver's seat belt \\
\hline Driver proficiency & Surrounding vehicle position \\
\hline Roadway infrastructure & Surrounding vehicle type \\
\hline Driver distraction & Surrounding vehicle maneuver \\
\hline Hands on wheel & Surrounding vehicle reaction \\
\hline Vehicle contributing factors & Fault \\
\hline Visual obstructions & Narrative \\
\hline
\end{tabular}

Data Reduction Reliability

Given that data analysts were asked to perform subjective judgments on the video and driving data, training procedures were implemented to improve both inter- and intra-rater reliability. Reliability testing was then conducted to measure the resultant inter- and intra-rater reliability. First, data analyst managers performed spot checks of the data analysts' work, monitoring both event validity judgments as well as recording all database variables. All data analysts also performed 30 mins of spot-checking of their own or other data analysts' work per week.

To determine how successful these techniques were, an inter- and intra-rater reliability test was conducted during the last three months of data reduction. Three reliability tests were developed (each containing 20 events) for which the data analyst was required to make validity judgments. In each of the three reliability tests, three of the 20 events were also fully reduced by the data analysts. Three of the test events on Test 1 were repeated on Test 2 and three other events were duplicated between Tests 2 and 3, to obtain a measure of intra-rater reliability.

The Kappa statistic was also used to calculate inter-rater reliability (Cicchetti and Feinstein, 1990). The Kappa coefficient $(K=0.65, p<0.0001)$ indicated that the association among raters was significant. The average of the pair-wise correlation coefficients for the inter-rater analysis was 0.86 . The coefficients for the intra-rater analysis were extremely high with nine raters achieving a correlation of 1.0 among the three reliability tests and five raters achieving a correlation of 0.99. Given these three methods of calculating inter-rater reliability, it appears that the data analysts training coupled with spot-checking and weekly meetings proved to be an effective method for achieving high inter-rater and intra-rater reliability. 


\section{Strengths and Limitations of the Methodology Used}

All research approaches have strengths and limitations. Listed below are the strengths and limitations of the approach used in the current study.

$\underline{\text { Strengths }}$

The primary strength of the approach used in this study was that all driver behaviors, visible by way of the video camera, were recorded whenever the vehicle was on and in motion. This information is vital in developing an understanding of the incident, the events leading up to the incident, and the aftermath of the incident from the LV driver's viewpoint. The video camera arrangement described allowed researchers to watch the critical incidents unfold from multiple camera views. The video camera system that was used not only afforded an opportunity to understand what happened, but in many cases why it happened. A second advantage of this approach was that multiple cameras views helped ensure that any critical incidents involving the LV driver were captured and available for analysis.

Possible Limitations There were two possible limitations of the approach used in the current research. First, because the video cameras were installed in the LVs and not the HVs, critical incidents could only be captured from the LV driver's perspective. It was possible that LV-HV interactions, which may have only been apparent from the HV driver's viewpoint, were not recorded. However, because there was fairly complete video recording coverage around the entire LV, it was likely that most $\mathrm{LV}-\mathrm{HV}$ interactions that occurred were recorded.

Second, because there were no cameras mounted in any HV, it is difficult to have a complete understanding of the HV driver's behavior during the incident. The video camera that was directed at the LV driver's face, along with the verbal utterances of the driver, provided the researchers with a fairly complete understanding of the LV driver's behavior before, during, and after each incident. However, this was not the case regarding the behavior of the HV driver. The absence of video footage of the HV driver's face meant that the HV driver's behavior had to be surmised based on the video of the HV collected from the LVs and the comments and facial expressions made by the LV driver.

These limitations were also raised in Hanowski, Keisler, and Wierwille (2004) for the LV-HV analyses that were conducted with both $\mathrm{L} / \mathrm{SH}$ and sleeper berth (SB) trucks. Considering the previous Hanowski, Keisler, and Wierwille (2004) work in conjunction with the current research, a more complete assessment of LV-HV interactions from both the HV and LV driver's perspective was expected. Assumptions regarding driver behavior were required for each of these research efforts, particularly for the driver of the non-instrumented vehicle. 


\section{RESULTS}

\section{Light Vehicle-Heavy Vehicle Interaction Data Set}

The 100-Car Study (Dingus et al., 2004) captured 9,125 incidents. These 9,125 incidents were divided into four categories: LV-LV Interactions, LV-HV Interactions; Single Vehicle Conflicts, and Other. Table 4 provides a description of the different vehicle types in each category.

Table 4. Vehicle Types Captured in the 100-Car Study.

\begin{tabular}{|c|c|c|}
\hline Vehicle Category & \multicolumn{2}{|c|}{ Vehicles Considered in Each Vehicle Category } \\
\hline \multirow{5}{*}{ Light Vehicle } & \multicolumn{2}{|l|}{ Automobile } \\
\hline & \multicolumn{2}{|l|}{ Minivan/Standard Van } \\
\hline & \multicolumn{2}{|l|}{ Motorcycle/Moped } \\
\hline & \multicolumn{2}{|l|}{ Pick-up Truck } \\
\hline & \multicolumn{2}{|l|}{ Sport Utility Vehicle } \\
\hline \multirow{25}{*}{ Heavy Vehicle } & \multirow{4}{*}{ Bus } & Conversion Bus \\
\hline & & Greyhound Bus \\
\hline & & School Bus \\
\hline & & Transit Bus \\
\hline & \multirow{2}{*}{ Emergency Vehicle } & Ambulance \\
\hline & & Fire Truck \\
\hline & \multirow{11}{*}{ Straight Truck } & Straight Truck: Beverage \\
\hline & & Straight Truck: Box \\
\hline & & Straight Truck: Concrete Mixer \\
\hline & & Straight Truck: Dump \\
\hline & & Straight Truck: Flatbed \\
\hline & & Straight Truck: Garbage \\
\hline & & Straight Truck: Multistop/Step Van \\
\hline & & Straight Truck: Other \\
\hline & & Straight Truck: Tow Truck \\
\hline & & Straight Truck: Trailer \\
\hline & & Straight Truck: Unknown \\
\hline & \multirow{7}{*}{ Tractor-Trailer } & Tractor Only \\
\hline & & Tractor-Trailer: Car Carrier \\
\hline & & Tractor-Trailer: Dump Trailer \\
\hline & & Tractor-Trailer: Enclosed Box \\
\hline & & Tractor-Trailer: Flatbed \\
\hline & & Tractor-Trailer: Other \\
\hline & & Tractor-Trailer: Tank \\
\hline & \multicolumn{2}{|c|}{ Construction Equipment } \\
\hline
\end{tabular}


The data set used in the current effort was comprised of a subset of incidents from the 9,125 incidents described above. The 9,125 incidents were reviewed and only those that involved a LV-HV interaction were included in the present analysis.

Figure 6 shows a pie chart of the 9,125 events as a function of the vehicles involved and whether or not the incident was an interaction between vehicles. As can be seen, of the 9,125 events, 246 (2.7\%) involved a LV-HV interaction. In 2003, there were a total of 6,328,000 crashes in the U.S. (NHTSA, 2004). Of these crashes, 313,663 (5\%) were classified as a LV-HV interaction. Thus, the present data set has fewer LV-HV interactions than the national crash statistics.

Of the $246 \mathrm{LV}$-HV recorded incidents, 219 (89\%) were crash-relevant conflicts, 25 (10.2\%) were near crashes, $1(.4 \%)$ was a crash, and $1(.4 \%)$ was undetermined. ${ }^{2}$ For the 79 incidents where the HV driver was judged to have been at-fault, 66 (83.5\%) were crash-relevant conflicts and 13 (16.5\%) were near crashes. For the 138 incidents where the LV driver was judged to have been at-fault, $128(92.8 \%)$ were crash-relevant conflicts, 8 (5.8\%) were near crashes, $1(.7 \%)$ was a crash, and 1 (.7\%) was undetermined. For the 29 incidents where it was unknown if the LV or HV driver was at-fault, 29 (100\%) were crash-relevant conflicts.

\footnotetext{
${ }^{2}$ This was a unique event that was not identified from any of the triggering methods. This event involved the driver reporting that he had received a ticket for illegally passing a stopped school bus. This self-report was then confirmed by reviewing the video.
} 


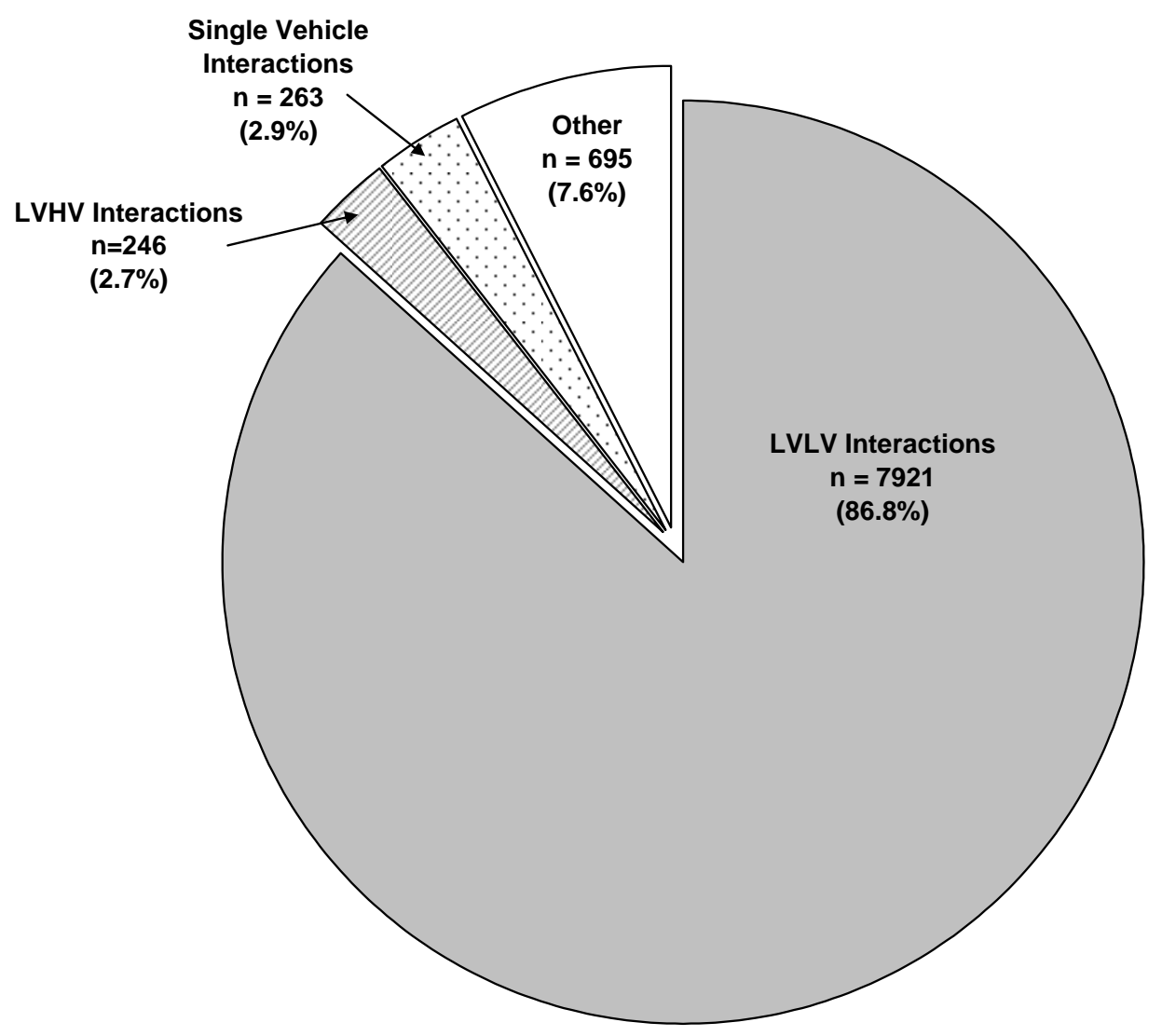

Figure 6. Distribution of the 9,125 Incidents Captured in 100-Car Study.

\section{Incident Types}

Given that the 100-Car data set was comprised of $246 \mathrm{LV}-\mathrm{HV}$ interactions, the next step in the analysis was to determine the vehicles' actions for each incident. To this end, the video and relevant data for each incident were carefully reviewed and then classified as an "Incident Type." Twenty-seven different Incident Types were identified (a detailed description of each is presented in Table 5). It should be noted that the 27 Incident Types listed do not necessarily comprise the entire universe of all types of LV-HV interaction incidents. Rather, the 27 Incident Types listed comprise those that were identified in this data set $(\mathrm{N}=246)$. The Incident Types are written in such a way as to be interchangeable regarding LVs and HVs. Note that this is the same classification strategy outlined in the Hanowski, Keisler, and Wierwille (2004) study. However, in the Hanowski, Keisler, and Wierwille (2004) study, only 20 Incident Types were identified in their data set. 
Table 5. Description of the Incident Types that were Identified in the Current Research.

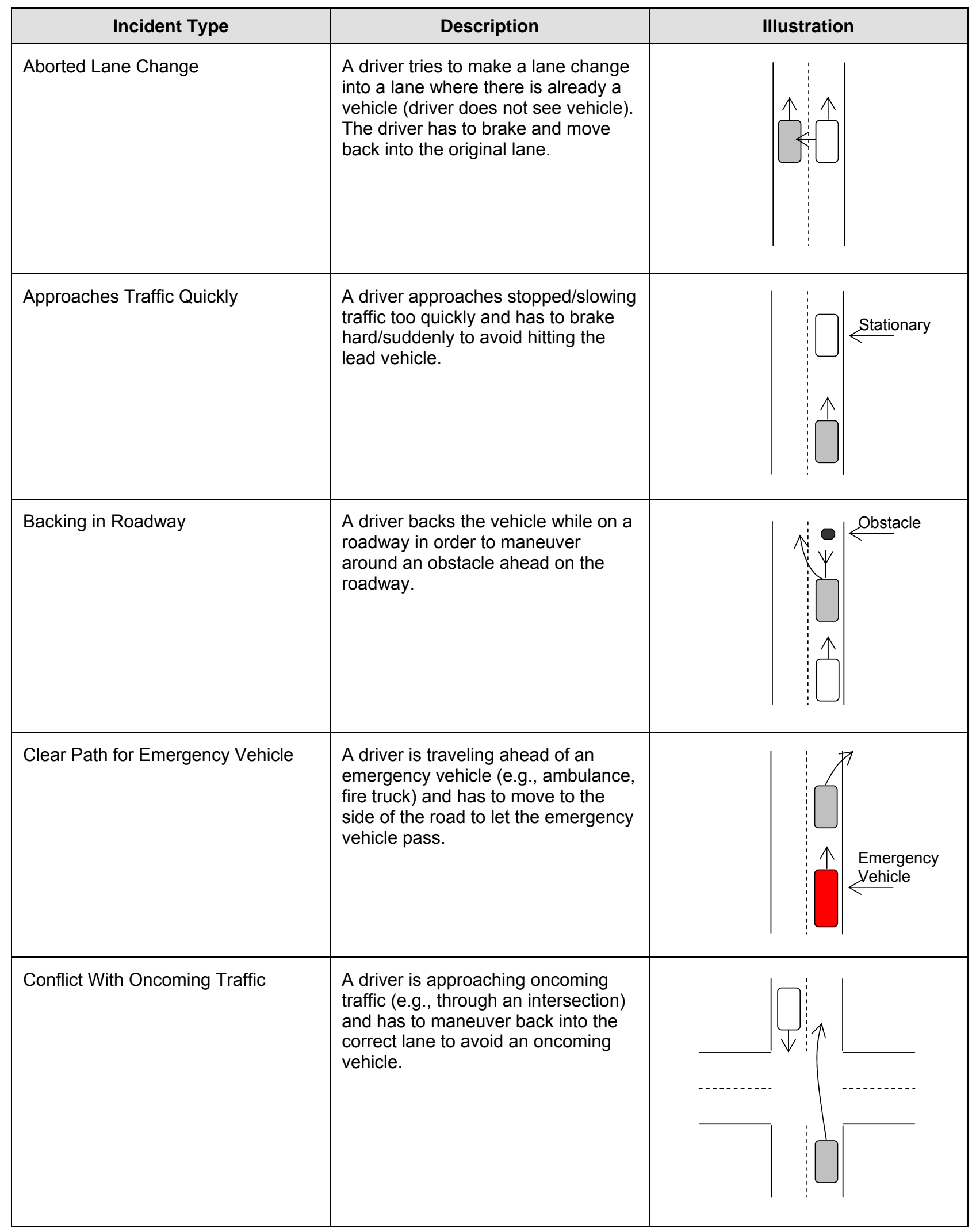




\begin{tabular}{|c|c|c|}
\hline Incident Type & Description & Illustration \\
\hline Following Too Closely & $\begin{array}{l}\text { A driver does not allow adequate } \\
\text { spacing between their vehicle and } \\
\text { the lead vehicle (e.g., tailgating). }\end{array}$ & \\
\hline Improper Lane Change & $\begin{array}{l}\text { A driver makes an improper lane } \\
\text { change with regard to another } \\
\text { vehicle (e.g., does not use signal, } \\
\text { changes lanes behind another } \\
\text { vehicle then does not let vehicle } \\
\text { change lanes, changes lanes across } \\
\text { multiple lanes, etc.) }\end{array}$ & \\
\hline Improper Passing & $\begin{array}{l}\text { A driver passes another vehicle } \\
\text { when it is illegal or unsafe (e.g., } \\
\text { passing across a double yellow line } \\
\text { or without clearance from oncoming } \\
\text { traffic). }\end{array}$ & \\
\hline Improper Stopping at an Intersection & $\begin{array}{l}\text { A driver does not stop appropriately } \\
\text { at the white stop line at an } \\
\text { intersection. }\end{array}$ & \\
\hline Improper U-Turn & $\begin{array}{l}\text { A driver makes a U-turn in the } \\
\text { middle of the road (over the double } \\
\text { yellow line) and blocks traffic in the } \\
\text { opposite direction. }\end{array}$ & \\
\hline
\end{tabular}




\begin{tabular}{|c|c|c|}
\hline Incident Type & Description & Illustration \\
\hline $\begin{array}{l}\text { Improperly Covered Debris From } \\
\text { Lead Vehicle }\end{array}$ & $\begin{array}{l}\text { Debris is blown from the lead vehicle } \\
\text { and obstructs driver's view in the } \\
\text { following vehicle. }\end{array}$ & \\
\hline Lane Change Without Sufficient Gap & $\begin{array}{l}\text { A driver enters an adjacent lane } \\
\text { without allowing adequate space } \\
\text { between the driver's vehicle and the } \\
\text { vehicle ahead/behind it. }\end{array}$ & \\
\hline $\begin{array}{l}\text { Late Braking for Stopped/ Stopping } \\
\text { Traffic }\end{array}$ & $\begin{array}{l}\text { A driver fails to slow in advance for } \\
\text { stopped or stopping traffic and must } \\
\text { brake abruptly. }\end{array}$ & Stationary \\
\hline Lateral Deviation of Through Vehicle & $\begin{array}{l}\text { A driver has substantial lateral } \\
\text { deviation of a through vehicle. } \\
\text { Vehicle may or may not deviate from } \\
\text { the lane. }\end{array}$ & \\
\hline Left Turn Without Clearance & $\begin{array}{l}\text { A driver turns left without adequate } \\
\text { clearance from either oncoming } \\
\text { through traffic or cross traffic from } \\
\text { the left. The driver crosses another } \\
\text { driver's path while entering an } \\
\text { intersecting roadway. }\end{array}$ & \\
\hline
\end{tabular}




\begin{tabular}{|c|c|c|}
\hline Incident Type & Description & Illustration \\
\hline Merge Without Sufficient Gap & $\begin{array}{l}\text { A driver merges into traffic without a } \\
\text { sufficient gap to either the front or } \\
\text { back of one or more vehicles. }\end{array}$ & \\
\hline Obstruction in Roadway & $\begin{array}{l}\text { A stationary object blocks through } \\
\text { traffic, such as traffic that is backed } \\
\text { up or an animal in the roadway. }\end{array}$ & \\
\hline $\begin{array}{l}\text { Roadway Entrance Without } \\
\text { Clearance }\end{array}$ & $\begin{array}{l}\text { A driver turns onto a roadway } \\
\text { without adequate clearance from } \\
\text { through traffic. }\end{array}$ & \\
\hline School Bus Passing Violation & $\begin{array}{l}\text { A driver fails to stop for a stopped } \\
\text { school bus with the stop arm } \\
\text { extended. }\end{array}$ & \\
\hline Slow Speed & $\begin{array}{l}\text { A driver is traveling at a much slower } \\
\text { speed than the rest of the traffic, } \\
\text { causing following traffic to pass the } \\
\text { slow vehicle to avoid a conflict. }\end{array}$ & \\
\hline
\end{tabular}




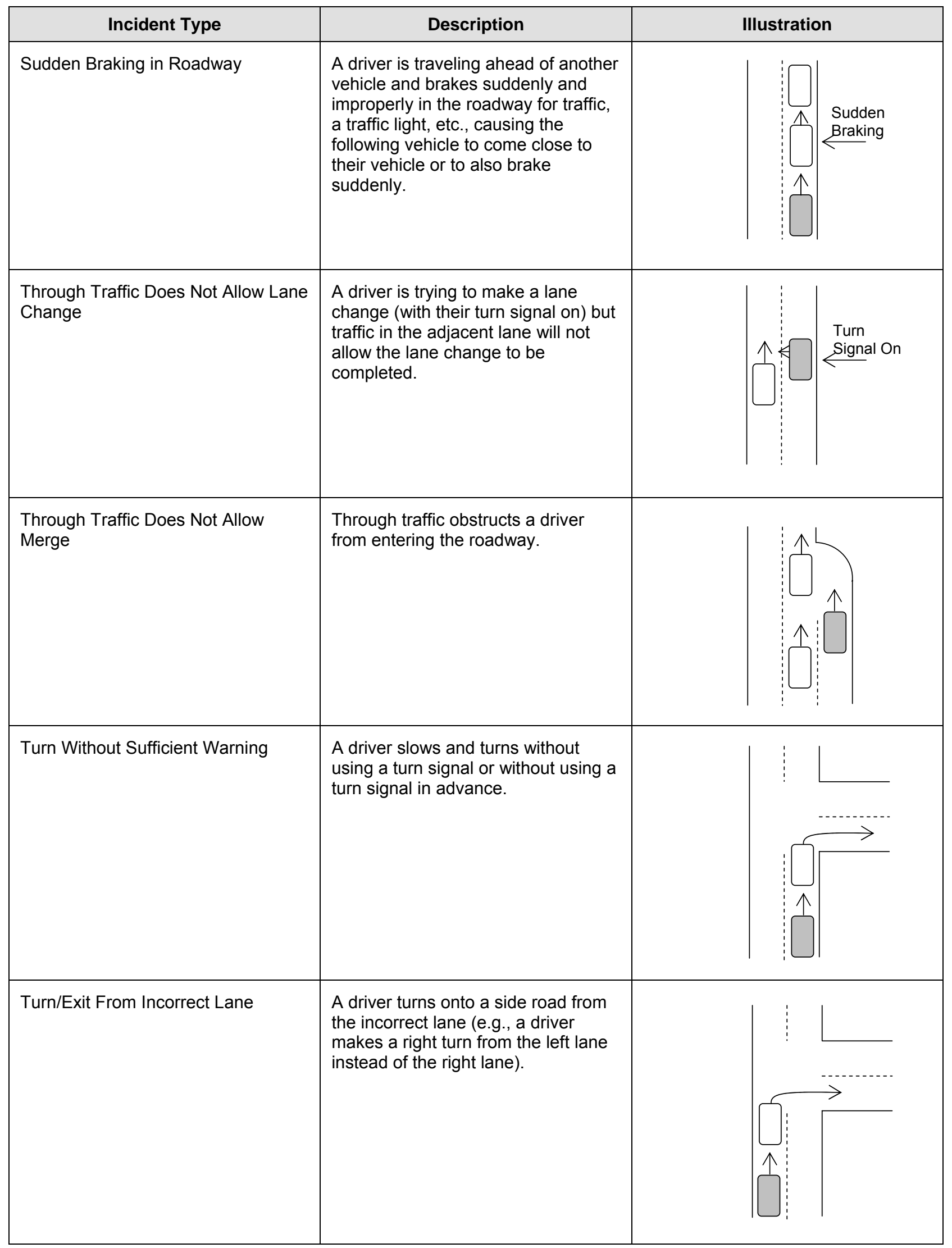




\begin{tabular}{|l|l|}
\hline \multicolumn{1}{|c|}{ Incident Type } & \multicolumn{1}{c|}{ Description } \\
\hline Wide Turn Into Adjacent Lane & $\begin{array}{l}\text { A vehicle partially enters an adjacent } \\
\text { lane when turning. Traffic in the } \\
\text { adjacent lane may be moving in the } \\
\text { same or opposite direction. }\end{array}$ \\
\hline Unable to Determine & $\begin{array}{l}\text { It is not possible to determine which } \\
\text { vehicle is at fault, therefore, it is not } \\
\text { possible to assign an incident type to } \\
\text { the event. }\end{array}$ \\
\hline
\end{tabular}

Table 6 shows the frequency, percentage, and rank ordering for the Incident Types across the entire 100-Car data set. The rank ordering highlights the frequency of Incident Types from most frequently occurring (ranked as a low number, “1”) to least frequently occurring (ranked as a high number, "23.5”). Incident Types that had an equal number of occurrences were ranked as a "tie" and the mean of the rankings was assigned. For example, "Approaches Traffic Quickly," and "Roadway Entrance Without Clearance" occurred equally with a frequency of "6." Because their order in the ranking would consist of the ninth and tenth positions, a mean ranking of "9.5" was assigned to both Incident Types.

As can be seen from the data presented in Table 6, the most frequent Incident Type involving a LV-HV interaction was Late Braking for Stopped/Stopping Traffic. Across all 246 incidents, this particular Incident Type occurred 66 times and accounted for $26.8 \%$ of the incidents captured. The bar graph shown in Figure 7 illustrates the frequency and percentage of each Incident Type across the entire data set. As can be seen from Figure 7, the majority of the incidents (48.8\%) involved one of two different Incident Types: Late Braking for Stopped/Stopping Traffic, and Lane Change Without Sufficient Gap. 
Table 6. Frequency, Percentage, and Rank Ordering of the Incident Types Across all LVHV Incidents ( $\left.\mathbf{N}_{\text {Total }}=\mathbf{2 4 6}\right)$.

\begin{tabular}{|c|c|c|c|}
\hline Incident Type & $\begin{array}{l}\text { Frequency of all } \\
\text { LV-HV Incidents } \\
\left(\mathrm{N}_{\text {Total }}=246\right)\end{array}$ & $\begin{array}{l}\text { Percentage of } \\
\text { all LV-HV } \\
\text { Incidents } \\
\left(\mathrm{N}_{\text {Total }}=246\right)\end{array}$ & $\begin{array}{c}\text { Combined } \\
\text { Rank of all } \\
\text { LV-HV } \\
\text { Incidents }\end{array}$ \\
\hline Late Braking for Stopped/Stopping Traffic & 66 & $26.8 \%$ & 1 \\
\hline Lane Change Without Sufficient Gap & 54 & $22.0 \%$ & 2 \\
\hline Lateral Deviation of Through Vehicle & 20 & $8.1 \%$ & 3 \\
\hline Aborted Lane Change & 15 & $6.1 \%$ & 4 \\
\hline Left Turn Without Clearance & 13 & $5.3 \%$ & 5 \\
\hline Improper Passing & 12 & $4.9 \%$ & 6 \\
\hline Merge Without Sufficient Gap & 9 & $3.7 \%$ & 7 \\
\hline Conflict With Oncoming Traffic & 8 & $3.3 \%$ & 8 \\
\hline Approaches Traffic Quickly & 6 & $2.4 \%$ & 9.5 \\
\hline Roadway Entrance Without Clearance & 6 & $2.4 \%$ & 9.5 \\
\hline Following Too Closely & 5 & $2.0 \%$ & 11.5 \\
\hline Obstruction in Roadway & 5 & $2.0 \%$ & 11.5 \\
\hline Improper Lane Change & 4 & $1.6 \%$ & 13 \\
\hline Through Traffic Does Not Allow Lane Change & 3 & $1.2 \%$ & 14.5 \\
\hline Unable to Determine & 3 & $1.2 \%$ & 14.5 \\
\hline Clear Path for Emergency Vehicle & 2 & $0.8 \%$ & 18 \\
\hline Improper Stopping at an Intersection & 2 & $0.8 \%$ & 18 \\
\hline School Bus Passing Violation & 2 & $0.8 \%$ & 18 \\
\hline Through Traffic Does Not Allow Merge & 2 & $0.8 \%$ & 18 \\
\hline Wide Turn Into Adjacent Lane & 2 & $0.8 \%$ & 18 \\
\hline Backing in Roadway & 1 & $0.4 \%$ & 24 \\
\hline Improper U-Turn & 1 & $0.4 \%$ & 24 \\
\hline Improperly Covered Debris from Lead Vehicle & 1 & $0.4 \%$ & 24 \\
\hline Slow Speed & 1 & $0.4 \%$ & 24 \\
\hline Sudden Braking in Roadway & 1 & $0.4 \%$ & 24 \\
\hline Turn Without Sufficient Warning & 1 & $0.4 \%$ & 24 \\
\hline Turn/Exit From Incorrect Lane & 1 & $0.4 \%$ & 24 \\
\hline
\end{tabular}




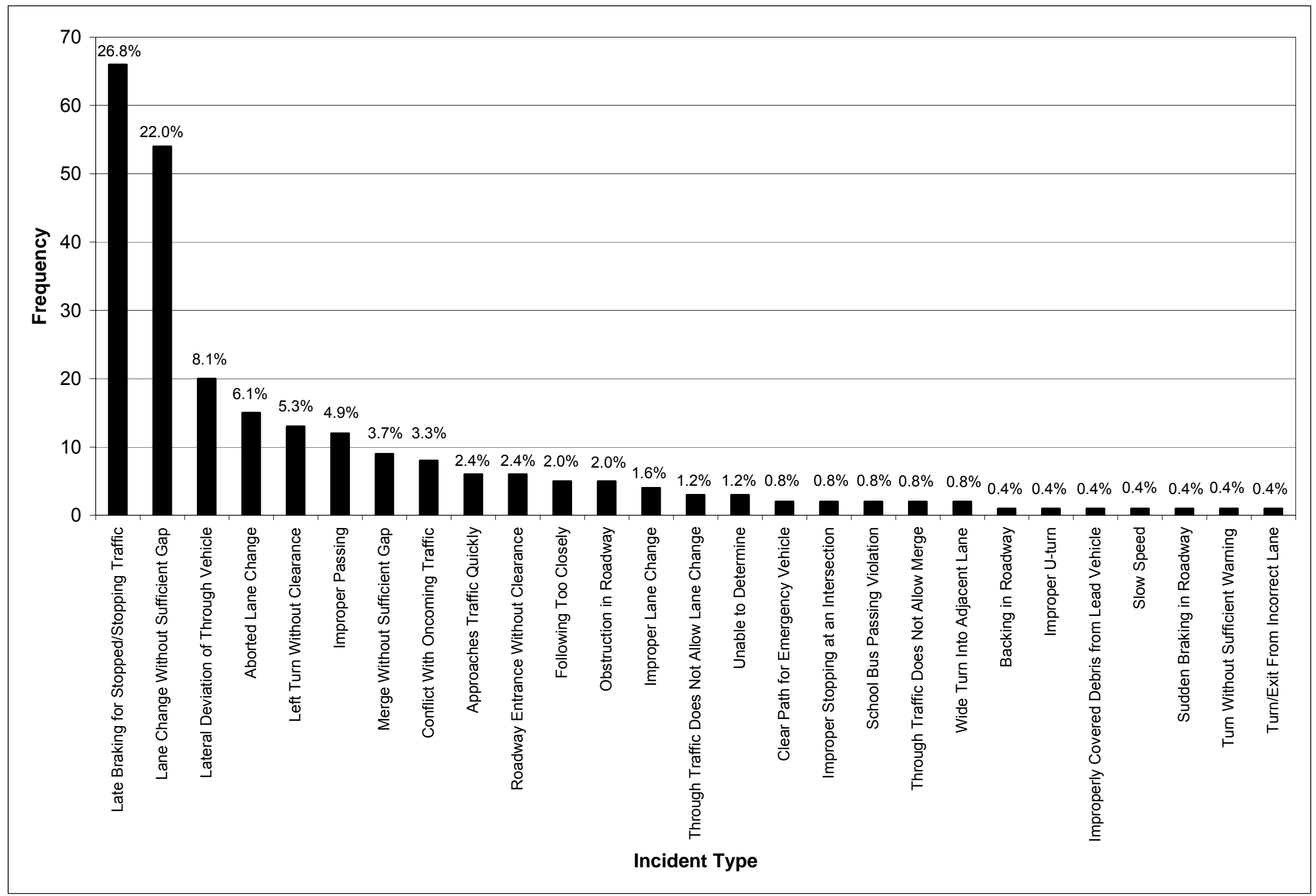

Figure 7. Frequency of Incident Types Across all LV-HV Incidents $\left(\mathrm{N}_{\text {Total }}=\mathbf{2 4 6}\right)$. 
Descriptive statistics for the Incident Types were also calculated for incidents as a function of the at-fault driver. The at-fault driver is the driver that was assessed, by the analyst, to have been responsible for causing the event. Of the $246 \mathrm{LV}-\mathrm{HV}$ interaction incidents recorded, 138 (56\%) were judged to have been the fault of the LV driver, while 79 (32\%) were attributed to the HV driver. For the remaining 29 incidents (12\%), it was unclear which vehicle driver was at-fault. By removing the "unknown" cases from the LV-HV driver at-fault analyses, it was found that the LV driver was at-fault in 64\% (138/217) of the LV-HV interaction incidents while the HV driver was at-fault in 36\% (79/217) of the incidents.

Table 7 shows the frequency, percentage, and rank ordering for the Incident Types where the HV driver was judged to be at-fault. As can be seen, the most frequent Incident Type for HV driver at-fault incidents was Lane Change Without Sufficient Gap (26.6\%), followed by Lateral Deviation of Through Vehicle (21.5\%), and Left Turn Without Clearance (13.9\%). Figure 8 shows a bar graph of the $79 \mathrm{HV}$ driver at-fault incidents as a function of the Incident Type.

Table 7. Frequency, Percentage, and Rank Ordering of the Incident Types for HV Driver At-Fault Incidents $\left(\mathbf{n}_{\mathrm{HV}}=\mathbf{7 9}\right)$.

\begin{tabular}{|c|c|c|c|}
\hline Incident Type & $\begin{array}{l}\text { Frequency of } \\
\text { HV Driver At- } \\
\text { Fault Incidents } \\
\left(\mathrm{n}_{\mathrm{HV}}=79\right)\end{array}$ & $\begin{array}{l}\text { Percentage of } \\
\text { HV Driver At- } \\
\text { Fault Incidents } \\
\left(\mathrm{n}_{\mathrm{HV}}=79\right)\end{array}$ & $\begin{array}{l}\text { Combined Rank } \\
\text { of HV Driver At- } \\
\text { Fault Incidents }\end{array}$ \\
\hline Lane Change Without Sufficient Gap & 21 & $26.6 \%$ & 1 \\
\hline Lateral Deviation of Through Vehicle & 17 & $21.5 \%$ & 2 \\
\hline Left Turn Without Clearance & 11 & $13.9 \%$ & 3 \\
\hline Aborted Lane Change & 4 & $5.1 \%$ & 4.5 \\
\hline Obstruction in Roadway & 4 & $5.1 \%$ & 4.5 \\
\hline Merge Without Sufficient Gap & 3 & $3.8 \%$ & 6.5 \\
\hline Through Traffic Does Not Allow Merge & 3 & $3.8 \%$ & 6.5 \\
\hline Roadway Entrance Without Clearance & 2 & $2.5 \%$ & 8.5 \\
\hline Wide Turn Into Adjacent Lane & 2 & $2.5 \%$ & 8.5 \\
\hline Backing in Roadway & 1 & $1.3 \%$ & 15.5 \\
\hline Clear Path for Emergency Vehicle & 1 & $1.3 \%$ & 15.5 \\
\hline Conflict With Oncoming Traffic & 1 & $1.3 \%$ & 15.5 \\
\hline Following Too Closely & 1 & $1.3 \%$ & 15.5 \\
\hline Improper Lane Change & 1 & $1.3 \%$ & 15.5 \\
\hline Improper U-Turn & 1 & $1.3 \%$ & 15.5 \\
\hline Improperly Covered Debris from Lead Vehicle & 1 & $1.3 \%$ & 15.5 \\
\hline Late Braking for Stopped/Stopping Traffic & 1 & $1.3 \%$ & 15.5 \\
\hline Slow Speed & 1 & $1.3 \%$ & 15.5 \\
\hline Sudden Braking in Roadway & 1 & $1.3 \%$ & 15.5 \\
\hline Turn Without Sufficient Warning & 1 & $1.3 \%$ & 15.5 \\
\hline Turn/Exit From Incorrect Lane & 1 & $1.3 \%$ & 15.5 \\
\hline
\end{tabular}




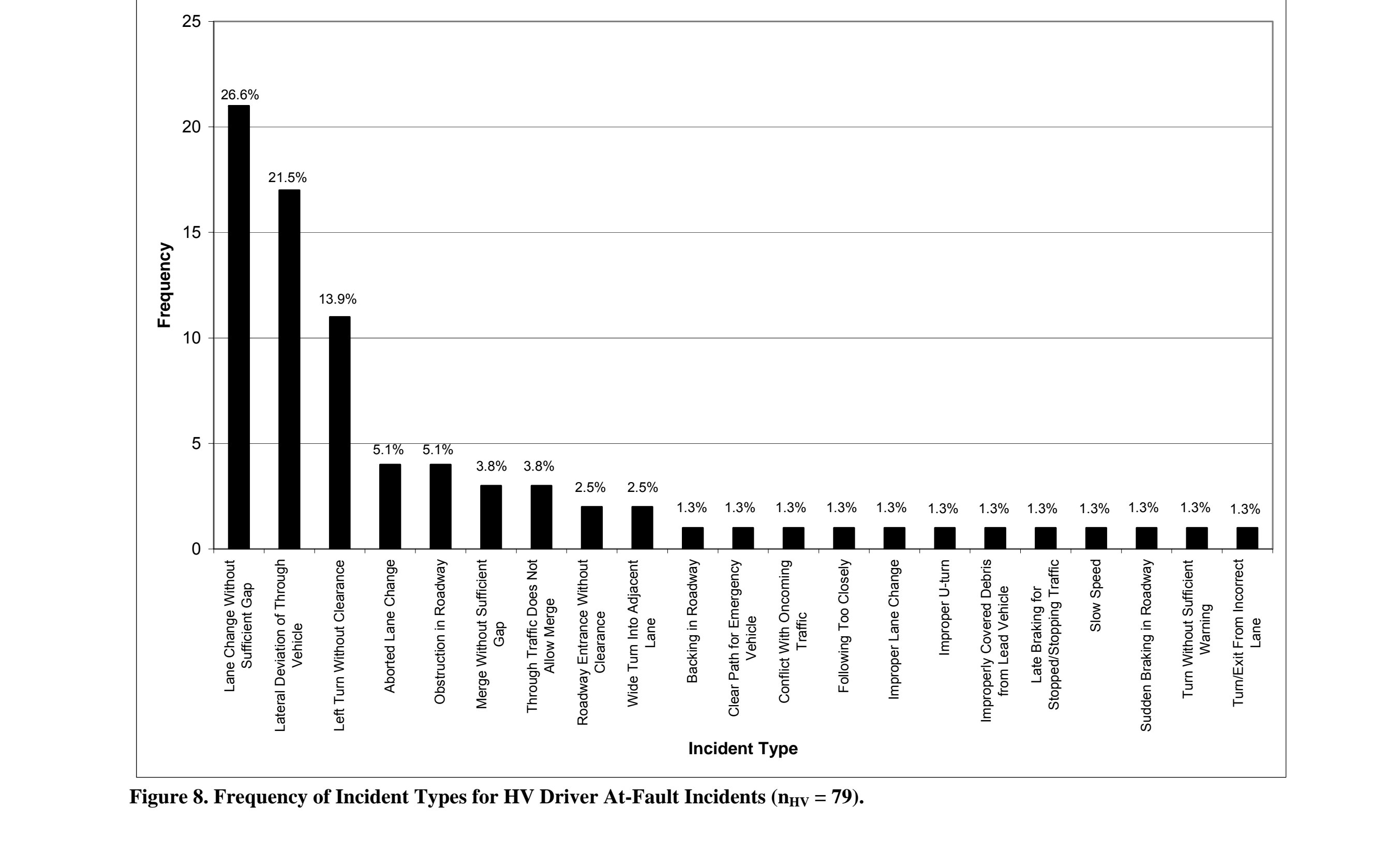


Table 8 shows the frequency, percentage, and rank ordering for the Incident Types where the LV driver was at-fault. The most frequent Incident Type for LV driver at-fault incidents was Late Braking for Stopped/Stopping Traffic (41.3\%) and Lane Change Without Sufficient Gap (21.7\%). Figure 9 shows a bar graph of the $138 \mathrm{LV}$ driver at-fault incidents as a function of the Incident Type.

Table 8. Frequency, Percentage, and Rank Ordering of the Incident Types for LV Driver At-Fault Incidents $\left(n_{L V}=138\right)$.

\begin{tabular}{|l|c|c|c|}
\hline \multicolumn{1}{|c|}{ Incident Type } & $\begin{array}{c}\text { Frequency of LV } \\
\text { Driver At-Fault } \\
\text { Incidents } \\
\left(\mathbf{n}_{\text {LV }}=138\right)\end{array}$ & $\begin{array}{c}\text { Percentage of } \\
\text { LV Driver At- } \\
\text { Fault Incidents } \\
\left(\mathbf{n}_{\text {LV }}=138\right)\end{array}$ & $\begin{array}{c}\text { Combined Rank } \\
\text { of LV Driver At- } \\
\text { Fault Incidents }\end{array}$ \\
\hline Late Braking for Stopped/Stopping Traffic & 57 & $41.3 \%$ & 1 \\
\hline Lane Change Without Sufficient Gap & 30 & $21.7 \%$ & 2 \\
\hline Aborted Lane Change & 11 & $8.0 \%$ & 3 \\
\hline Improper Passing & 10 & $7.2 \%$ & 4 \\
\hline Approaches Traffic Quickly & 6 & $4.3 \%$ & 5 \\
\hline Merge Without Sufficient Gap & 5 & $3.6 \%$ & 6 \\
\hline Following Too Closely & 4 & $2.9 \%$ & 7 \\
\hline Conflict With Oncoming Traffic & 3 & $2.2 \%$ & 9 \\
\hline Improper Lane Change & 3 & $2.2 \%$ & 9 \\
\hline Lateral Deviation of Through Vehicle & 3 & $2.2 \%$ & 9 \\
\hline Improper Stopping at an Intersection & 2 & $1.4 \%$ & 12 \\
\hline Roadway Entrance Without Clearance & 2 & $1.4 \%$ & 12 \\
\hline School Bus Passing Violation & 2 & $1.4 \%$ & 12 \\
\hline
\end{tabular}




$$
\text { Hon....... }
$$


Table 9 shows the frequency, percentage, and rank ordering for the Incident Types when the atfault driver was unknown. The most frequent Incident Type for Unknown at-fault incidents was Late Braking for Stopped/Stopping Traffic (27.6\%), followed by Conflict With Oncoming Traffic (13.8\%), Lane Change Without Sufficient Gap (10.3\%), and Unable to Determine (10.3\%). Figure 10 shows a bar graph of the 29 Unknown at-fault incidents as a function of the Incident Type.

Figure 10 illustrates the Incident Types, with respect to the driver assessed to be at-fault, by group (HV, LV, and Unknown). The figure shows that the Incident Types differed depending on whether the HV or LV driver was at-fault. Across all at-fault incidents, the most frequent Incident Type were Late Braking for Stopped/Stopping Traffic, Lane Change Without Sufficient Gap, and Lateral Deviation of Through Vehicle.

Table 9. Frequency, Percentage, and Rank Ordering of the Incident Types for Unknown At-Fault Incidents $\left(\mathbf{n}_{U n}=29\right)$.

\begin{tabular}{|l|c|c|c|}
\hline \multicolumn{1}{|c|}{ Incident Type } & $\begin{array}{c}\text { Frequency of } \\
\text { Unknown At- } \\
\text { Fault Incidents } \\
\text { (nun= 29) }\end{array}$ & $\begin{array}{c}\text { Percentage of } \\
\text { Unknown At- } \\
\text { Fault Incidents } \\
\text { (nun= 29) }\end{array}$ & $\begin{array}{c}\text { Combined Rank } \\
\text { of Unknown At- } \\
\text { Fault Incidents }\end{array}$ \\
\hline Late Braking for Stopped/Stopping Traffic & 8 & $27.6 \%$ & 1 \\
\hline Conflict With Oncoming Traffic & 4 & $13.8 \%$ & 2 \\
\hline Lane Change Without Sufficient Gap & 3 & $10.3 \%$ & 3.5 \\
\hline Unable to Determine & 3 & $10.3 \%$ & 3.5 \\
\hline Improper Passing & 2 & $6.9 \%$ & 6.5 \\
\hline Left Turn Without Clearance & 2 & $6.9 \%$ & 6.5 \\
\hline Roadway Entrance Without Clearance & 2 & $6.9 \%$ & 6.5 \\
\hline Through Traffic Does Not Allow Lane Change & 2 & $6.9 \%$ & 6.5 \\
\hline Clear Path for Emergency Vehicle & 1 & $3.4 \%$ & 10 \\
\hline Merge Without Sufficient Gap & 1 & $3.4 \%$ & 10 \\
\hline Obstruction in Roadway & 1 & $3.4 \%$ & 10 \\
\hline
\end{tabular}




$$
\text { llıı...... }
$$




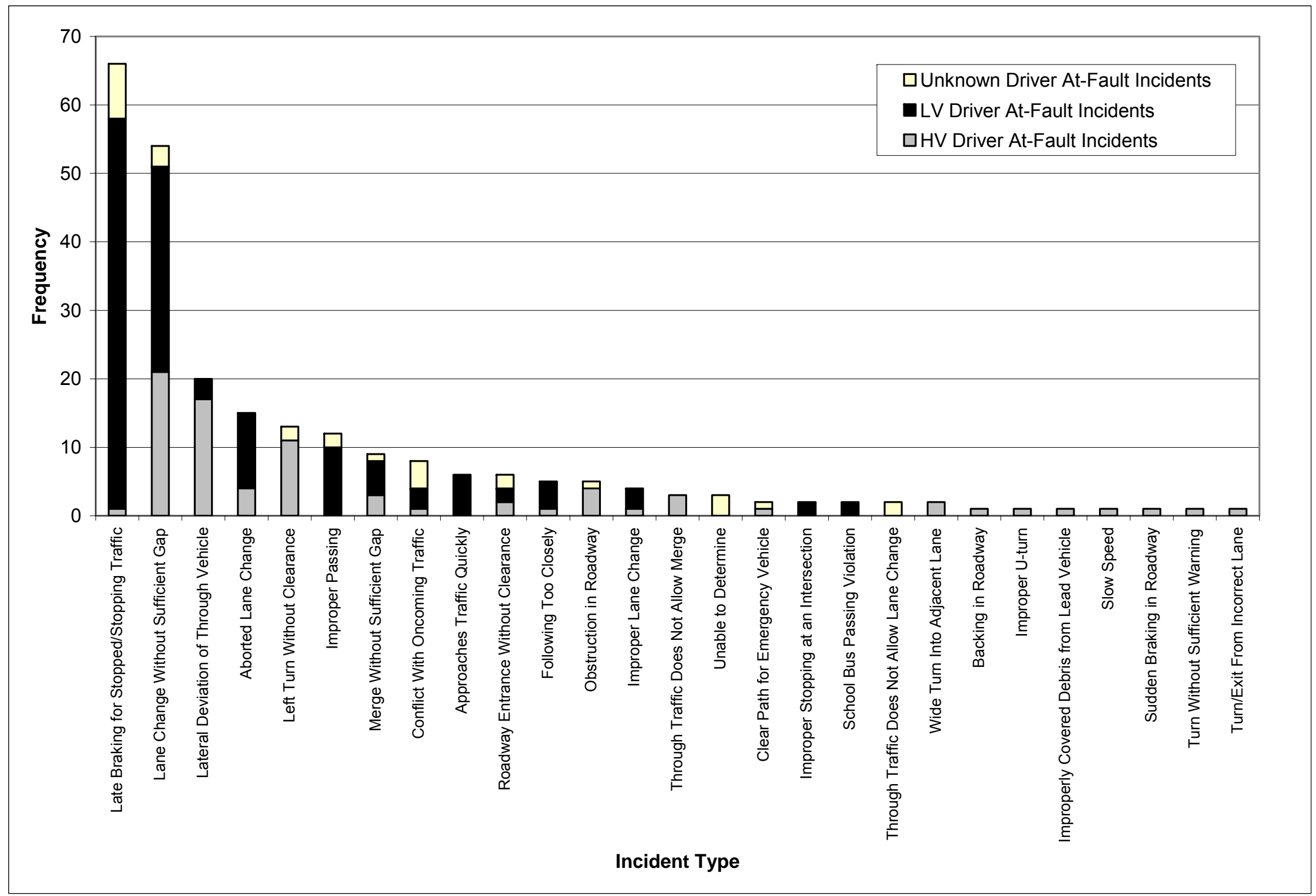

Figure 11. Frequency of Incident Types for HV, LV, and Unknown At-Fault Incidents $\left(\mathbf{n}_{\mathrm{HV}}=79, \mathbf{n}_{\mathrm{LV}}=138\right.$, and $\left.\mathbf{n}_{\mathrm{Un}}=29\right)$. 


\section{Summary of Incident Type}

Overall, the most common Incident Types were Late Braking for Stopped/Stopping Traffic (26.8\%), Lane Change Without Sufficient Gap (22\%), and Lateral Deviation of Through Vehicle (8.1\%). These three Incident Types represented 56.9\% of the LV-HV incidents.

A substantial number of $\mathrm{LV}$-HV interactions were judged to have been the fault of the LV driver. Of the $246 \mathrm{LV}-\mathrm{HV}$ interaction incidents, 56.1\% (63.6\%, excluding the incident where it was unknown if the $\mathrm{HV}$ or $\mathrm{LV}$ driver was at-fault) of the LV drivers were at-fault, 32.1\% (46.4\% excluding the Unknown at-fault incidents) of the HV drivers were at-fault, while in the

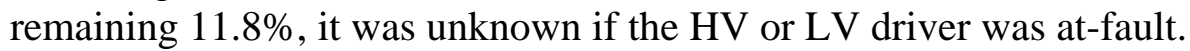

As can be seen in Figure 11, the Incident Types differed depending on whether the HV or LV driver was at-fault. The most prevalent Incident Types for HV driver at-fault incidents were Lane Change Without Sufficient Gap (26.6\%), Lateral Deviation of Through Vehicle (21.5\%), and Left Turn Without Clearance (13.9\%). These three incident types accounted for $51.7 \%$ of the HV driver at-fault incidents. The most frequent Incident Types for LV drivers were Late Braking for Stopped/Stopping Traffic (41.3\%) and Lane Change Without Sufficient Gap (21.7\%). These two incident types accounted for $63.0 \%$ of the LV driver at-fault incidents. Note that the most prevalent Incident Type for at-fault LV drivers, Lane Change Without Sufficient Gap, was similar to the truck driver focus groups' reports in Hanowski et al. (1998) who indicated that "being cut-off by LV drivers" was a frequently occurring problem in local/shorthaul trucking.

\section{Primary Maneuvers, Secondary Maneuvers and Conflict Types}

After each of the 246 incidents was classified by Incident Type, the next step in the analysis was to identify the "Primary Maneuvers" and "Secondary Maneuvers" involved in each incident. The Primary Maneuver refers to the maneuver of the driver who initiated the incident (not necessarily at-fault). Table 10 shows each Primary Maneuver and its corresponding definition. Across the 246 interaction incidents, 19 different Primary Maneuvers were identified.

\section{Table 10. List and Definition of Each Primary Maneuver Types.}

\begin{tabular}{|c|l|}
\hline Primary Maneuver & \multicolumn{1}{c|}{ Definition } \\
\hline Aborted Lane Change & $\begin{array}{l}\text { The initiating vehicle begins to make a lane change, but finds a second vehicle in its } \\
\text { blind spot and aborts the lane change. }\end{array}$ \\
\hline To the left & $\begin{array}{l}\text { The initiating vehicle begins to make a lane change to the left, but finds a second } \\
\text { vehicle in its blind spot and aborts the lane change. }\end{array}$ \\
\hline To the right & $\begin{array}{l}\text { The initiating vehicle begins to make a lane change to the right, but finds a second } \\
\text { vehicle in its blind spot and aborts the lane change. }\end{array}$ \\
\hline Avoiding Vehicle & $\begin{array}{l}\text { The initiating vehicle performs an evasive maneuver in order to avoid a second } \\
\text { vehicle. }\end{array}$ \\
\hline Swerves to the left & The initiating vehicle swerves to the left in order to avoid a second vehicle. \\
\hline Swerves to the right & The initiating vehicle swerves to the right in order to avoid a second vehicle. \\
\hline Backing & The initiating vehicle backs up in the roadway. \\
\hline
\end{tabular}




\begin{tabular}{|c|c|}
\hline Primary Maneuver & Definition \\
\hline Braking & The initiating vehicle brakes on the roadway. \\
\hline For a stop sign & The initiating vehicle brakes for a stop sign. \\
\hline For a stopped vehicle & The initiating vehicle brakes for a stopped vehicle. \\
\hline For a traffic signal & The initiating vehicle brakes for a traffic signal. \\
\hline For a yield sign & The initiating vehicle brakes for a yield sign. \\
\hline For construction & The initiating vehicle brakes for construction. \\
\hline For traffic & The initiating vehicle brakes for lead traffic. \\
\hline In a left turn lane & The initiating vehicle brakes in a left turn lane. \\
\hline In a right turn lane & The initiating vehicle brakes in a right turn lane. \\
\hline In an exit lane & The initiating vehicle brakes in an exit lane. \\
\hline Reason Unknown & The initiating vehicle brakes for an unknown reason. \\
\hline To change lanes & The initiating vehicle brakes to change lanes. \\
\hline To make a left turn & The initiating vehicle brakes to make a left turn. \\
\hline To make a right turn & The initiating vehicle brakes to make a right turn. \\
\hline Changing Lanes & The initiating vehicle changes lanes. \\
\hline To the left & The initiating vehicle changes lanes to the left. \\
\hline To the right & The initiating vehicle changes lanes to the right. \\
\hline Crossing Over Lane Line & The initiating vehicle crosses over the lane line (into another traffic lane). \\
\hline To the left & The initiating vehicle changes lanes to the left. \\
\hline To the right & The initiating vehicle changes lanes to the right. \\
\hline Enters Roadway & The initiating vehicle enters the roadway. \\
\hline From side of road & The initiating vehicle enters the roadway from the side of the road. \\
\hline From the shoulder & The initiating vehicle enters the roadway from the shoulder. \\
\hline Incomplete Lane Change & $\begin{array}{l}\text { The initiating vehicle does not complete its lane change (i.e., the vehicle is not } \\
\text { completely in the new lane and is obstructing the original lane). }\end{array}$ \\
\hline Left Turn & The initiating vehicle makes a left turn. \\
\hline Across path & The initiating vehicle makes a left turn across the path of other vehicles. \\
\hline From side road & The initiating vehicle makes a left turn from a side road. \\
\hline Oncoming traffic & The initiating vehicle makes a left turn across the path of oncoming traffic. \\
\hline Onto side road & The initiating vehicle makes a left turn onto a side road. \\
\hline Merging & The initiating vehicle merges into traffic. \\
\hline From the shoulder & The initiating vehicle merges into traffic from the shoulder. \\
\hline To the left & The initiating vehicle merges into traffic to the left. \\
\hline Move to Shoulder & The initiating vehicle moves off of the roadway onto the shoulder. \\
\hline Parked & The initiating vehicle is parked on the side of the road. \\
\hline Right turn & The initiating vehicle makes a right turn. \\
\hline From side road & The initiating vehicle makes a right turn from a side road. \\
\hline Onto side road & The initiating vehicle makes a right turn onto a side road. \\
\hline Slower Speed & The initiating vehicle is traveling at a slower speed than following traffic. \\
\hline
\end{tabular}




\begin{tabular}{|c|c|}
\hline Primary Maneuver & Definition \\
\hline Stopped & The initiating vehicle is stopped. \\
\hline At a railroad crossing & The initiating vehicle is stopped at a railroad crossing. \\
\hline At a stop sign & The initiating vehicle is stopped at a stop sign. \\
\hline At a traffic signal & The initiating vehicle is stopped at a traffic signal. \\
\hline Delivering mail & The initiating vehicle is stopped delivering mail. \\
\hline In left turn lane & The initiating vehicle is stopped in a left turn lane. \\
\hline In roadway & The initiating vehicle is stopped in the roadway. \\
\hline In traffic & The initiating vehicle is stopped in traffic. \\
\hline Loading/Unloading & The initiating vehicle is stopped loading/unloading. \\
\hline On side of road & The initiating vehicle is stopped on the side of the road. \\
\hline To make a left turn & The initiating vehicle is stopped to make a left turn. \\
\hline To make a right turn & The initiating vehicle is stopped to make a right turn. \\
\hline Drifts to the Left & The initiating vehicle drifts to the left. \\
\hline Through Traffic & The initiating vehicle is traveling straight. \\
\hline Doesn't allow merge & The initiating vehicle is traveling straight and does not allow traffic to merge. \\
\hline Oncoming traffic & The initiating vehicle is traveling straight in the opposite direction (i.e., oncoming). \\
\hline Traveling ahead & The initiating vehicle is traveling ahead of other vehicles. \\
\hline U-Turn & The initiating vehicle makes a U-turn. \\
\hline
\end{tabular}

In addition to identifying the Primary Maneuver for each incident, the Secondary Maneuver, or the maneuver of the responding driver (i.e., driver of the second vehicle involved in the interaction), was also classified. Considering the maneuvers of both vehicles involved in the incident, a clear picture of the conflict, or Conflict Type, could be determined. Table 11 shows the Conflict Types that were identified in the 246 interaction incidents that were analyzed. As can be seen, Table 11 consists of 66 different Conflict Types (i.e., Primary Maneuver and Secondary Maneuver combinations).

Table 11. The 66 Different Conflict Types Identified Across all LV-HV Incidents.

\begin{tabular}{|l|c|l|}
\hline \multirow{2}{*}{ Primary Maneuver } & Conflict Type & \\
\hline \multirow{3}{*}{ Aborted Lane Change } & 1 & Brakes and changes lanes \\
\cline { 2 - 3 } & 2 & Changes lanes \\
\cline { 2 - 3 } & 3 & No reaction \\
\cline { 2 - 3 } & 4 & Unknown if action was attempted \\
\hline \multirow{3}{*}{ Avoiding Vehicle } & 5 & No reaction \\
\hline Backing & 6 & Unknown if action was attempted \\
\hline \multirow{3}{*}{ Braking } & 7 & Backing \\
\cline { 2 - 3 } & 8 & Brakes and changes lanes \\
\cline { 2 - 3 } & 9 & Braking \\
\cline { 2 - 3 } & 10 & Changes lanes \\
\hline
\end{tabular}




\begin{tabular}{|c|c|c|}
\hline Primary Maneuver & Conflict Type & Secondary Maneuver \\
\hline \multirow{7}{*}{ Changing Lanes } & 11 & Brakes and changes lanes \\
\hline & 12 & Brakes and swerves around in lane \\
\hline & 13 & Brakes and swerves to the right/left \\
\hline & 14 & Braking \\
\hline & 15 & Continues driving \\
\hline & 16 & No reaction \\
\hline & 17 & Unknown if action was attempted \\
\hline \multirow{5}{*}{ Crossing Over Lane Line } & 18 & Brakes and changes lanes \\
\hline & 19 & Brakes and swerves to the right/left \\
\hline & 20 & Braking \\
\hline & 21 & Swerves to the right/left \\
\hline & 22 & Unknown if action was attempted \\
\hline \multirow{2}{*}{ Enters Roadway } & 23 & Brakes then passes on left \\
\hline & 24 & Braking \\
\hline \multirow{2}{*}{ Incomplete Lane Change } & 25 & Brakes and swerves right/left \\
\hline & 26 & Braking \\
\hline \multirow{6}{*}{ Left Turn } & 27 & Accelerates and honks horn \\
\hline & 28 & Accelerates and swerves right/left \\
\hline & 29 & Brakes and swerves right/left \\
\hline & 30 & Braking \\
\hline & 31 & Changes lanes \\
\hline & 32 & Stops on roadway \\
\hline \multirow{2}{*}{ Merging } & 33 & Braking \\
\hline & 34 & No reaction \\
\hline \multirow{2}{*}{ Move to Shoulder } & 35 & Brakes and swerves right/left \\
\hline & 36 & Braking \\
\hline Parked & 37 & Brakes and swerves right/left \\
\hline \multirow{4}{*}{ Right Turn } & 38 & Brakes and changes lanes \\
\hline & 39 & Brakes and swerves right/left \\
\hline & 40 & Braking \\
\hline & 41 & Stops on roadway \\
\hline \multirow{6}{*}{ Slower Speed } & 42 & Accelerates and changes lanes \\
\hline & 43 & Brakes and changes lanes \\
\hline & 44 & Brakes and passes vehicle \\
\hline & 45 & Braking \\
\hline & 46 & Changes lanes \\
\hline & 47 & Swerves with intent to change lanes \\
\hline Stopped & 48 & Brakes and changes lanes \\
\hline
\end{tabular}




\begin{tabular}{|c|c|c|}
\hline Primary Maneuver & Conflict Type & Secondary Maneuver \\
\hline & 49 & Brakes and passes vehicle \\
\hline & 50 & Brakes and swerves right/left \\
\hline & 51 & Braking \\
\hline & 52 & Changes lanes \\
\hline & 53 & No reaction \\
\hline & 54 & Passes vehicle \\
\hline & 55 & Stops on roadway \\
\hline & 56 & Swerves on roadway \\
\hline & 57 & Swerves to the right/left \\
\hline Drifts to the Left & 58 & Braking \\
\hline \multirow{6}{*}{ Through Traffic } & 59 & Brakes and changes lanes once other vehicle passes \\
\hline & 60 & Brakes and moves to the shoulder \\
\hline & 61 & Brakes and swerves to the right/left \\
\hline & 62 & Braking \\
\hline & 63 & Stops on roadway \\
\hline & 64 & Swerves to the right/left \\
\hline Traveling Ahead & 65 & Accelerates \\
\hline U-Turn & 66 & Braking \\
\hline
\end{tabular}

Table 12 shows the frequency, percentage, and rank ordering of each Primary Maneuver across the entire LV-HV 100-Car data set. The most frequent Primary Maneuver was Braking (22.8\%), followed by Changing Lanes (21.1\%) and Stopped (15\%). Figure 12 shows a bar graph of the 246 incidents as a function of the Primary Maneuver.

Table 12. Frequency, Percentage, and Rank ordering of the Primary Maneuvers Across all LV-HV Incidents $\left(\mathbf{N}_{\text {Total }}=\mathbf{2 4 6}\right)$.

\begin{tabular}{|l|c|c|c|}
\hline \multicolumn{1}{|c|}{ Primary Maneuver } & $\begin{array}{c}\text { Frequency of all } \\
\text { LV-HV Incidents } \\
\text { (N)tal }=\mathbf{2 4 6})\end{array}$ & $\begin{array}{c}\text { Percentage of all } \\
\text { LV-HV Incidents } \\
\text { ( } \mathbf{N}_{\text {Total }} \mathbf{2 4 6 )}\end{array}$ & $\begin{array}{c}\text { Combined Rank } \\
\text { of all LV-HV } \\
\text { Incidents }\end{array}$ \\
\hline Braking & 56 & $22.8 \%$ & 1 \\
\hline Changing Lanes & 52 & $21.1 \%$ & 2 \\
\hline Stopped & 37 & $15.0 \%$ & 3 \\
\hline Crossing Over Lane Line & 19 & $7.7 \%$ & 4 \\
\hline Left Turn & 16 & $6.5 \%$ & 5.5 \\
\hline Through Traffic & 16 & $6.5 \%$ & 5.5 \\
\hline Slower Speed & 15 & $6.1 \%$ & 6 \\
\hline Aborted Lane Change & 8 & $3.3 \%$ & 7 \\
\hline Merging & 6 & $2.4 \%$ & 9.5 \\
\hline Right Turn & 6 & $2.4 \%$ & 9.5 \\
\hline
\end{tabular}




\begin{tabular}{|c|c|c|c|}
\hline Primary Maneuver & $\begin{array}{l}\text { Frequency of all } \\
\text { LV-HV Incidents } \\
\left(\mathrm{N}_{\text {Total }}=246\right)\end{array}$ & $\begin{array}{l}\text { Percentage of all } \\
\text { LV-HV Incidents } \\
\left(\mathrm{N}_{\text {Total }}=246\right)\end{array}$ & $\begin{array}{l}\text { Combined Rank } \\
\text { of all LV-HV } \\
\text { Incidents }\end{array}$ \\
\hline Avoiding Vehicle & 3 & $1.2 \%$ & 11.5 \\
\hline Move to Shoulder & 3 & $1.2 \%$ & 11.5 \\
\hline Enters Roadway & 2 & $0.8 \%$ & 13.5 \\
\hline Incomplete Lane Change & 2 & $0.8 \%$ & 13.5 \\
\hline Backing & 1 & $0.4 \%$ & 17 \\
\hline Drifts to the Left & 1 & $0.4 \%$ & 17 \\
\hline Parked & 1 & $0.4 \%$ & 17 \\
\hline Traveling Ahead & 1 & $0.4 \%$ & 17 \\
\hline U-Turn & 1 & $0.4 \%$ & 17 \\
\hline
\end{tabular}




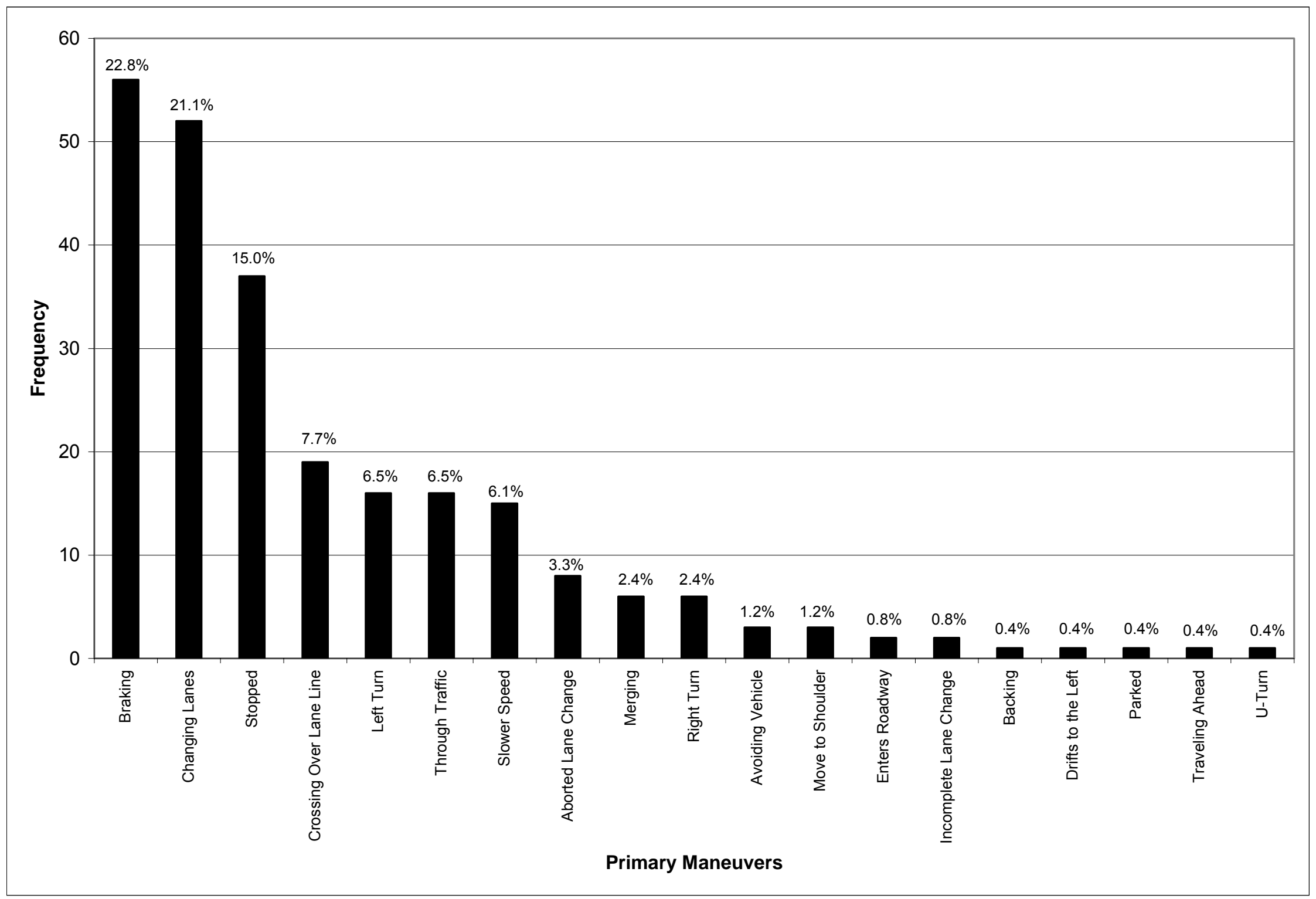

Figure 12. Frequency of Primary Maneuvers Across all LV-HV Incidents $\left(\mathrm{N}_{\text {Total }}=\mathbf{2 4 6}\right)$. 
Table 13 displays the frequency, percentage, and rank ordering for the Primary Maneuvers where the HV driver was at-fault. As can be seen in Table 13, the most frequent Primary Maneuver for HV driver at-fault incidents was Changing Lanes (32.9\%), followed by Crossing Over Lane Line (20.3\%) and Left Turn (15.2\%). Figure 13 shows a bar graph of the $79 \mathrm{HV}$ driver at-fault incidents as a function of the Primary Maneuver.

Table 13. Frequency, Percentage, and Rank Ordering of the Primary Maneuvers for the HV Driver At-Fault Incidents $\left(\mathbf{n}_{\mathrm{HV}}=\mathbf{7 9}\right)$.

\begin{tabular}{|l|c|c|c|}
\hline \multicolumn{1}{|c|}{ Primary Maneuver } & $\begin{array}{c}\text { Frequency of HV } \\
\text { Driver At-Fault } \\
\text { Incidents } \\
\left.\mathbf{n}_{\text {HV }} \mathbf{7 9}\right)\end{array}$ & $\begin{array}{c}\text { Percentage of } \\
\text { HV Driver At- } \\
\text { Fault Incidents } \\
\left.\mathbf{( n}_{\text {HV }}=\mathbf{7 9}\right)\end{array}$ & $\begin{array}{c}\text { Combined Rank } \\
\text { of HV Driver At- } \\
\text { Fault Incidents }\end{array}$ \\
\hline Changing Lanes & 26 & $32.9 \%$ & 1 \\
\hline Crossing Over Lane Line & 16 & $20.3 \%$ & 2 \\
\hline Left Turn & 12 & $15.2 \%$ & 3 \\
\hline Stopped & 4 & $5.1 \%$ & 4.5 \\
\hline Through Traffic & 4 & $5.1 \%$ & 4.5 \\
\hline Braking & 3 & $3.8 \%$ & 6.5 \\
\hline Merging & 3 & $3.8 \%$ & 6.5 \\
\hline Right Turn & 2 & $2.5 \%$ & 8 \\
\hline Aborted Lane Change & 1 & $1.3 \%$ & 13 \\
\hline Backing & 1 & $1.3 \%$ & 13 \\
\hline Enters Roadway & 1 & $1.3 \%$ & 13 \\
\hline Incomplete Lane Change & 1 & $1.3 \%$ & 13 \\
\hline Moved to Shoulder & 1 & $1.3 \%$ & 13 \\
\hline Parked & 1 & $1.3 \%$ & 13 \\
\hline Slower Speed & 1 & $1.3 \%$ & 13 \\
\hline Traveling Ahead & 1 & $1.3 \%$ & 13 \\
\hline U-Turn & 1 & $1.3 \%$ & 13 \\
\hline
\end{tabular}




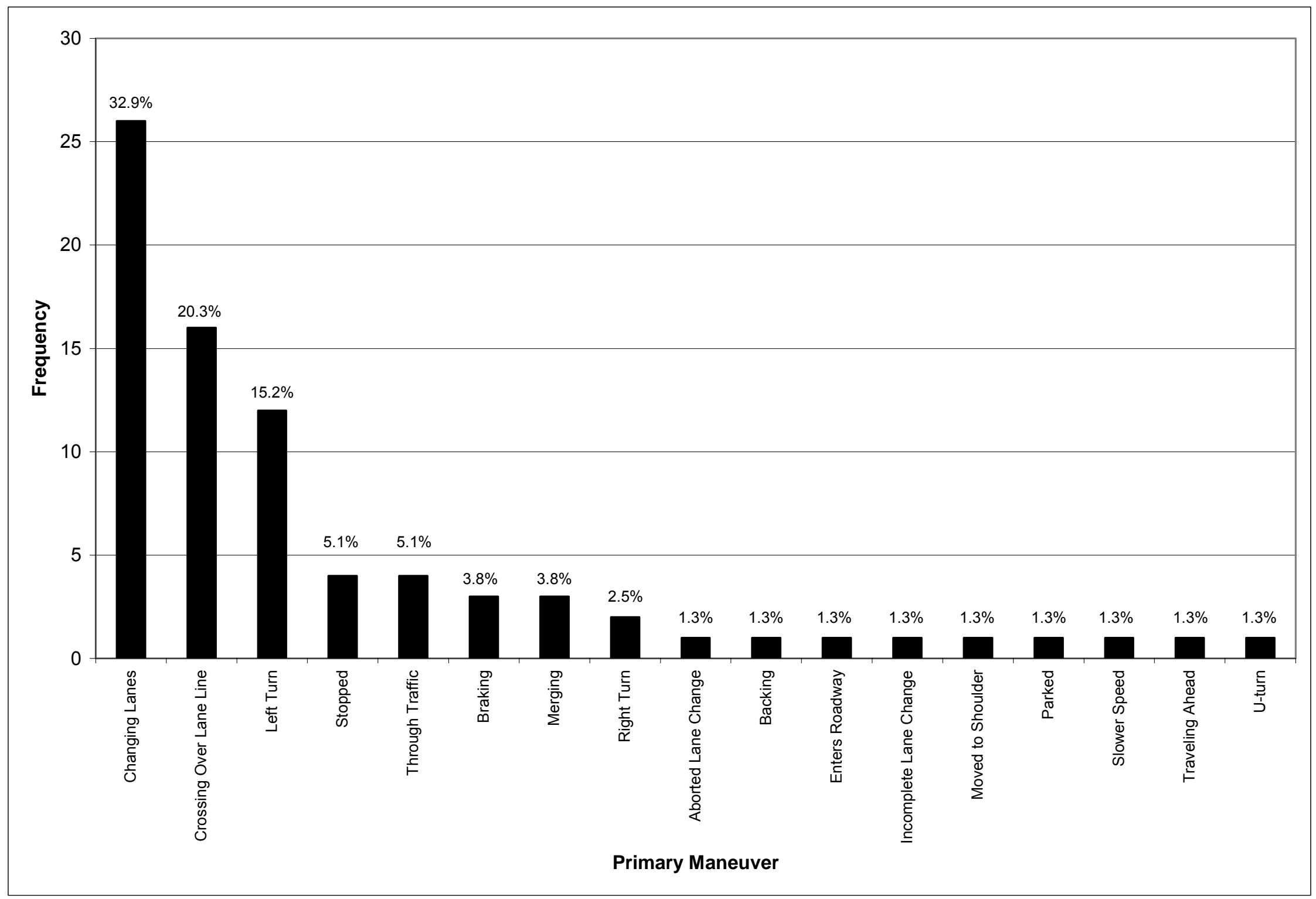

Figure 13. Frequency of Primary Maneuvers for HV Driver At-Fault Incidents $\left(\mathbf{n}_{\mathrm{HV}}=79\right)$. 
Table 14 displays the frequency, percentage, and rank ordering for the Primary Maneuvers where the LV driver was at-fault. As can be seen in Table 14, the most frequent Primary Maneuver for LV driver at-fault incidents was Braking (32.6\%), followed by Stopped (21.7\%) and Changing Lanes (16.7\%). Figure 14 shows a bar graph of the $138 \mathrm{LV}$ driver at-fault incidents as a function of the Primary Maneuver.

Table 14. Frequency, Percentage, and Rank Ordering of the Primary Maneuvers for the LV Driver At-Fault Incidents $\left(\mathbf{n}_{\mathrm{LV}}=138\right)$.

\begin{tabular}{|l|c|c|c|}
\hline \multicolumn{1}{|c|}{ Primary Maneuver } & $\begin{array}{c}\text { Frequency of LV } \\
\text { Driver At-Fault } \\
\text { Incidents } \\
\left(\mathbf{n}_{\mathbf{L V}}=\mathbf{1 3 8}\right)\end{array}$ & $\begin{array}{c}\text { Percentage of } \\
\text { LV Driver At- } \\
\text { Fault Incidents } \\
\left(\mathbf{n}_{\mathbf{L V}}=\mathbf{1 3 8}\right)\end{array}$ & $\begin{array}{c}\text { Combined Rank } \\
\text { of LV Driver At- } \\
\text { Fault Incidents }\end{array}$ \\
\hline Braking & 45 & $32.6 \%$ & 1 \\
\hline Stopped & 30 & $21.7 \%$ & 2 \\
\hline Changing Lanes & 23 & $16.7 \%$ & 3 \\
\hline Slower Speed & 13 & $9.4 \%$ & 4 \\
\hline Aborted Lane Change & 7 & $5.1 \%$ & 5 \\
\hline Through Traffic & 6 & $4.3 \%$ & 6 \\
\hline Avoiding Vehicle & 3 & $2.2 \%$ & 8 \\
\hline Merging & 3 & $2.2 \%$ & 8 \\
\hline Right Turn & 3 & $2.2 \%$ & 10 \\
\hline Crossing Over Lane Line & 2 & $1.4 \%$ & 12 \\
\hline Drifts to the Left & 1 & $0.7 \%$ & 12 \\
\hline Left Turn & 1 & $0.7 \%$ & 12 \\
\hline Moved to Shoulder & 1 & $0.7 \%$ & \\
\hline
\end{tabular}




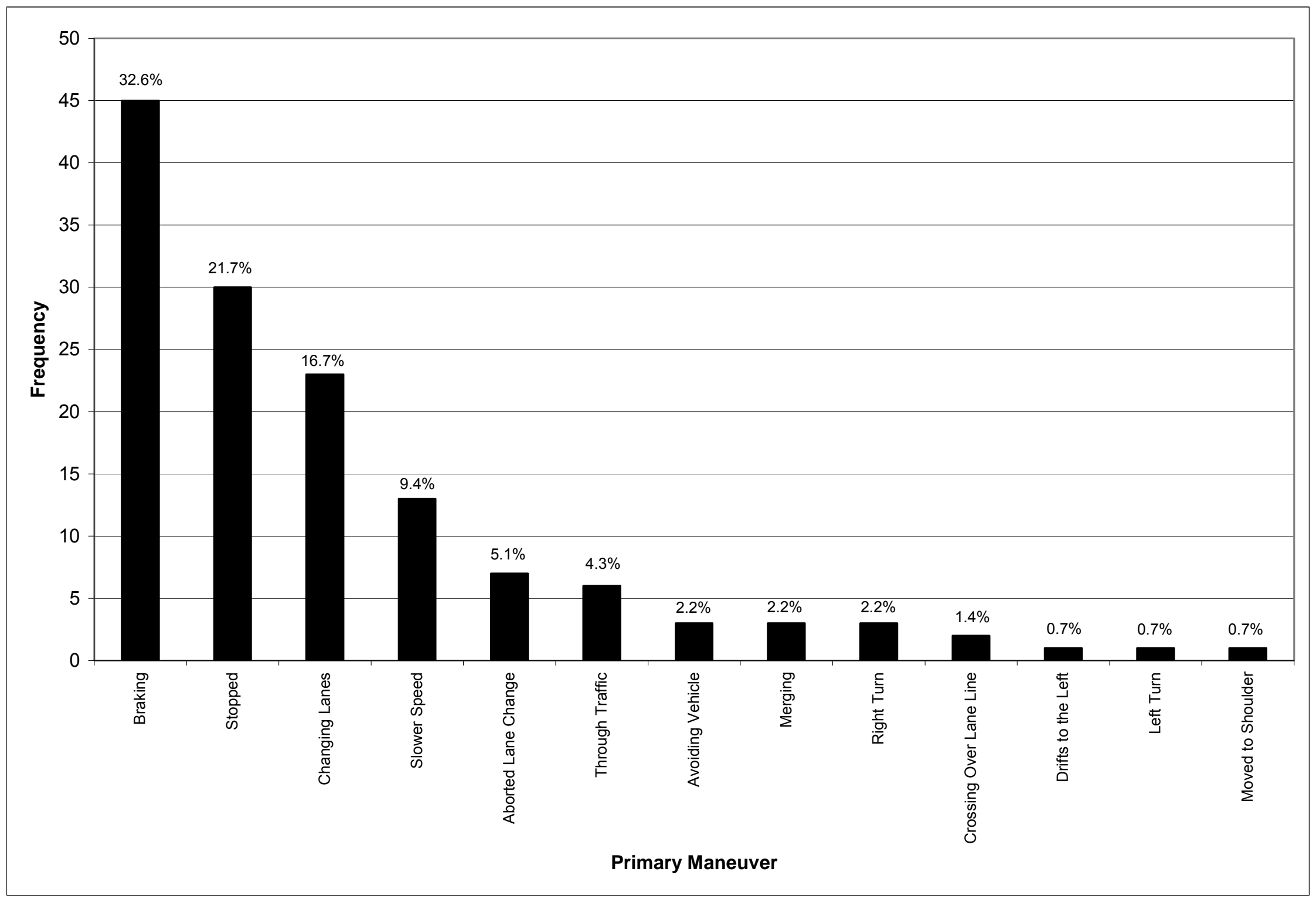

Figure 14. Frequency of Primary Maneuvers for LV Driver At-Fault Incidents $\left(n_{L V}=138\right)$. 
Table 15 displays the frequency, percentage, and rank ordering for the Primary Maneuvers where it was unknown if the HV or LV driver was at-fault. As can be seen in Table 15, the most frequent Primary Maneuver for Unknown at-fault incidents was Braking (27.6\%), followed by Through Traffic (20.7\%), Changing Lanes (10.3\%), Left Turn (10.3\%), and Stopped (10.3\%). Figure 15 shows a bar graph of the 29 Unknown at-fault incidents as a function of the Primary Maneuver.

Figure 16 illustrates the Primary Maneuvers, with respect to the driver assessed to have been atfault, by group (LV, HV, and Unknown). Figure 16 illustrates that the Primary Maneuvers differ depending on whether the $\mathrm{HV}$ or $\mathrm{LV}$ driver was at-fault. Across all incidents, the most frequent Primary Maneuvers were Braking, Changing Lanes, and Stopped.

Table 15. Frequency, Percentage, and Rank Ordering of the Primary Maneuvers for the Unknown At-Fault Incidents $\left(\mathbf{n}_{\mathrm{Un}}=29\right)$.

\begin{tabular}{|l|c|c|c|}
\hline \multicolumn{1}{|c|}{ Primary Maneuver } & $\begin{array}{c}\text { Frequency of } \\
\text { Unknown At-Fault } \\
\text { Incidents } \\
\text { (Nun } \mathbf{2 9 )}\end{array}$ & $\begin{array}{c}\text { Percentage of } \\
\text { Unknown At-Fault } \\
\text { Incidents } \\
\left.\text { ( } \text { (Un }_{\text {Un }} \mathbf{2 9}\right)\end{array}$ & $\begin{array}{c}\text { Combined Rank of } \\
\text { Unknown At-Fault } \\
\text { Incidents }\end{array}$ \\
\hline Braking & 8 & $27.6 \%$ & 1 \\
\hline Through Traffic & 6 & $20.7 \%$ & 2 \\
\hline Changing Lanes & 3 & $10.3 \%$ & 4 \\
\hline Left Turn & 3 & $10.3 \%$ & 4 \\
\hline Stopped & 3 & $10.3 \%$ & 4 \\
\hline Crossing Over Lane Line & 1 & $3.4 \%$ & 8.5 \\
\hline Enters Roadway & 1 & $3.4 \%$ & 8.5 \\
\hline Incomplete Lane Change & 1 & $3.4 \%$ & 8.5 \\
\hline Moved to Shoulder & 1 & $3.4 \%$ & 8.5 \\
\hline Right Turn & 1 & $3.4 \%$ & 8.5 \\
\hline Slower Speed & 1 & $3.4 \%$ & 8.5 \\
\hline
\end{tabular}




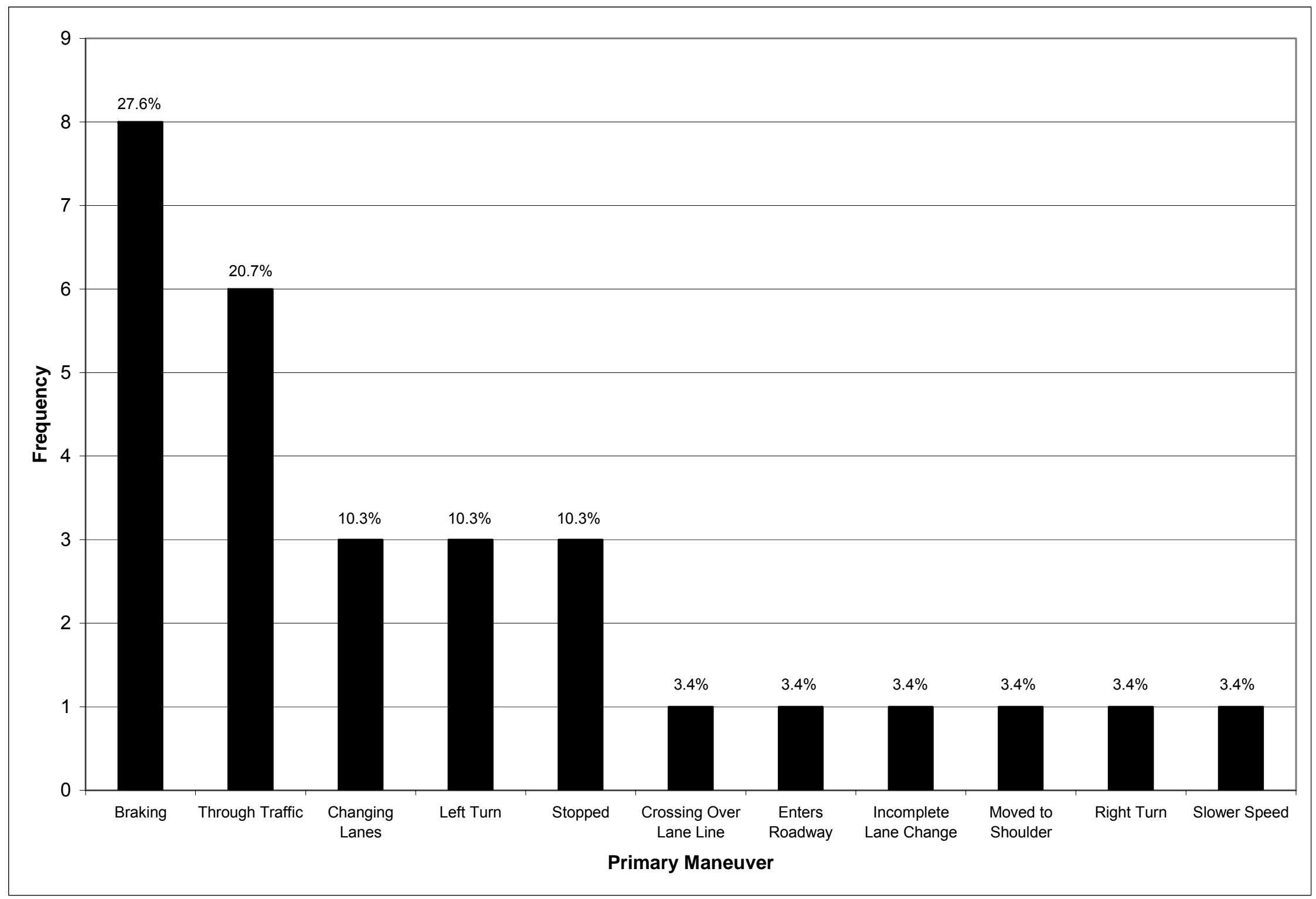

Figure 15. Frequency of Primary Maneuvers for Unknown At-Fault Incidents $\left(n_{U n}=29\right)$. 


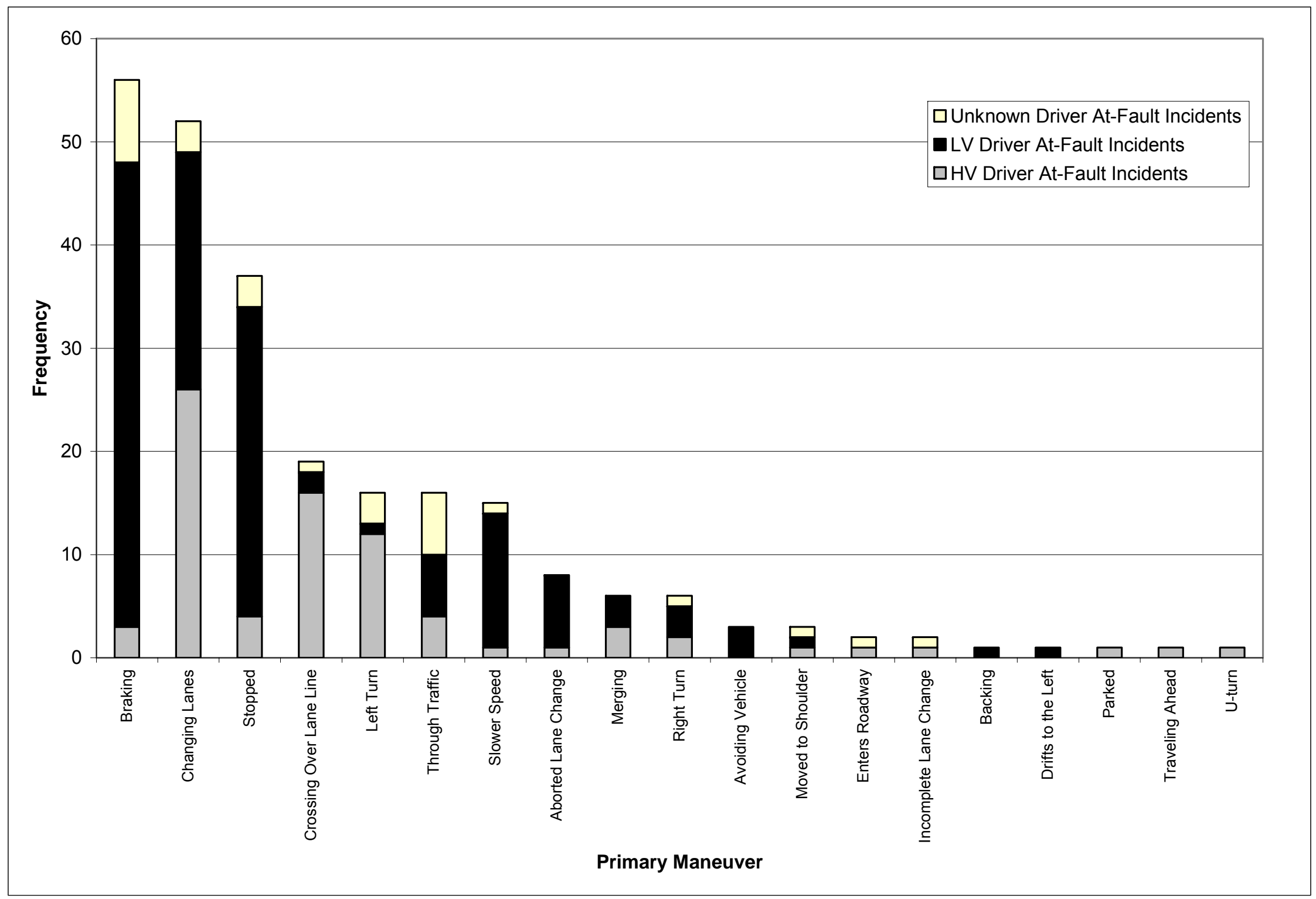

Figure 16. Frequency of Primary Maneuvers for HV, LV, and Unknown At-Fault Incidents $\left(\mathbf{n}_{\mathrm{HV}}=\mathbf{7 9}, \mathbf{n}_{\mathrm{LV}}=138\right.$, and $\left.\mathbf{n}_{U n}=29\right)$. 


\section{$\underline{\text { Summary of Primary Maneuvers }}$}

Overall, the most frequent Primary Maneuvers were Braking (22.7\%), Changing Lanes (21.1\%), and Stopped (15\%). These three Primary Maneuvers represented 58.9\% of the recorded incidents. The most frequent Primary Maneuvers, excluding the Unknown at-fault incidents, were Braking (25.8\%), Changing Lanes (21.1\%), and Stopped (15\%). These three maneuver types represented $60.4 \%$ of the Primary Maneuvers for both HV and LV driver at-fault incidents.

As can be seen in Figure 16, the Primary Maneuvers differed depending on whether the HV or LV driver was at-fault. The most prevalent Primary Maneuvers for HV driver at-fault incidents were Changing Lanes (32.9\%), Crossing Over Lane Line (20.3\%), and Left Turn (15.2\%). These three Primary Maneuvers accounted for $68.4 \%$ of the HV driver at-fault incidents. The most prevalent Primary Maneuvers for LV driver at-fault incidents were Braking (32.6\%), Stopped (21.7\%), and Changing Lanes (16.7\%). These three Primary Maneuvers accounted for $71 \%$ of the LV driver at-fault incidents. From this data it appears that most Primary Maneuvers for $\mathrm{HV}$ driver at-fault incidents occurred when the HV was in-motion and changing lanes or crossing the lane line. In contrast, a significant proportion of the Primary Maneuvers when the $\mathrm{LV}$ driver was at-fault occurred when the $\mathrm{LV}$ was decelerating or stopped.

\section{Contributing Factors}

Just as the Incident Types describe the action or what happened during an incident, Contributing Factors provide likely reasons why an incident occurred. For each incident that was analyzed, a number of Contributing Factors were identified. It should be noted that the Contributing Factor categories were taken from Wierwille, Kieliszewski, Hanowski, Keisler, and Olsen (2000) and from the GES Physical Impairments screen (USDOT/NHTSA, 2003, p. 434). Each Contributing Factor and its corresponding definition are shown in Table 16. It should be noted that an incident could receive multiple Contributing Factors (e.g., both Driver Impairment and Willful Behavior). In addition, the Contributing Factor was based solely on the behaviors of the LV driver. Without cameras inside the HV there was no way to determine, with any degree of certainty, the behavior(s) of the HV driver.

Even when the HV driver was judged to have been at-fault, the behaviors of the LV driver are identified. Put another way, for the events that were caused by the LV driver, the analyses considered the LV driver's behaviors that may have contributed to the event. For events where the HV-driver was at-fault, the analysis also considered the LV driver's behaviors. However, the consideration is for the LV driver behaviors that occurred as the driver reacted to the HV driver's actions. For example, an HV driver at-fault incident that was assigned a Contributing Factors of "Distraction" would refer to the LV driver being distracted (not the HV driver) as he/she reacted to the event. Table 16 shows each of the contributing factors identified in this study. A complete list of contributing factors can be found in Dingus et al. (2004). 
Table 16. Contributing Factors Used to Identify the Primary Cause of the Incident.

\begin{tabular}{|c|c|}
\hline Contributing Factor & Description \\
\hline \multicolumn{2}{|c|}{ Driver Impairment - The driver's behavior, judgment or driving ability is altered or hindered. } \\
\hline Drowsy, fatigued & $\begin{array}{l}\text { Impairs the driver's attention and/or judgment. Determined through yawning, } \\
\text { slow eye closures, heavy eyes, etc. }\end{array}$ \\
\hline Angry & $\begin{array}{l}\text { Impairs the driver's attention and/or judgment. Determined through facial } \\
\text { expressions or verbal comments made by the driver. }\end{array}$ \\
\hline Other emotional state & $\begin{array}{l}\text { Impairs the driver's attention and/or judgment. Determined through facial } \\
\text { expressions or verbal comments made by the driver. }\end{array}$ \\
\hline Distracted & Impairs the driver's attention and/or judgment. \\
\hline Other & Impairs the driver's ability to drive safely. \\
\hline Unknown & Impairs the driver's ability to drive safely. \\
\hline \multicolumn{2}{|c|}{ Willful Behavior - The driver knowingly and purposefully drives in an unsafe or inappropriate manner } \\
\hline Aggressive driving & $\begin{array}{l}\text { The driver intentionally drives unsafely or inappropriately, usually due to } \\
\text { impatience (e.g., quickly passes another vehicle just before a turn, fails to } \\
\text { remain a safe distance from another vehicle, excessive speed). }\end{array}$ \\
\hline $\begin{array}{l}\text { Purposeful violation of traffic } \\
\text { laws }\end{array}$ & $\begin{array}{l}\text { The driver knowingly violates a traffic law (e.g., fails to respond to a traffic } \\
\text { signal or stop sign). }\end{array}$ \\
\hline \multicolumn{2}{|c|}{ Driver Proficiency - An individual's driving skills, abilities, or knowledge is inadequate } \\
\hline Driving techniques & $\begin{array}{l}\text { Driver appears unsure or incompetent as to how to safely perform a driving } \\
\text { maneuver (e.g., failing to check for traffic before proceeding onto a roadway). }\end{array}$ \\
\hline \multicolumn{2}{|c|}{$\begin{array}{l}\text { Infrastructure - The driver's physical surroundings hinder his/her ability to drive safely and } \\
\text { appropriately or confuse the driver. }\end{array}$} \\
\hline Roadway alignment & $\begin{array}{l}\text { It is difficult for the driver to maneuver due to the geometry of a roadway or } \\
\text { intersection (e.g., an arrow roadway or sharp turn). }\end{array}$ \\
\hline Roadway delineation & Poor visibility (e.g., faded paint) or positioning of roadway or lane borders. \\
\hline
\end{tabular}

Table 17 shows the frequency, percentage, and rank ordering for each Contributing Factor across all 246 incidents. As can be seen in Table 17, the most frequent Contributing Factor was Driving Techniques (49.5\%), followed by Unknown (24\%), Distracted (18.7\%), and Aggressive Driving $(15 \%)^{3}$. Figure 17 shows a bar graph of the 246 incidents as a function of the Contributing Factor.

\footnotetext{
${ }^{3}$ Since more than one Contributing Factor could be coded with each incident, the percentages total more than $100 \%$. This procedure was also used by Blower (1998). The denominator for determining the percentages was the total number of LV-HV interactions for that data set (Example: The denominators in Tables 15 and 16 were 246 and 79 , respectively).
} 
Table 17. Frequency, Percentage, and Rank Ordering of the Contributing Factors Across all LV-HV Incidents $\left(\mathbf{N}_{\text {Total }}=\mathbf{2 4 6}\right)$.

\begin{tabular}{|l|c|c|c|}
\hline \multicolumn{1}{|c|}{ Contributing Factors } & $\begin{array}{c}\text { Frequency of } \\
\text { all LV-HV } \\
\text { Incidents }\end{array}$ & $\begin{array}{c}\text { Percentage of } \\
\text { all LV-HV } \\
\text { Incidents }\end{array}$ & $\begin{array}{c}\text { Combined } \\
\text { Rank of all LV- } \\
\text { HV Incidents }\end{array}$ \\
\hline Driving techniques & 122 & $49.5 \%$ & 1 \\
\hline Unknown & 59 & $24 \%$ & 2 \\
\hline Distracted & 46 & $18.7 \%$ & 3 \\
\hline Aggressive driving & 37 & $15 \%$ & 4 \\
\hline Drowsy & 9 & $3.7 \%$ & 5 \\
\hline Purposeful violation of traffic laws & 8 & $3.3 \%$ & 6.5 \\
\hline Roadway alignment & 8 & $3.3 \%$ & 6.5 \\
\hline Roadway delineation & 3 & $1.2 \%$ & 8 \\
\hline Angry & 2 & $0.8 \%$ & 9.5 \\
\hline Other emotional state & 2 & $0.8 \%$ & 9.5 \\
\hline Other & 1 & $0.4 \%$ & 11 \\
\hline
\end{tabular}




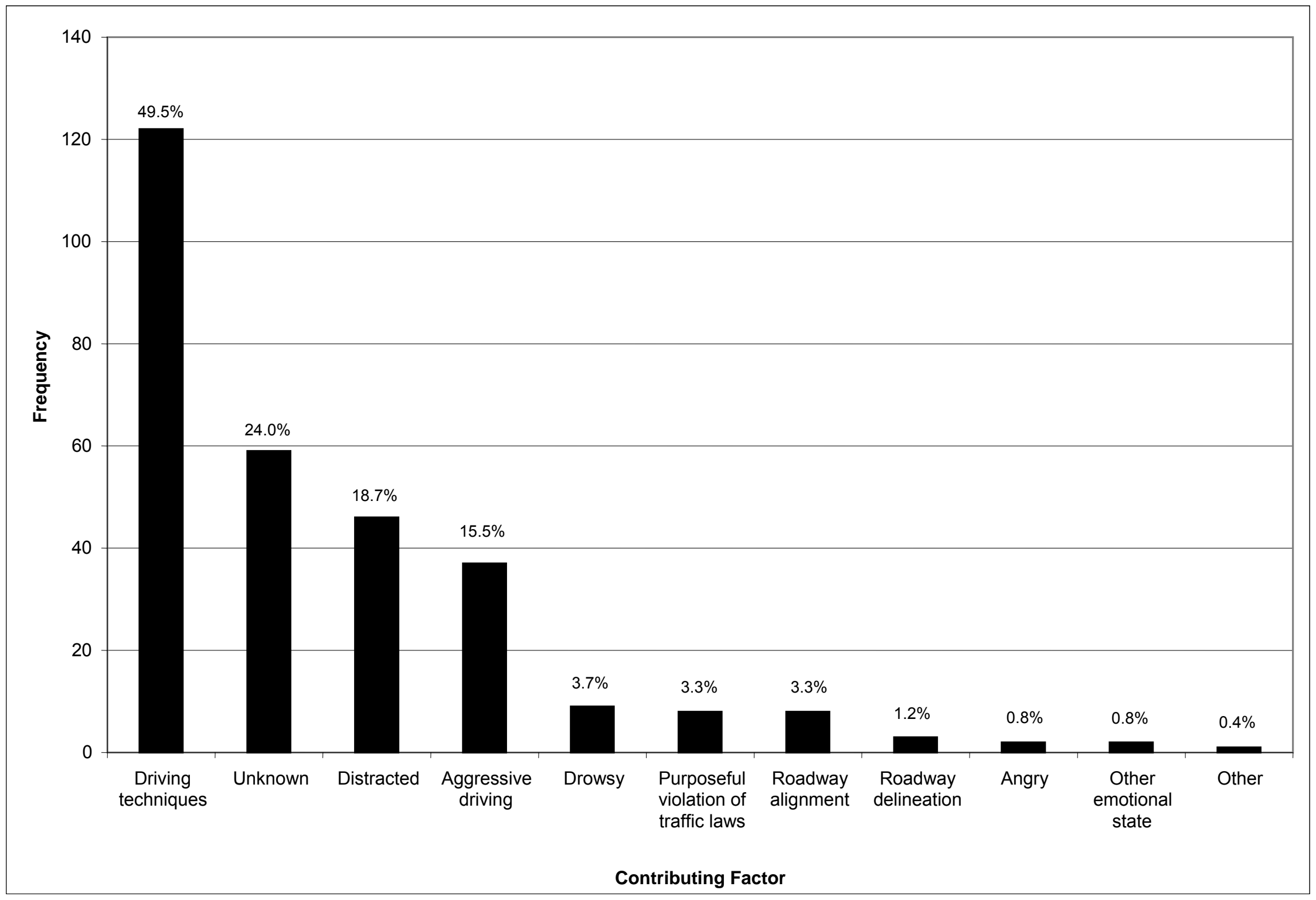

Figure 17. Frequency of Contributing Factors Across all LV-HV Incidents $\left(\mathrm{N}_{\text {Total }}=\mathbf{2 4 6 .}\right.$.) 
Table 18 shows the frequency, percentage, and rank ordering of each Contributing Factor for the HV driver at-fault incidents. As can be seen in Table 18, the most frequent Contributing Factor was Unknown (68.4\%), followed by Driving Techniques (15.2\%), and Distracted (11.4\%). Figure 18 shows a bar graph of the $79 \mathrm{HV}$ driver at-fault incidents as a function of the Contributing Factor.

Table 18. Frequency, Percentage, and Rank Ordering of the Contributing Factors for HV Drivers in the HV At-Fault Incidents $\left(\mathbf{n}_{\mathrm{HV}}=79\right)$.

\begin{tabular}{|l|c|c|c|}
\hline \multicolumn{1}{|c|}{ Contributing Factors } & $\begin{array}{c}\text { Frequency of HV } \\
\text { Driver At-Fault } \\
\text { Incidents } \\
\left(\mathbf{n}_{\text {HV }}=\mathbf{7 9}\right)\end{array}$ & $\begin{array}{c}\text { Percentage of } \\
\text { HV Driver At- } \\
\text { Fault Incidents } \\
\left(\mathbf{n}_{\text {HV }}=\mathbf{7 9}\right)\end{array}$ & $\begin{array}{c}\text { Combined Rank } \\
\text { of HV Driver At- } \\
\text { Fault Incidents }\end{array}$ \\
\hline Unknown & 54 & $68.4 \%$ & 1 \\
\hline Driving techniques & 12 & $15.2 \%$ & 2 \\
\hline Distracted & 9 & $11.4 \%$ & 3 \\
\hline Aggressive driving & 3 & $3.8 \%$ & 4 \\
\hline Drowsy & 2 & $2.5 \%$ & 5.5 \\
\hline Roadway alignment & 2 & $2.5 \%$ & 5.5 \\
\hline Angry & 1 & $1.3 \%$ & 8 \\
\hline Other emotional state & 1 & $1.3 \%$ & 8 \\
\hline Roadway delineation & 1 & $1.3 \%$ & 8 \\
\hline
\end{tabular}




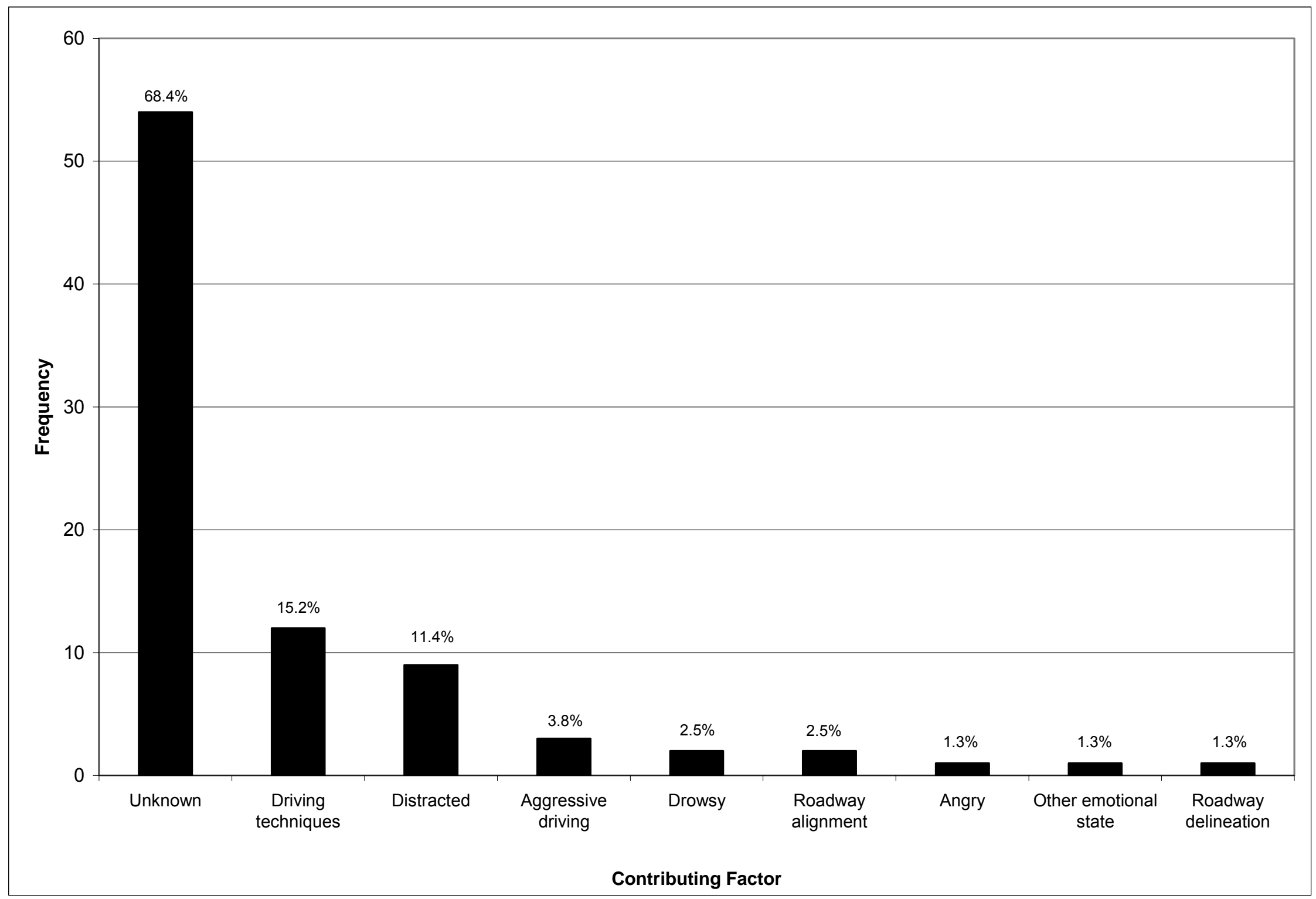

Figure 18. Frequency of Contributing Factors for LV Drivers in the HV At-Fault Incidents $\left(\mathbf{n}_{\mathrm{HV}}=\mathbf{7 9}\right)$. 
Table 19 shows the frequency, percentage, and rank ordering of each Contributing Factor for the LV driver at-fault incidents. As can be seen in Table 19, the most frequent Contributing Factor for LV driver at-fault incidents was Driving Techniques (70.3\%), followed by Distracted (22.5\%) and Aggressive Driving (22.5\%). Figure 19 shows a bar graph of the 138 LV driver atfault incidents as a function of the Contributing Factor.

Table 19. Frequency, Percentage, and Rank Ordering of the Contributing Factors for the LV Driver At-Fault Incidents $\left(\mathbf{n}_{\mathrm{LV}}=\mathbf{1 3 8}\right)$.

\begin{tabular}{|l|c|c|c|}
\hline \multicolumn{1}{|c|}{ Contributing Factors } & $\begin{array}{c}\text { Frequency of LV } \\
\text { Driver At-Fault } \\
\text { Incidents } \\
\left(\mathbf{n}_{\text {LV }}=\mathbf{1 3 8}\right)\end{array}$ & $\begin{array}{c}\text { Percentage of } \\
\text { LV Driver At- } \\
\text { Fault Incidents } \\
\left(\mathbf{n}_{\text {LV }}=138\right)\end{array}$ & $\begin{array}{c}\text { Combined Rank } \\
\text { of LV Driver At- } \\
\text { Fault Incidents }\end{array}$ \\
\hline Driving techniques & 97 & $70.3 \%$ & 1 \\
\hline Distracted & 31 & $22.5 \%$ & 2.5 \\
\hline Aggressive driving & 31 & $22.5 \%$ & 2.5 \\
\hline Purposeful violation of traffic laws & 7 & $5.1 \%$ & 4 \\
\hline Drowsy & 5 & $3.6 \%$ & 6 \\
\hline Roadway alignment & 3 & $2.2 \%$ & 7.5 \\
\hline Other & 1 & $0.7 \%$ & 7.5 \\
\hline Other emotional state & 1 & $0.7 \%$ & 5 \\
\hline
\end{tabular}




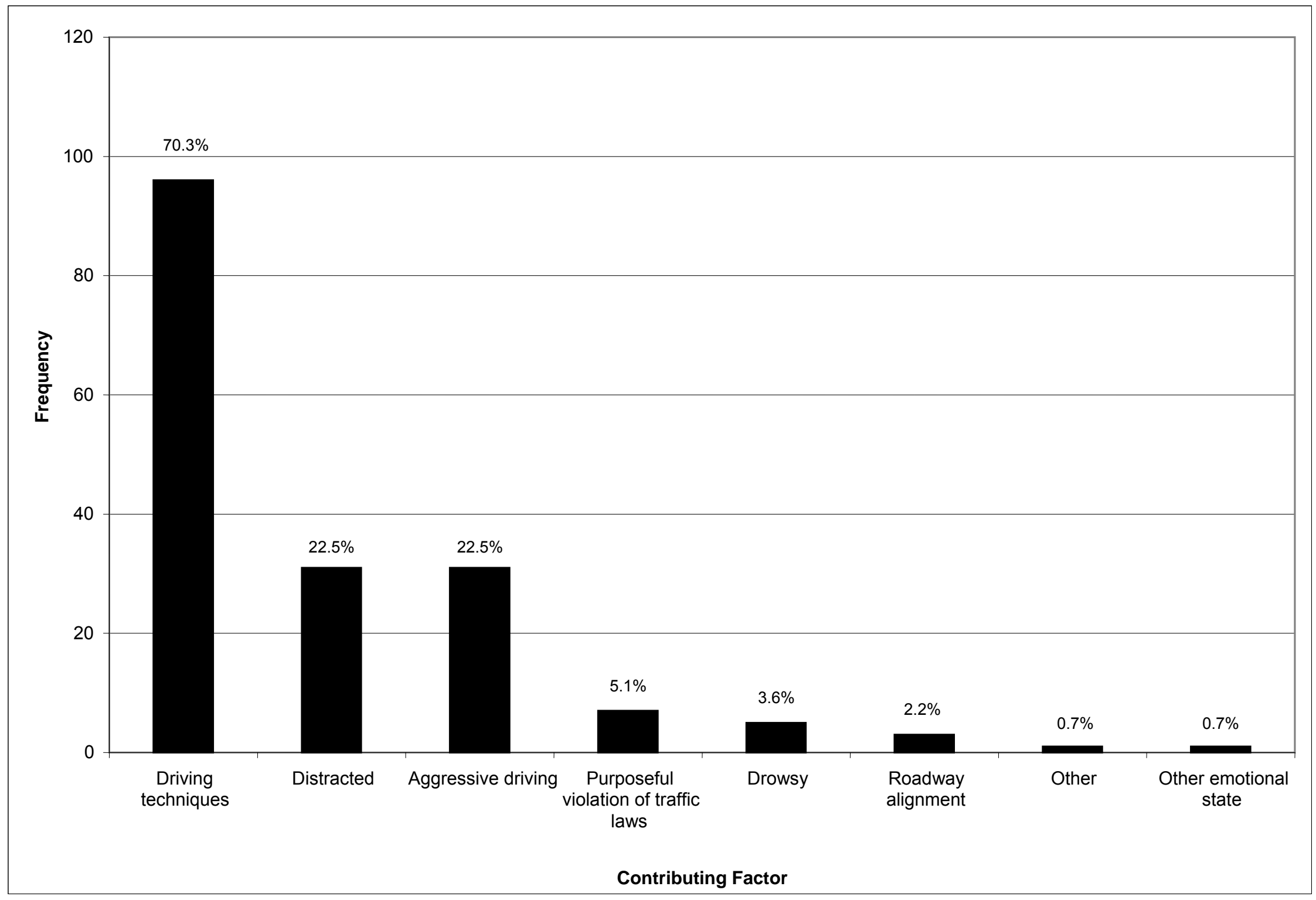

Figure 19. Frequency of Contributing Factors for LV Driver At-fault Incidents $\left(\mathbf{n}_{\mathrm{LV}}=\mathbf{1 3 8}\right)$. 
Table 20 shows the frequency, percentage, and rank ordering of each Contributing Factor for the Unknown at-fault incidents. As can be seen in Table 20, the most frequent Contributing Factor for Unknown at-fault incidents was Driving Techniques (48.3\%), followed by Distracted (20.7\%), Unknown (17.2\%), Roadway Alignment (10.3\%), and Aggressive Driving (10.3\%). Figure 20 shows a bar graph of the 29 Unknown at-fault incidents as a function of the Contributing Factor.

Table 20. Frequency, Percentage, and Rank Ordering of the Contributing Factors for the Unknown At-Fault Incidents ( $\left.\mathbf{n}_{U n}=29\right)$.

\begin{tabular}{|l|c|c|c|}
\hline \multicolumn{1}{|c|}{ Contributing Factors } & $\begin{array}{c}\text { Frequency of } \\
\text { Unknown Driver } \\
\text { At-Fault } \\
\text { Incidents } \\
\text { (nun } \mathbf{2 9})\end{array}$ & $\begin{array}{c}\text { Percentage of } \\
\text { Unknown Driver } \\
\text { At-Fault } \\
\text { Incidents } \\
\text { (nun } \mathbf{2 9})\end{array}$ & $\begin{array}{c}\text { Combined Rank } \\
\text { of Unknown } \\
\text { Driver At-Fault } \\
\text { Incidents }\end{array}$ \\
\hline Driving techniques & 14 & $48.3 \%$ & 1 \\
\hline Distracted & 6 & $20.7 \%$ & 2 \\
\hline Unknown & 5 & $17.2 \%$ & 3 \\
\hline Roadway alignment & 3 & $10.3 \%$ & 4.5 \\
\hline Aggressive driving & 3 & $10.3 \%$ & 4.5 \\
\hline Drowsy & 2 & $6.9 \%$ & 6.5 \\
\hline Roadway delineation & 2 & $6.9 \%$ & 6.5 \\
\hline Angry & 1 & $3.4 \%$ & 7.5 \\
\hline Purposeful violation of traffic laws & 1 & $3.4 \%$ & 7.5 \\
\hline
\end{tabular}


lil1..... 


\section{Contributing Factors X Incident Type Summary}

Table 21 illustrates the frequency of LV driver at-fault incidents by Incident Type as well as Contributing Factor. Table 21 provides a more descriptive and comprehensive illustration of the Contributing Factors for each Incident Type. The far left column lists the Incident Types while the Contributing Factors are listed in the first row. As indicated above, more than one Contributing Factor could be coded to a particular incident, thus, 176 occurrences of Contributing Factors were coded to $138 \mathrm{LV}$ driver at-fault incidents.

As can be seen in Table 21, the most frequent Contributing Factors for the Late Braking for Stopped/Stopping Traffic Incident Type was Driving Techniques $(n=47)$ and Distracted $(n=$ 20). The Driving Techniques and Distracted Contributing Factors were coded in $82.5 \%$ and $35.1 \%$ of the LV at-fault Late Braking for Stopped/Stopping Traffic Incident Types, respectively. The most frequent Contributing Factors for the Lane Change Without Sufficient Gap Incident Types were Aggressive Driving $(n=16)$ and Driving Techniques $(n=14)$. The Aggressive Driving and Driving Techniques Contributing Factors were coded in 53.3\% and 46.7\% of the LV at-fault Lane Change Without Sufficient Gap Incident Types, respectively. 
Table 21. Frequency of Incident Types X Contributing Factors Across LV Driver At-Fault Incidents $\left(\mathbf{n}_{\mathrm{LV}}=138\right)$.

\begin{tabular}{|c|c|c|c|c|c|c|c|c|c|c|}
\hline \multirow[b]{2}{*}{ Incident Types } & \multirow[b]{2}{*}{ 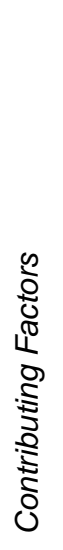 } & \multicolumn{4}{|c|}{ Driver Impairment } & \multirow{2}{*}{ 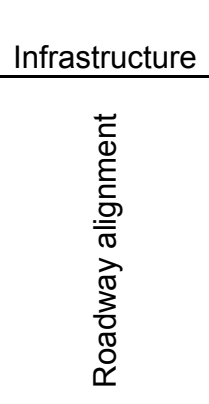 } & \multicolumn{2}{|c|}{ Willful Behavior } & \multirow{2}{*}{ 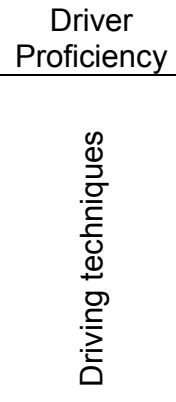 } & \\
\hline & & 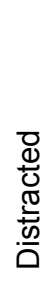 & 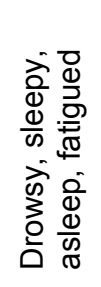 & $\frac{\grave{\Phi}}{\stackrel{ \pm}{0}}$ & 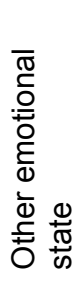 & & 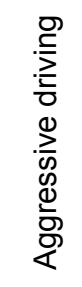 & 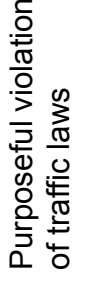 & & \\
\hline \multicolumn{2}{|c|}{ Aborted Lane Change } & 1 & 0 & 0 & 0 & 0 & 1 & 1 & 9 & 12 \\
\hline \multicolumn{2}{|c|}{ Approaches Traffic Quickly } & 1 & 0 & 0 & 0 & 0 & 1 & 0 & 5 & 7 \\
\hline \multicolumn{2}{|c|}{ Conflict With Oncoming Traffic } & 1 & 0 & 0 & 0 & 1 & 2 & 0 & 0 & 4 \\
\hline \multicolumn{2}{|c|}{ Following Too Closely } & 2 & 0 & 0 & 0 & 0 & 0 & 0 & 4 & 6 \\
\hline \multicolumn{2}{|c|}{ Improper Lane Change } & 0 & 0 & 0 & 0 & 0 & 2 & 0 & 2 & 4 \\
\hline \multicolumn{2}{|c|}{ Improper Passing } & 0 & 1 & 0 & 0 & 0 & 4 & 2 & 6 & 13 \\
\hline \multicolumn{2}{|c|}{ Improper Stopping at an Intersection } & 1 & 0 & 0 & 0 & 1 & 0 & 0 & 2 & 4 \\
\hline \multicolumn{2}{|c|}{ Lane Change Without Sufficient Gap } & 3 & 0 & 0 & 0 & 0 & 16 & 0 & 14 & 33 \\
\hline \multicolumn{2}{|c|}{ Late Braking for Stopped/Stopping Traffic } & 20 & 2 & 1 & 1 & 0 & 3 & 2 & 47 & 76 \\
\hline \multicolumn{2}{|c|}{ Lateral Deviation of Through Vehicle } & 1 & 0 & 0 & 0 & 0 & 0 & 0 & 3 & 4 \\
\hline \multicolumn{2}{|c|}{ Merge without Sufficient Gap } & 0 & 2 & 0 & 0 & 0 & 1 & 2 & 2 & 7 \\
\hline \multicolumn{2}{|c|}{ Roadway Entrance Without Clearance } & 0 & 0 & 0 & 0 & 1 & 0 & 0 & 2 & 3 \\
\hline \multicolumn{2}{|c|}{ Roadway Entrance Without Clearance } & 1 & 0 & 0 & 0 & 0 & 1 & 0 & 1 & 3 \\
\hline \multicolumn{2}{|l|}{ Total } & 31 & 5 & 1 & 1 & 3 & 31 & 7 & 97 & 176 \\
\hline
\end{tabular}


Tree Taxonomy

Figures 21-23 illustrate another way of viewing the data in Figures 18-20. Note that the numbers in the contributing factors boxes do not necessarily sum to the total in the higher at-fault category as multiple contributing factors could have been selected for each incident.

Figure 21 is a tree taxonomy of the General and Specific Contributing Factors for the 79 incidents where the $\mathrm{HV}$ driver was at-fault. An important point for the HV driver at-fault incidents is that the Contributing Factors are for the LV driver and not the HV driver. Again, this is due to the methodology used where cameras and other data collection equipment were in the LVs only. As such, the Contributing Factors in Figure 29 should be considered driver behaviors identified when the LV driver was responding to the at-fault $\mathrm{HV}$ driver.

Figure 22 provides a tree taxonomy of the 138 incidents where the LV was at-fault and provides a tree taxonomy of the 29 incidents where it was unknown if the HV or LV drivers was at-fault.

\section{HV driver at-fault Incidents}

13 Driver Impairment
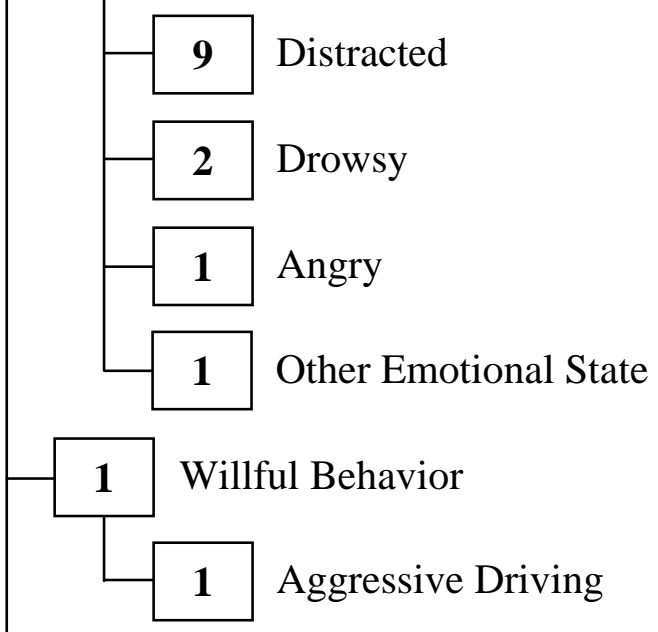

12 Driver Proficiency

12 Driving Techniques

3 Infrastructure

2 Roadway Alignment

1 Roadway Delineation

54 Unknown

Figure 21. Taxonomy of the Contributing Factors for LV Drivers in the HV At-Fault Incidents $\left(\mathbf{n}_{\mathrm{HV}}=\mathbf{7 9}\right)$. 


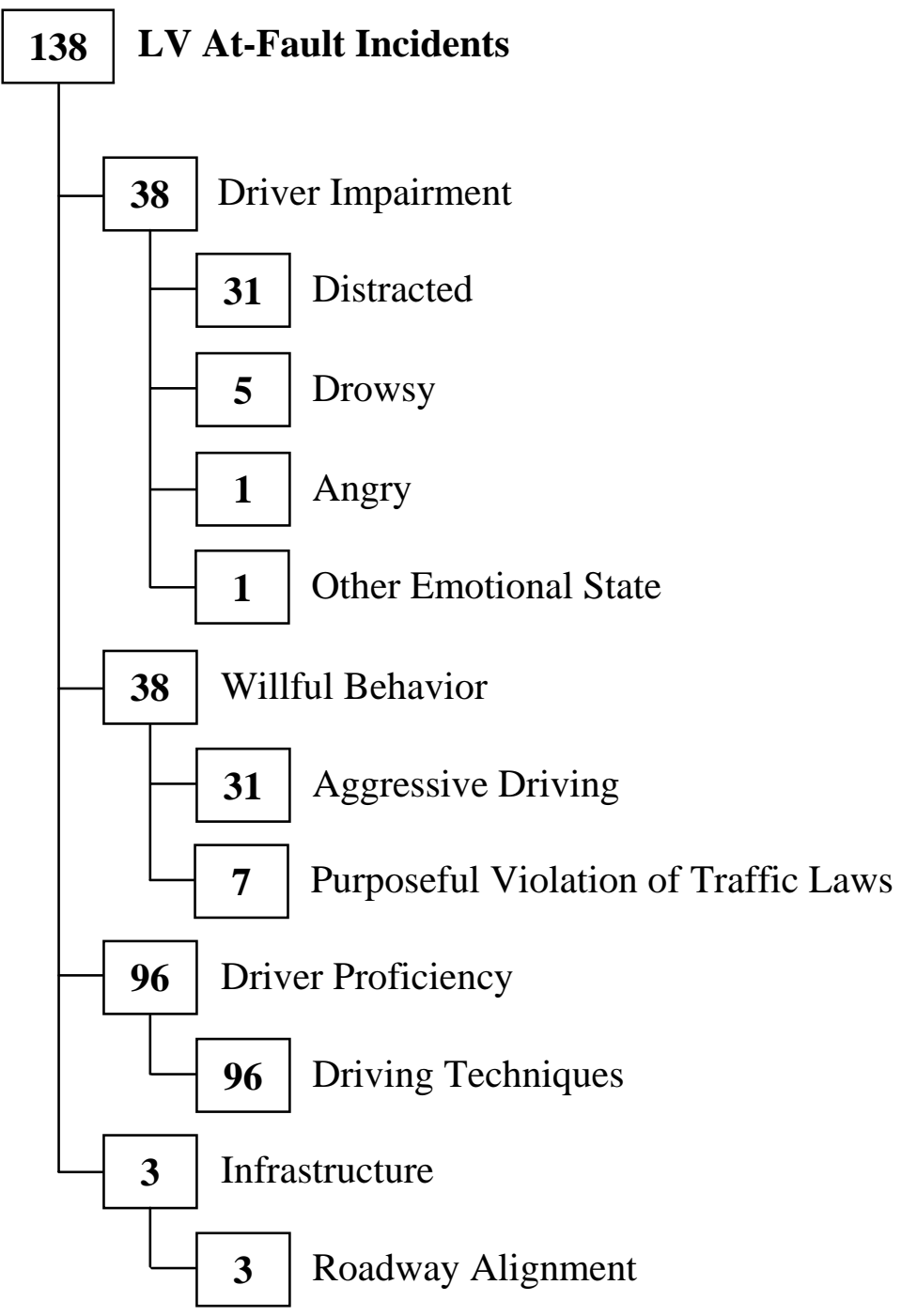

Figure 22. Taxonomy of the Contributing Factors for LV Driver At-Fault Incidents $\left(\mathrm{n}_{\mathrm{HV}}=\right.$ 138). 


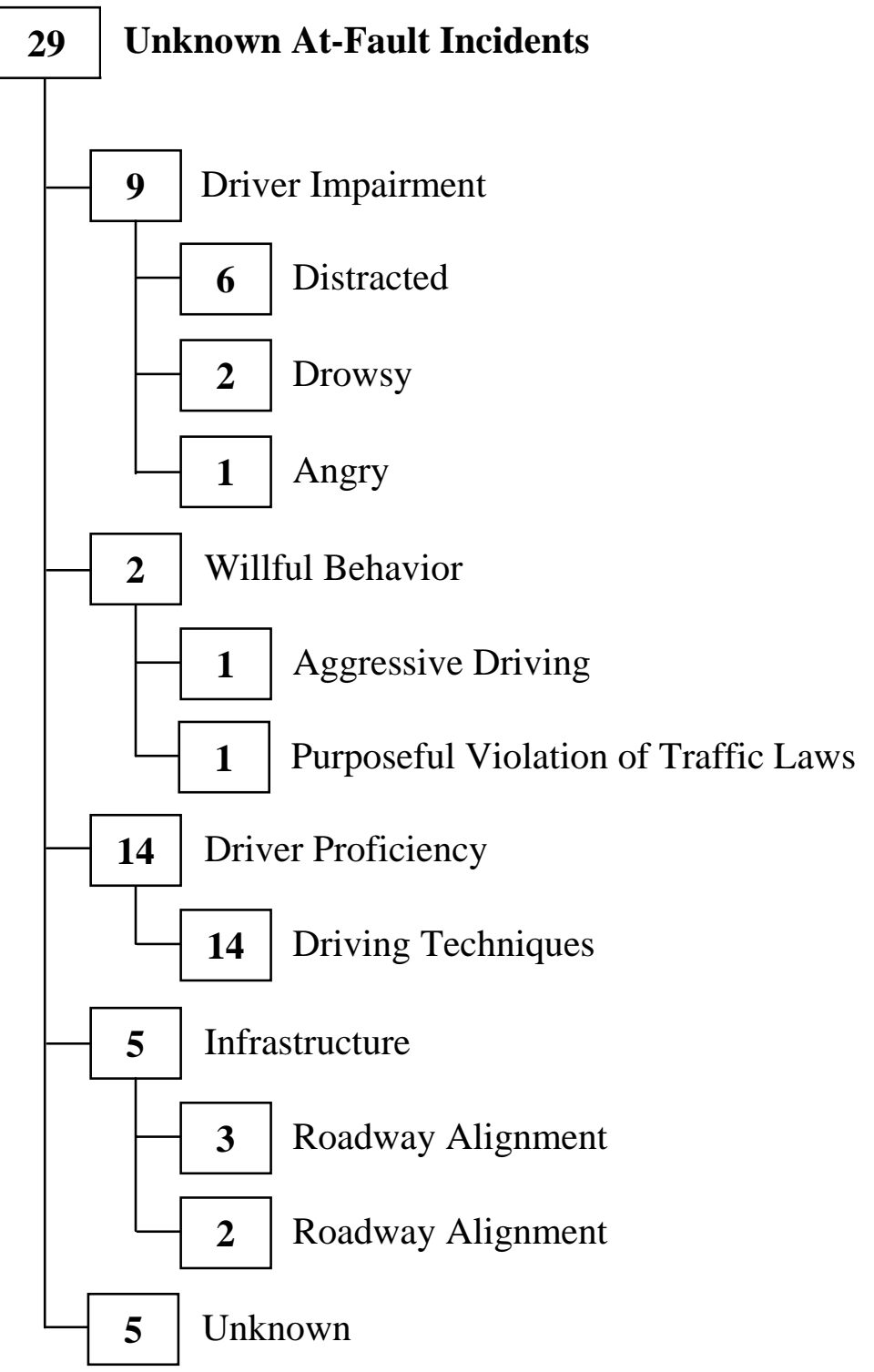

Figure 23. Taxonomy of the Contributing Factors for Unknown At-Fault Incidents $\left(\mathbf{n}_{U n}=29\right)$.

Contributing Factor Taxonomy

A taxonomy to highlight the characteristics of the incidents was also developed. The Incident Types, Contributing Factor Categories, and brief descriptions of the incidents were arranged hierarchically to create three taxonomies: (1) Figure 24 illustrates the HV driver at-fault incidents, (2) Figure 25 illustrates the LV driver at-fault incidents, and (3) Figure 26 illustrates the Unknown at-fault incidents. At the highest level of the taxonomy, the events are grouped by the driver who was at-fault for the incident (i.e., HV, LV, or Unknown). The second highest level in the taxonomy is the Incident Type, followed by the General Contributing Factor, Specific Contributing Factor, and brief description of the incident, respectively. The incident descriptions explain the basic cause and result of the incidents. The taxonomy structures are shown below in Figures 24-26. 
GENERAL

ONTRIBUTING

FACTOR

SPECIFIC

CONTRIBUTING

FACTOR

(4) Aborted
Lane Change

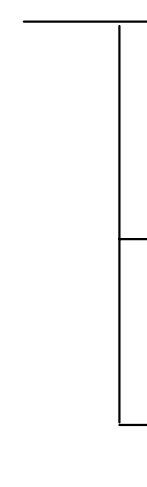

(2) Driver Impairments-

(1) Distracted

(2) Driver Impairments

(1) Drowsy

(2) Unknown

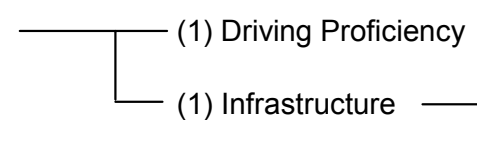

(1) Driving Techniques

\section{(1) Backing in}

Roadway

(1) Infrastructure

(1) Roadway Delineation

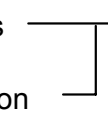

(1) Clear Path

for Emergency

Vehicle

\section{(1) Conflict with \\ Oncoming}

Traffic
(1) Unknown

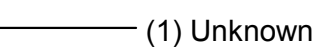

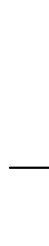

EVENT DESCRIPTION

(1) The LV is traveling to the right of an HV in traffic.

The HV driver begins to make a lane change into the LV driver's lane, causing the LV driver to brake and swerve to the right to avoid the HV.

(1) The LV is traveling to the left of an HV. The HV driver begins to change lanes into the LV driver's lane, causing the LV driver to brake hard and change lanes to the left to avoid the HV.

(2) The LV is traveling to the right of an HV. As the LV approaches the side of the HV, the HV driver begins to change lanes into the LV driver's lane. The LV driver brakes and swerves around the edge of the HV.

(1) The LV driver makes a left turn at an intersection and approaches a cone in the middle of the road. The LV driver stops and backs up in the middle of the road. There is an HV that has approached from behind and it also has to back up.

(1) An LV is traveling on a straight road. An HV makes a left turn from a side road with its lights on in front of the LV, causing the driver to brake to let the HV go.

(1) The LV driver has just made a left turn onto a side road. The LV approaches an oncoming HV that is in the middle of the road. The LV driver stops until the HV moves over and passes the LV.

Figure 24. Taxonomy Structure Used to Characterize the HV Driver At-Fault Incidents $\left(\mathbf{n}_{\mathrm{HV}}=\mathbf{7 9}\right)$. 


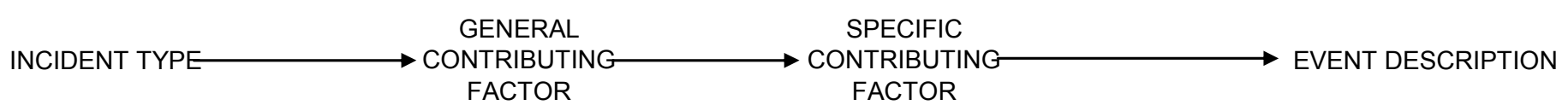

(1) Following Too

Closely

(1) Improper Lane

Change

(1) Imprope

U-turn

(1) Unknown

(1) Driving Techniques

(1) The LV is traveling behind an HV. The LV driver has to brake hard when the HV slows because the LV is

following too close behind the HV.

(1) The LV is traveling ahead of an HV as they are on an entrance ramp. As the vehicles merge into traffic, the $\mathrm{HV}$ driver changes lanes to the left and speeds up next to the LV blocking the HV from making a lane change which is the intention.

(1) The LV is traveling on a straight road; the LV approaches an HV trying to make a U-turn and is blocking the driver's path. The LV has to slow until the HV straightens out and continues.

(1) Improperly Covered Debris
(1) The LV is traveling to the left and behind an HV with an uncovered load. Debris flies off of the back of the HV startling the LV driver and causing the LV to swerve in their lane slightly before the LV accelerates to pass the HV.

Figure 24. (Continued.) 
INCIDENT TYPE
GENERAL

FACTOR
SPECIFIC

$\rightarrow$ CONTRIBUTING

FACTOR
(21) Lane Change Without Sufficient Gap
(3) Driver Impairments

(3) Distracted

(1)

(2) Willful Behavior

1) Driver Proficiency (1) Driving Techniques (2) Aggressive Driving

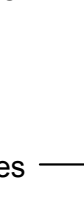

(15) Unknown
EVENT DESCRIPTION

(3) An HV to the right and slightly ahead of the LV changes lanes to the left with very little room in front of the LV, startling and causing them to swerve to the left.

(2) The LV is traveling behind and to the left of an HV. The HV changes lanes to the left in front of the LV. causing them to brake suddenly.

(1) The LV is traveling behind and to the right of an HV. The HV moves to the right in front of the LV without using a blinker, causing the LV to brake hard to avoid the truck.

(15) The LV has just moved into a right turn lane. There is an HV in the left lane who changes lanes directly in front of the LV, causing them to brake suddenly.

\section{(1) Late Braking}

for Stopped/Stopping

Traffic
(1) Driver Impairments

(1) Driver Proficiency
(1) Distracted

(1) Driving Techniques

(1) The LV is changing lanes to the right across several lanes of traffic. As they change lanes, an HV ahead of them also changes lanes from the same direction into the same lane. The subject driver has to brake as they approach the truck.

Figure 24. (Continued.) 
SPECIFIC

CONTRIBUTING

FACTOR
GENERAL

INCIDENT TYPE

Deviation

of Through

Vehicle

FACTOR
(1) Driver Techniques

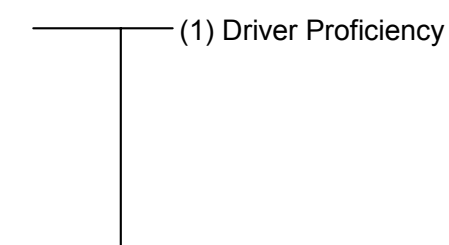

(2) Infrastructure

(2) Roadway Alignment

(14) Unknown

\section{(11) Left Turn} Without Clearance

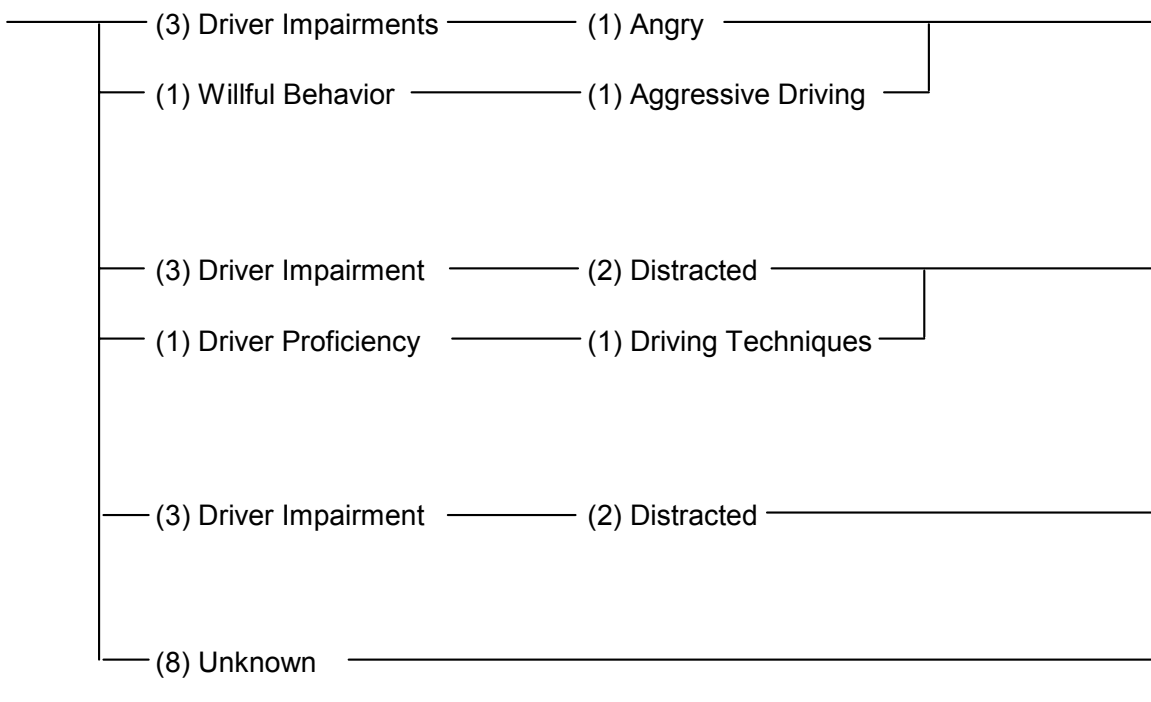

Figure 24. (Continued.)
(1) The LV is traveling to the left of a bus. The bus crosses into the LV lane. The LV swerves to the left to avoid the bus as they enter an intersection then has to swerve back to the right in front of the bus to avoid oncoming traffic.

2) The LV is in a left most lane next to concrete pillars with an HV in the lane to the right. The HV crosses over their left lane line slightly, causing the subject vehicle to brake and wait for the truck to move.

(14) The LV is traveling on an undivided single lane road. An HV, approaching from the opposite direction, crosses the center line into the LV lane.

(1) The LV is stopped at a traffic signal. The last 2 vehicles (an automobile and a bus) in oncoming traffic in the left turn lane run the red light and are in the intersection as the LV light turns green. The LV driver starts to accelerate slowly and honks the horn.

1) The LV has the right of way going straight through a traffic signal. An HV driver makes a left turn from the opposite direction without enough clearance, causing the LV driver to brake hard before the HV clears the intersection.

(1) An HV driver pulls out from the left and merges to the far right lane in front of the LV. The LV driver brakes hard to avoid hitting the HV.

(8) The LV has the right of way going straight when an oncoming HV driver makes a left turn in front of the LV causing the LV driver to brake. 


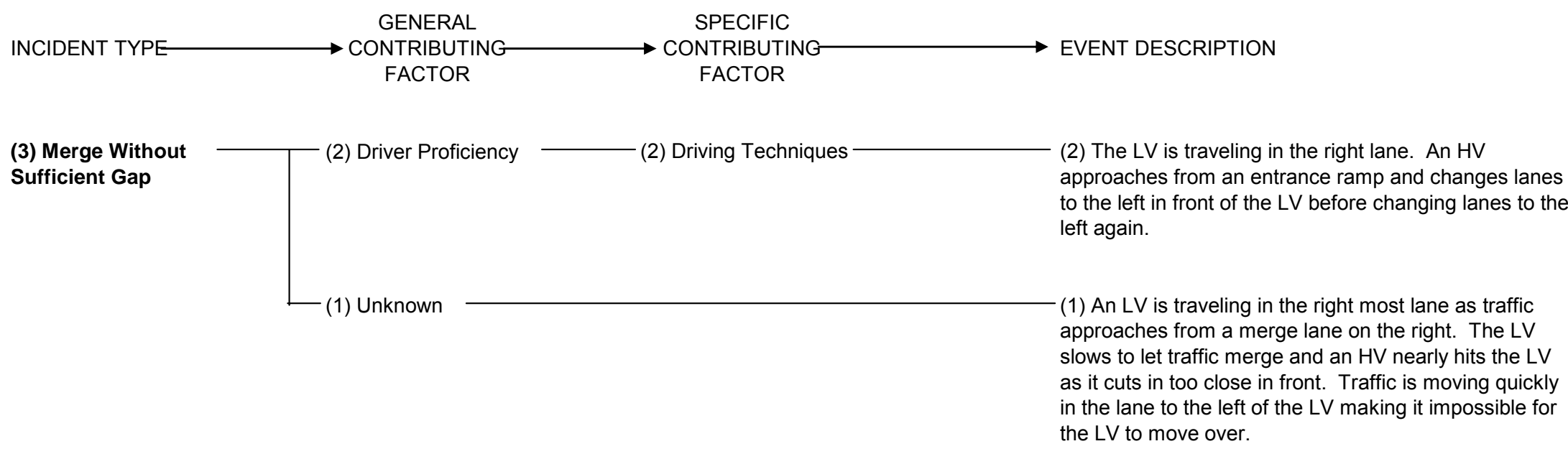

(4) Obstruction in Roadway

$\left[\begin{array}{l}\text { (1) Driver Proficiency } \\ \text { (3) Unknown }\end{array}\right.$

(3) Unknown

(1) Driving Techniques

(1) The LV approaches an HV that is parked in its traffic lane. The LV has to swerve to the left into oncoming traffic to avoid the HV, coming close to another vehicle.

3) The LV is traveling behind an HV that moves partially into a left turn lane. The LV driver has to brake and go around the HV because the HV is sticking out in the LV's

\section{(2) Roadway \\ Entrance Without}

Clearance

(1) Slow Speed lane.

(2) The LV is traveling on a straight wet road. An HV makes a right turn from a side road directly in front of the LV.

(1) The LV is traveling in the left lane on a highway. The LV approaches an HV ahead in the left lane who is traveling much slower than other traffic. The LV driver brakes hard and changes lanes to the right.

Figure 24. (Continued.) 
GENERAL

INCIDENT TYPE

$\rightarrow$ CONTRIBUTING

FACTOR

(1) Driver Proficiency

(1) Sudden Braking

in Roadway

(3) Through Traffic

Does Not Allow

Merge

$\left[\begin{array}{r}2 \\ \end{array}\right.$

(1) Driver Proficiency

(1) Driving Techniques

SPECIFIC

CONTRIBUTING

FACTOR
EVENT DESCRIPTION

(1) An LV is behind an HV whose driver brakes suddenly for a red light. The LV comes close to the HV and causes the LV driver to brake hard.

(1) The LV is trying to merge onto the highway. There is an $\mathrm{HV}$ in the lane to the left of them that will not move to let the LV merge. The LV has to move over to the shoulder to let the HV pass before the LV can merge into traffic.

(2) The LV is trying to merge onto the highway, but an $\mathrm{HV}$ in the lane to the left will not allow the LV to merge.

(1) Turn Without Sufficient Warning

(1) Turn From Incorrect Lane

(1) Driver Impairment

(1) Other Emotional State

\section{(2) Wide Turn Into} Adjacent Lane

$\left[\begin{array}{l}\hline \text { (1) Driver Impairment } \longrightarrow \text { (1) Drowsy }- \\ \text { (1) Unknown }\end{array}\right.$

Figure 24. (Continued.)
(1) The LV changes lanes to the left at the same time that the lead HV changes lanes to the left. The HV then stops and makes a right turn from the left lane, cutting off vehicles in the right lane and causing the LV driver to brake.

(1) The LV is traveling behind a HV who slows and pulls off the side of the road. The HV doesn't completely pull off the road, causing the LV driver to go slightly over the double yellow line while there is oncoming traffic.

(1) The LV is traveling on a single lane undivided road. An HV makes a wide right turn from a side road onto the LV's road (oncoming traffic) and crosses over the double yellow line. The LV stops until the HV completes the turn and returns to the HV's lane.

(1) An HV makes a left turn from a side road and enters the LV's lane slightly while turning into their lane. The LV brakes until the HV finishes the turn. 
GENERAL SPECIFIC

INCIDENT TYPE $\longrightarrow$ CONTRIBUTING $\longrightarrow$ CONTRIBUTING

FACTOR FACTOR

\begin{abstract}
(11) Aborted
(2) Driver Impairments

(1) Distracted

Lane Change

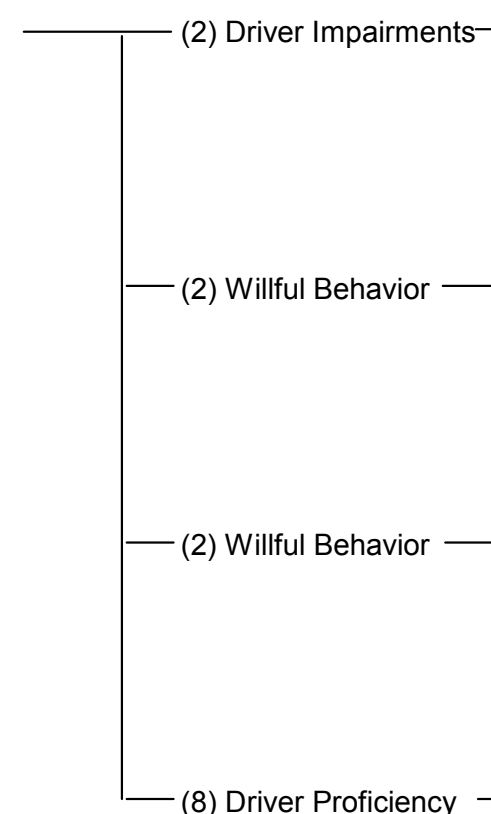

(1) Aggressive Driving

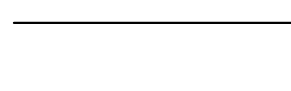

The LV driver is changing lanes to the right across several lanes of traffic. As they begin to change lanes again, an HV changes lanes from the left into the same lane. The LV driver aborts their lane change and moves back into the original lane.

LV approaches a slow moving $H V$ and starts to As the LV driver turns their head to check the blind spot, the HV also starts to change lanes to the right. The LV driver notices this when they turn back and move back into the lane.

8) Driver Proficiency

(8) Driving Techniques

(8) The LV driver is attempting to change lanes to the right. As they begin the lane change, they notice an HV in the right lane and have to turn the wheel quickly to stay in their lane.
\end{abstract}

EVENT DESCRIPTION

Figure 25. Taxonomy Structure Used to Characterize the LV Driver At-Fault Incidents $\left(\mathrm{n}_{\mathrm{LV}}=\mathbf{1 3 8}\right)$. 


GENERAL
CONTRIBUTING
FACTOR $\underset{\substack{\text { CONTRIBUTING } \\ \text { FACTOR }}}{\text { SPECIFIC }} \longrightarrow$ EVENT DESCRIPTION

(6) Approaches

Traffic Quickly

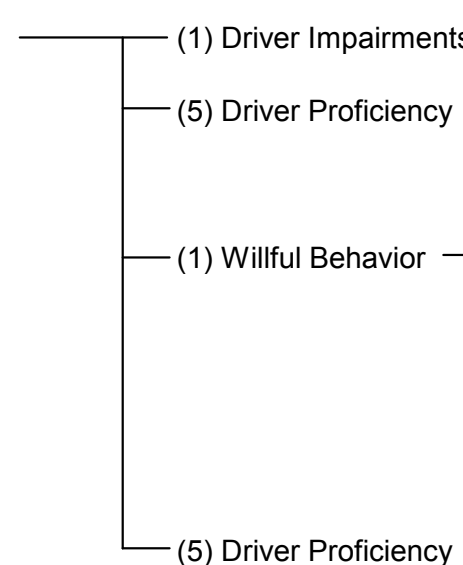

(1) Distracted

(5) Driving Techniques

(1) Aggressive Driving

5) Driving Techniques
(1) The LV approaches an HV that is stopped while delivering mail. The LV driver is distracted while trying to light a cigarette and approaches the HV quickly. The LV driver has to brake moderately hard to avoid the HV.

(1) The LV is changing lanes to the right across two lanes of traffic to get into an exit lane. As the LV driver enters the exit lane, they change lanes behind an HV driver who is braking for the exit. The LV was accelerating during the lane changes and now the LV driver has to brake hard behind the truck.

(4) The LV is traveling behind an HV driver who is at the end of a line of traffic. The LV driver accelerates up to the traffic then has to brake suddenly as they notice the traffic is nearly stopped.

\section{(3) Conflict with}

Oncoming

Traffic

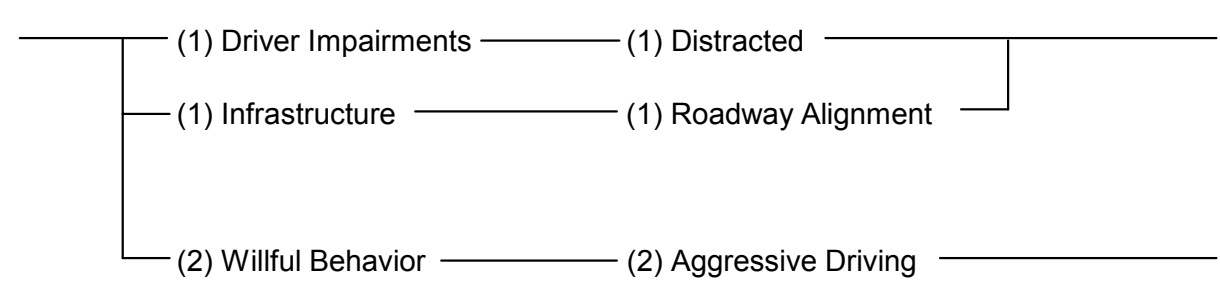

(2) Willful Behavior

(2) Aggressive Driving

1) The LV driver has just made a left turn onto a side road. They approach an oncoming HV that is driving in the middle of the road. The LV driver stops until the HV moves over and passes them.

(2) The LV is traveling on a single lane undivided road behind a slow HV. During a passing zone, they move to the left to pass the HV, but have to brake and move back behind the $\mathrm{HV}$ due to an oncoming car.

\section{Figure 25. (Continued.)}




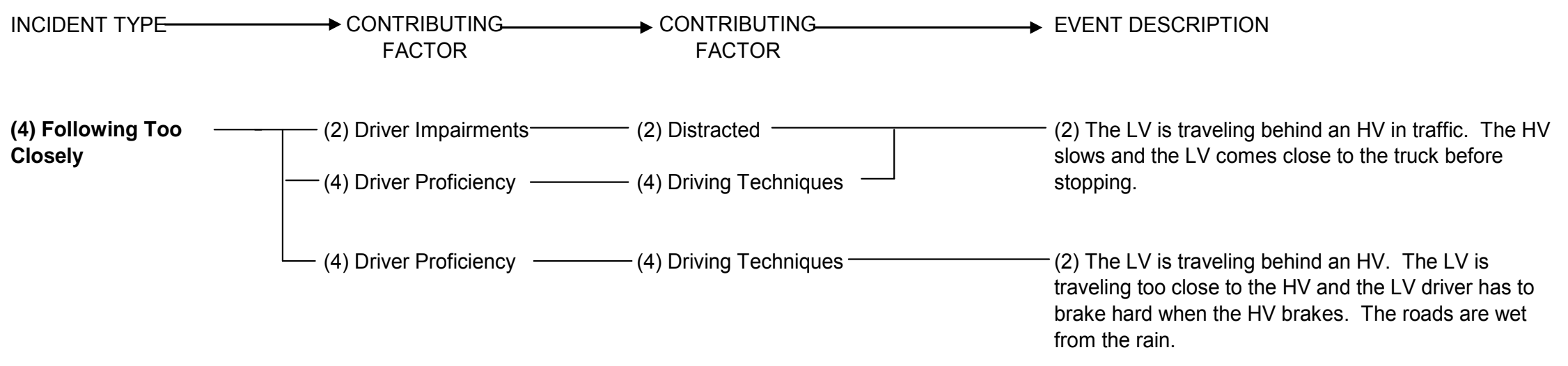

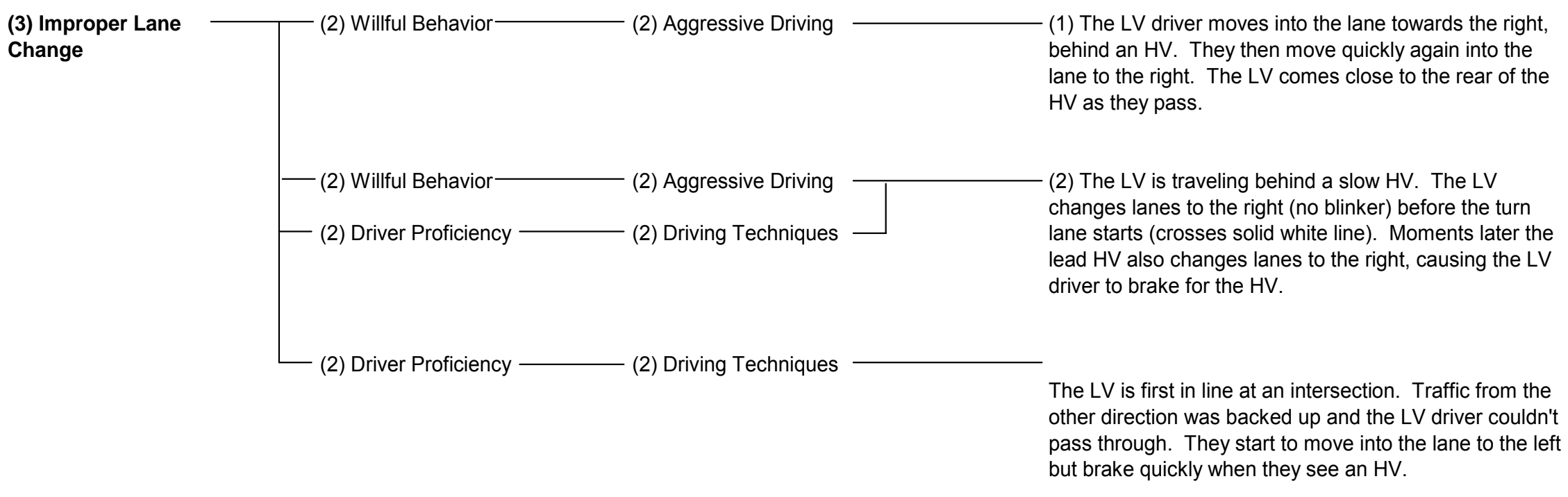

Figure 25. (Continued.) 


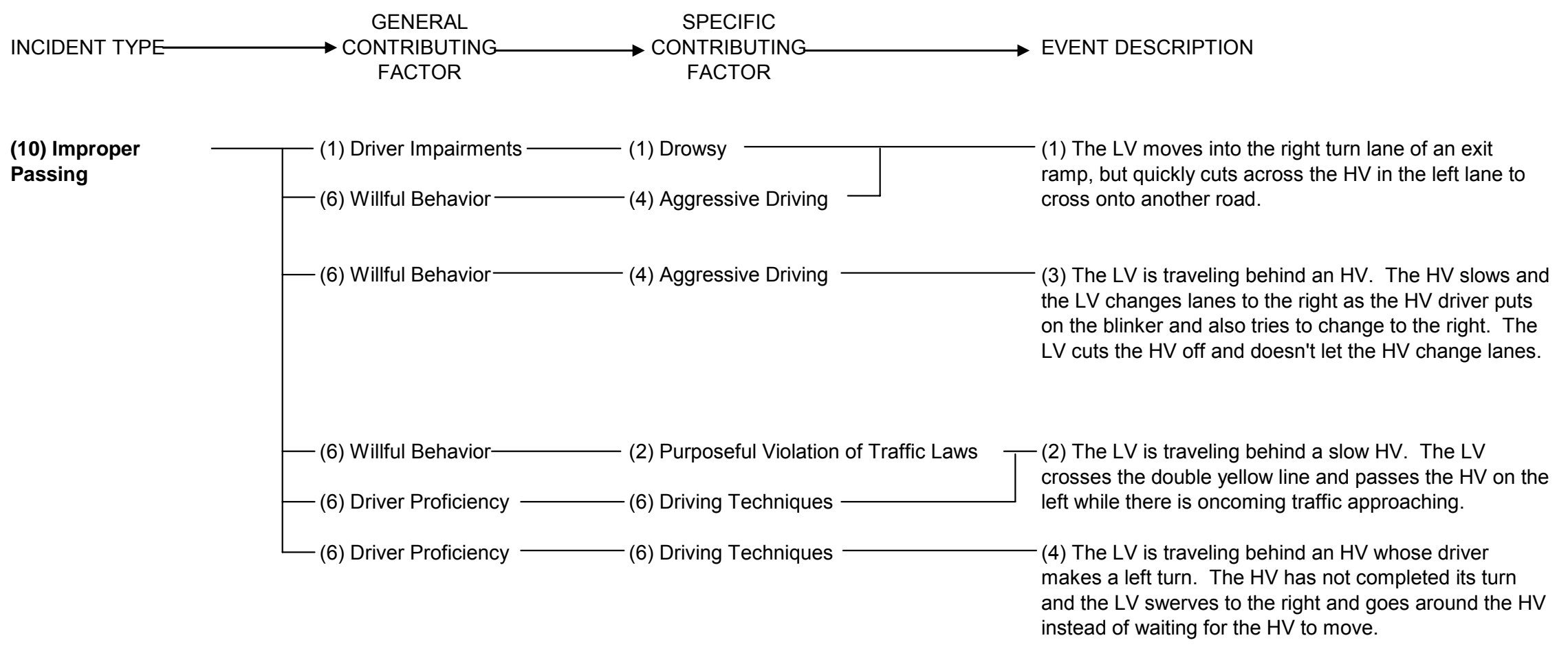

\section{(2) Improper Stopping at an} Intersection

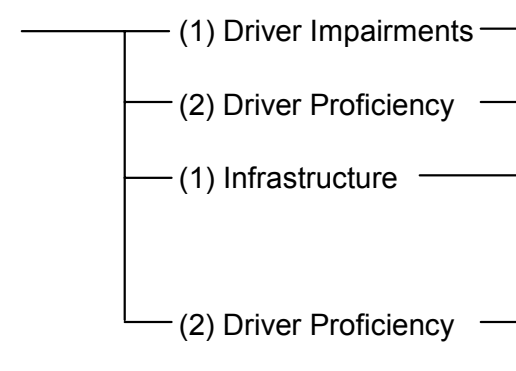

(1) Distracted

(2) Driver Techniques

(1) Roadway Alignment

(2) Driver Techniques
(1) The LV approaches a stopped HV at a traffic signal. The HV is the first at the light so the HV continues to the white line and stops. There is a perpendicular lane in the middle of the LV's lane and they are suppose to stop at a second white line to let vehicles pass.

(1) The LV stops quickly at a stop sign. The following HV has to brake hard to stop behind the LV.

Figure 25. (Continued.) 
GENERAL

INCIDENT TYPE

FACTOR

SPECIFIC

CONTRIBUTING

FACTOR

(30) Lane Change Without Sufficient Gap

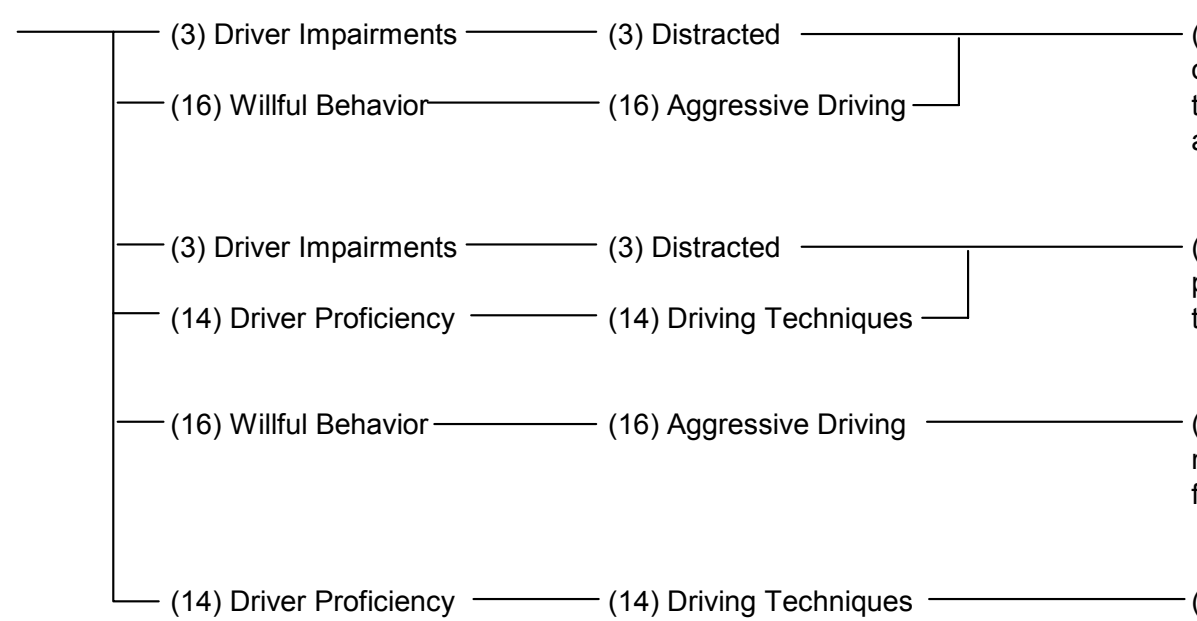

(14) Driver Proficiency

(14) Driving Techniques

(12) The LV is traveling in the right lane behind slow

(1) The LV driver is traveling in slow traffic while talking on a cell phone. The LV makes a quick lane change to the left, coming close to the lead HV as the LV changes and cuts in close in front of another HV.

(2) The LV driver is distracted while talking on the phone. They change lanes to the right close between two HVs.

(15) The LV is traveling behind a slow vehicle. The LV makes a quick lane change to the right, cutting off the following $\mathrm{HV}$ in the new lane. traffic and changes lanes to the left in front of an HV. for Stopped/Stopping

Traffic
(57) Late Braking
(22) Distracted

(24) Driver Impairments

26) Driver Impairments

(1) Other

五



C.

(5) The LV is traveling behind an HV in a construction zone. The HV driver brakes, causing the LV driver to brake. The LV driver is distracted by adjusting the radio prior to the event.

(1) The LV approaches an HV waiting to make a right turn. The LV driver comes close to the HV and has to brake suddenly as they approach.

(1) The LV is traveling behind an HV. The LV driver has to brake moderately hard and comes close to the HV as traffic slows.

(17) The LV is traveling behind an HV driver who is braking for traffic. The LV driver seems to be distracted and has to brake hard behind the HV.

\section{Figure 25. (Continued.)}


GENERAL

INCIDENT TYPE-

57) Late Braking

for Stopped/Stopping

Traffic (con't)
SPECIFIC

CONTRIBUTING

FACTOR
EVENT DESCRIPTION

(2) A LV is traveling behind an HV who is decelerating to make a left turn. The LV driver has to brake hard and comes close to the HV as the HV is turning. The LV driver looks slightly drowsy during this event.

(2) The LV is traveling behind a slow HV so the LV starts to make a lane change to the right but there is a stopped HV so they move back into their lane. When they move back the lead HV stops and the LV driver has to brake hard behind the HV.

\begin{tabular}{l} 
- (5) Willful Behavior \\
\hline (47) Driver Proficiency \\
\hline (47) Driver Proficiency \\
(5)
\end{tabular}

(3) Aggressive Driving

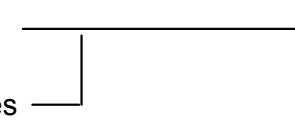

(1) The LV is attempting to change into the right lane Halfway through the lane change, an HVdriver in the right lane brakes causing the LV driver to brake hard before completing the lane change.

26) The LV is traveling behind an HV trailing a vehicle. The HV driver slows and makes a right turn onto a side road. The LV driver brakes hard behind the HV.

(5) Willful Behavior

(2) Purposeful Violation of Traffic Laws

(2) The LV makes a lane change to the left into a lane where there is an HV stopped in traffic. The LV driver has plenty of room to complete the lane change, but has to brake hard as they approach the stopped HV.

\section{Figure 25. (Continued.)}


GENERAL

INCIDENT TYPE

Deviation

of Through

Vehicle

(5) Merge Without Sufficient Gap
SPECIFIC

CONTRIBUTING

FACTOR

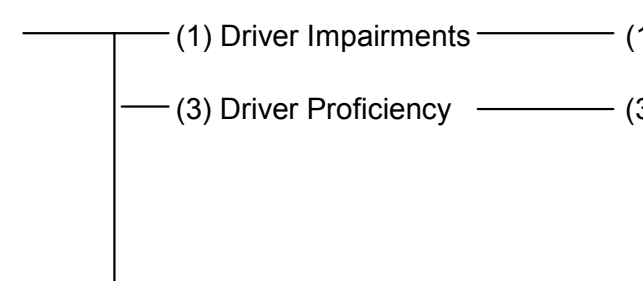

(3) Driver Proficiency

(3) Driver Techniques

(3) Driver Techniques

(2) Drowsy

(2) Driver Impairments

$\bar{z}$

(3) Driver Proficiency
(2) Driver Impairments

(3) Driver Proficiency

(3) Driver Techniques

(3) Willful Behavior

(2) Purposeful Violation of Traffic Laws

(3) Driver Techniques

Figure 25. (Continued.)
EVENT DESCRIPTION

(1) An LV driver is very distracted with something in their lap. The LV is driving on a single lane undivided road and crosses over the double yellow line on a curve. An $\mathrm{HV}$ comes in the opposite direction and the LV driver swerves quickly to the right to avoid the HV.

(2) The LV is traveling in the left lane on a two lane undivided highway. The LV is slightly over the double yellow line when an oncoming HV passes. The LV brakes and moves to the right to return to the LV's lane.

1) The LV is in the right most lane which is ending. The $V$ does not merge to the left in time and gets cut off by an HV. The LV driver has to brake and wait for the HV to pass before moving over.

(1) The LV is in the far right lane which is partially blocked by barrels. There is an HV in the lane to the left of the LV which the LV is trying to get in front of. The barrels block the LV's lane before they can merge over and the LV starts to hit the barrels.

(2) The LV is in slow traffic. The vehicle in front of them stops to merge to the left into traffic, and the LV goes around the stopped car on the right and tries to merge into traffic on the left behind an HV.

(1) The LV is in an entrance lane and begins to merge before the lead vehicle merges. There is an HV approaching from behind and the LV driver brakes and swerves a little before the HV driver lets them in. 
GENERAL

INCIDENT TYPE

(2) Roadway

Entrance Without

Clearance

(2) School Bus

Passing Violation
SPECIFIC

CONTRIBUTIN

FACTOR
EVENT DESCRIPTION

(1) The LV makes a right turn onto a road in front of an $H V$. The LV has to swerve as the HV approaches from behind.

\begin{abstract}
(1) An LV is stopped at a stop sign preparing to merge onto a busy road. The LV driver has their head turned to the left to check traffic as a truck approaches from the right. The subject begins to move, then notices the truck and has to brake suddenly.

(2) Driver Proficiency

(2) Driving Techniques

(1) Roadway Alignment

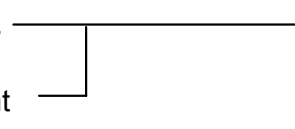

(1) Infrastructure
\end{abstract}

(1) Distracted

(1) Driver Techniques

$\longrightarrow$

(1) LV does not stop for the stopped HV (school bus) on the opposite direction which displays a stop sign. The

LV driver later received a ticket for this matter.

(1) Willful Behavior

(1) Aggressive Driving

(1) The LV approaches a stopped HV (school bus) with the stop arm extended. The LV passes the school bus on the left without stopping

Figure 25. (Continued.) 


GENERAL
INCIDENT TYPE
FACTOR $\underset{\substack{\text { CONTRIBUTING } \\ \text { FACTOR }}}{\text { SPECIFIC }} \longrightarrow$ EVENT DESCRIPTION
(1) Clear Path
(1) Willful Behavior
(1) Aggressive Driving
(1) The LV is in the far left lane when an HV
for Emergency
Vehicle
(1) The LV has to move across two lanes of traffic onto the right shoulder. (4) Conflict with
Oncoming Traffic

(2) Improper

Passing

(3) Lane Change

Without Sufficient

Gap

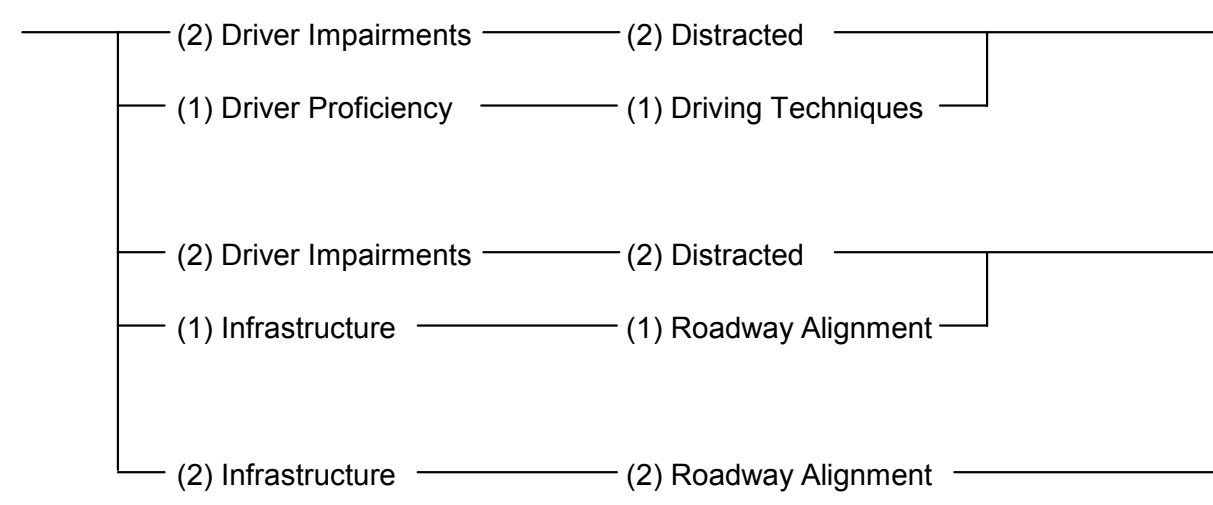

(2) Infrastructure

(2) Roadway Alignment

(1) The LV is traveling on an undivided road. The driver approaches an oncoming $\mathrm{HV}$ and swerves to the right to avoid the HV, then has to swerve back to the left to avoid a parked car on the side of the road.

1) The LV driver is on a back, curvy road when they approach an HV coming from the opposite direction as the LV comes around a curve. The LV driver has to brake and move over to the far right of the road to pass the HV.

(2) The LV is traveling on a narrow back road and approaches an HV coming from the opposite direction. The LV driver has to slow and move off of the side of the road to let the HV pass.

2) The LV approaches an HV who is sticking out from the left turn lane. The LV driver has to swerve around the HV slightly as they pass, crossing into the adjacent lane where there is another vehicle.

(3) The LV is traveling in the lane to the left of a stopped HV. The LV driver brakes hard as the HV starts to move. Then, the HV changes lanes to the left directly in front of the LV.

Figure 26. Taxonomy Structure Used to Characterize the Unknown At-Fault Incidents $\left(\mathbf{n}_{\mathrm{Un}}=29\right)$. 


GENERAL
INCIDENT TYPE
FACTOR $\underset{\substack{\text { CONTRIBUTING } \\ \text { FACTOR }}}{\text { SPECIFIC }} \longrightarrow$ EVENT DESCRIPTION

(8) Late Braking for Stopped/Stopping

Traffic
(2) Driver Impairments

(2) Distracted

E

( D

(2)

Driver Impairments

(2) Distracted

(5) Driver Proficiency

5) Driver Proficiency
(5) Driving Techniques

(5) Driving Techniques

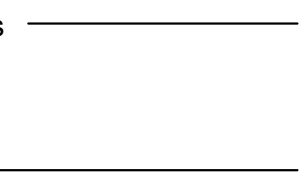

(1) The LV driver is attempting to change lanes to the right. As the LV driver turns to check the blind spot, the $\mathrm{HV}$ ahead slows, causing the LV driver to brake suddenly when they look forward again.

(1) The LV driver is traveling in traffic while operating a PDA. The LV driver brakes behind the lead vehicle, and the following HV has to brake hard behind the LV.

4) An LV is traveling behind an HV. An HV driver brakes in an exit lane so the LV driver brakes hard.

(2) The LV is traveling behind an HV in busy traffic. The HV slows and the LV driver has to brake hard and comes close to the HV as the LV slows.

(2) Left Turn
Without Clearance
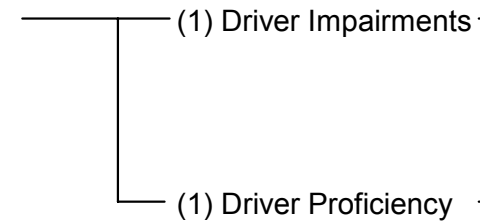

(1) Distracted

(1) Distracted

(1) Merge Without

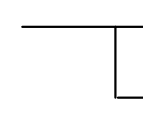

(1) Driver Impair

(1) Roadway Delieation

Figure 26. (Continued.)
(1) The LV approaches an HV which is stopped perpendicular to them in the middle of an intersection. The LV driver has to stop and wait for the HV to move.

(1) The LV is traveling straight and appears to have the right of way through an intersection. An HV makes a left turn from the opposite direction causing the subject to stop completely until the HV passes through the intersection.

(1) The LV driver is trying to pass an HV on the lef where the road merges into one lane (exit ramp). The LV driver runs out of room and brakes to let the HV pass before the LV driver merges into the single lane. 
GENERAL

ONTRIBUTIN

(1) Infrastructure

in Roadway

SPECIFIC

ONTRIBUTING

FACTOR

(1) Roadway Delineation

EVENT DESCRIPTION

(1) The LV is traveling in the left lane of a two lane undivided road. There is some construction equipment in the road blocking the lane and causing the LV driver to swerve into the right traffic lane.

\section{(2) Roadway}

Entrance Without

Clearance

$-(1)$

(1) Driver Impairments

(1) Distracted

(2) Driver Proficiency

(2) Driving Techniques

(1) The LV appears to have the right of way traveling straight. An HV enters from a side road in the far right lane causing the LV driver to brake hard because the LV driver thinks the HV is too close to them.

(1) Willful Behavior

(1) Purposeful Violatiton of Traffic Laws

(1) The LV is traveling on a single lane, undivided road. The LV approaches an HV who is moving onto the road (from the side of the road). The LV brakes hard behind the HV, then the HV brakes and the LV crosses the double yellow line to go around the LV.

(2) Through Traffic Does Not Allow Lane Change

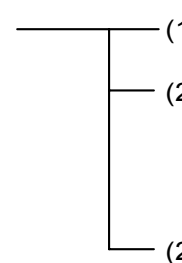

(2) Driving Techniques

Driver Impairments

2) Driver Proficiency

(1) Drowsy

Techniques

(2) Driving Techniques

(1) The LV is trying to merge into traffic on the highway. There is an HV in the lane to the left of the LV that does not move over to allow the LV to change lanes. The LV driver has to brake and swerve around a little in the lane, coming close to the guardrail.

1) The LV is traveling to the left and behind an HV. The HV's left blinker has been on for some time and the LV has plenty of room to move over but doesn't. The HV finally moves over to the left lane and the LV changes lanes to the left.

\section{(3) Unable to \\ Determine}

(2) Driver Proficiency

(2) Driver Proficiency
(1) Angry

(2) Driving Techniques

(2) Driving Techniques

Figure 26. (Continued.)
(1) The LV approaches an HV stopped at a traffic signal. The LV driver is confused as to what the HV is doing and tries to swerve to the left. The HV starts moving and both vehicles make a left turn.

2) The LV is in a double left turn lane with an HV to their left. The LV driver steers to the left to avoid the HV, but comes close to the median, and has to steer sharply to the right to avoid hitting the median. 


\section{Contributing Factors Summary}

Overall, the most frequent Contributing Factors were Driving Techniques (49.5\%), Unknown (24\%), Distracted (18.7\%), and Aggressive Driving (15\%). The most prevalent Contributing Factors for HV driver at-fault incidents were Unknown (68.4\%), Driving Techniques (15.2\%), and Distracted (11.4\%). The most prevalent Contributing Factors for LV driver at-fault incidents were Driving Techniques (70.3\%), Distracted (22.5\%), and Aggressive Driving (22.5\%). Please note that all Contributing Factors were coded with respect to LV driver at-fault incidents.

These findings are similar to what Hankey et al. (1999) found when they conducted a database analysis using the state of Pennsylvania's crash database. The Hankey et al. (1999) study found that 77\% of crashes occurring from 1995 to 1996 had "human error" cited as a primary factor in the crashes. By summing the frequency of incidents that had at least one Contributing Factor associated with human error in Figures 18 and 19 (e.g., Driving Techniques, Aggressive Driving, and Purposeful Violation of Traffic Laws) for both HV and LV driver at-fault incidents yields a total of 145 incidents out of 217 . Thus, $66.8 \%$ of all the LV-HV interactions were determined to have at least one human error as a Contributing Factor. Yet, when only the LV driver at-fault incidents are considered, 94.2\% (130 incidents out of 138) of the incidents had at least one human error as a Contributing Factor. It should be noted that in the Hankey et al. (1999) study, human error was the primary factor (i.e., excluding all other factors) in the crash, while in the present study it was a contributing factor. Another difference is that the Hankey et al. (1999) study was a crash database analysis that included PARs, which may be biased as they rely, in part, on verbal reports from drivers. The current study had cameras inside the vehicle recording the behaviors of the driver as the incident occurred; this methodology eliminates driver verbal report bias.

Hankey et al. (1999) also conducted a database analysis using the 1996 FARS database. In this analysis, "Aggressive Driving" was found to be a primary factor in $31.1 \%$ of the fatal crashes. The current research found that Aggressive Driving contributed to $15 \%$ of all the LV-HV incidents. However, the Hankey et al. (1999) study assessed all crash types. While there are many similarities between the current data and the Hankey et al. (1999) study, the current research assessed LV-HV interactions rather than all crash types (e.g., LV-LV interactions were not considered).

Stuster (1999) assessed driver-related factors in LV-HV fatal crashes. He found that $67.3 \%$ of passenger vehicles were cited with a driver-related factor in fatal LV-HV crashes (these were similar to the Willful Behavior and Driver Proficiency categories in Contributing Factors). ${ }^{4}$ Stuster's (1999) results are similar to the results in the current research, where $66.8 \%$ of the LVHV interactions were determined to have human error as a Contributing Factor.

Yet, Stuster's (1999) analysis found that only 4.3\% of the passenger vehicles were cited with the driver-related factor "Erratic/Reckless Driving" in fatal LV-HV interaction crashes. This is substantially less than the $22.5 \%$ of LV driver at-fault incidents cited as "Aggressive Driving" in the current research. This discrepancy could highlight the differences between fatal crashes

\footnotetext{
${ }^{4}$ Passenger vehicles were only considered because the Contributing Factors in the current research were based solely on the behavior(s) of the LV driver.
} 
(such as those found in Stuster, 1999) and near crashes and incidents (such as the current research). It might also underscore the difference in methodologies (i.e., a crash database approach compared to a naturalistic or in situ data collection approach) or the fact that the current study had a disproportionate number of younger drivers who tend to drive more aggressively (Chliaoutakis et al., 2002).

\section{Driver Distraction}

A substantial number of the LV-HV incidents had Distraction listed as a Contributing Factor. Again, as indicated previously, the incidents where Driver Distraction was indicated refer to the behavior of the LV driver. The Distraction Contributing Factor was sub-divided into several discrete categories. Table 22 shows the frequency, percentage, and rank ordering for each subcategory in the Distraction Contributing Factor. As can be seen in Table 22, the most frequent sub-category for the Distraction Contributing Factor was Talking/Listening on Cell Phone (21.7\%), followed by Passenger in Adjacent Seat (13\%) and Dialing Hand-Held Phone (8.7\%). Figure 27 displays a bar graph of the 46 Distraction Contributing Factors as a function of the discrete sub-categories.

Table 22. Frequency, Percentage, and Rank Ordering of Each Sub-Category in the Distraction Contributing Factor $(n=46)$.

\begin{tabular}{|c|c|c|c|}
\hline Distraction & $\begin{array}{l}\text { Frequency of } \\
\text { Distraction } \\
\text { Incidents }\end{array}$ & $\begin{array}{l}\text { Percentage of } \\
\text { Distraction } \\
\text { Incidents }\end{array}$ & $\begin{array}{l}\text { Combined } \\
\text { Rank of } \\
\text { Distraction } \\
\text { Incidents }\end{array}$ \\
\hline Talking/listening on cell phone & 10 & $21.7 \%$ & 1 \\
\hline Passenger in adjacent seat & 6 & $13.0 \%$ & 2 \\
\hline Dialing hand-held cell phone & 4 & $8.7 \%$ & 3 \\
\hline Looking out center mirror & 3 & $6.5 \%$ & 4.5 \\
\hline Looking out left window & 3 & $6.5 \%$ & 4.5 \\
\hline Other external distraction & 2 & $4.3 \%$ & 9 \\
\hline Adjusting radio & 2 & $4.3 \%$ & 9 \\
\hline Cognitive - Other & 2 & $4.3 \%$ & 9 \\
\hline Combing or fixing hair & 2 & $4.3 \%$ & 9 \\
\hline Lost in thought & 2 & $4.3 \%$ & 9 \\
\hline Smoking cigar/cigarette & 2 & $4.3 \%$ & 9 \\
\hline Talking/singing/dancing (not on cell phone) & 2 & $4.3 \%$ & 9 \\
\hline Eating with utensils & 1 & $2.2 \%$ & 15.5 \\
\hline Lighting cigar/cigarette & 1 & $2.2 \%$ & 15.5 \\
\hline Operating PDA & 1 & $2.2 \%$ & 15.5 \\
\hline Reaching for object (not cell phone) & 1 & $2.2 \%$ & 15.5 \\
\hline Reading & 1 & $2.2 \%$ & 15.5 \\
\hline Looking out right window & 1 & $2.2 \%$ & 15.5 \\
\hline
\end{tabular}




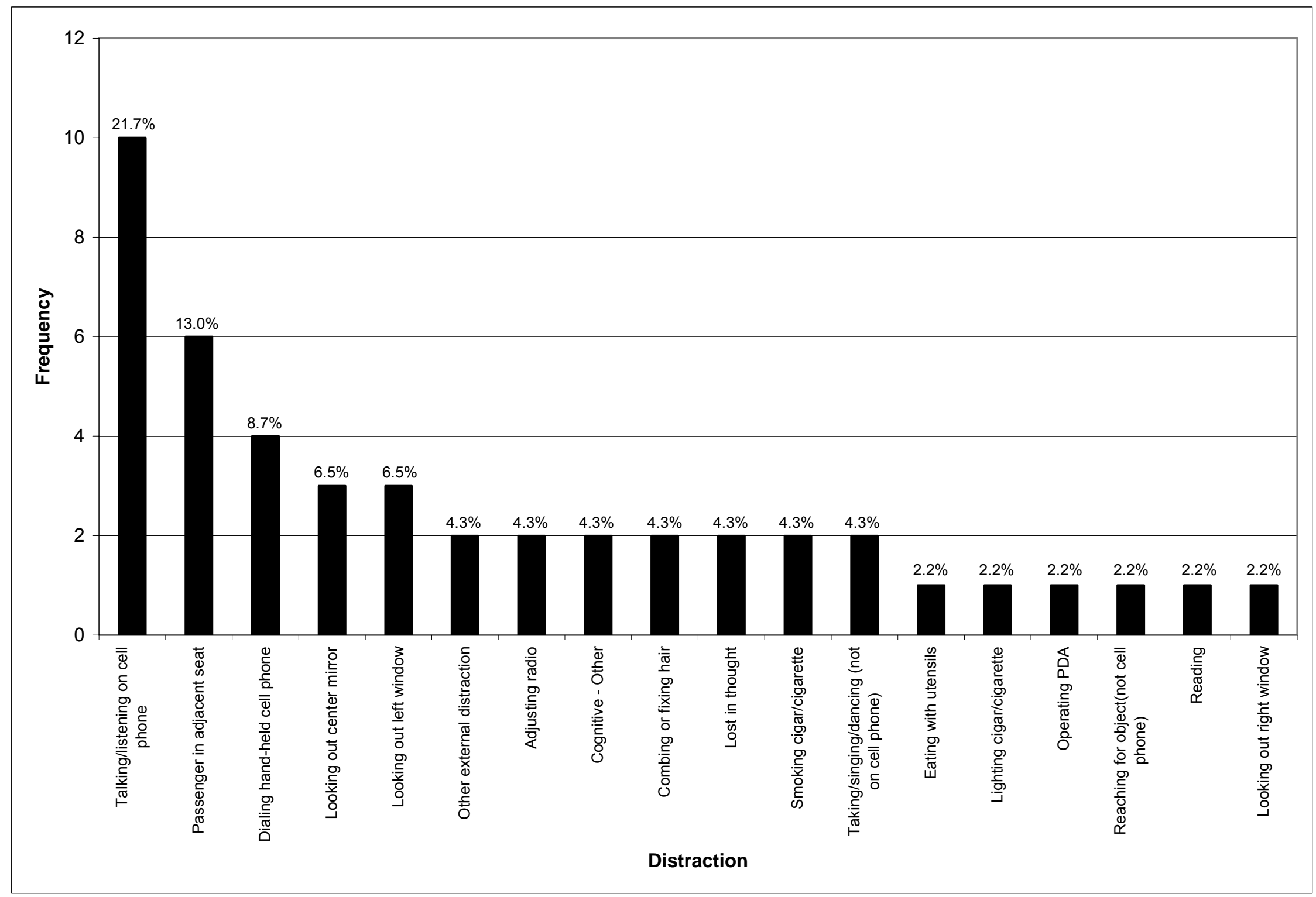

Figure 27. Frequency of the Distraction Contributing Factor in Each Sub-Category. 
Table 23 shows the frequency, percentage, and rank ordering for each sub-category in the Distraction Contributing Factor for HV driver at-fault incidents. As can be seen in Table 23, the most frequent Distractions for HV at-fault incidents were Talking/Listening on Cell Phone (22.2\%), Combing or Fixing Hair (22.2\%), Dialing Hand-Held Cell Phone (22.2\%), and Passenger in Adjacent Seat (22.2\%).

Table 23. Frequency, Percentage, and Rank Ordering of Each Sub-Category in the Distraction Contributing Factor for the HV At-Fault Incidents $(n=9)$.

\begin{tabular}{|l|c|c|c|}
\hline \multicolumn{1}{|c|}{ Distraction } & $\begin{array}{c}\text { Frequency of } \\
\text { Distractions in } \\
\text { HV At-Fault } \\
\text { Incidents }\end{array}$ & $\begin{array}{c}\text { Percentage of } \\
\text { Distractions in } \\
\text { HV At-Fault } \\
\text { Incidents }\end{array}$ & $\begin{array}{c}\text { Combined } \\
\text { Rank of } \\
\text { Distractions in } \\
\text { HV At-Fault } \\
\text { Incidents }\end{array}$ \\
\hline Talking/listening on cell phone & 2 & $22.2 \%$ & 2.5 \\
\hline Combing or fixing hair & 2 & $22.2 \%$ & 2.5 \\
\hline Dialing hand-held cell phone & 2 & $22.2 \%$ & 2.5 \\
\hline Passenger in adjacent seat & 2 & $22.2 \%$ & 2.5 \\
\hline Lost in thought & 1 & $11.1 \%$ & 5 \\
\hline
\end{tabular}

Table 24 shows the frequency, percentage, and rank ordering for each sub-category in the Distraction Contributing Factor for LV driver at-fault incidents. As can be seen in the table, the most frequent Distractions for LV at-fault incidents were Talking/Listening on Cell Phone (19.4\%) and Passenger in Adjacent Seat (12.9\%). 
Table 24. Frequency, Percentage, and Rank Ordering of Each Sub-Category in the Distraction Contributing Factor for the LV At-Fault Incidents $(n=31)$.

\begin{tabular}{|l|c|c|c|}
\hline \multicolumn{1}{|c|}{ Distraction } & $\begin{array}{c}\text { Frequency of } \\
\text { Distractions in } \\
\text { LV At-Fault } \\
\text { Incidents }\end{array}$ & $\begin{array}{c}\text { Percentage of } \\
\text { Distractions in } \\
\text { LV At-Fault } \\
\text { Incidents }\end{array}$ & $\begin{array}{c}\text { Combined } \\
\text { Rank of } \\
\text { Distractions in } \\
\text { LV At-Fault } \\
\text { Incidents }\end{array}$ \\
\hline Talking/listening on cell phone & 6 & $19.4 \%$ & 1 \\
\hline Passenger in adjacent seat & 4 & $12.9 \%$ & 2 \\
\hline Looking out center mirror & 3 & $9.7 \%$ & 3.5 \\
\hline Looking out left window & 3 & $9.7 \%$ & 3.5 \\
\hline Other external distraction & 2 & $6.5 \%$ & 7 \\
\hline Dialing hand-held cell phone & 2 & $6.5 \%$ & 7 \\
\hline Adjusting radio & 2 & $6.5 \%$ & 7 \\
\hline Smoking cigar/cigarette & 2 & $6.5 \%$ & 7 \\
\hline Cognitive - Other & 2 & $6.5 \%$ & 7 \\
\hline Lost in thought & 1 & $3.2 \%$ & 12 \\
\hline Eating with utensils & 1 & $3.2 \%$ & 12 \\
\hline Lighting cigar/cigarette & 1 & $3.2 \%$ & 12 \\
\hline Reaching for object (not cell phone) & 1 & $3.2 \%$ & $12 \%$ \\
\hline Talking/singing/dancing (not on phone) & 1 & $3.2 \%$ & 7 \\
\hline
\end{tabular}

Table 25 shows the frequency, percentage, and rank ordering for each sub-category in the Distraction Contributing Factor for the Unknown driver at-fault incidents. As can be seen in Table 25, the most frequent Distraction for Unknown at-fault incidents was Talking/Listening on Cell Phone (33.3\%).

Table 25. Frequency, Percentage, and Rank Ordering of Each Sub-Category in the Distraction Contributing Factor for the Unknown At-Fault Incidents $(n=6)$.

\begin{tabular}{|l|c|c|c|}
\hline \multicolumn{1}{|c|}{ Distraction } & $\begin{array}{c}\text { Frequency of } \\
\text { Distractions in } \\
\text { Unknown At- } \\
\text { Fault Incidents }\end{array}$ & $\begin{array}{c}\text { Percentage of } \\
\text { Distractions in } \\
\text { Unknown At- } \\
\text { Fault Incidents }\end{array}$ & $\begin{array}{c}\text { Combined } \\
\text { Rank of } \\
\text { Distractions in } \\
\text { Unknown At- } \\
\text { Fault Incidents }\end{array}$ \\
\hline Talking/listening on cell phone & 2 & $33.3 \%$ & 1 \\
\hline Talking/singing/dancing & 1 & $16.7 \%$ & 3.5 \\
\hline Operating PDA & 1 & $16.7 \%$ & 3.5 \\
\hline Reading & 1 & $16.7 \%$ & 3.5 \\
\hline Right window & 1 & $16.7 \%$ & 3.5 \\
\hline
\end{tabular}




\section{Summary of Driver Distraction}

Overall, Distraction was cited in 18.7\% of the LV-HV interaction incidents (see Figure 17). This is higher than what Stutts et al. (2003) found when they reviewed the U.S. Crashworthiness Data System (CDS) from 1995-1999. Stutts et al. (2003) found that 8.3\% of drivers involved in a fatal LV-HV interaction crash were identified as "Distracted" in the CDS database. Similarly, Stuster (1999) found that $8.7 \%$ of the LV drivers were cited with the driver-related factor "Driving Inattentively." It should be noted that Stuster (1999) only listed the twelve most frequent driverrelated factors. Thus, the percentage noted may be incomplete as it only refers to driving inattentively. Hanowski, Olson, Perez, and Dingus (2001) assessed the occurrence of "driver distraction" as a contributing factor in a naturalistic study using long-haul drivers. A total of 2,737 critical incidents were recorded in the Sleeper Berth study. Of these, 178 (6.5\%) had "driver distraction" as a contributing factor. As such, there was a considerably higher percentage of "driver distraction" related incidents in the current study as compared to previous studies.

As seen in Figure 17, the Distraction Contributing Factor was divided into discrete subcategories. When Stutts et al. (2003) assessed the CDS, they also divided the $8.3 \%$ of the "Distraction" crashes into more discrete sub-categories. They found that the most frequent distracter in the CDS involved an External Distraction (29.4\%), followed by Adjusting Radio/Cassette/CD (11.4\%) and Other Occupant (10.9\%), respectively. The current research found very different results. For example, as shown in Table 24, External Distractions (summing Left Window, Right Window, Center Mirror, and Other External Distraction) accounted for only 19.5\% of the distraction incidents for at-fault LV drivers. ${ }^{5}$ Furthermore, Adjusting the Radio and Passenger in Adjacent Seat only accounted for $6.5 \%$ and $12.9 \%$ of the distraction incidents for LV at-fault drivers, respectively.

The most frequent sub-category for the Distraction Contributing Factor for LV at-fault incidents in current research was Talking/Listening on Cell Phone (19.4\%), followed by Passenger in Adjacent Seat (12.9\%) and Looking Out Center Mirror (9.7\%), and Looking Out Left Window (9.7\%). Stutts et al. (2003) found that Using/Dialing Phone was cited in only 1.5\% of the distraction crashes, while Other Occupant (similar to Passenger in Adjacent Seat in the current research) was cited in $10.9 \%$ of the distraction crashes. Note that Stutts et al. (2003) include both talking/listening and dialing phones in their definition of phone use.

Again, these differences might highlight the discrepancy between crashes and near crashes and/or the methodologies used to obtain the data. Another likely reason for the discrepancy is that the Stutts et al. study used crash data from 1995-1999. There is little doubt that the number of cell phones in use has increased substantially from the time period of 1995-1999, as compared to 2003-2004, when the current data were collected. In addition, in many states, PARs have not been developed to account for cell phone use (Wierwille et al., 2002) and this may have also contributed to the lower percentage found in the Stutts et al. (2003) research.

\footnotetext{
${ }^{5}$ Only LV at-fault incidents were considered because only the LV drivers had instrumented vehicles.
} 


\section{CLASSIFICATION STRATEGY USED IN THE LTCCS}

\section{Accident Types}

Each of the $246 \mathrm{LV}-\mathrm{HV}$ interactions were grouped by Accident Type based on the methodology used in the LTCCS (Thieriez, Radja, and Toth, 2002). Note that there was only one LV-HV crash recorded in the 100-Car Study. Therefore, using the Accident Types from the LTCCS does not reflect an absolute match, but rather a relative match. However, to facilitate future data comparisons with the near-crash data collected in the current study with other studies using the LTCCS, each of the 246 LV-HV interactions were coded using the LTCCS classification scheme. Because only one crash occurred, the closest match with respect to Accident Types was recorded for each incident. Table 26 shows the LTCCS Accident Type descriptions. 
Table 26. Description of the LTCCS Accident Types (Thieriez, Radja, and Toth, 2002).

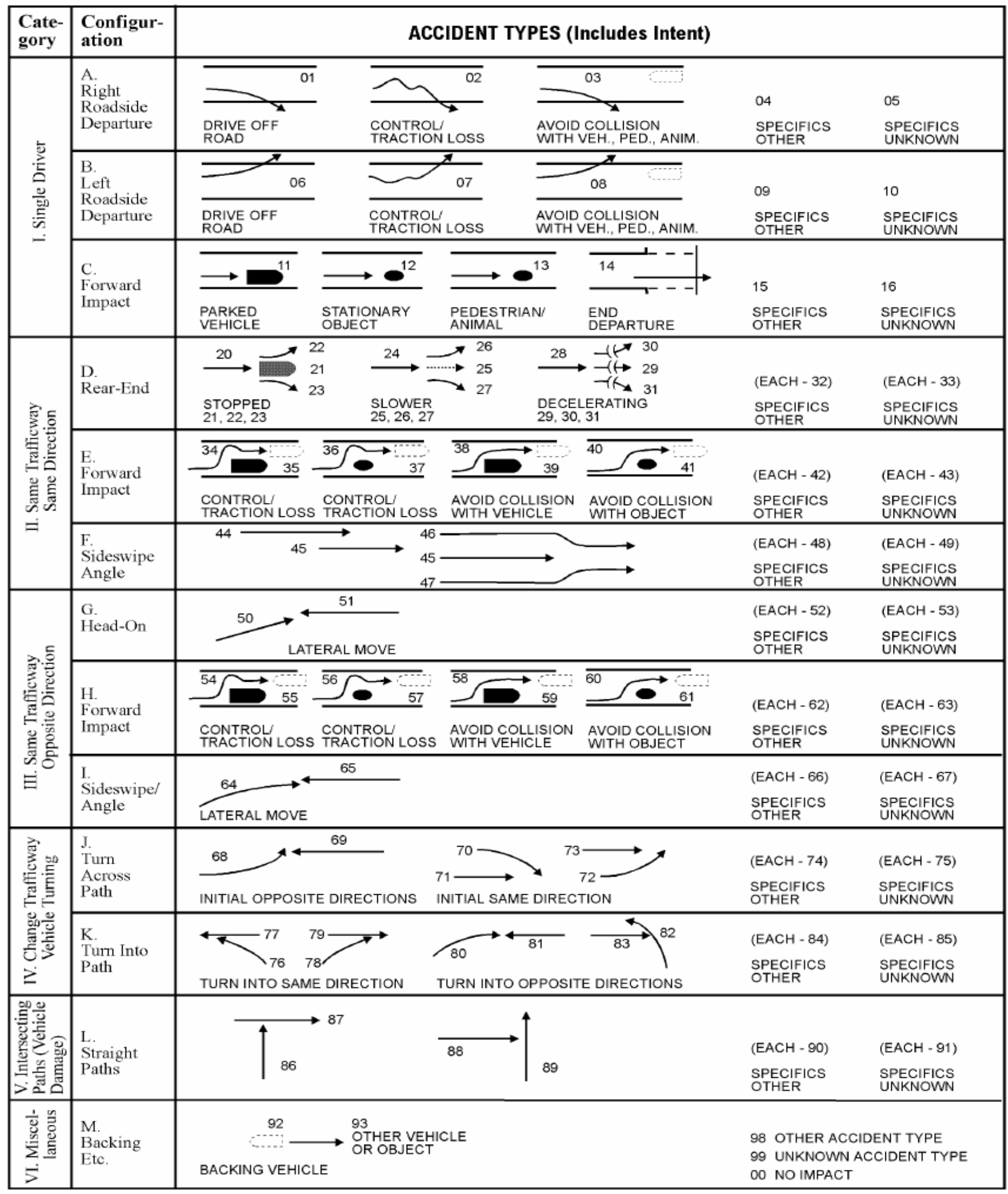


Table 27 shows the frequency, percentage, and rank ordering for the Accident Types across the entire data set. As can be seen, the most commonly occurring Accident Type that involved an interaction between a LV and HV was Scenario 38/39: Same Trafficway Same Direction, Forward Impact, Avoid Collision With Vehicle. Again, it is important to note that the Accident Types listed above in Table 26 were intended to be used for crashes and not for near crashes. Since this was the case, a "what if" approach was taken where data analysts coded the Accident Type to reflect what would likely have occurred had there been a crash. Thus, because of this subjective interpretation, not all events fit neatly into a category. As such, the results from the Accident Type categorization are not as “clean” as those from the Incident Type categories used in Hanowski, Keisler, and Wierwille (2004). Figure 28 shows a bar graph of the 246 incidents as a function of Accident Type.

Table 27. Frequency, Percentage, and Rank Ordering of the LTCCS Accident Types Across the Entire Data Set $\left(\mathbf{N}_{\text {Total }}=\mathbf{2 4 6}\right)$.

\begin{tabular}{|c|c|c|c|c|c|}
\hline $\begin{array}{l}\text { LV } \\
\text { Accident } \\
\text { Type }\end{array}$ & $\begin{array}{c}\text { HV } \\
\text { Accident } \\
\text { Type }\end{array}$ & Accident Type Description & $\begin{array}{c}\text { Frequency } \\
\text { of Accident } \\
\text { Types } \\
\left(N_{\text {Total }}=246\right)\end{array}$ & $\begin{array}{c}\text { Percentage } \\
\text { of Accident } \\
\text { Types } \\
\left(\mathrm{N}_{\text {Total }}=246\right)\end{array}$ & $\begin{array}{c}\text { Combined } \\
\text { Rank of } \\
\text { Accident } \\
\text { Types }\end{array}$ \\
\hline 38 & 39 & $\begin{array}{l}\text { Same Trafficway/Same Direction: Forward } \\
\text { Impact: Avoid Collision with Vehicle }\end{array}$ & 49 & $19.9 \%$ & 1 \\
\hline 20 & 21 & $\begin{array}{l}\text { Same Trafficway/Same Direction: Rear- } \\
\text { End: Approaches Stopped Vehicle }\end{array}$ & 40 & $16.3 \%$ & 2 \\
\hline 28 & 29 & $\begin{array}{l}\text { Same Trafficway/Same Direction: Rear- } \\
\text { End: Approaches Decelerating Vehicle }\end{array}$ & 32 & $13.0 \%$ & 3 \\
\hline 44 & 45 & $\begin{array}{l}\text { Same Trafficway/Same Direction: } \\
\text { Sideswipe Angle: In Blind Spot }\end{array}$ & 26 & $10.6 \%$ & 4 \\
\hline 24 & 25 & $\begin{array}{l}\text { Same Trafficway/Same Direction: Rear- } \\
\text { End: Approaches Slower Constant Speed }\end{array}$ & 16 & $6.5 \%$ & 5 \\
\hline 47 & 45 & $\begin{array}{l}\text { Same Trafficway/Same Direction: } \\
\text { Sideswipe Angle: Side Cutoff on the Right }\end{array}$ & 10 & $4.1 \%$ & 6 \\
\hline 46 & 45 & $\begin{array}{l}\text { Same Trafficway/Same Direction: } \\
\text { Sideswipe Angle: Side Cutoff on the Left }\end{array}$ & 9 & $3.7 \%$ & 7 \\
\hline 45 & 44 & $\begin{array}{l}\text { Same Trafficway/Same Direction: } \\
\text { Sideswipe Angle: Has Vehicle in their Blind }\end{array}$ & 7 & $2.8 \%$ & 8 \\
\hline 83 & 82 & $\begin{array}{l}\text { Change Trafficway/Opposite Direction: Turn } \\
\text { Into Path: Turn Into Opposite Direction }\end{array}$ & 6 & $2.4 \%$ & 9 \\
\hline 25 & 24 & $\begin{array}{l}\text { Same Trafficway/Same Direction: Rear- } \\
\text { End: Slower Constant Speed }\end{array}$ & 5 & $2.0 \%$ & 11 \\
\hline 52 & 52 & $\begin{array}{l}\text { Same Trafficway/Opposite Direction: Head- } \\
\text { On: Other }\end{array}$ & 5 & $2.0 \%$ & 11 \\
\hline 58 & 59 & $\begin{array}{l}\text { Same Trafficway/Opposite Direction: } \\
\text { Forward Impact: Avoid Collision with }\end{array}$ & 5 & $2.0 \%$ & 11 \\
\hline 45 & 47 & $\begin{array}{l}\text { Same Trafficway/Same Direction: } \\
\text { Sideswipe Angle: Side Cutoff on the Right }\end{array}$ & 4 & $1.6 \%$ & 13.5 \\
\hline 69 & 68 & $\begin{array}{l}\text { Change Trafficway/Vehicle Turning: Turn } \\
\text { Across Path: Initial Opposite Directions }\end{array}$ & 4 & $1.6 \%$ & 13.5 \\
\hline 21 & 20 & $\begin{array}{l}\text { Same Trafficway/Same Direction: Rear- } \\
\text { End: Stopped }\end{array}$ & 3 & $1.2 \%$ & 16.5 \\
\hline 29 & 28 & $\begin{array}{l}\text { Same Trafficway/Same Direction: Rear- } \\
\text { End: Decelerating }\end{array}$ & 3 & $1.2 \%$ & 16.5 \\
\hline
\end{tabular}




\begin{tabular}{|c|c|c|c|c|c|}
\hline $\begin{array}{l}\text { LV } \\
\text { Accident } \\
\text { Type }\end{array}$ & $\begin{array}{l}\text { HV } \\
\text { Accident } \\
\text { Type }\end{array}$ & Accident Type Description & $\begin{array}{l}\text { Frequency } \\
\text { of Accident } \\
\text { Types } \\
\left(\mathrm{N}_{\text {Total }}=246\right)\end{array}$ & $\begin{array}{c}\text { Percentage } \\
\text { of Accident } \\
\text { Types } \\
\left(N_{\text {Total }}=246\right)\end{array}$ & $\begin{array}{l}\text { Combined } \\
\text { Rank of } \\
\text { Accident } \\
\text { Types }\end{array}$ \\
\hline 45 & 46 & $\begin{array}{l}\text { Same Trafficway/Same Direction: } \\
\text { Sideswipe Angle: Side Cutoff on the Left }\end{array}$ & 3 & $1.2 \%$ & 16.5 \\
\hline 98 & 98 & Other Accident Type & 3 & $1.2 \%$ & 16.5 \\
\hline 11 & 11 & $\begin{array}{l}\text { Single Driver: Forward Impact: Parked } \\
\text { Vehicle }\end{array}$ & 2 & $0.8 \%$ & 20 \\
\hline 79 & 78 & $\begin{array}{l}\text { Change Trafficway/Vehicle Turning: Turn } \\
\text { Into Path: Turn Into Same Direction }\end{array}$ & 2 & $0.8 \%$ & 20 \\
\hline 86 & 87 & $\begin{array}{l}\text { Intersecting Paths: Straight Paths: Impact } \\
\text { on Right Side }\end{array}$ & 2 & $0.8 \%$ & 20 \\
\hline 28 & 30 & $\begin{array}{l}\text { Same Trafficway/Same Direction: Rear- } \\
\text { End: Approaches Decelerating Vehicle } \\
\text { Turning Left }\end{array}$ & 1 & $0.4 \%$ & 26.5 \\
\hline 42 & 42 & $\begin{array}{l}\text { Same Trafficway/Same Direction: Forward } \\
\text { Impact: Other }\end{array}$ & 1 & $0.4 \%$ & 26.5 \\
\hline 50 & 51 & $\begin{array}{l}\text { Same Trafficway/Opposite Direction: Head- } \\
\text { On: Lateral Move }\end{array}$ & 1 & $0.4 \%$ & 26.5 \\
\hline 71 & 70 & $\begin{array}{l}\text { Change Trafficway/Vehicle Turning: Turn } \\
\text { Across Path: Initial Same Directions }\end{array}$ & 1 & $0.4 \%$ & 26.5 \\
\hline 75 & 75 & $\begin{array}{l}\text { Change Trafficway/Vehicle Turning: Turn } \\
\text { Across Path: Unknown }\end{array}$ & 1 & $0.4 \%$ & 26.5 \\
\hline 76 & 77 & $\begin{array}{l}\text { Change Trafficway/Vehicle Turning: Turn } \\
\text { Into Path: Turn Into Same Direction }\end{array}$ & 1 & $0.4 \%$ & 26.5 \\
\hline 77 & 76 & $\begin{array}{l}\text { Change Trafficway/Vehicle Turning: Turn } \\
\text { Into Path: Turn Into Same Direction }\end{array}$ & 1 & $0.4 \%$ & 26.5 \\
\hline 81 & 80 & $\begin{array}{l}\text { Change Trafficway/Vehicle Turning: Turn } \\
\text { Into Path: Turn Into Opposite Direction }\end{array}$ & 1 & $0.4 \%$ & 26.5 \\
\hline 88 & 89 & $\begin{array}{l}\text { Intersecting Paths: Straight Paths: Impact } \\
\text { on Left Side }\end{array}$ & 1 & $0.4 \%$ & 26.5 \\
\hline 65 & 64 & $\begin{array}{l}\text { Same Trafficway/Opposite Direction: } \\
\text { Sideswipe Angle: Lateral Move }\end{array}$ & 1 & $0.4 \%$ & 26.5 \\
\hline
\end{tabular}




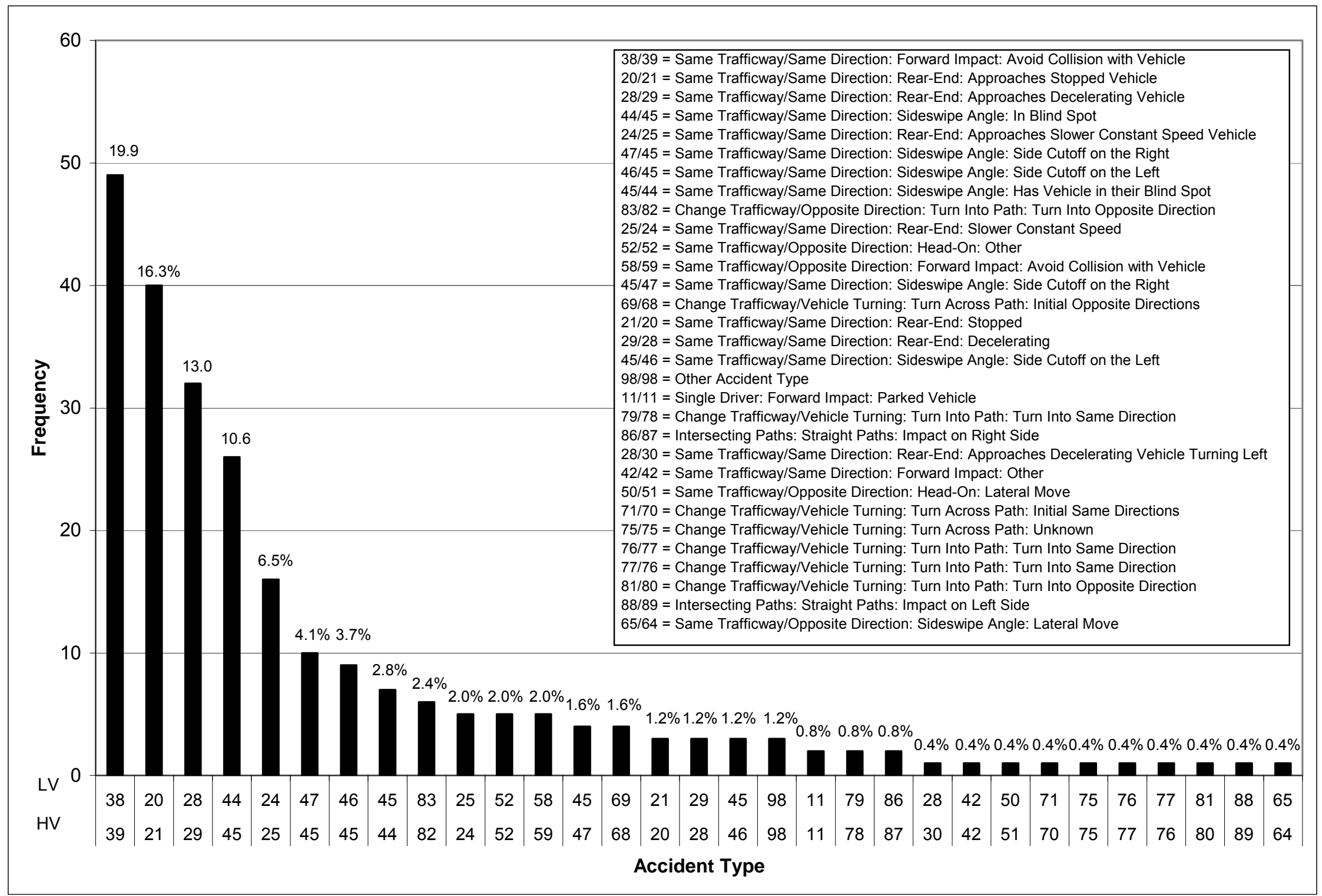

Figure 28. Frequency of Accident Types Across the Entire Data Set $\left(\mathbf{N}_{\text {Total }}=\mathbf{2 4 6}\right)$. 
Table 28 illustrates the frequency, percentage, and rank ordering of the Accident Types for HV driver at-fault incidents. The most frequent Accident Type for the HV driver at-fault incidents was Scenario 44/45: Same Trafficway/Same Direction: Sideswipe Angle: In Blind Spot (27.8\%), followed by Scenarios 38/39: Same Trafficway/Same Direction: Forward Impact: Avoid Collision with Vehicle (15.2\%) and 25/25: Same Trafficway/Same Direction: Rear-End: Approaches Slower Constant Speed Vehicle (8.9\%). Figure 29 shows a bar graph of the 79 HV driver at-fault incidents as a function of the Accident Type. 
Table 28. Frequency, Percentage, and Rank Ordering of the Accident Types for HV Driver At-Fault Incidents $\left(\mathbf{n}_{\mathbf{H V}}=\mathbf{7 9}\right)$.

\begin{tabular}{|c|c|c|c|c|c|}
\hline $\begin{array}{l}\text { LV } \\
\text { Accident } \\
\text { Type }\end{array}$ & $\begin{array}{c}\text { HV } \\
\text { Accident } \\
\text { Type }\end{array}$ & Accident Type Description & $\begin{array}{l}\text { Frequency } \\
\text { of HV Driver } \\
\text { At-Fault } \\
\text { Incidents } \\
\left(n_{\mathrm{HV}}=79\right)\end{array}$ & $\begin{array}{c}\text { Percentage } \\
\text { of HV } \\
\text { Driver At- } \\
\text { Fault } \\
\text { Incidents } \\
\left(\mathrm{n}_{\mathrm{HV}}=79\right) \\
\end{array}$ & $\begin{array}{l}\text { Combined } \\
\text { Rank of } \\
\text { HV Driver } \\
\text { At-Fault } \\
\text { Incidents }\end{array}$ \\
\hline 44 & 45 & $\begin{array}{l}\text { Same Trafficway/Same Direction: Sideswipe } \\
\text { Angle: In Blind Spot }\end{array}$ & 22 & $27.8 \%$ & 1 \\
\hline 38 & 39 & $\begin{array}{l}\text { Same Trafficway/Same Direction: Forward } \\
\text { Impact: Avoid Collision with Vehicle }\end{array}$ & 12 & $15.2 \%$ & 2 \\
\hline 24 & 25 & $\begin{array}{l}\text { Same Trafficway/Same Direction: Rear-End: } \\
\text { Approaches Slower Constant Speed Vehicle }\end{array}$ & 7 & $8.9 \%$ & 3 \\
\hline 83 & 82 & $\begin{array}{l}\text { Change Trafficway/Opposite Direction: Turn } \\
\text { Into Path: Turn Into Opposite Direction }\end{array}$ & 5 & $6.3 \%$ & 4 \\
\hline 45 & 44 & $\begin{array}{l}\text { Same Trafficway/Same Direction: Sideswipe } \\
\text { Angle: Has Vehicle in their Blind Spot }\end{array}$ & 4 & $5.1 \%$ & 5.5 \\
\hline 69 & 68 & $\begin{array}{l}\text { Change Trafficway/Vehicle Turning: Turn } \\
\text { Across Path: Initial Opposite Directions }\end{array}$ & 4 & $5.1 \%$ & 5.5 \\
\hline 28 & 29 & $\begin{array}{l}\text { Same Trafficway/Same Direction: Rear-End: } \\
\text { Approaches Decelerating Vehicle }\end{array}$ & 3 & $3.8 \%$ & 7.5 \\
\hline 45 & 46 & $\begin{array}{l}\text { Same Trafficway/Same Direction: Sideswipe } \\
\text { Angle: Side Cutoff on the Left }\end{array}$ & 3 & $3.8 \%$ & 7.5 \\
\hline 20 & 21 & $\begin{array}{l}\text { Same Trafficway/Same Direction: Rear-End: } \\
\text { Approaches Stopped Vehicle }\end{array}$ & 2 & $2.5 \%$ & 10 \\
\hline 45 & 47 & $\begin{array}{l}\text { Same Trafficway/Same Direction: Sideswipe } \\
\text { Angle: Side Cutoff on the Right }\end{array}$ & 2 & $2.5 \%$ & 10 \\
\hline 46 & 45 & $\begin{array}{l}\text { Same Trafficway/Same Direction: Sideswipe } \\
\text { Angle: Side Cutoff on the Left }\end{array}$ & 2 & $2.5 \%$ & 10 \\
\hline 79 & 78 & $\begin{array}{l}\text { Change Trafficway/Vehicle Turning: Turn } \\
\text { Into Path: Turn Into Same Direction }\end{array}$ & 2 & $2.5 \%$ & 10 \\
\hline 11 & 11 & $\begin{array}{l}\text { Single Driver: Forward Impact: Parked } \\
\text { Vehicle }\end{array}$ & 1 & $1.3 \%$ & 18 \\
\hline 21 & 20 & $\begin{array}{l}\text { Same Trafficway/Same Direction: Rear-End: } \\
\text { Stopped }\end{array}$ & 1 & $1.3 \%$ & 18 \\
\hline 29 & 28 & $\begin{array}{l}\text { Same Trafficway/Same Direction: Rear-End: } \\
\text { Decelerating }\end{array}$ & 1 & $1.3 \%$ & 18 \\
\hline 50 & 51 & $\begin{array}{l}\text { Same Trafficway/Opposite Direction: Head- } \\
\text { On: Lateral Move }\end{array}$ & 1 & $1.3 \%$ & 18 \\
\hline 58 & 59 & $\begin{array}{l}\text { Same Trafficway/Opposite Direction: } \\
\text { Forward Impact: Avoid Collision with Vehicle }\end{array}$ & 1 & $1.3 \%$ & 18 \\
\hline 71 & 70 & $\begin{array}{l}\text { Change Trafficway/Vehicle Turning: Turn } \\
\text { Across Path: Initial Same Directions }\end{array}$ & 1 & $1.3 \%$ & 18 \\
\hline 77 & 76 & $\begin{array}{l}\text { Change Trafficway/Vehicle Turning: Turn } \\
\text { Into Path: Turn Into Same Direction }\end{array}$ & 1 & $1.3 \%$ & 18 \\
\hline 81 & 80 & $\begin{array}{l}\text { Change Trafficway/Vehicle Turning: Turn } \\
\text { Into Path: Turn Into Opposite Direction }\end{array}$ & 1 & $1.3 \%$ & 18 \\
\hline 86 & 87 & $\begin{array}{l}\text { Intersecting Paths: Straight Paths: Impact on } \\
\text { Right Side }\end{array}$ & 1 & $1.3 \%$ & 18 \\
\hline 88 & 89 & $\begin{array}{l}\text { Intersecting Paths: Straight Paths: Impact on } \\
\text { Left Side }\end{array}$ & 1 & $1.3 \%$ & 18 \\
\hline 98 & 98 & Other Accident Type & 1 & $1.3 \%$ & 18 \\
\hline
\end{tabular}




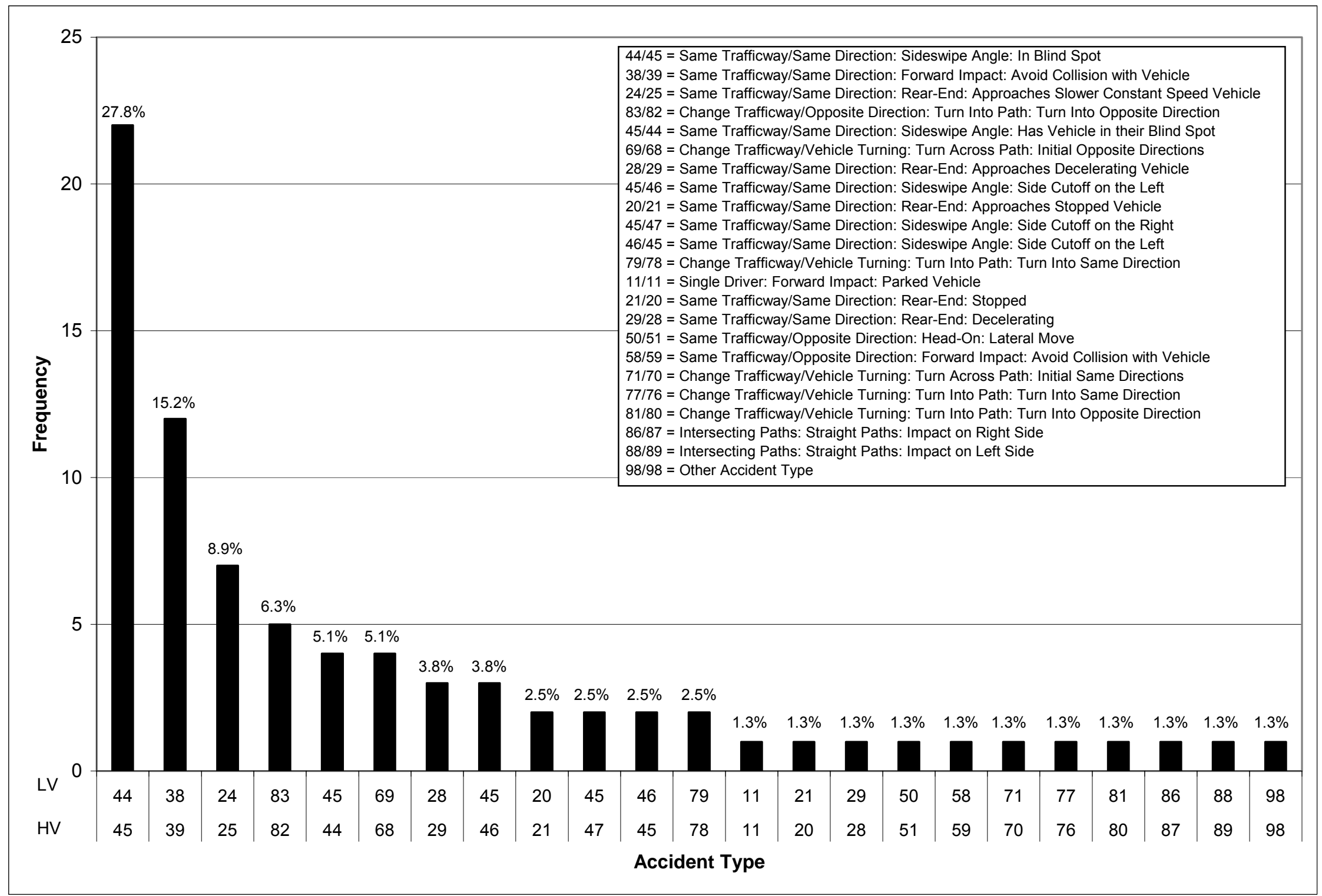

Figure 29. Frequency of Accident Types for HV Driver At-Fault Incidents $\left(\mathbf{n}_{\mathrm{HV}}=\mathbf{7 9}\right)$. 
Table 29 illustrates the frequency, percentage, and rank ordering of the Accident Types for LV driver at-fault incidents. The most frequent Accident Types for HV driver at-fault incidents was Scenario 20/21: Same Trafficway/Same Direction: Rear-End: Approaches Stopped Vehicle (26.8\%), followed by Scenarios 38/39: Same Trafficway/Same Direction: Forward Impact: Avoid Collision with Vehicle (22.5\%) and 28/29: Same Trafficway/Same Direction: Rear-End: Approaches Decelerating Vehicle (17.4\%). Figure 30 shows a bar graph of the 138 LV driver atfault incidents as a function of the Accident Type. 
Table 29. Frequency, Percentage, and Rank Ordering of the Accident Types for LV Driver At-Fault Incidents $\left(n_{L V}=138\right)$.

\begin{tabular}{|c|c|c|c|c|c|}
\hline $\begin{array}{c}\text { LV } \\
\text { Accident } \\
\text { Type }\end{array}$ & $\begin{array}{c}\text { HV } \\
\text { Accident } \\
\text { Type }\end{array}$ & Accident Type Description & $\begin{array}{l}\text { Frequency } \\
\text { of LV Driver } \\
\text { At-Fault } \\
\text { Incidents } \\
\left(\mathrm{n}_{\mathrm{LV}}=138\right)\end{array}$ & $\begin{array}{c}\text { Percentage } \\
\text { of LV } \\
\text { Driver At- } \\
\text { Fault } \\
\text { Incidents } \\
\left(\mathrm{n}_{\mathrm{LV}}=138\right) \\
\end{array}$ & $\begin{array}{l}\text { Combined } \\
\text { Rank of } \\
\text { LV Driver } \\
\text { At-Fault } \\
\text { Incidents }\end{array}$ \\
\hline 20 & 21 & $\begin{array}{l}\text { Same Trafficway/Same Direction: Rear-End: } \\
\text { Approaches Stopped Vehicle }\end{array}$ & 37 & $26.8 \%$ & 1 \\
\hline 38 & 39 & $\begin{array}{l}\text { Same Trafficway/Same Direction: Forward } \\
\text { Impact: Avoid Collision with Vehicle }\end{array}$ & 31 & $22.5 \%$ & 2 \\
\hline 28 & 29 & $\begin{array}{l}\text { Same Trafficway/Same Direction: Rear-End: } \\
\text { Approaches Decelerating Vehicle }\end{array}$ & 24 & $17.4 \%$ & 3 \\
\hline 47 & 45 & $\begin{array}{l}\text { Same Trafficway/Same Direction: Sideswipe } \\
\text { Angle: Side Cutoff on the Right }\end{array}$ & 10 & $7.2 \%$ & 4 \\
\hline 24 & 25 & $\begin{array}{l}\text { Same Trafficway/Same Direction: Rear-End: } \\
\text { Approaches Slower Constant Speed Vehicle }\end{array}$ & 8 & $5.8 \%$ & 5 \\
\hline 46 & 45 & $\begin{array}{l}\text { Same Trafficway/Same Direction: Sideswipe } \\
\text { Angle: Side Cutoff on the Left }\end{array}$ & 6 & $4.3 \%$ & 6 \\
\hline 25 & 24 & $\begin{array}{l}\text { Same Trafficway/Same Direction: Rear-End: } \\
\text { Slower Constant Speed }\end{array}$ & 3 & $2.2 \%$ & 8 \\
\hline 44 & 45 & $\begin{array}{l}\text { Same Trafficway/Same Direction: Sideswipe } \\
\text { Angle: In Blind Spot }\end{array}$ & 3 & $2.2 \%$ & 8 \\
\hline 58 & 59 & $\begin{array}{l}\text { Same Trafficway/Opposite Direction: } \\
\text { Forward Impact: Avoid Collision with Vehicle }\end{array}$ & 3 & $2.2 \%$ & 8 \\
\hline 29 & 28 & $\begin{array}{l}\text { Same Trafficway/Same Direction: Rear-End: } \\
\text { Decelerating }\end{array}$ & 2 & $1.4 \%$ & 11 \\
\hline 45 & 47 & $\begin{array}{l}\text { Same Trafficway/Same Direction: Sideswipe } \\
\text { Angle: Side Cutoff on the Right }\end{array}$ & 2 & $1.4 \%$ & 11 \\
\hline 52 & 52 & $\begin{array}{l}\text { Same Trafficway/Opposite Direction: Head- } \\
\text { On: Other }\end{array}$ & 2 & $1.4 \%$ & 11 \\
\hline 21 & 20 & $\begin{array}{l}\text { Same Trafficway/Same Direction: Rear-End: } \\
\text { Stopped }\end{array}$ & 1 & $0.7 \%$ & 16 \\
\hline 28 & 30 & $\begin{array}{l}\text { Same Trafficway/Same Direction: Rear-End: } \\
\text { Approaches Decelerating Vehicle Turning }\end{array}$ & 1 & $0.7 \%$ & 16 \\
\hline 45 & 44 & $\begin{array}{l}\text { Trafficway/Same Direction: Sideswipe Angle: } \\
\text { Has Vehicle in their Blind Spot }\end{array}$ & 1 & $0.7 \%$ & 16 \\
\hline 65 & 64 & $\begin{array}{l}\text { Same Trafficway/Opposite Direction: } \\
\text { Sideswipe Angle: Lateral Move }\end{array}$ & 1 & $0.7 \%$ & 16 \\
\hline 75 & 75 & $\begin{array}{l}\text { Change Trafficway/Vehicle Turning: Turn } \\
\text { Across Path: Unknown }\end{array}$ & 1 & $0.7 \%$ & 16 \\
\hline 76 & 77 & $\begin{array}{l}\text { Change Trafficway/Vehicle Turning: Turn } \\
\text { Into Path: Turn Into Same Direction }\end{array}$ & 1 & $0.7 \%$ & 16 \\
\hline 98 & 98 & Other Accident Type & 1 & $0.7 \%$ & 16 \\
\hline
\end{tabular}




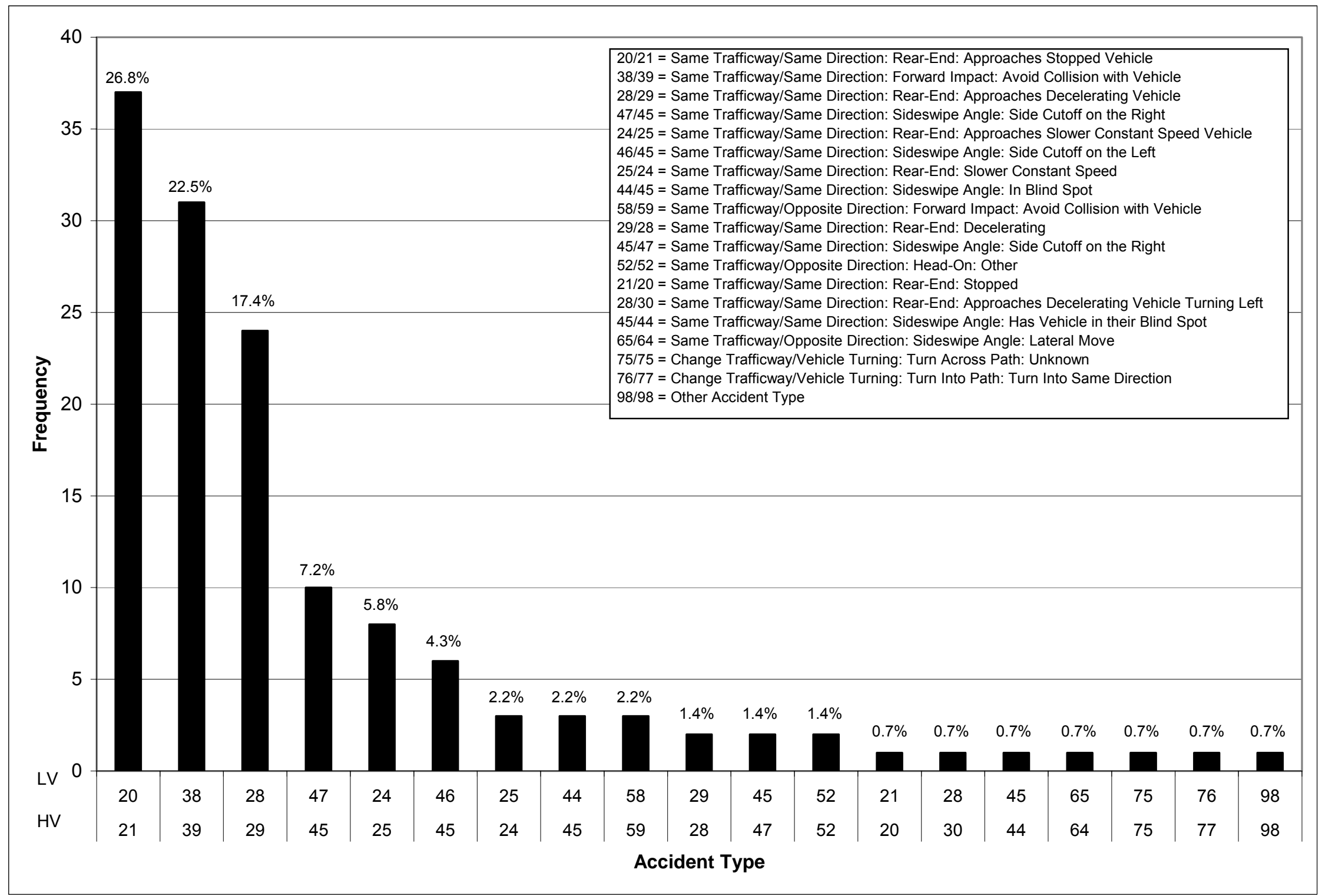

Figure 30. Frequency of Accident Types for $L V$ driver At-Fault Incidents $\left(n_{L V}=138\right)$. 
Table 30 shows the frequency, percentage, and rank ordering of the Accident Types for Unknown driver at-fault incidents. The most frequent Accident Type for the Unknown Accident Types was Scenario 38/39: Same Trafficway Same Direction; Forward Impact, Avoid Collision With Vehicle (20.7\%); followed by Scenarios 28/29: Same Trafficway/Same Direction: RearEnd: Approaches Decelerating Vehicle (17.2\%) and 52/52: Same Trafficway/Opposite Direction: Head-On: Other (10.3\%). Figure 31 shows a bar graph of the 29 Unknown at-fault incidents as a function of the Accident Type.

Table 30. Frequency, Percentage, and Rank Ordering of the Accident Types Where It was Unknown if the LV or the HV driver was At-Fault $\left(n_{U n}=29\right)$.

\begin{tabular}{|c|c|c|c|c|c|}
\hline $\begin{array}{l}\text { LV } \\
\text { Accident } \\
\text { Type }\end{array}$ & $\begin{array}{c}\text { HV } \\
\text { Accident } \\
\text { Type }\end{array}$ & Accident Type Description & $\begin{array}{l}\text { Frequency } \\
\text { of Unknown } \\
\text { At-Fault } \\
\text { Incidents } \\
\text { (nun }=29)\end{array}$ & $\begin{array}{c}\text { Percentage } \\
\text { of } \\
\text { Unknown } \\
\text { At-Fault } \\
\text { Incidents } \\
\left(n_{U n}=29\right)\end{array}$ & $\begin{array}{l}\text { Combined } \\
\text { Rank of } \\
\text { Unknown } \\
\text { At-Fault } \\
\text { Incidents }\end{array}$ \\
\hline 38 & 39 & $\begin{array}{l}\text { Same Trafficway/Same Direction: Forward } \\
\text { Impact: Avoid Collision with Vehicle }\end{array}$ & 6 & $20.7 \%$ & 1 \\
\hline 28 & 29 & $\begin{array}{l}\text { Same Trafficway/Same Direction: Rear- } \\
\text { End: Approaches Decelerating Vehicle }\end{array}$ & 5 & $17.2 \%$ & 2 \\
\hline 52 & 52 & $\begin{array}{l}\text { Same Trafficway/Opposite Direction: Head- } \\
\text { On: Other }\end{array}$ & 3 & $10.3 \%$ & 3 \\
\hline 45 & 44 & $\begin{array}{l}\text { Same Trafficway/Same Direction: } \\
\text { Sideswipe Angle: Has Vehicle in their Blind }\end{array}$ & 2 & $6.9 \%$ & 4 \\
\hline 11 & 11 & $\begin{array}{l}\text { Single Driver: Forward Impact: Parked } \\
\text { Vehicle }\end{array}$ & 1 & $3.4 \%$ & 11 \\
\hline 20 & 21 & $\begin{array}{l}\text { Same Trafficway/Same Direction: Rear- } \\
\text { End: Approaches Stopped Vehicle }\end{array}$ & 1 & $3.4 \%$ & 11 \\
\hline 21 & 20 & $\begin{array}{l}\text { Same Trafficway/Same Direction: Rear- } \\
\text { End: Stopped }\end{array}$ & 1 & $3.4 \%$ & 11 \\
\hline 24 & 25 & $\begin{array}{l}\text { Same Trafficway/Same Direction: Rear- } \\
\text { End: Approaches Slower Constant Speed }\end{array}$ & 1 & $3.4 \%$ & 11 \\
\hline 25 & 24 & $\begin{array}{l}\text { Same Trafficway/Same Direction: Rear- } \\
\text { End: Slower Constant Speed }\end{array}$ & 1 & $3.4 \%$ & 11 \\
\hline 42 & 42 & $\begin{array}{l}\text { Same Trafficway/Same Direction: Forward } \\
\text { Impact: Other }\end{array}$ & 1 & $3.4 \%$ & 11 \\
\hline 44 & 45 & $\begin{array}{l}\text { Same Trafficway/Same Direction: } \\
\text { Sideswipe Angle: In Blind Spot }\end{array}$ & 1 & $3.4 \%$ & 11 \\
\hline 46 & 45 & $\begin{array}{l}\text { Same Trafficway/Same Direction: } \\
\text { Sideswipe Angle: Side Cutoff on the Left }\end{array}$ & 1 & $3.4 \%$ & 11 \\
\hline 58 & 59 & $\begin{array}{l}\text { Same Trafficway/Opposite Direction: } \\
\text { Forward Impact: Avoid Collision with }\end{array}$ & 1 & $3.4 \%$ & 11 \\
\hline 79 & 78 & $\begin{array}{l}\text { Change Trafficway/Vehicle Turning: Turn } \\
\text { Into Path: Turn Into Same Direction }\end{array}$ & 1 & $3.4 \%$ & 11 \\
\hline 83 & 82 & $\begin{array}{l}\text { Change Trafficway/Opposite Direction: Turn } \\
\text { Into Path: Turn Into Opposite Direction }\end{array}$ & 1 & $3.4 \%$ & 11 \\
\hline 86 & 87 & $\begin{array}{l}\text { Intersecting Paths: Straight Paths: Impact } \\
\text { on Right Side }\end{array}$ & 1 & $3.4 \%$ & 11 \\
\hline 98 & 98 & Other Accident Type & 1 & $3.4 \%$ & 11 \\
\hline
\end{tabular}




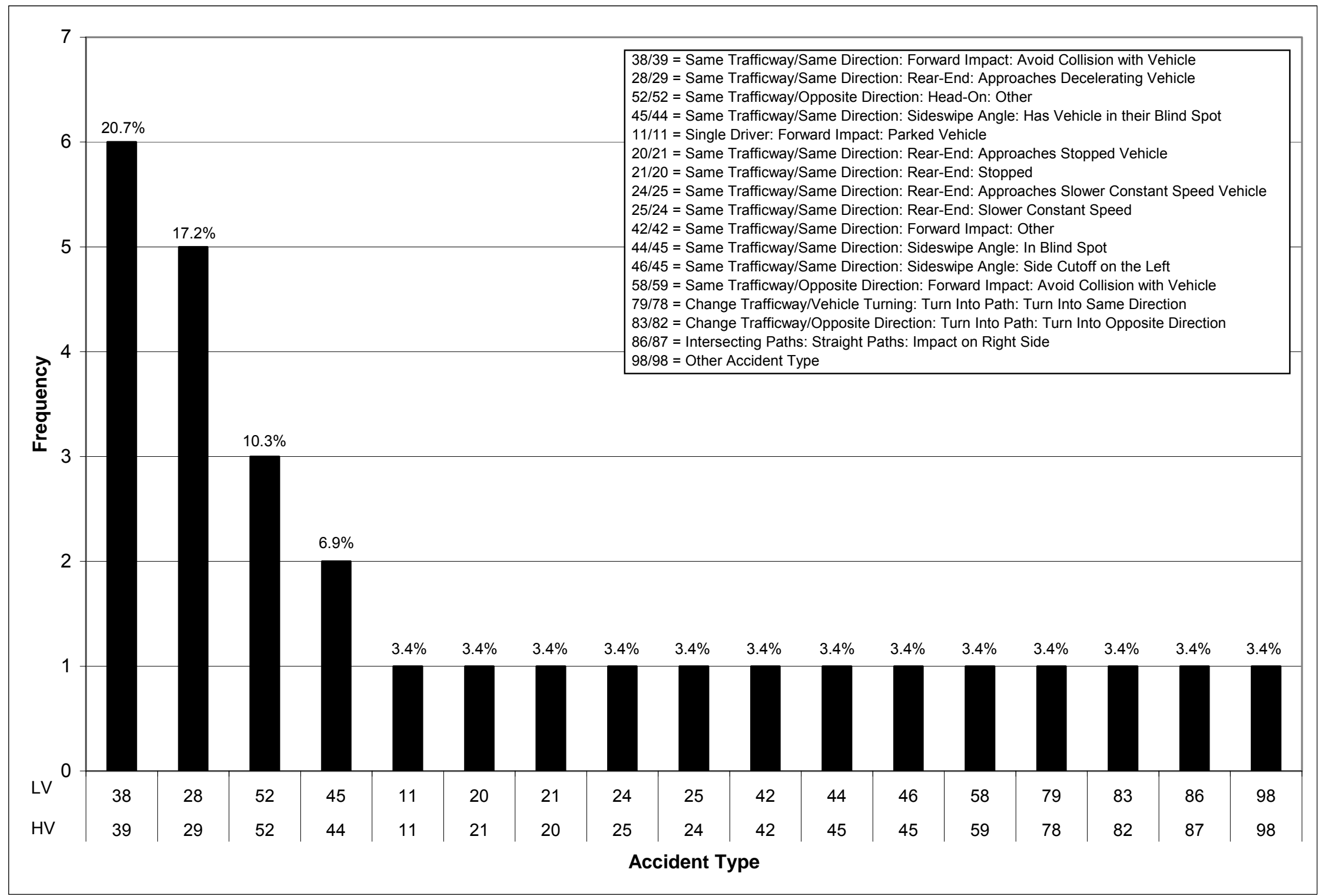

Figure 31. Frequency of Accident Types for Unknown At-Fault Incidents $\left(n_{U n}=29\right)$. 
Figure 32 shows a bar graph comparing the Accident Types, with respect to the driver that was assessed to have been at-fault, for the three groups (LV, HV, and Unknown). The figure shows that the Accident Types varied depending on whether the HV or LV driver was at fault. The most commonly occurring Accident Types were Scenarios 38/39: Same Trafficway/Same Direction: Forward Impact: Avoid Collision with Vehicle; 20/21: Same Trafficway/Same Direction: Rear-End: Approaches Stopped Vehicle; 28/29: Same Trafficway/Same Direction: Rear-End: Approaches Decelerating Vehicle. 


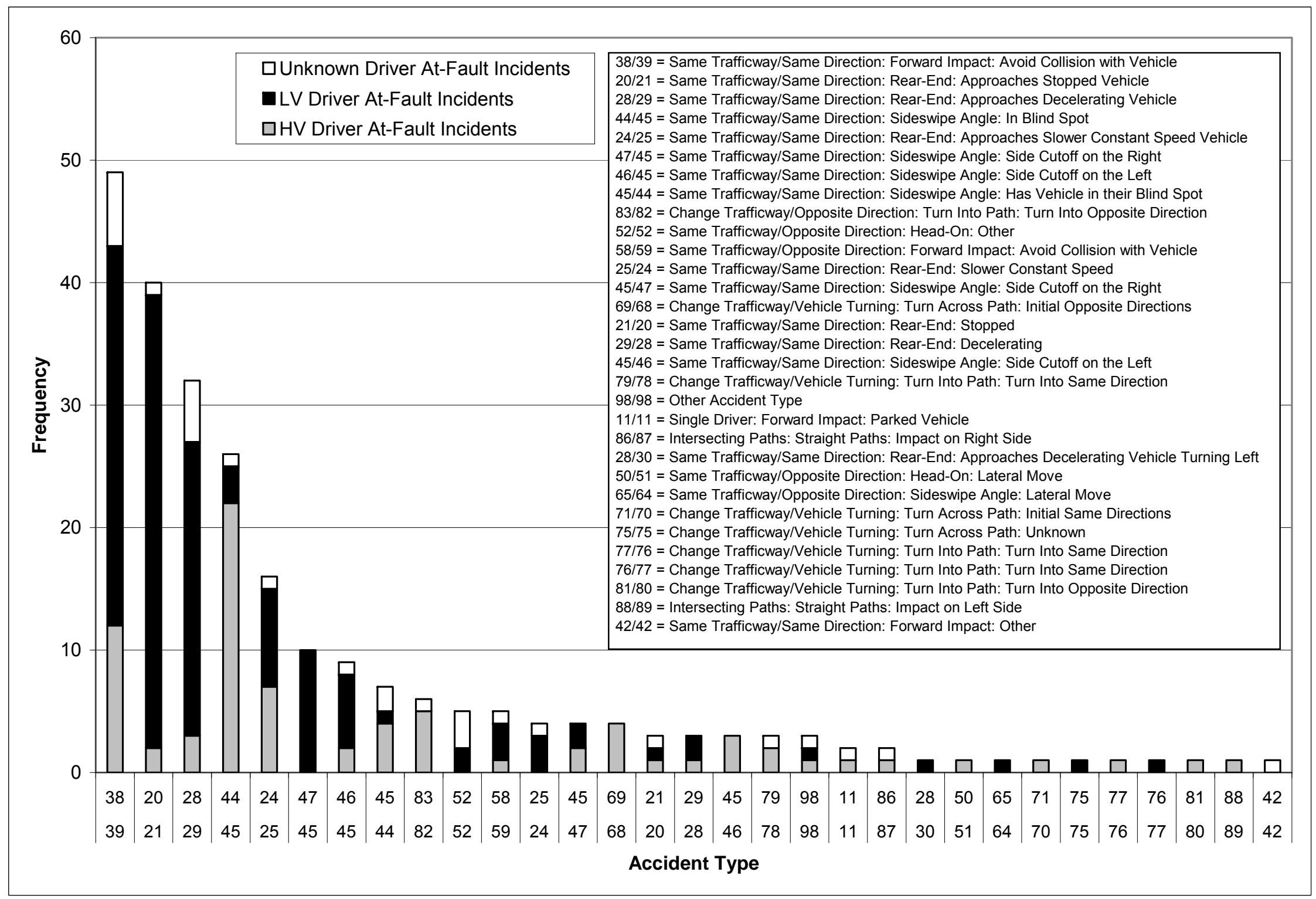

Figure 32. Frequency of Accident Types for HV, LV, and Unknown At-Fault Incidents $\left(\mathbf{n}_{\mathrm{HV}}=79, \mathbf{n}_{\mathrm{LV}}=130, \& \mathbf{n}_{\mathrm{Un}}=29\right)$. 


\section{Accident Types Summary}

Overall, the most frequent Accident Types were Scenarios 38/39: Same Trafficway/Same Direction: Forward Impact: Avoid Collision with Vehicle (19.9\%); 20/21: Same Trafficway/Same Direction: Rear-End: Approaches Stopped Vehicle (16.3\%); and 28/29: Same Trafficway/Same Direction: Rear-End: Approaches Decelerating Vehicle (13\%). These three Accident Types represented 49.2\% of the Accident Types for all LV-HV incidents.

As can be seen in Figure 32, the Accident Types for HV and LV driver at-fault incidents differed markedly. The most prevalent Accident Types for HV driver at-fault incidents were Scenarios 44/45: Same Trafficway/Same Direction: Sideswipe Angle: In Blind Spot (27.8\%); 38/39: Same Trafficway/Same Direction: Forward Impact: Avoid Collision with Vehicle (15.2\%); and 25/25: Same Trafficway/Same Direction: Rear-End: Approaches Slower Constant Speed Vehicle (8.9\%). These three Accident Types accounted for $51.9 \%$ of the HV driver at-fault incidents. The most prevalent Accident Types for LV driver at-fault incidents were Scenarios 20/21: Same Trafficway/Same Direction: Rear-End: Approaches Stopped Vehicle (26.8\%); 38/39: Same Trafficway/Same Direction: Forward Impact: Avoid Collision with Vehicle (22.5\%); and 28/29: Same Trafficway/Same Direction: Rear-End: Approaches Decelerating Vehicle (17.4\%). These three Accident Types accounted for $66.7 \%$ at the LV driver at-fault incidents.

Figure 32 highlights some of the differences between HV and LV driver at-fault incidents with respect to Accident Type. The most prevalent Accident Type for HV driver at-fault incidents involved a Sideswipe Angle. By summing all the HV driver at-fault Accident Types that involved a Sideswipe Angle, it was found that $41.8 \%$ of the HV driver at-fault incidents were coded with this Accident Type. Conversely, the most prevalent Accident Type for LV driver atfault incidents involved a Rear-End Approach. By summing all LV driver at-fault Accident Types that involved a Rear-End approach, it was found that $55.1 \%$ of the LV driver at-fault incidents were coded with this Accident Type. Thus, most of the HV driver at-fault incidents involved a Sideswipe Angle, while most of the LV driver at-fault incident involved a Rear-End Approach.

These results are quite different than Blower's (1998) review of fatal LV-HV crashes. He found that $13.9 \%$ of fatal LV-HV interactions, where only the LV was coded with a driver-related factor, involved a rear-end approach. Further, Blower found that $9.4 \%$ of the fatal LV-HV interactions, where only the $\mathrm{HV}$ driver was coded with a driver-related factor, involved a sideswipe angle. Similarly, when Council et al. (2003) reviewed all types of LV-HV crashes in North Carolina, they found that $23.2 \%$ of the HV driver at-fault crashes involved a sideswipe and $28.5 \%$ of the LV driver at-fault crashes involved a rear-end approach. One possible reason for these discrepancies is due to the difference in the event types being classified; that is, noncrashes in the current study and fatal LV-HV crashes in Blower (1998) and all types of crashes in Council et al. (2003). Also, the driver/subject population that was included in the study was skewed towards high mileage and younger drivers, and this may also impacted the types of events that were recorded in this study. 


\section{Critical Reason for the Critical Event}

To be consistent with the LTCCS (Thieriez, Radja, and Toth, 2002), the LV driver at-fault incidents were coded with a Critical Reason for the incident. The Critical Reason for the incident was considered the primary reason for why the incident occurred (as compared to the Contributing Factors where all the factors that contributed to the incident's occurrence were coded). More than one Critical Reason could be coded for each incident, but this was a rare occurrence (ten of the recorded incidents were coded with two Critical Reasons). Only the LV driver at-fault incidents were coded with a Critical Reason because, as noted previously, only those vehicles were equipped with video recording equipment. For the HV driver at-fault incidents, it was not possible to determine with any certainty what the driver was doing that contributed to the event; therefore, all HV driver at-fault incidents were coded as "Unknown reason for the critical event."

Table 31 shows the frequency, percentage, and rank ordering of the Critical Reasons for LV driver at-fault incidents. The most frequent Critical Reason for the LV driver at-fault incidents was Aggressive Driving Behavior (24.6\%), followed by Too Fast for Conditions (15.2\%) and Internal Distraction (13.8\%). As in the previous analysis to determine the Contributing Factors (Chapter 1), more than one Critical Reason could be coded to a particular incident, thus, the percentage total is greater than 100\%. Figure 33 shows a bar graph of the $138 \mathrm{LV}$ driver at-fault crashes as a function of the Critical Reason. 
Table 31. Frequency, Percentage, and Rank Ordering of the Critical Reasons for LV Driver At-Fault Incidents $\left(\mathbf{n}_{\mathrm{LV}}=\mathbf{1 3 8}\right)$.

\begin{tabular}{|c|c|c|c|}
\hline Critical Reason for the Critical Event & $\begin{array}{l}\text { Frequency of LV } \\
\text { Driver At-Fault } \\
\text { Incidents } \\
\left(n_{\mathrm{LV}}=138\right)\end{array}$ & $\begin{array}{l}\text { Percentage of LV } \\
\text { Driver At-Fault } \\
\text { Incidents } \\
\left(n_{\mathrm{LV}}=138\right)\end{array}$ & $\begin{array}{l}\text { Combined } \\
\text { Rank of LV } \\
\text { Driver At- } \\
\text { Fault } \\
\text { Incidents }\end{array}$ \\
\hline Aggressive driving behavior & 34 & $24.6 \%$ & 1 \\
\hline Too fast for conditions & 21 & $15.2 \%$ & 2 \\
\hline Internal distraction & 19 & $13.8 \%$ & 3 \\
\hline Misjudgment of gap or other's speed & 15 & $10.9 \%$ & 4 \\
\hline $\begin{array}{l}\text { Following too closely to respond to unexpected } \\
\text { actions }\end{array}$ & 10 & $7.2 \%$ & 5.5 \\
\hline False assumption of other road user's actions & 10 & $7.2 \%$ & 5.5 \\
\hline Inadequate surveillance (e.g., failed to look) & 9 & $6.5 \%$ & 7 \\
\hline External distraction & 7 & $5.1 \%$ & 9.5 \\
\hline Inattention & 5 & $3.6 \%$ & 8 \\
\hline Sleep or asleep & 5 & $3.6 \%$ & 9.5 \\
\hline Other recognition error & 3 & $2.2 \%$ & 11 \\
\hline Glare & 2 & $1.4 \%$ & 12.5 \\
\hline Illegal Maneuver & 2 & $1.4 \%$ & 12.5 \\
\hline Blowing debris & 1 & $0.7 \%$ & 16.5 \\
\hline Overcompensation & 1 & $0.7 \%$ & 16.5 \\
\hline Poor directional control & 1 & $0.7 \%$ & 16.5 \\
\hline Other performance error & 1 & $0.7 \%$ & 16.5 \\
\hline Unknown decision error & 1 & $0.7 \%$ & 16.5 \\
\hline Unknown recognition error & 1 & $0.7 \%$ & 16.5 \\
\hline
\end{tabular}




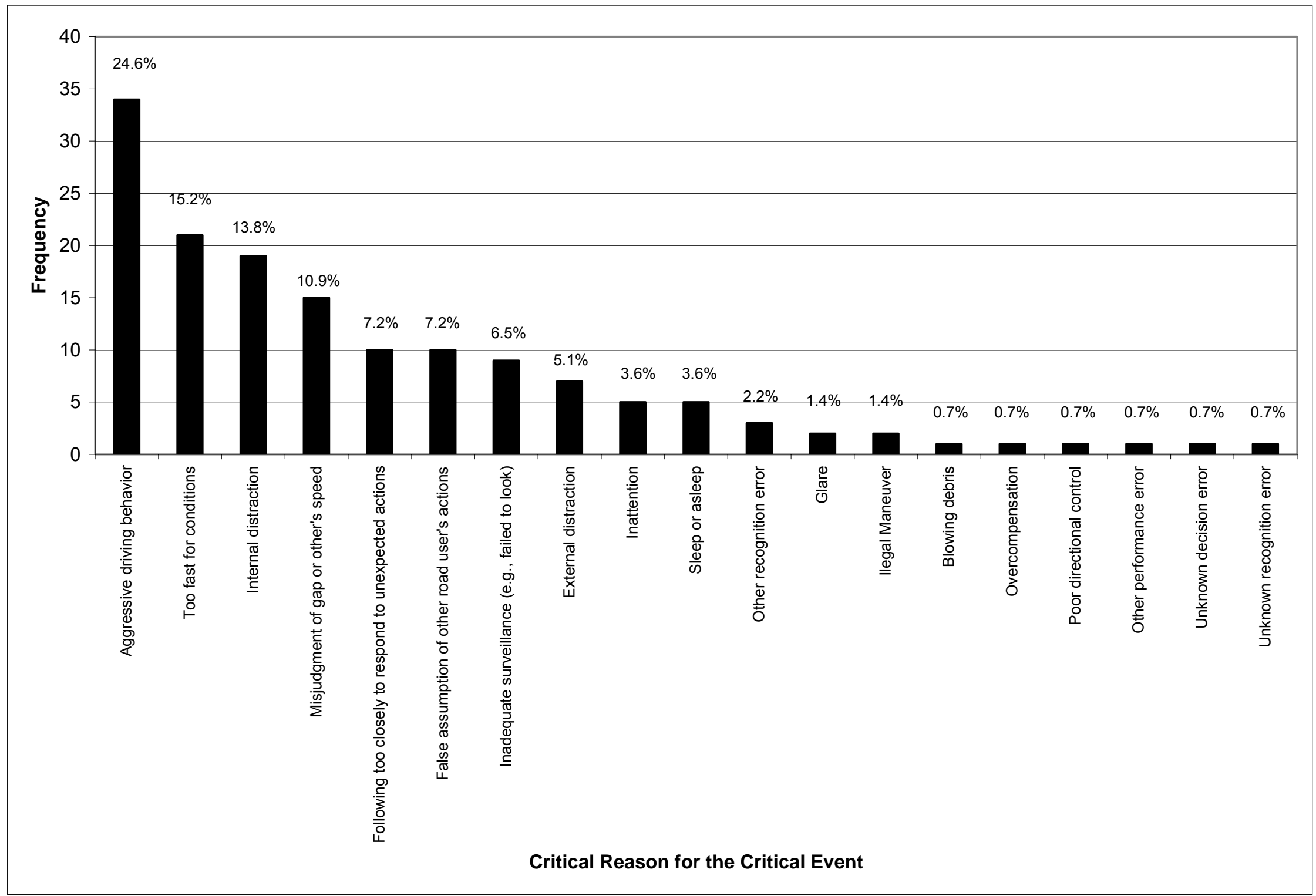

Figure 33. Frequency of Critical Reasons for LV Driver At-Fault Incidents $\left(n_{L V}=138\right)$ 


\section{Critical Reasons X Incident Type Summary}

Table 32 illustrates the frequency of LV driver at-fault incidents by Incident Type as well as Critical Reason. Table 32 provides a more descriptive and comprehensive illustration of the Critical Reasons for each Incident Type. The Incident Types were chosen, rather than the Accident Types, because, based on the results shown previously, they seen to be more appropriate for classifying near-crashes and incidents. The far left column of Table 32 lists the Incident Types while the Critical Reasons are listed in the first row. As indicated above, more than one Critical Reason could be coded to each incident, thus, there are 147 Critical Reasons coded to $138 \mathrm{LV}$ driver at-fault incidents.

As can be seen, the most frequent Critical Reasons for the Late Braking for Stopped/Stopping Traffic Incident Type was Internal Distraction $(n=11)$ and Too Fast for Conditions $(n=8)$. The Internal Distraction and Too Fast For Conditions Critical Reasons were coded in 19.3\% and 14\% LV at-fault Late Braking for Stopped/Stopping Traffic Incident Types, respectively. The most frequent Critical Reasons for the Lane Change Without Sufficient Gap Incident Types were Aggressive Driving $(n=16)$ and Too Fast for Conditions $(n=6)$. The Aggressive Driving and Lane Change Without Sufficient Gap Critical Reasons were coded in 53.3\% and 20\% of the LV at-fault Lane Change Without Sufficient Gap Incident Types, respectively. 
Table 32. Frequency of Incident Types X Critical Reasons for LV Driver At-Fault Incidents $\left(\mathbf{n}_{\mathrm{LV}}=\mathbf{1 3 8 )}\right.$.

\begin{tabular}{|c|c|c|c|c|c|c|c|c|c|c|c|c|c|c|c|c|c|c|c|c|}
\hline 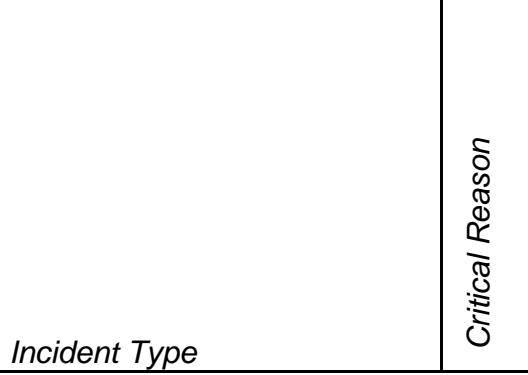 & 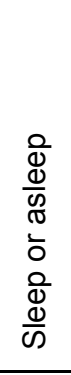 & 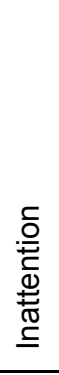 & 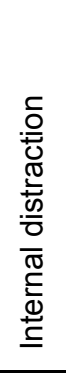 & 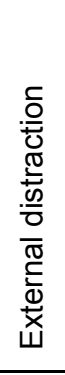 & 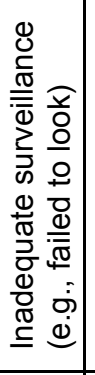 & 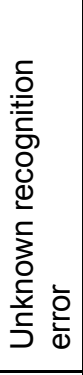 & 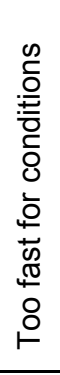 & 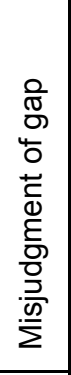 & 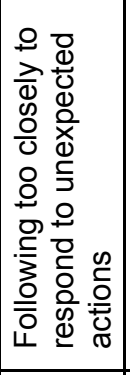 & 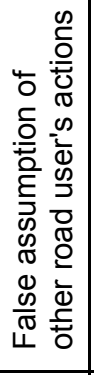 & 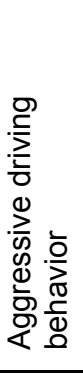 & 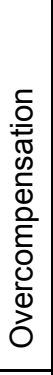 & $\frac{\frac{0}{\sigma}}{0}$ & 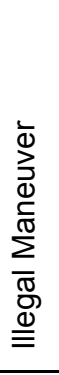 & 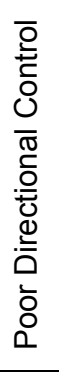 & 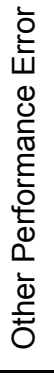 & 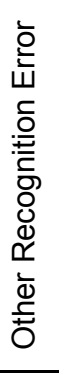 & $\begin{array}{l}\frac{1}{0} \\
\frac{2}{4} \\
\frac{c}{0} \\
\frac{0}{0} \\
\frac{0}{0} \\
0 \\
\frac{5}{3} \\
0 \\
\frac{5}{5} \\
\frac{5}{5}\end{array}$ & $\begin{array}{l}\frac{0}{2} \\
\frac{0}{0} \\
0 \\
0 \\
\frac{5}{3} \\
\frac{0}{0}\end{array}$ & Total \\
\hline Aborted Lane Change & 0 & 0 & 1 & 0 & 3 & 0 & 1 & 3 & 0 & 1 & 2 & 0 & 0 & 0 & 0 & 0 & 0 & 0 & 0 & 11 \\
\hline Approaches Traffic Quickly & 0 & 0 & 1 & 0 & 0 & 0 & 1 & 1 & 0 & 2 & 1 & 0 & 0 & 0 & 0 & 0 & 0 & 0 & 0 & 6 \\
\hline Conflict With Oncoming Traffic & 0 & 1 & 0 & 0 & 0 & 0 & 1 & 0 & 0 & 0 & 2 & 0 & 0 & 0 & 0 & 0 & 0 & 0 & 0 & 4 \\
\hline Following Too Closely & 0 & 1 & 1 & 0 & 0 & 0 & 0 & 0 & 2 & 0 & 0 & 0 & 0 & 0 & 0 & 0 & 0 & 0 & 0 & 4 \\
\hline Improper Lane Change & 0 & 0 & 0 & 0 & 1 & 0 & 0 & 2 & 0 & 0 & 1 & 0 & 0 & 1 & 0 & 0 & 0 & 0 & 0 & 5 \\
\hline Improper Passing & 1 & 0 & 0 & 0 & 0 & 0 & 2 & 0 & 0 & 0 & 6 & 0 & 0 & 0 & 0 & 0 & 0 & 1 & 0 & 10 \\
\hline Improper Stopping at an Intersection & 0 & 0 & 1 & 0 & 0 & 0 & 1 & 0 & 0 & 0 & 0 & 0 & 0 & 0 & 0 & 0 & 0 & 0 & 0 & 2 \\
\hline $\begin{array}{r}\text { Lane Change Without Sufficient } \\
\text { Gap }\end{array}$ & 0 & 0 & 3 & 0 & 2 & 1 & 6 & 2 & 1 & 0 & 16 & 1 & 1 & 1 & 0 & 0 & 0 & 0 & 1 & 35 \\
\hline $\begin{array}{r}\text { Late Braking for Stopped/Stopping } \\
\text { Traffic }\end{array}$ & 2 & 2 & 11 & 7 & 3 & 0 & 8 & 5 & 7 & 6 & 3 & 0 & 1 & 0 & 0 & 1 & 3 & 0 & 0 & 59 \\
\hline Lateral Deviation of Through Vehicle & 0 & 0 & 1 & 0 & 0 & 0 & 0 & 0 & 0 & 0 & 1 & 0 & 0 & 0 & 1 & 0 & 0 & 0 & 0 & 3 \\
\hline Merge Without Sufficient Gap & 2 & 0 & 0 & 0 & 0 & 0 & 1 & 0 & 0 & 1 & 1 & 0 & 0 & 0 & 0 & 0 & 0 & 0 & 0 & 5 \\
\hline $\begin{array}{r}\text { Roadway Entrance Without } \\
\text { Clearance }\end{array}$ & 0 & 0 & 0 & 0 & 0 & 0 & 0 & 2 & 0 & 0 & 0 & 0 & 0 & 0 & 0 & 0 & 0 & 0 & 0 & 2 \\
\hline School Bus Passing Violation & 0 & 1 & 0 & 0 & 0 & 0 & 0 & 0 & 0 & 0 & 1 & 0 & 0 & 0 & 0 & 0 & 0 & 0 & 0 & 2 \\
\hline Total & 5 & 5 & 19 & 7 & 9 & 1 & 21 & 15 & 10 & 10 & 34 & 1 & 2 & 2 & 1 & 1 & 3 & 1 & 1 & 148 \\
\hline
\end{tabular}




\section{Critical Reason Summary}

The most frequent Critical Reasons for LV driver at-fault incidents were Aggressive Driving Behavior (24.6\%), Too Fast for Conditions (15.2\%), and Internal Distraction (13.8\%). These were markedly different than what Blower (1998) found when he assessed fatal LV-HV interactions. For incidents where only the LV driver was cited with a driver-related factor, Blower (1998) found that only 7.1\% of the incidents were coded with the Aggressive Driving Critical Reason, while $19.2 \%$ and $0.11 \%$ of the incidents were coded with Too Fast for Conditions and Internal Distraction, respectively.

There were other interesting trends in the current data set. Sixty-four of the $138 \mathrm{LV}$ at-fault incidents (46.4\%) were coded with at least one Critical Reason that was a risky driving behavior (i.e., Aggressive Driving Behavior, Too Fast for Conditions, Following too Closely, and Illegal Maneuver), while $22.5 \%$ of the LV driver at-fault incidents involved some type of awareness variable (i.e., Internal Distraction, Inattention, External Distraction). In comparison, Stuster's (1999) analysis of fatal LV-HV crashes found that $57.2 \%$ of the incidents involved the at-risk driving behaviors by LV drivers. ${ }^{6}$ Stuster (1999) also found that $10.1 \%$ of fatal LV-HV crashes involved the LV driver driving inattentively.

\section{CONCLUSIONS}

The analyses that were conducted with the LV-HV interactions captured in the 100-Car Study (Dingus et al., 2004) provide convincing evidence to support the contention that LV-HV interactions are a serious problem. Consider that of the 9,125 critical incidents captured in the 100-Car Study, 246 LV-HV interactions were identified. Put another way, of the large critical incident data set that was obtained in the 100-Car Study, $2.7 \%$ of these incidents involved a LV$\mathrm{HV}$ interaction. While $2.7 \%$ may appear to represent a small proportion of the overall critical incident picture, it should be noted that LV-HV interactions easily have the potential to become serious, and even fatal because of the tremendous difference in weight between an $\mathrm{HV}$ and LV.

There are seven important findings that stem from the analyses conducted on the interactions between HVs and LVs. First, of the 246 interactions that were analyzed, 138 (56.1\%) were assessed to have been the fault of the LV driver. HV drivers were at-fault in 79 (32.1\%) of the incidents, while in the remaining 29 (11.8\%) incidents it was unknown if the HV or LV driver was at-fault. Excluding the incidents where it was unknown if the HV or LV driver was at-fault, $63.6 \%$ and $36.4 \%$ of the incidents were the fault of the LV and HV drivers, respectively. Thus, $\mathrm{LV}$ drivers were responsible for a significant proportion of the LV-HV interactions.

These findings support what the drivers in the Hanowski et al. (1998) focus groups reported about LVs being their most important safety concern. Further, the results are similar to prior published studies that used a crash database approach to assess LV-HV interactions (cf. Blower, 1998; Stuster, 1999; Wang, Knipling, and Blincoe, 1999). Based on these findings, it is suggested that focusing on the LV driver, and their errors, may provide the largest area of opportunity for reducing such events.

\footnotetext{
${ }^{6}$ It should be noted that Stuster (1999) only listed the twelve most frequent driver-related factors.
} 
The second important finding from these analyses was in regard to the different Incident Types that were frequent among $\mathrm{HV}$ and $\mathrm{LV}$ drivers. For LV driver at-fault incidents, the most frequent Incident Types were: Late Braking for Stopped/Stopping Traffic (41.3\%), Lane Change Without Sufficient Gap (21.7\%), and Aborted Lane Change (8\%). These particular Incident Types are indicative of at-risk driving behaviors. Once again, the objective data support the sentiment of the L/SH drivers in the Hanowski et al. (1998) focus group who indicated that during their daily travel they were often "cut-off" by LV drivers. And, the data supports the results from the L/SH on-road study (Hanowski, Keisler, and Wierwille, 2004) where the most prevalent Incident Type for LV driver at-fault incident was Lane Change Without Sufficient Gap (accounting for $24.8 \%$ of the $\mathrm{LV}$ driver at-fault incidents in the $\mathrm{L} / \mathrm{SH}$ study). In contrast, the most frequent Incident Types for HV drivers were: Lane Change Without Sufficient Gap (26.6\%), Lateral Deviation of Through Vehicle (21.5\%), and Left Turn Without Clearance (13.9\%). There were substantial differences in the most prevalent Incident Types as a function of driver group.

The third finding is the difference in the Primary Maneuvers for HV and LV drivers. The most prevalent Primary Maneuvers for LV driver at-fault incidents were: Braking (32.6\%), Stopped (21.7\%), and Changing Lanes (16.7\%). The two most frequent Primary Maneuvers for LV driver at-fault incidents involved assumed difficulties on the part of the LV driver decelerating or stopping. In contrast, the most frequent Primary Maneuvers for $\mathrm{HV}$ driver at-fault incidents were: Changing Lanes (32.9\%), Crosses Over Lane Line (20.3\%), and Left Turn (15.2\%). The two most frequent Primary Maneuvers for HV driver at-fault incidents involved difficulties changing or crossing over the lane line while the vehicle was in motion. These results make intuitive sense because HV drivers have limited visibility and deal with blind spots thereby making lane changes difficult in traffic.

It appears that the most frequent Primary Maneuvers for the HV drivers were fundamentally different than those of LV drivers. Whereas the HV drivers seemed to have difficulty changing lanes (which, it is assumed, reflects the inherent difficulty of driving with significant blind spots), LV drivers exhibited difficulties decelerating or stopping (which supports the findings that a significant proportion of the incidents involved a lack of attention and/or at-risk driving behaviors). Also, it is important to point out that there were few similarities in the Primary Maneuvers for LV and HV drivers. This highlights the fundamental differences in the characteristics of at-fault incidents by HV and LV drivers.

The fourth important finding is related to the Contributing Factors that were most frequent with $\mathrm{HV}$ and LV drivers. For LV drivers, the most frequent Contributing Factors for at-fault incidents were: Driving Techniques (70.3\%), Distracted (22.5\%), and Aggressive Driving (22.5\%). The most frequent Contributing Factors for HV driver at-fault incidents were: Unknown (68.4\%), Driving Techniques (15.2\%), and Distracted (11.4\%). The large number of Unknown Contributing Factors for HV driver at-fault incidents is indicative of the methodology used to code these events. Because the HV did not have any video cameras, the Contributing Factor was coded with respect to the behaviors of the LV driver. As the LV driver was not responsible for the incident, it was unlikely they would be coded with a Contributing Factor, thus the high frequency of Unknown Contributing Factors. Further, the methodology used to code the 
Contributing Factors also explains the similarities between LVs and HVs (i.e., they were all coded with respect to the LV driver, and therefore, might be expected to be similar).

The fifth noteworthy finding from the current research involves the Accident Types that were most prevalent for LV and HV drivers. The most prevalent Accident Types for LV driver atfault incidents were: Scenarios 20/21: Same Trafficway/Same Direction: Rear End: Approaches Stopped Vehicle (26.8\%); 38/39: Same Trafficway/Same Direction: Forward Impact: Avoid Collision with Vehicle (22.5\%); and 28/29: Same Trafficway/Same Direction: Rear End: Approaches Decelerating Vehicle (17.4\%). Approximately 55\% of the LV driver at-fault incidents involved a Rear-End approach. These Accident Types also support the findings from the analysis of the most prevalent Primary Maneuvers for LV driver at-fault incidents: decelerating or stopped.

Conversely, the most prevalent Accident Types for HV driver at-fault incidents were: Scenarios 44/45: Same Trafficway/Same Direction: Forward Impact: Sideswipe Angle: In Blind Spot (27.7\%); 38/39: Same Trafficway/Same Direction: Forward Impact: Avoid Collision with Vehicle (15.2\%); and 25/25: Same Trafficway/Same Direction: Rear End: Approaches Constant Speed Vehicle (8.9\%). Approximately $42 \%$ of the HV driver at-fault incidents involved a Sideswipe Angle. These Accident Types also support the findings from the most prevalent Primary Maneuvers for HV driver at-fault incidents: changing lanes and crossing the lane line.

The seventh noteworthy finding from the current research reflects some of the similarities and discrepancies found between the current study and prior studies using a crash database approach in analyzing $\mathrm{LV}-\mathrm{HV}$ interactions. While both approaches found that $\mathrm{LV}$ drivers were responsible for the majority of $\mathrm{LV}-\mathrm{HV}$ interactions, the reasons why these interactions occurred differed with respect to the methodologies used to assess these interactions. For example, the current research found that $22.5 \%$ of the LV driver at-fault incidents were cited with the Contributing Factors of Aggressive Driving. In Stuster's (1999) analysis, only 4.3\% of the LVs were cited with the driver-related factor "Erratic/Reckless Driving." Moreover, Hankey et al. (1999) found that $31.1 \%$ of the fatal crashes in the FARS database were cited with Aggressive Driving. As such, the results from the current study (22.5\%) are within the range reported by Stuster (4.3\%) and Hankey (31.1\%).

Table 33 compares the Critical Reasons for the LV at-fault incidents in the current study to the LV driver-related factors in the Blower (1998), Stuster (1999), and Council et al. (2003) studies. Although Table 33 provides a convenient way to compare the prior LV-HV interaction studies to the current study, the reader should be aware that there were significant differences in the data and classification strategy used in the different studies. For example, both Blower (1998) and Stuster (1999) assessed only fatal LV-HV crashes, and Council et al. (2003) assessed all types of LV-HV crashes (including fatal crashes). More than one driver-related factor could have been selected in the Blower (1998), Stuster (1999), and Council et al (2003) studies. Lastly, the Stuster (1999) study only reported the twelve most important (or frequent) driver-related factors. 
Table 33. Comparison of a Selection of Results in the Current Study with Prior LV-HV Interaction Studies.

\begin{tabular}{|l|c|c|c|c|c|}
\hline \multicolumn{1}{|c|}{ Condition } & $\begin{array}{c}\text { 100-Car Study } \\
\text { (Current Study) }\end{array}$ & $\begin{array}{c}\text { Hanowski } \\
\text { et al. } \\
\text { (2004) }\end{array}$ & $\begin{array}{c}\text { Blower } \\
\text { (1998) }\end{array}$ & Stuster (1999) & Council et al. (2003) \\
\hline LV At-Fault & $56.1 \%$ & $78.1 \%$ & $70.3 \%$ & $29 \%$ & $40.2 \%$ \\
\hline HV At-Fault & $32.1 \%$ & 21.9 & $16.2 \%$ & $67 \%$ & $48 \%$ \\
\hline Distraction Incidents & $22.4 \%$ & $6.5 \%^{7}$ & $11.2 \%$ & $8.7 \%^{8}$ & $\mathrm{~N} / \mathrm{A}$ \\
\hline $\begin{array}{l}\text { Too Fast for Conditions } \\
\text { Incidents }\end{array}$ & $15.2 \%$ & $\mathrm{~N} / \mathrm{A}$ & $20.3 \%$ & $14.1 \%{ }^{9}$ & $5.2 \% / 14.5 \% \%^{9,10}$ \\
\hline & & & & & \\
$\begin{array}{l}\text { Following Too Closely } \\
\text { Incidents }\end{array}$ & $7.2 \%$ & $2.9 \%$ & $3.4 \%^{11}$ & $2.7 \%$ & $\mathrm{~N} / \mathrm{A}$ \\
\hline Aggressive Driving Incidents & $24.6 \%$ & $37 \%$ & $7.1 \%^{12}$ & $4.3 \%^{12}$ & $\mathrm{~N} / \mathrm{A}$ \\
\hline
\end{tabular}

The current research also found that $41.8 \%$ of the $\mathrm{HV}$ driver at-fault incidents involved a Sideswipe Angle, while $55.1 \%$ of the LV driver at-fault incidents involved a Rear End approach. These results differed from Blower's (1998) review of fatal LV-HV crashes. He found that 9.4\% of fatal LV-HV interactions involved a sideswipe angle. Further, Blower's (1998) analysis found that $13.9 \%$ of the fatal LV-HV interactions involved a rear-end strike. When Council et al. (2003) reviewed all types of LV-HV crashes in North Carolina, they found that $23.2 \%$ of the HV driver at-fault crashes involved a sideswipe and $28.5 \%$ of the LV driver at-fault crashes involved a rear-end approach. These discrepancies might highlight the differences between analyzing crashes and near crashes and/or the methodologies used analyze the data (i.e., a crash database approach versus a naturalistic or in situ data collection approach, and the younger age bias in the current study).

\footnotetext{
${ }^{7}$ Only includes HV driver initiated events recorded in the Sleeper Berth study (Hanowski, Olson, Perez, and Dingus, 2001).

${ }^{8}$ Only includes driving inattentively.

${ }^{9}$ Called “Unsafe Speed” rather than Too Fast for Conditions in Stuster (1999) and Council et al. (2003).

${ }^{10}$ The first number indicates the percent of all LV-HV crashes, while the second number indicates the percent of fatal LV-HV crashes

${ }^{11}$ Called “Improper Following” rather than Following Too Closely in Blower (1998).

12 Called "Erratic/Reckless Driving” in both Blower (1998) and Stuster (1999).
} 


\section{CHAPTER 2: COMPARISON OF THE RESULTS FROM THE ANALYSES CONDUCTED WITH THE 100-CAR DATA AND THE LOCAL/SHORT HAUL AND SLEEPER BERTH DATA}

Recall in Chapter 1 that analyses were conducted with critical incident data that were collected during the 100-Car Study (Dingus et al., 2004). The method of analysis used in Chapter 1 is almost identical to the approach used in Hanowski, Keisler, and Wierwille (2004). The Hanowski, Keisler, and Wierwille (2004) study assessed LV-HV interactions from the perspective of local/short-haul (L/SH) and sleeper berth (SB) drivers. A total of $142 \mathrm{LV}-\mathrm{HV}$ interactions were identified in the L/SH study (see Hanowski, Wierwille, Garness, and Dingus, 2000, for a complete description of the L/SH study). Of these, 117 (82.4\%) incidents were judged to have been the fault of the LV driver, while the remaining 25 (17.6\%) incidents were the fault of the HV driver (remember that incidents in the L/SH and SB studies used "initiate" to indicate fault. From this point on, "at-fault” will be used rather than "initiate”).

In the SB study, a total of $68 \mathrm{LV}-\mathrm{HV}$ interactions were identified (see Dingus et al., 2002, for a complete description of the SB study). Of these, 47 (69.1\%) were assessed to have been the fault of the LV driver, while the remaining 21 (38.9\%) were the fault of the HV driver. Taken together with the current research, these three studies consistently show that LV drivers appear to be responsible for the majority of LV-HV interactions. Of the $427 \mathrm{LV}-\mathrm{HV}$ incidents identified across the three studies (excluding the 29 Unknown at-fault incidents in the current study), 302 (70.7\%) were the fault of the LV driver, while the remaining 125 (29.3\%) were the fault of the HV driver (a 2.4:1 ratio).

However, one of the weaknesses in the current study was the lack of instrumentation in HVs. Conversely, one of the weaknesses in the Hanowski, Keisler, and Wierwille (2004) study was the lack of instrumentation in LVs. Thus, by integrating the results from the present study with the results from the Hanowski, Keisler, and Wierwille (2004) study, it is believed that a more complete understanding of LV-HV interaction problem can be gained. Chapter 2 has two primary aims: combine the data from the current study with the data from the L/SH and SB studies in Hanowski, Keisler, and Wierwille (2004) to illustrate the overall LV-HV interaction picture, and address the limitation of having only one vehicle instrumented by assessing the differences between the three studies (i.e., are the recorded LV-HV interactions fundamentally different as a function of which vehicle is instrumented?).

\section{INCIDENT TYPES}

Table 34 illustrates the frequency, percentage, and rank ordering of Incident Types across all three studies. As can be seen in Table 34, there were a total of $456 \mathrm{LV}-\mathrm{HV}$ interactions across the three studies. The most frequent Incident Type was Lane Change Without Sufficient Gap (22.1\%), followed by Late Braking for Stopped/Stopping Traffic (17.3\%) and Roadway Entrance Without Clearance (7.9\%). Figure 34 shows a bar graph of the 456 incidents, across the three studies, as a function of Incident Type. 
Table 34. Frequency, Percentage, and Rank Ordering of the Incident Types Across the Three Studies.

\begin{tabular}{|c|c|c|c|}
\hline Incident Type & $\begin{array}{l}\text { Frequency of } \\
\text { Incidents } \\
\text { Across all } \\
\text { Three Studies }\end{array}$ & $\begin{array}{l}\text { Percentage of } \\
\text { Incidents } \\
\text { Across all } \\
\text { Three Studies }\end{array}$ & $\begin{array}{c}\text { Combined } \\
\text { Rank }\end{array}$ \\
\hline Lane Change Without Sufficient Gap & 101 & $22.1 \%$ & 1 \\
\hline Late Braking for Stopped/Stopping Traffic & 79 & $17.3 \%$ & 2 \\
\hline Roadway Entrance Without Clearance & 36 & $7.9 \%$ & 3 \\
\hline Left Turn Without Clearance & 34 & $7.5 \%$ & 4 \\
\hline Lateral Deviation of Through Vehicle & 25 & $5.5 \%$ & 5 \\
\hline Improper Passing & 21 & $4.6 \%$ & 6 \\
\hline Slow Speed & 16 & $3.5 \%$ & 7 \\
\hline Aborted Lane Change & 15 & $3.3 \%$ & 8.5 \\
\hline Turn Without Sufficient Warning & 15 & $3.3 \%$ & 8.5 \\
\hline Obstruction in Roadway & 13 & $2.9 \%$ & 10 \\
\hline Following Too Closely & 11 & $2.4 \%$ & 11 \\
\hline Wide Turn Into Adjacent Lane & 10 & $2.2 \%$ & 12 \\
\hline Merge Without Sufficient Gap & 9 & $2.0 \%$ & 13 \\
\hline Backing in Roadway & 8 & $1.8 \%$ & 15 \\
\hline Conflict With Oncoming Traffic & 8 & $1.8 \%$ & 15 \\
\hline Through Traffic Does Not Allow Merge & 8 & $1.8 \%$ & 15 \\
\hline Approaches Traffic Quickly & 6 & $1.3 \%$ & 17 \\
\hline Turn/Exit From Incorrect Lane & 5 & $1.1 \%$ & 19 \\
\hline Merge Out Of Turn (Before Lead Vehicle) & 5 & $1.1 \%$ & 19 \\
\hline Slow Upon Passing & 5 & $1.1 \%$ & 19 \\
\hline Improper Lane Change & 4 & $0.9 \%$ & 21.5 \\
\hline Exit Then Re-Entrance onto Roadway & 4 & $0.9 \%$ & 21.5 \\
\hline Unable to Determine & 3 & $0.7 \%$ & 23 \\
\hline Clear Path for Emergency Vehicle & 2 & $0.4 \%$ & 26 \\
\hline Improper Stopping at an Intersection & 2 & $0.4 \%$ & 26 \\
\hline School Bus Passing Violation & 2 & $0.4 \%$ & 26 \\
\hline Through Traffic Does Not Allow Lane Change & 2 & $0.4 \%$ & 26 \\
\hline Conflict Between Merging and Exiting Traffic & 2 & $0.4 \%$ & 26 \\
\hline Improper U-Turn & 1 & $0.2 \%$ & 31 \\
\hline Improperly Covered Debris from Lead Vehicle & 1 & $0.2 \%$ & 31 \\
\hline Sudden Braking in Roadway & 1 & $0.2 \%$ & 31 \\
\hline Obscene Gesture (To Other Driver) & 1 & $0.2 \%$ & 31 \\
\hline Proceeding Through Red Traffic Signal & 1 & $0.2 \%$ & 31 \\
\hline
\end{tabular}




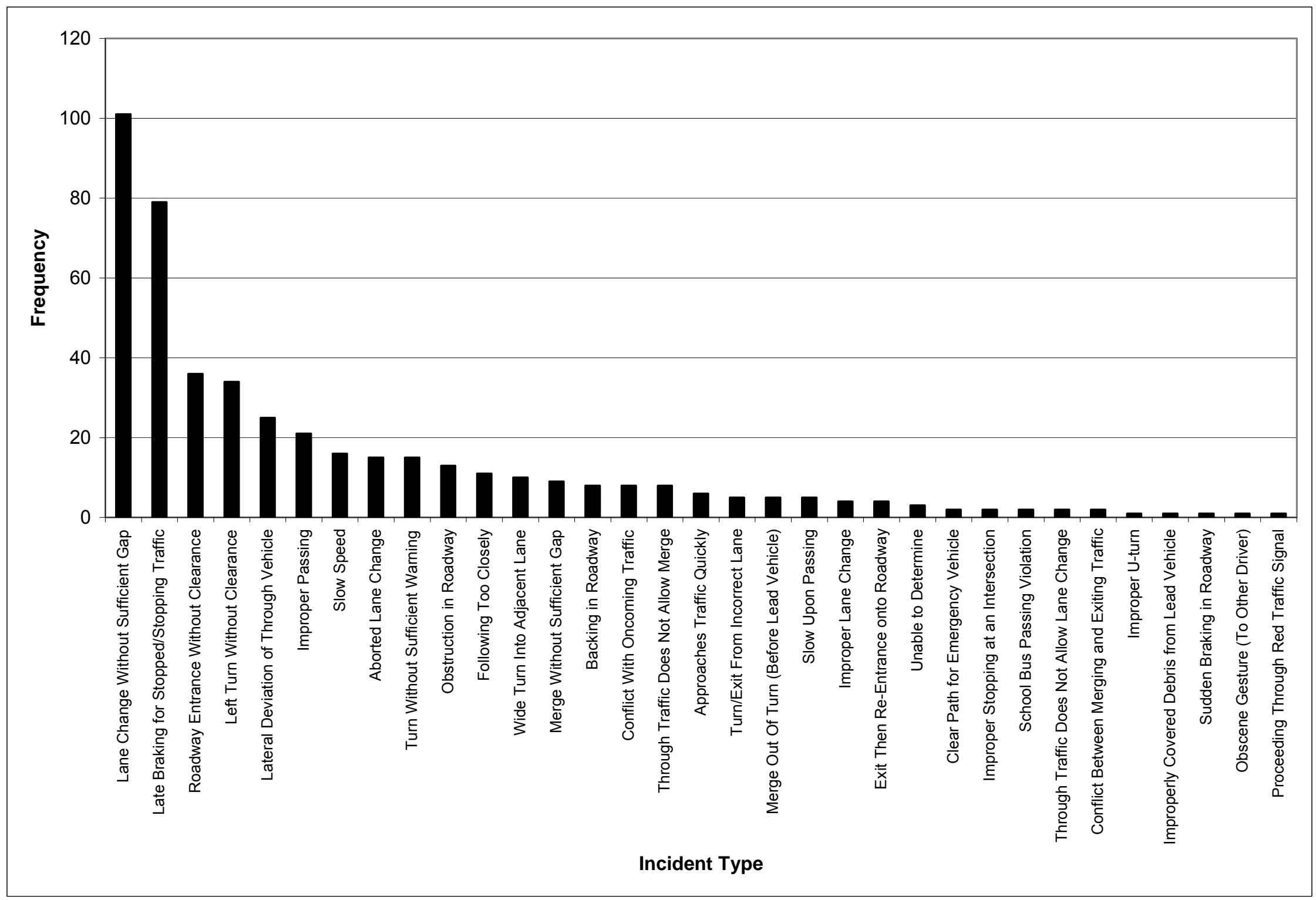

Figure 34. Percentage of Incident Types Across all Three Studies. 
Tables 35-37 show the frequency and percentage of each Incident Type in the 100-Car, SB, and L/SH studies, respectively. In the 100-Car Study, the most frequent Incident Types were Late Braking for Stopped/Stopping Traffic (26.8\%), Lane Change Without Sufficient Gap (22\%), and Lateral Deviation of Through Vehicle (8.1\%). In the SB study, the most frequent Incident Types were Lane Change Without Sufficient Gap (25\%), Turn Without Sufficient Warning (17.6\%), Late Braking for Stopped/Stopping Traffic (14.7\%). In the L/SH study, the most frequent Incident Types were Lane Change Without Sufficient Gap (21.1\%), Roadway Entrance Without Clearance (14.3\%), and Left Turn Without Clearance (14.8\%). Figure 35 shows a bar graph that illustrates the percentage of incidents in the 100-Car, SB, and L/SH studies as a function of the Incident Types. 
Table 35. Frequency and Percentage of Incident Types for the 100-Car Study.

\begin{tabular}{|c|c|c|c|c|c|c|c|c|}
\hline Incident Type & $\begin{array}{c}\text { Frequency of } \\
\text { 100-Car Driver } \\
\text { At-Fault } \\
\text { Incidents } \\
\left(\mathrm{n}_{100-\text {-Car }}=138\right)\end{array}$ & $\begin{array}{c}\text { Percentage of } \\
\text { 100-Car Driver } \\
\text { At-Fault } \\
\text { Incidents } \\
\left(\mathrm{n}_{100-\mathrm{Car}}=138\right)\end{array}$ & $\begin{array}{l}\text { Frequency of } \\
\text { HV Driver At- } \\
\text { Fault } \\
\text { Incidents } \\
\left(n_{\mathrm{HV}}=79\right)\end{array}$ & $\begin{array}{l}\text { Percentage of } \\
\text { HV Driver At- } \\
\text { Fault } \\
\text { Incidents } \\
\left(\mathbf{n}_{\mathrm{HV}}=79\right)\end{array}$ & $\begin{array}{l}\text { Frequency of } \\
\text { Unknown } \\
\text { Driver At-Fault } \\
\text { Incidents } \\
\left(n_{\text {Un }}=29\right)\end{array}$ & $\begin{array}{l}\text { Percentage of } \\
\text { Unknown } \\
\text { Driver At-Fault } \\
\text { Incidents } \\
\left(n_{\text {Un }}=29\right)\end{array}$ & $\begin{array}{l}\text { Frequency of } \\
\text { All Incidents } \\
\left(\mathrm{N}_{\text {Total }}=246\right)\end{array}$ & $\begin{array}{l}\text { Percentage of } \\
\text { All Incidents } \\
\left(N_{\text {Total }}=246\right)\end{array}$ \\
\hline $\begin{array}{l}\text { Late Braking for } \\
\text { Stopped/Stopping Traffic }\end{array}$ & 57 & $41.3 \%$ & 1 & $1.3 \%$ & 8 & $27.6 \%$ & 66 & $26.8 \%$ \\
\hline $\begin{array}{l}\text { Lane Change Without Sufficient } \\
\text { Gap }\end{array}$ & 30 & $21.7 \%$ & 21 & $26.6 \%$ & 3 & $10.3 \%$ & 54 & $22.0 \%$ \\
\hline $\begin{array}{l}\text { Lateral Deviation of Through } \\
\text { Vehicle }\end{array}$ & 3 & $2.2 \%$ & 17 & $21.5 \%$ & 0 & $0.0 \%$ & 20 & $8.1 \%$ \\
\hline Aborted Lane Change & 11 & $8.0 \%$ & 4 & $5.1 \%$ & 0 & $0.0 \%$ & 15 & $6.1 \%$ \\
\hline Left Turn Without Clearance & 0 & $0.0 \%$ & 11 & $13.9 \%$ & 2 & $6.9 \%$ & 13 & $5.3 \%$ \\
\hline Improper Passing & 10 & $7.2 \%$ & 0 & $0.0 \%$ & 2 & $6.9 \%$ & 12 & $4.9 \%$ \\
\hline Merge Without Sufficient Gap & 5 & $3.6 \%$ & 3 & $3.8 \%$ & 1 & $3.4 \%$ & 9 & $3.7 \%$ \\
\hline Approaches Traffic Quickly & 6 & $4.3 \%$ & 0 & $0.0 \%$ & 0 & $0.0 \%$ & 6 & $2.4 \%$ \\
\hline $\begin{array}{l}\text { Roadway Entrance Without } \\
\text { Clearance }\end{array}$ & 2 & $1.4 \%$ & 2 & $2.5 \%$ & 2 & $6.9 \%$ & 6 & $2.4 \%$ \\
\hline Following Too Closely & 4 & $2.9 \%$ & 1 & $1.3 \%$ & 0 & $0.0 \%$ & 5 & $2.0 \%$ \\
\hline Obstruction in Roadway & 0 & $0.0 \%$ & 4 & $5.1 \%$ & 1 & $3.4 \%$ & 5 & $2.0 \%$ \\
\hline Improper Lane Change & 3 & $2.2 \%$ & 1 & $1.3 \%$ & 0 & $0.0 \%$ & 4 & $1.6 \%$ \\
\hline $\begin{array}{l}\text { Through Traffic Does Not Allow } \\
\text { Merge }\end{array}$ & 0 & $0.0 \%$ & 3 & $3.8 \%$ & 0 & $0.0 \%$ & 3 & $1.2 \%$ \\
\hline Unable to Determine & 0 & $0.0 \%$ & 0 & $0.0 \%$ & 3 & $10.3 \%$ & 3 & $1.2 \%$ \\
\hline Clear Path for Emergency Vehicle & 0 & $0.0 \%$ & 1 & $1.3 \%$ & 1 & $3.4 \%$ & 2 & $0.8 \%$ \\
\hline $\begin{array}{l}\text { Improper Stopping at an } \\
\text { Intersection }\end{array}$ & 2 & $1.4 \%$ & 0 & $0.0 \%$ & 0 & $0.0 \%$ & 2 & $0.8 \%$ \\
\hline School Bus Passing Violation & 2 & $1.4 \%$ & 0 & $0.0 \%$ & 0 & $0.0 \%$ & 2 & $0.8 \%$ \\
\hline
\end{tabular}




\begin{tabular}{|c|c|c|c|c|c|c|c|c|}
\hline Incident Type & $\begin{array}{c}\text { Frequency of } \\
\text { 100-Car Driver } \\
\text { At-Fault } \\
\text { Incidents } \\
\left(\mathrm{n}_{100-C a}=138\right)\end{array}$ & $\begin{array}{l}\text { Percentage of } \\
\text { 100-Car Driver } \\
\text { At-Fault } \\
\text { Incidents } \\
\left(n_{100-C a r}=138\right)\end{array}$ & $\begin{array}{c}\text { Frequency of } \\
\text { HV Driver At- } \\
\text { Fault } \\
\text { Incidents } \\
\left(\mathrm{n}_{\mathrm{HV}}=79\right)\end{array}$ & $\begin{array}{l}\text { Percentage of } \\
\text { HV Driver At- } \\
\text { Fault } \\
\text { Incidents } \\
\left(\mathrm{n}_{\mathrm{HV}}=79\right)\end{array}$ & $\begin{array}{l}\text { Frequency of } \\
\text { Unknown } \\
\text { Driver At-Fault } \\
\text { Incidents } \\
\text { (nun }=29)\end{array}$ & $\begin{array}{l}\text { Percentage of } \\
\text { Unknown } \\
\text { Driver At-Fault } \\
\text { Incidents } \\
\left(n_{U n}=29\right)\end{array}$ & $\begin{array}{l}\text { Frequency of } \\
\text { All Incidents } \\
\left(N_{\text {Total }}=246\right)\end{array}$ & $\begin{array}{l}\text { Percentage of } \\
\text { All Incidents } \\
\left(\mathrm{N}_{\text {Total }}=246\right)\end{array}$ \\
\hline $\begin{array}{l}\text { Through Traffic Does Not Allow } \\
\text { Lane Change }\end{array}$ & 0 & $0.0 \%$ & 0 & $0.0 \%$ & 2 & $6.9 \%$ & 2 & $0.8 \%$ \\
\hline Wide Turn Into Adjacent Lane & 0 & $0.0 \%$ & 2 & $2.5 \%$ & 0 & $0.0 \%$ & 2 & $0.8 \%$ \\
\hline Improper U-Turn & 0 & $0.0 \%$ & 1 & $1.3 \%$ & 0 & $0.0 \%$ & 1 & $0.4 \%$ \\
\hline $\begin{array}{l}\text { Improperly Covered Debris from } \\
\text { Lead Vehicle }\end{array}$ & 0 & $0.0 \%$ & 1 & $1.3 \%$ & 0 & $0.0 \%$ & 1 & $0.4 \%$ \\
\hline Slow Speed & 0 & $0.0 \%$ & 1 & $1.3 \%$ & 0 & $0.0 \%$ & 1 & $0.4 \%$ \\
\hline Sudden Braking in Roadway & 0 & $0.0 \%$ & 1 & $1.3 \%$ & 0 & $0.0 \%$ & 1 & $0.4 \%$ \\
\hline Turn Without Sufficient Warning & 0 & $0.0 \%$ & 1 & $1.3 \%$ & 0 & $0.0 \%$ & 1 & $0.4 \%$ \\
\hline
\end{tabular}


Table 36. Frequency and Percentage of Incident Types for the Sleeper Berth Study.

\begin{tabular}{|c|c|c|c|c|c|c|}
\hline Incident Type & $\begin{array}{c}\text { Frequency of } \\
\text { SB Driver At- } \\
\text { Fault } \\
\text { Incidents } \\
\left(n_{\mathrm{SB}}=21\right)\end{array}$ & $\begin{array}{l}\text { Percentage of } \\
\text { SB Driver At- } \\
\text { Fault Incidents } \\
\quad\left(n_{\mathrm{SB}}=21\right)\end{array}$ & $\begin{array}{l}\text { Frequency of } \\
\text { LV Driver At- } \\
\text { Fault Incidents } \\
\left(n_{\mathrm{LV}}=47\right)\end{array}$ & $\begin{array}{l}\text { Percentage of } \\
\text { LV Driver At- } \\
\text { Fault Incidents } \\
\quad\left(n_{\mathrm{LV}}=47\right)\end{array}$ & $\begin{array}{l}\text { Frequency of } \\
\text { All Incidents } \\
\left(\mathrm{N}_{\text {Total }}=68\right)\end{array}$ & $\begin{array}{c}\text { Percentage of } \\
\text { All Incidents } \\
\left(\mathrm{N}_{\text {Total }}=68\right)\end{array}$ \\
\hline Lane Change Without Sufficient Gap & 2 & $9.5 \%$ & 15 & $31.9 \%$ & 17 & $25.0 \%$ \\
\hline Turn Without Sufficient Warning & 3 & $14.3 \%$ & 9 & $19.1 \%$ & 12 & $17.6 \%$ \\
\hline $\begin{array}{l}\text { Late Braking For Stopped/ Stopping } \\
\text { Traffic }\end{array}$ & 10 & $47.6 \%$ & 0 & $0.0 \%$ & 10 & $14.7 \%$ \\
\hline Low Speed & 1 & $4.8 \%$ & 8 & $17.0 \%$ & 9 & $13.2 \%$ \\
\hline Following Too Closely & 5 & $23.8 \%$ & 0 & $0.0 \%$ & 5 & $7.4 \%$ \\
\hline Roadway Entrance Without Clearance & 0 & $0.0 \%$ & 4 & $8.5 \%$ & 4 & $5.9 \%$ \\
\hline Obstruction In Roadway & 0 & $0.0 \%$ & 4 & $8.5 \%$ & 4 & $5.9 \%$ \\
\hline Lateral Deviation Of Through Vehicle & 0 & $0.0 \%$ & 3 & $6.4 \%$ & 3 & $4.4 \%$ \\
\hline Improper Passing & 0 & $0.0 \%$ & 2 & $4.3 \%$ & 2 & $2.9 \%$ \\
\hline Slow Upon Passing & 0 & $0.0 \%$ & 2 & $4.3 \%$ & 2 & $2.9 \%$ \\
\hline Left Turn Without Clearance & 0 & $0.0 \%$ & 0 & $0.0 \%$ & 0 & $0.0 \%$ \\
\hline Wide Turn Into Adjacent Lane & 0 & $0.0 \%$ & 0 & $0.0 \%$ & 0 & $0.0 \%$ \\
\hline Backing In Roadway & 0 & $0.0 \%$ & 0 & $0.0 \%$ & 0 & $0.0 \%$ \\
\hline Merge Out Of Turn & 0 & $0.0 \%$ & 0 & $0.0 \%$ & 0 & $0.0 \%$ \\
\hline Through Traffic Does Not Allow Merge & 0 & $0.0 \%$ & 0 & $0.0 \%$ & 0 & $0.0 \%$ \\
\hline Exit Then Re-Entrance onto Roadway & 0 & $0.0 \%$ & 0 & $0.0 \%$ & 0 & $0.0 \%$ \\
\hline Turn/ Exit From Incorrect Lane & 0 & $0.0 \%$ & 0 & $0.0 \%$ & 0 & $0.0 \%$ \\
\hline $\begin{array}{l}\text { Conflict Between Merging and Exiting } \\
\text { Traffic }\end{array}$ & 0 & $0.0 \%$ & 0 & $0.0 \%$ & 0 & $0.0 \%$ \\
\hline Obscene Gesture (To Other Driver) & 0 & $0.0 \%$ & 0 & $0.0 \%$ & 0 & $0.0 \%$ \\
\hline Proceeding Through Red Traffic Signal & 0 & $0.0 \%$ & 0 & $0.0 \%$ & 0 & $0.0 \%$ \\
\hline
\end{tabular}


Table 37. Frequency and Percentage of Incident Types for the Local/Short Haul Study.

\begin{tabular}{|c|c|c|c|c|c|c|}
\hline Incident Type & $\begin{array}{l}\text { Frequency of } \\
\text { L/SH Driver At- } \\
\text { Fault Incidents } \\
\left(n_{\mathrm{L} / \mathrm{SH}}=25\right)\end{array}$ & $\begin{array}{l}\text { Percentage of } \\
\text { L/SH Driver At- } \\
\text { Fault Incidents } \\
\left(n_{\mathrm{L} / S H}=25\right)\end{array}$ & $\begin{array}{l}\text { Frequency of } \\
\text { LV Driver At- } \\
\text { Fault Incidents } \\
\left(n_{\mathrm{LV}}=117\right)\end{array}$ & $\begin{array}{l}\text { Percentage of } \\
\text { LV Driver At- } \\
\text { Fault Incidents } \\
\left(n_{\mathrm{LV}}=117\right)\end{array}$ & $\begin{array}{l}\text { Frequency of } \\
\text { All Incidents } \\
\left(N_{\text {Total }}=142\right)\end{array}$ & $\begin{array}{l}\text { Percentage of } \\
\text { All Incidents } \\
\left(\mathrm{N}_{\text {Total }}=142\right)\end{array}$ \\
\hline Lane Change Without Sufficient Gap & 1 & $4.0 \%$ & 29 & $24.8 \%$ & 30 & $21.1 \%$ \\
\hline Roadway Entrance Without Clearance & 5 & $20.0 \%$ & 21 & $17.9 \%$ & 26 & $18.3 \%$ \\
\hline Left Turn Without Clearance & 0 & $0.0 \%$ & 21 & $17.9 \%$ & 21 & $14.8 \%$ \\
\hline Wide Turn Into Adjacent Lane & 3 & $12.0 \%$ & 5 & $4.3 \%$ & 8 & $5.6 \%$ \\
\hline Improper Passing & 1 & $4.0 \%$ & 6 & $5.1 \%$ & 7 & $4.9 \%$ \\
\hline Backing In Roadway & 3 & $12.0 \%$ & 4 & $3.4 \%$ & 7 & $4.9 \%$ \\
\hline Low Speed & 2 & $8.0 \%$ & 4 & $3.4 \%$ & 6 & $4.2 \%$ \\
\hline Merge Out Of Turn & 1 & $4.0 \%$ & 4 & $3.4 \%$ & 5 & $3.5 \%$ \\
\hline Through Traffic Does Not Allow Merge & 0 & $0.0 \%$ & 5 & $4.3 \%$ & 5 & $3.5 \%$ \\
\hline Exit Then Re-Entrance onto Roadway & 0 & $0.0 \%$ & 4 & $3.4 \%$ & 4 & $2.8 \%$ \\
\hline Turn/Exit From Incorrect Lane & 1 & $4.0 \%$ & 3 & $2.6 \%$ & 4 & $2.8 \%$ \\
\hline $\begin{array}{l}\text { Late Braking For Stopped/ Stopping } \\
\text { Traffic }\end{array}$ & 3 & $12.0 \%$ & 0 & $0.0 \%$ & 3 & $2.1 \%$ \\
\hline Slow Upon Passing & 0 & $0.0 \%$ & 3 & $2.6 \%$ & 3 & $2.1 \%$ \\
\hline Turn Without Sufficient Warning & 0 & $0.0 \%$ & 2 & $1.7 \%$ & 2 & $1.4 \%$ \\
\hline Lateral Deviation Of Through Vehicle & 1 & $4.0 \%$ & 1 & $0.9 \%$ & 2 & $1.4 \%$ \\
\hline $\begin{array}{l}\text { Conflict Between Merging and Exiting } \\
\text { Traffic }\end{array}$ & 0 & $0.0 \%$ & 2 & $1.7 \%$ & 2 & $1.4 \%$ \\
\hline Following Too Closely & 1 & $4.0 \%$ & 0 & $0.0 \%$ & 1 & $0.7 \%$ \\
\hline Obscene Gesture (To Other Driver) & 1 & $4.0 \%$ & 0 & $0.0 \%$ & 1 & $0.7 \%$ \\
\hline Proceeding Through Red Traffic Signal & 1 & $4.0 \%$ & 0 & $0.0 \%$ & 1 & $0.7 \%$ \\
\hline
\end{tabular}




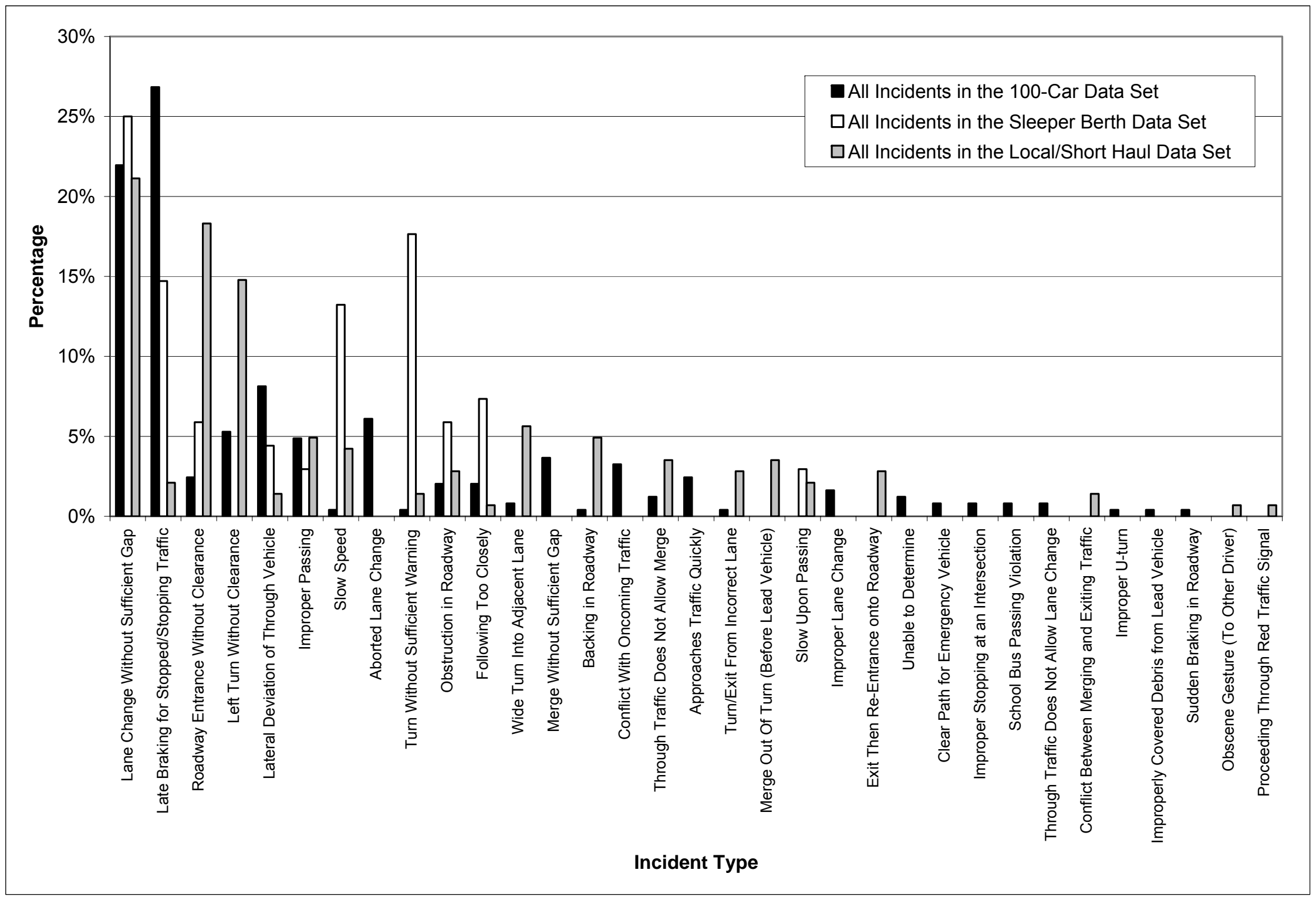

Figure 35. Percentage of Incident Types in the 100-Car, SB, L/SH Studies. 
Figure 36 shows the percentage of HV driver at-fault incidents in the 100-Car, SB, and L/SH studies as a function of Incident Type. The black bars in Figure 36 represent HV driver at-fault incidents in the 100-Car data set, while the white and grey bars represent SB and L/SH driver atfault incidents, respectively. The most frequent Incident Types for $\mathrm{HV}$ driver at-fault incidents in the 100-Car Study were Lane Change Without Sufficient Gap (26.6\%), Lateral Deviation of Through Vehicle (21.5\%), and Left Turn Without Clearance (13.9\%). The most frequent Incident Types for $\mathrm{HV}$ driver at-fault incidents in the SB study were Late Braking for Stopped/Stopping Traffic (47.6\%), Following Too Close (23.8\%), and Turn Without Sufficient Warning (14.3\%). The most frequent Incident Types for HV driver at-fault incidents in the L/SH study were Roadway Entrance Without Clearance (20\%), Backing In Roadway (12\%), Late Braking for Stopped/Stopping Traffic (12\%), Wide Turn Into Adjacent Lane (12\%), and Low Speed (8\%). 


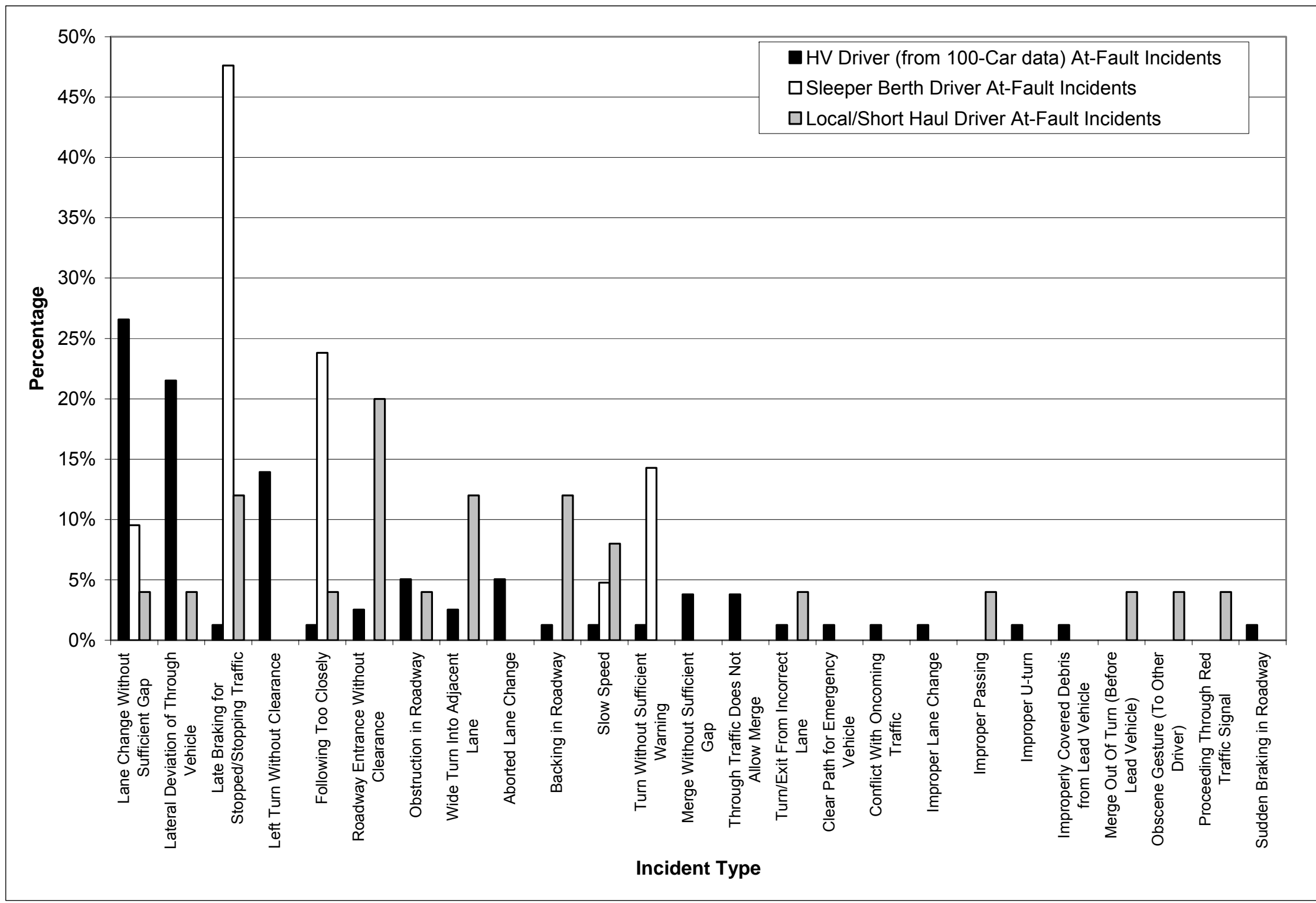

Figure 36. Percentage of HV Driver At-Fault Incident Types in the 100-Car, SB, and L/SH Studies. 
Figure 37 shows the percentage of LV driver at-fault incidents in the 100-Car, SB, and L/SH studies as a function of Incident Type. The black bars in Figure 37 represent the 100-Car driver at-fault incidents in the 100-Car Study, while the white and grey bars represent LV driver at-fault incidents in the SB and L/SH data sets, respectively. The most frequent Incident Types for LV driver at-fault incidents in the 100-Car Study were Late Braking for Stopped/Stopping Traffic (41.3\%) Lane Change Without Sufficient Gap (21.7\%), and Aborted Lane Change (8\%). The most frequent Incident Types for LV driver at-fault incidents in the SB study were Lane Change Without Sufficient Gap (32\%), Turn Without Sufficient Warning (19.1\%), and Low Speed (17\%). The most frequent Incident Types for LV driver at-fault incidents in the L/SH study were Lane Change Without Sufficient Gap (24.8\%), Roadway Entrance Without Clearance (17.9\%), Turn Without Clearance (17.9\%), and Improper Passing (5.1\%). 


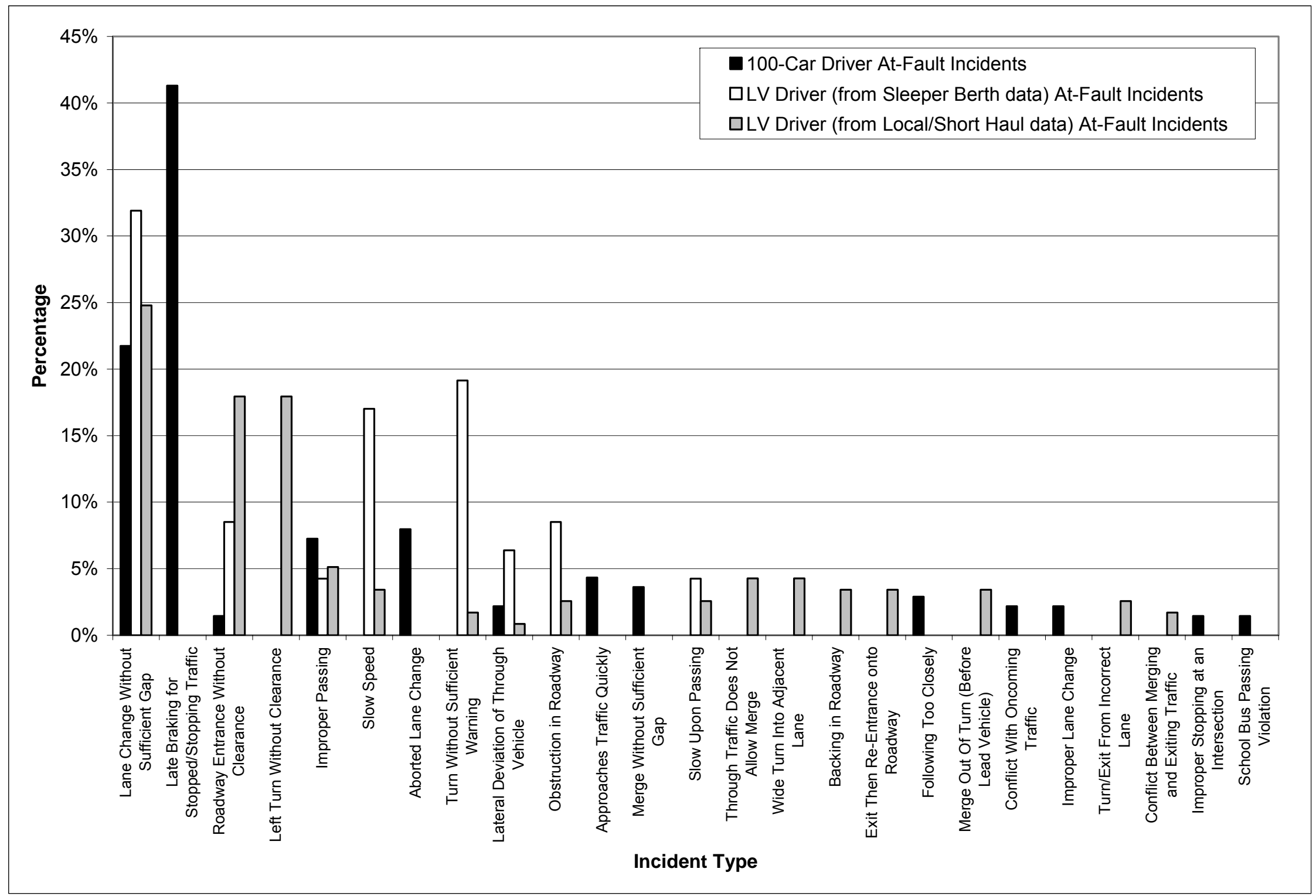

Figure 37. Percentage of LV Driver At-Fault Incident Types in the 100-Car, SB, and L/SH Studies. 
As can be seen in Figure 37, the Incident Types differed markedly depending on the study (i.e., 100-Car, SB, and L/SH). One possible explanation for these discrepancies could be the frequency of travel on certain Road Types (i.e., it would be expected that a significant amount of the incidents would occur on the most traveled roadways, similar to exposure). Of course, this is an oversimplification. The geographical area, what the vehicle was used for, and driver preference all dictate the predominant Road Type used in driving. Figure 38 illustrates the percentage of incidents for each of the three studies as a function of Road Type. The black bars in Figure 38 represent 100-Car incidents, while the white and grey bars represent SB and L/SH incidents, respectively. In the 100-Car data set, the highest proportion of incidents occurred on the Urban Divided (60.2\%), Urban Undivided (18.7\%), and Rural Undivided (9.3\%) roads. In the SB data set, the highest proportion of incidents occurred on the Rural Divided (55.9\%), Urban Undivided (10.3\%), and Rural Undivided (13.2\%) roads. In the L/SH data set, the highest proportion of incidents occurred on the Rural Divided (38.7\%), Urban Undivided (18.3\%), and Rural Undivided (16.2\%) roads. 


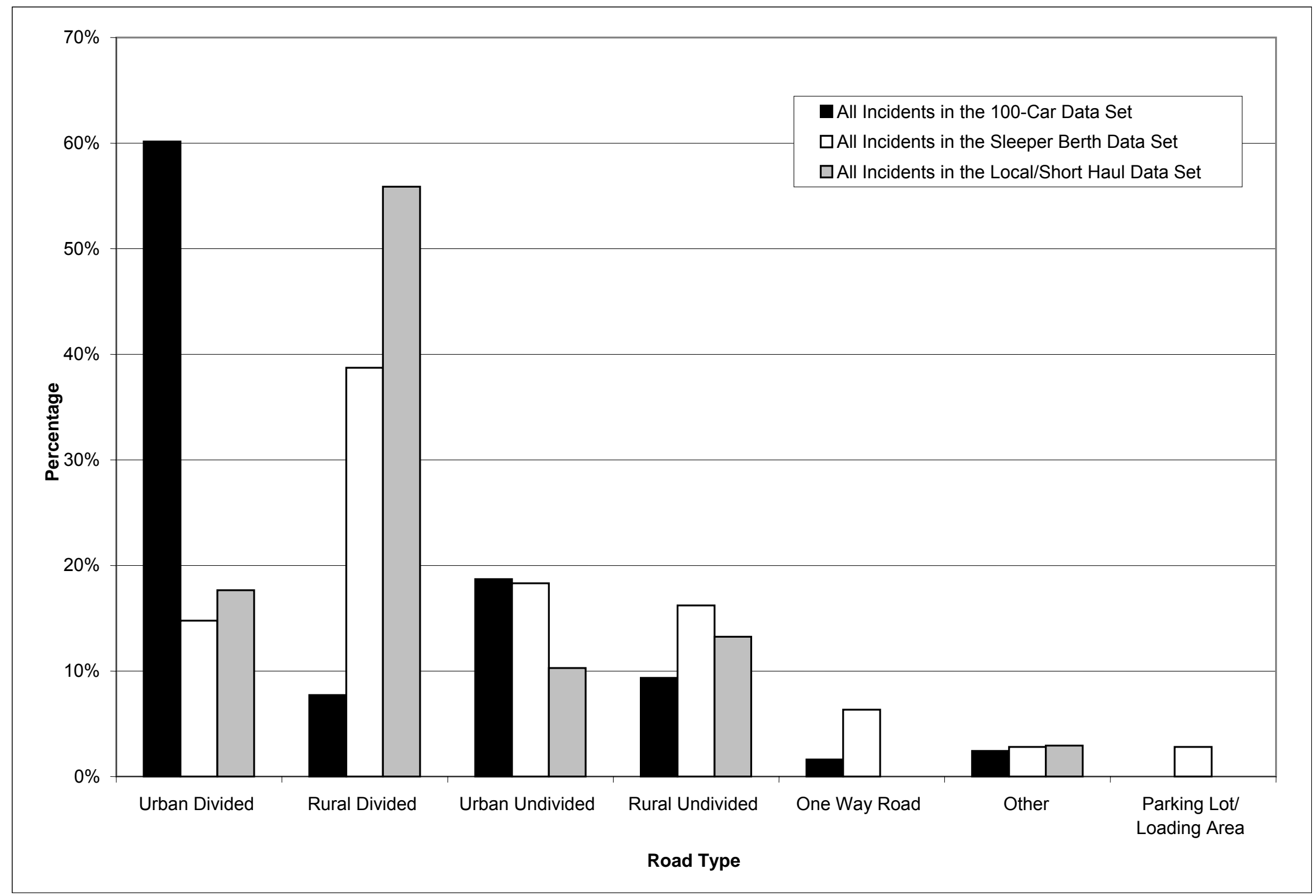

Figure 38. Percent of Incidents in the 100-Car, SB, and L/SH studies as a Function of Road Type. 


\section{Summary of Incident Types}

Across the three studies, the most frequent Incident Types were Lane Change Without Sufficient Gap (22.1\%), Late Braking for Stopped/Stopping Traffic, (17.3\%), and Roadway Entrance Without Clearance (7.9\%). These three Incident Types accounted for $47.3 \%$ of LV-HV interactions across the three studies.

As can be seen in Figure 36, the HV driver at-fault Incident Types differed with respect to the instrumented vehicle. The most frequent Incident Types for $\mathrm{HV}$ driver at-fault incidents in the 100-Car Study were Lane Change Without Sufficient Gap (26.6\%), Lateral Deviation of Through Traffic (21.5\%), and Left Turn Without Clearance (13.9\%). These three Incident Types represented $62 \%$ of the HV driver at-fault incidents in the 100-Car Study. The most frequent Incident Types for HV driver at-fault incidents in the SB study were Late Braking for Stopped/Stopping Traffic (47.6\%), Following Too Closely (23.8\%), and Turn Without Sufficient Warning (14.3\%). These three Incident Types represented $85.7 \%$ of the HV driver at-fault incidents in the SB study. The most frequent Incident Types for the HV driver at-fault incidents in the L/SH study were Roadway Entrance Without Clearance (20\%), Wide Turn Into Adjacent Area (12\%), and Backing in Roadway (12\%). These three Incident Types represented $48 \%$ of $\mathrm{HV}$ driver at-fault incident in the $\mathrm{L} / \mathrm{SH}$ study.

As can be seen in Figure 37, the LV driver at-fault Incident Types also differed with respect to the instrumented vehicle. The most frequent Incident Types for LV driver at-fault incidents in the 100-Car Study were Late Braking for Stopped/Stopping Traffic (41.3\%) and Lane Change Without Sufficient Gap (21.7\%). These two Incident Types represent $63 \%$ of the LV driver atfault incidents in the 100-Car Study. The most frequent Incident Types for LV driver at-fault incidents in the SB study were Lane Change Without Sufficient Gap (31.9\%), Turn Without Sufficient Warning (19.1\%), and Low Speed (17\%). These three Incident Types represent 68\% of the LV driver at-fault incidents in the SB study. The most frequent Incident Types for the LV driver at-fault incidents in the L/SH study were Lane Change Without Sufficient Gap (24.8\%), Roadway Entrance Without Clearance (17.9\%), and Left Turn Without Clearance (17.9\%). These three Incident Types represent $60.6 \%$ of LV driver at-fault incident in the L/SH study.

There were many differences across the three studies (100-Car, L/SH, and SB) as well between HVs and LVs within each study. This is possible as both the SB and L/SH trucks were instrumented in their respective studies, while the LV was instrumented in the 100-Car Study. Thus, those incidents recorded in the 100-Car Study reflect a diverse range of HVs. Further, the incidents in the SB and L/SH studies are likely to reflect difficulties specific to SB and L/SH operations. Support for this hypothesis was found when the authors assessed the location of each incident (see Figure 38).

Consider the Road Type comparison data shown in Figure 38. The bar graph illustrates the percentage of incidents as a function of different Road Types. Not surprisingly, the Road Types frequented by the 100-Car participants, such as major city roads and streets, are where the majority of 100-Car Study incidents occurred (60.2\% of the incidents occurred on an Urban Divided road). The Road Types common to SB operations, such as interstates and highways, are where the majority of SB incidents occurred. That is, rural divided by median (i.e., interstate) and urban divided by median (i.e., highway) accounted for $74 \%$ of the SB incidents. Similarly, 
the Road Types common for L/SH trucks accounted for many of the L/SH incidents. On a percentage basis, there were more incidents for L/SH drivers in town settings (i.e., urban undivided, urban divided, one-way, and parking lot), which is where many L/SH delivery routes

are located. By looking at the driving environments, it could be said that the majority of 100-Car and L/SH incidents occurred in and around town/urban areas (lower speeds and higher traffic areas). SB incidents, on the other hand, tended to occur on highways where speeds are relatively high and traffic density is relatively low.

For example, Roadway Entrance Without Clearance accounted for a substantial portion of L/SH incidents (18.3\%), whereas this Incident Type only accounted for a small proportion of the incidents in the 100-Car (2.4\%) and SB (5.9\%) studies. This makes intuitive sense when one considers L/SH operations trucking operations. L/SH drivers have many deliveries during their workday and will, therefore, routinely exit parking lots onto roadways. This provides an opportunity for this particular Incident Type to occur, whereas this maneuver is not characteristic of 100-Car and SB drivers. Much of the time, SB drivers were on limited-access highways having no intersecting side roads, whereas 100-Car drivers were driving on major urban road going to and from their residence. The characteristics of the Road Types traveled by 100-Car, $\mathrm{SB}$, and L/SH drivers appear to explain some of the discrepancies between the three studies.

\section{PRIMARY MANEUVERS}

Table 38 shows the frequency, percentage, and rank ordering of the Primary Maneuvers across the 100-Car, SB, and L/SH studies. The most frequent Primary Maneuver across all three studies was Changing Lanes (23.2\%), followed by Braking (12.3\%) and Left Turn (11.2\%). Figure 39 shows a bar graph of the 456 incidents, across the three studies, as a function of Primary Maneuver. 
Table 38. Frequency, percentage, and rank ordering of Primary Maneuvers across the three studies.

\begin{tabular}{|c|c|c|c|}
\hline Primary Maneuver & $\begin{array}{l}\text { Frequency of } \\
\text { Incidents Across } \\
\text { all Three Studies }\end{array}$ & $\begin{array}{l}\text { Percentage of } \\
\text { Incidents } \\
\text { Across all } \\
\text { Three Studies }\end{array}$ & Combined Rank \\
\hline Changing Lanes & 106 & $23.2 \%$ & 1 \\
\hline Braking & 56 & $12.3 \%$ & 2 \\
\hline Left Turn & 51 & $11.2 \%$ & 3 \\
\hline Through Traffic & 44 & $9.6 \%$ & 4 \\
\hline Stopped & 38 & $8.3 \%$ & 5 \\
\hline Traveling Ahead & 28 & $6.1 \%$ & 6 \\
\hline Right Turn & 26 & $5.7 \%$ & 7 \\
\hline Merging & 25 & $5.5 \%$ & 8 \\
\hline Crossing Over Lane Line & 19 & $4.2 \%$ & 9 \\
\hline Slower Speed & 15 & $3.3 \%$ & 10 \\
\hline Aborted Lane Change & 8 & $1.8 \%$ & 12.5 \\
\hline Enters Roadway & 8 & $1.8 \%$ & 12.5 \\
\hline U-Turn & 8 & $1.8 \%$ & 12.5 \\
\hline Roadway Exit & 8 & $1.8 \%$ & 12.5 \\
\hline Backing & 6 & $1.3 \%$ & 15 \\
\hline Avoiding Vehicle & 3 & $.7 \%$ & 17 \\
\hline Moved to Shoulder & 3 & $.7 \%$ & 17 \\
\hline Incomplete Lane Change & 2 & $.4 \%$ & 17 \\
\hline Drifts to the Left & 1 & $.2 \%$ & 19.5 \\
\hline Parked & 1 & $.2 \%$ & 19.5 \\
\hline
\end{tabular}




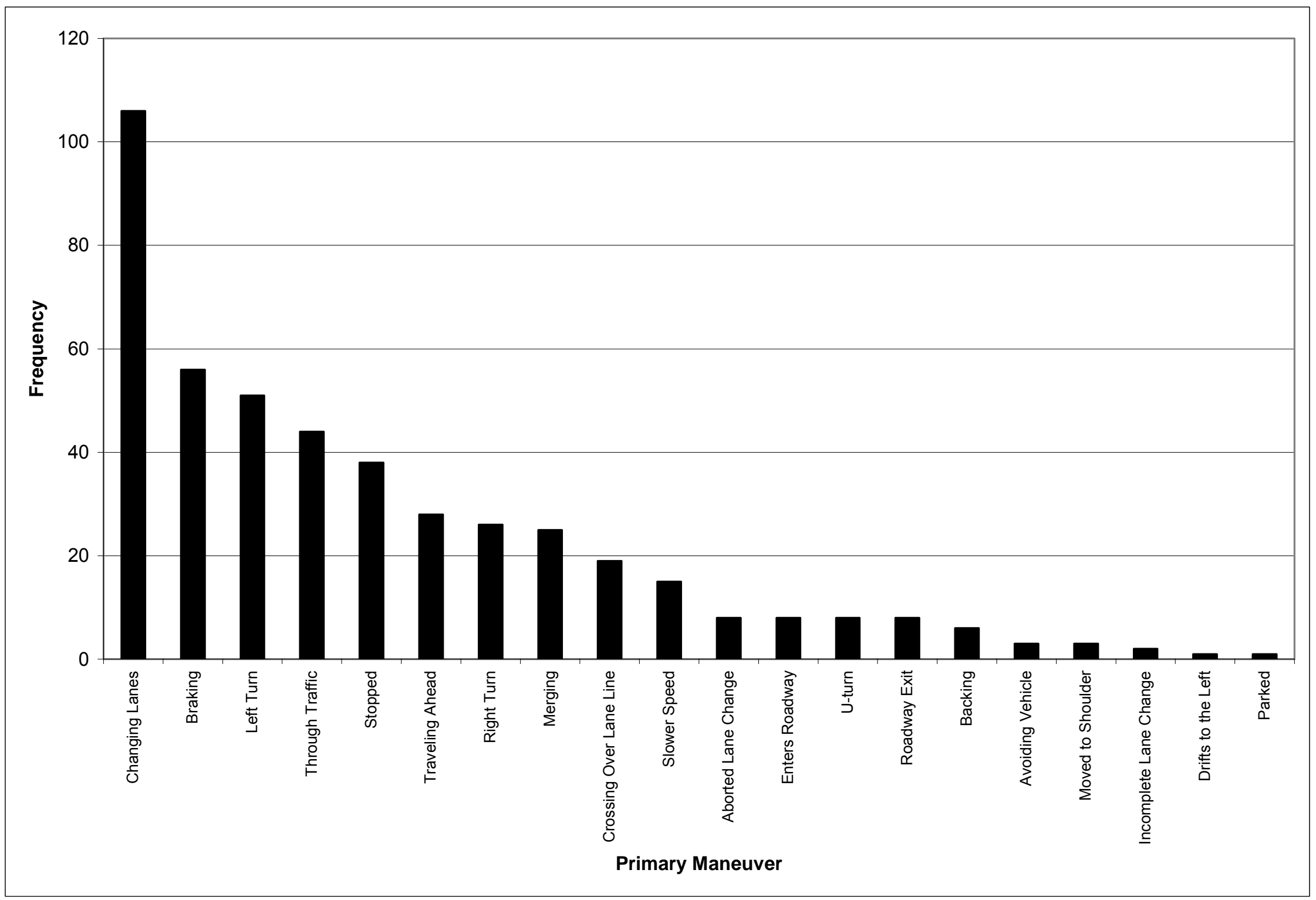

Figure 39. Frequency of Primary Maneuvers Across the Three Studies. 
Tables 39-41 show the frequency and percentage of each Primary Maneuver in the 100-Car, SB, and L/SH studies, respectively. In the 100-Car Study, the most frequent Primary Maneuvers were Braking (22.8\%), Changing Lanes (21.1\%), and Stopped (15\%). In the SB study, the most frequent Primary Maneuvers were Through Traffic (39.7\%), Changing Lanes (32.4\%), Roadway Exit (8.8\%), and Left Turn (8.8\%). In the L/SH study, the most frequent Primary Maneuvers were Changing Lanes (22.5\%), Left Turn (20.4\%), and Through Traffic (19\%). Figure 40 shows a bar graph illustrating the incidents for the 100-Car, SB, and L/SH studies as a function of Primary Maneuver. 
Table 39. Frequency and Percentage of Primary Maneuvers for the 100-Car Study.

\begin{tabular}{|c|c|c|c|c|c|c|c|c|}
\hline Primary Maneuver & $\begin{array}{c}\text { Frequency of } \\
\text { 100-Car Driver } \\
\text { At-Fault } \\
\text { Incidents } \\
\left(\mathrm{N}_{100-C a r}=138\right)\end{array}$ & $\begin{array}{c}\text { Percentage of } \\
\text { 100-Car Driver } \\
\text { At-Fault } \\
\text { Incidents } \\
\left(\mathrm{N}_{100-\text { Car }}=138\right)\end{array}$ & $\begin{array}{l}\text { Frequency of } \\
\text { HV Driver At- } \\
\text { Fault Incidents } \\
\left(\mathrm{N}_{\mathrm{HV}}=79\right)\end{array}$ & $\begin{array}{l}\text { Percentage of } \\
\text { HV Driver At- } \\
\text { Fault Incidents } \\
\left(\mathrm{N}_{\mathrm{HV}}=79\right)\end{array}$ & $\begin{array}{l}\text { Frequency of } \\
\text { Unknown } \\
\text { Driver At-Fault } \\
\text { Incidents } \\
\left(N_{U n}=29\right)\end{array}$ & $\begin{array}{l}\text { Percentage of } \\
\text { Unknown } \\
\text { Driver At-Fault } \\
\text { Incidents } \\
\left(\mathrm{N}_{\text {Un }}=29\right)\end{array}$ & $\begin{array}{l}\text { Frequency of } \\
\text { All 100-Car } \\
\text { Incidents } \\
\left(\mathrm{N}_{\text {Total }}=246\right)\end{array}$ & $\begin{array}{l}\text { Percentage of } \\
\text { All 100-Car } \\
\text { Incidents } \\
\left(\mathrm{N}_{\text {Total }}=246\right)\end{array}$ \\
\hline Braking & 45 & $32.6 \%$ & 3 & $3.8 \%$ & 8 & $27.6 \%$ & 56 & $22.8 \%$ \\
\hline Changing Lanes & 23 & $16.7 \%$ & 26 & $32.9 \%$ & 3 & $10.3 \%$ & 52 & $21.1 \%$ \\
\hline Stopped & 30 & $21.7 \%$ & 4 & $5.1 \%$ & 3 & $10.3 \%$ & 37 & $15.0 \%$ \\
\hline Crossing Over Lane Line & 2 & $1.4 \%$ & 16 & $20.3 \%$ & 1 & $3.4 \%$ & 19 & $7.7 \%$ \\
\hline Left Turn & 1 & $0.7 \%$ & 12 & $15.2 \%$ & 3 & $10.3 \%$ & 16 & $6.5 \%$ \\
\hline Through Traffic & 6 & $4.3 \%$ & 4 & $5.1 \%$ & 6 & $20.7 \%$ & 16 & $6.5 \%$ \\
\hline Slower Speed & 13 & $9.4 \%$ & 1 & $1.3 \%$ & 1 & $3.4 \%$ & 15 & $6.1 \%$ \\
\hline Aborted Lane Change & 7 & $5.1 \%$ & 1 & $1.3 \%$ & 0 & $0.0 \%$ & 8 & $3.3 \%$ \\
\hline Merging & 3 & $2.2 \%$ & 3 & $3.8 \%$ & 0 & $0.0 \%$ & 6 & $2.4 \%$ \\
\hline Right Turn & 3 & $2.2 \%$ & 2 & $2.5 \%$ & 1 & $3.4 \%$ & 6 & $2.4 \%$ \\
\hline Avoiding Vehicle & 3 & $2.2 \%$ & 0 & $0.0 \%$ & 0 & $0.0 \%$ & 3 & $1.2 \%$ \\
\hline Moved to Shoulder & 1 & $0.7 \%$ & 1 & $1.3 \%$ & 1 & $3.4 \%$ & 3 & $1.2 \%$ \\
\hline Enters Roadway & 0 & $0.0 \%$ & 1 & $1.3 \%$ & 1 & $3.4 \%$ & 2 & $0.8 \%$ \\
\hline Incomplete Lane Change & 0 & $0.0 \%$ & 1 & $1.3 \%$ & 1 & $3.4 \%$ & 2 & $0.8 \%$ \\
\hline Drifts to the Left & 1 & $0.7 \%$ & 0 & $0.0 \%$ & 0 & $0.0 \%$ & 1 & $0.4 \%$ \\
\hline Backing & 0 & $0.0 \%$ & 1 & $1.3 \%$ & 0 & $0.0 \%$ & 1 & $0.4 \%$ \\
\hline Parked & 0 & $0.0 \%$ & 1 & $1.3 \%$ & 0 & $0.0 \%$ & 1 & $0.4 \%$ \\
\hline Traveling Ahead & 0 & $0.0 \%$ & 1 & $1.3 \%$ & 0 & $0.0 \%$ & 1 & $0.4 \%$ \\
\hline U-Turn & 0 & $0.0 \%$ & 1 & $1.3 \%$ & 0 & $0.0 \%$ & 1 & $0.4 \%$ \\
\hline
\end{tabular}


Table 40. Frequency and Percentage of Primary Maneuvers for the Sleeper Berth Study.

\begin{tabular}{|c|c|c|c|c|c|c|}
\hline Primary Maneuver & $\begin{array}{l}\text { Frequency of SB } \\
\text { Driver At-Fault } \\
\text { Incidents } \\
\left(\mathrm{n}_{\mathrm{SB}}=21\right)\end{array}$ & $\begin{array}{l}\text { Percentage of } \\
\text { SB Driver At- } \\
\text { Fault Incidents } \\
\left(\mathrm{n}_{\mathrm{SB}}=21\right)\end{array}$ & $\begin{array}{l}\text { Frequency of } \\
\text { LV Driver At- } \\
\text { Fault Incidents } \\
\quad\left(\mathrm{n}_{\mathrm{LV}}=47\right)\end{array}$ & $\begin{array}{l}\text { Percentage of } \\
\text { LV Driver At- } \\
\text { Fault Incidents } \\
\left(\mathrm{n}_{\mathrm{LV}}=47\right)\end{array}$ & $\begin{array}{c}\text { Frequency of } \\
\text { All SB } \\
\text { Incidents } \\
\left(n_{\text {Total }}=68\right)\end{array}$ & $\begin{array}{c}\text { Percentage of } \\
\text { All SB } \\
\text { Incidents }\end{array}$ \\
\hline Through Traffic & 15 & $71.4 \%$ & 12 & $25.5 \%$ & 27 & $39.7 \%$ \\
\hline Changing Lanes & 2 & $9.5 \%$ & 20 & $42.6 \%$ & 22 & $32.4 \%$ \\
\hline Left Turn & 2 & $9.5 \%$ & 4 & $8.5 \%$ & 6 & $8.8 \%$ \\
\hline Roadway Exit & 1 & $4.8 \%$ & 5 & $10.6 \%$ & 6 & $8.8 \%$ \\
\hline Merge Onto Roadway & 0 & $0 \%$ & 4 & $8.5 \%$ & 4 & $5.9 \%$ \\
\hline Right Turn & 1 & $4.8 \%$ & 1 & $3.7 \%$ & 2 & $2.9 \%$ \\
\hline Stopped in Roadway & 0 & $0 \%$ & 1 & $3.7 \%$ & 1 & $1.5 \%$ \\
\hline Backing & 0 & $0 \%$ & 0 & $0 \%$ & 0 & $0 \%$ \\
\hline Roadway Entrance & 0 & $0 \%$ & 0 & $0 \%$ & 0 & $0 \%$ \\
\hline U-Turn & 0 & $0 \%$ & 0 & $0 \%$ & 0 & $0 \%$ \\
\hline
\end{tabular}


Table 41. Frequency and Percentage of Primary Maneuvers for the Local/Short Haul Study.

\begin{tabular}{|c|c|c|c|c|c|c|}
\hline Primary Maneuver & $\begin{array}{l}\text { Frequency of } \\
\text { L/SH Driver At- } \\
\text { Fault Incidents } \\
\left(n_{\mathrm{L} / \mathrm{SH}}=25\right)\end{array}$ & $\begin{array}{l}\text { Percentage of } \\
\text { L/SH Driver At- } \\
\text { Fault Incidents } \\
\left(n_{\mathrm{L} / \mathrm{SH}}=25\right)\end{array}$ & $\begin{array}{l}\text { Frequency of } \\
\text { LV Driver At- } \\
\text { Fault Incidents } \\
\left(\mathrm{n}_{\mathrm{LV}}=117\right)\end{array}$ & $\begin{array}{l}\text { Percentage of } \\
\text { LV Driver At- } \\
\text { Fault Incidents } \\
\left(\mathrm{n}_{\mathrm{LV}}=117\right)\end{array}$ & $\begin{array}{l}\text { Frequency of } \\
\text { All L/SH } \\
\text { Incidents } \\
\left(\mathrm{N}_{\text {Total }}=142\right)\end{array}$ & $\begin{array}{c}\text { Percentage of } \\
\text { All L/SH } \\
\text { Incidents } \\
\left(\mathrm{N}_{\text {Total }}=142\right)\end{array}$ \\
\hline Changing Lanes & 1 & $4 \%$ & 31 & $26.5 \%$ & 32 & $22.5 \%$ \\
\hline Left Turn & 3 & $12 \%$ & 26 & $22.2 \%$ & 29 & $20.4 \%$ \\
\hline Through Traffic & 11 & $44 \%$ & 16 & $13.7 \%$ & 27 & $19 \%$ \\
\hline Right Turn & 6 & $24 \%$ & 12 & $10.3 \%$ & 18 & $12.7 \%$ \\
\hline Merge Onto Roadway & 1 & $4 \%$ & 14 & $12 \%$ & 15 & $10.6 \%$ \\
\hline U-Turn & 2 & $8 \%$ & 5 & $4.3 \%$ & 7 & $4.9 \%$ \\
\hline Roadway Entrance & 0 & $0 \%$ & 6 & $5.1 \%$ & 6 & $4.2 \%$ \\
\hline Backing & 1 & $4 \%$ & 4 & $3.4 \%$ & 5 & $3.5 \%$ \\
\hline Roadway Exit & 0 & $0 \%$ & 2 & $1.7 \%$ & 2 & $1.4 \%$ \\
\hline Stopped in Roadway & 0 & $0 \%$ & 1 & $.9 \%$ & 1 & $.7 \%$ \\
\hline
\end{tabular}




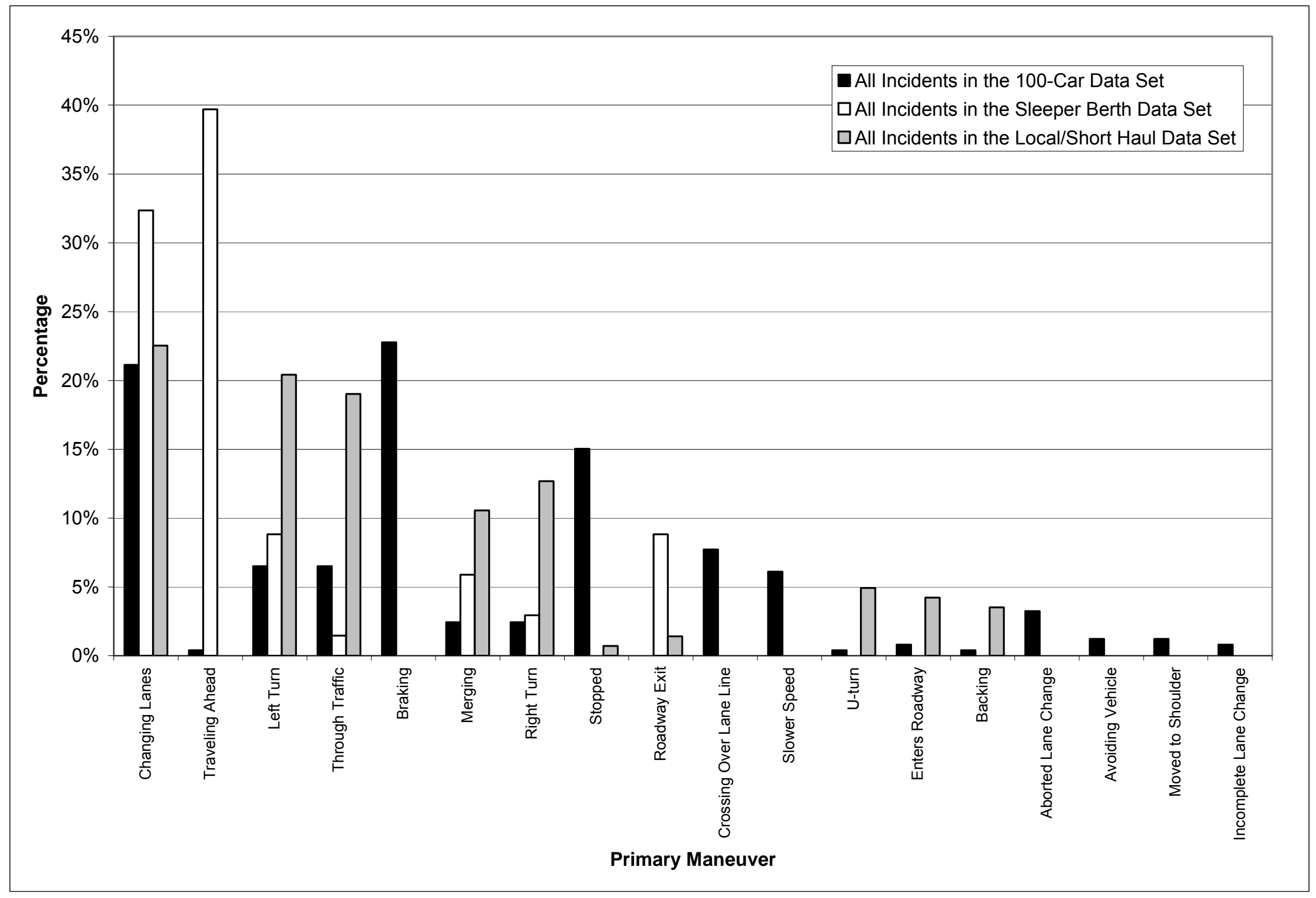

Figure 40. Percentage of Primary Maneuvers in the 100-Car, SB, and L/SH Studies. 
Figure 41 shows the percentage of HV driver at-fault incidents in the 100-Car, SB, and L/SH studies as a function of Primary Maneuver. The black bars in Figure 41 represent the HV driver at-fault incidents in the 100-Car Study, while the white and grey bars represent HV driver atfault incidents in the SB and L/SH studies, respectively. The most frequent Primary Maneuvers for HV driver at-fault incidents in the 100-Car Study were Changing Lanes (32.9\%), Crossing Over Lane Line (26.3\%), and Left Turn (15.2\%). The most frequent Primary Maneuvers for HV driver at-fault incidents in the SB study were Through Traffic (71.4\%), Changing Lanes (9.5\%), and Left Turn (9.5\%). The most frequent Primary Maneuvers for HV driver at-fault incidents in the L/SH study were Through Traffic (44\%), Right Turn (24\%), and Left Turn (12\%). 


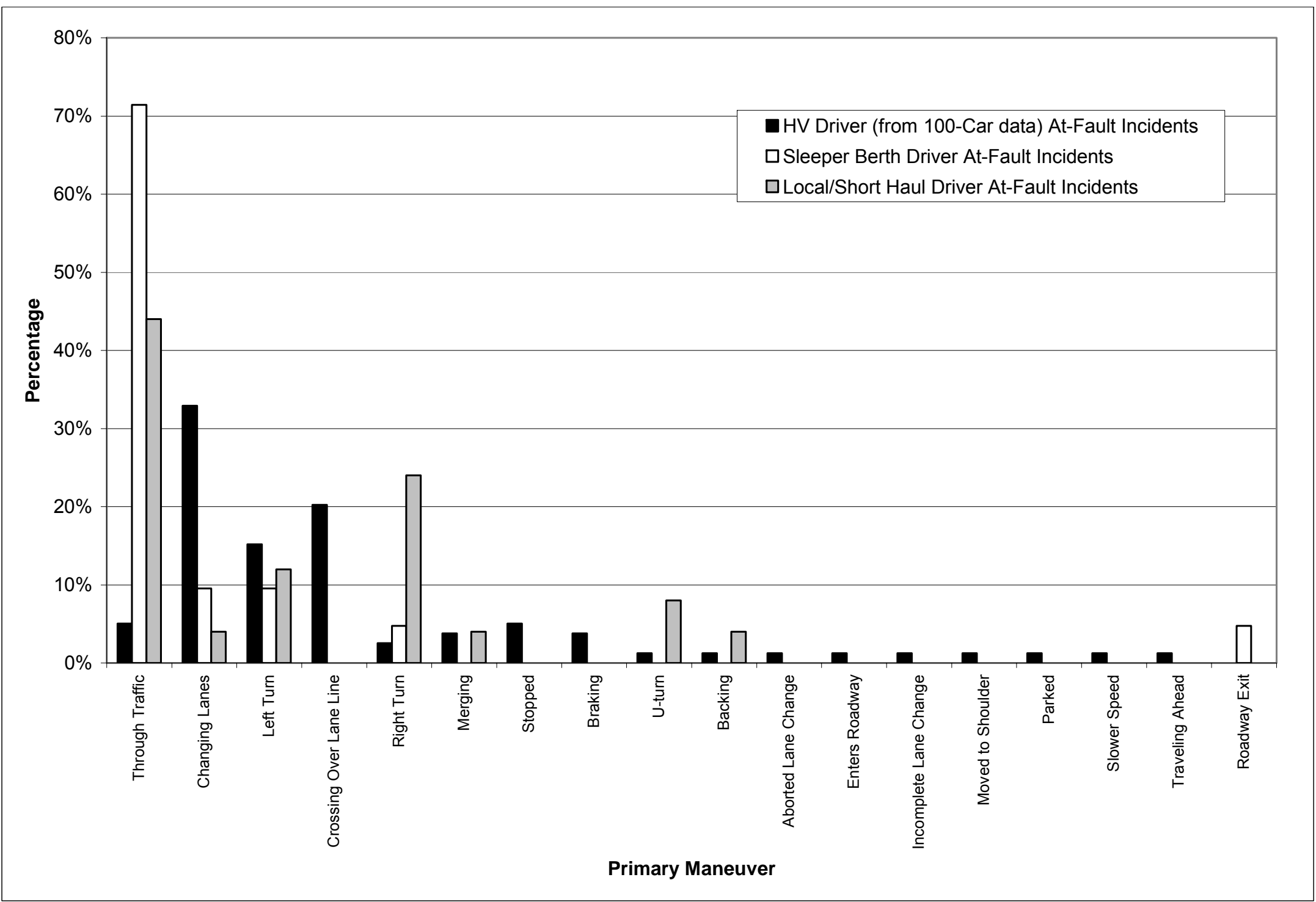

Figure 41. Percentage of HV Driver At-Fault Incidents in the 100-Car, SB, and L/SH Studies as a Function of Primary Maneuver. 
Figure 42 shows the percentage of LV driver at-fault incidents in the 100-Car, SB, and L/SH studies as a function of Primary Maneuver. The black bars in Figure 42 represent the LV driver at-fault incidents in the 100-Car Study, while the white and grey bars represent LV driver at-fault incidents in the SB and L/SH studies, respectively. The most frequent Primary Maneuvers for LV driver at-fault incidents in the 100-Car Study were Braking (32.6\%), Stopped (21.7\%), and Changing Lanes (16.7\%). The most frequent Primary Maneuvers for LV driver at-fault incidents in the SB study were Changing Lanes (42.6\%), Through Traffic (25.5\%), and Roadway Exit (10.6\%). The most frequent Primary Maneuvers for LV driver at-fault incidents in the L/SH study were Changing Lanes (26.5\%), Left Turn (22.2\%), and Through Traffic (13.7\%). 


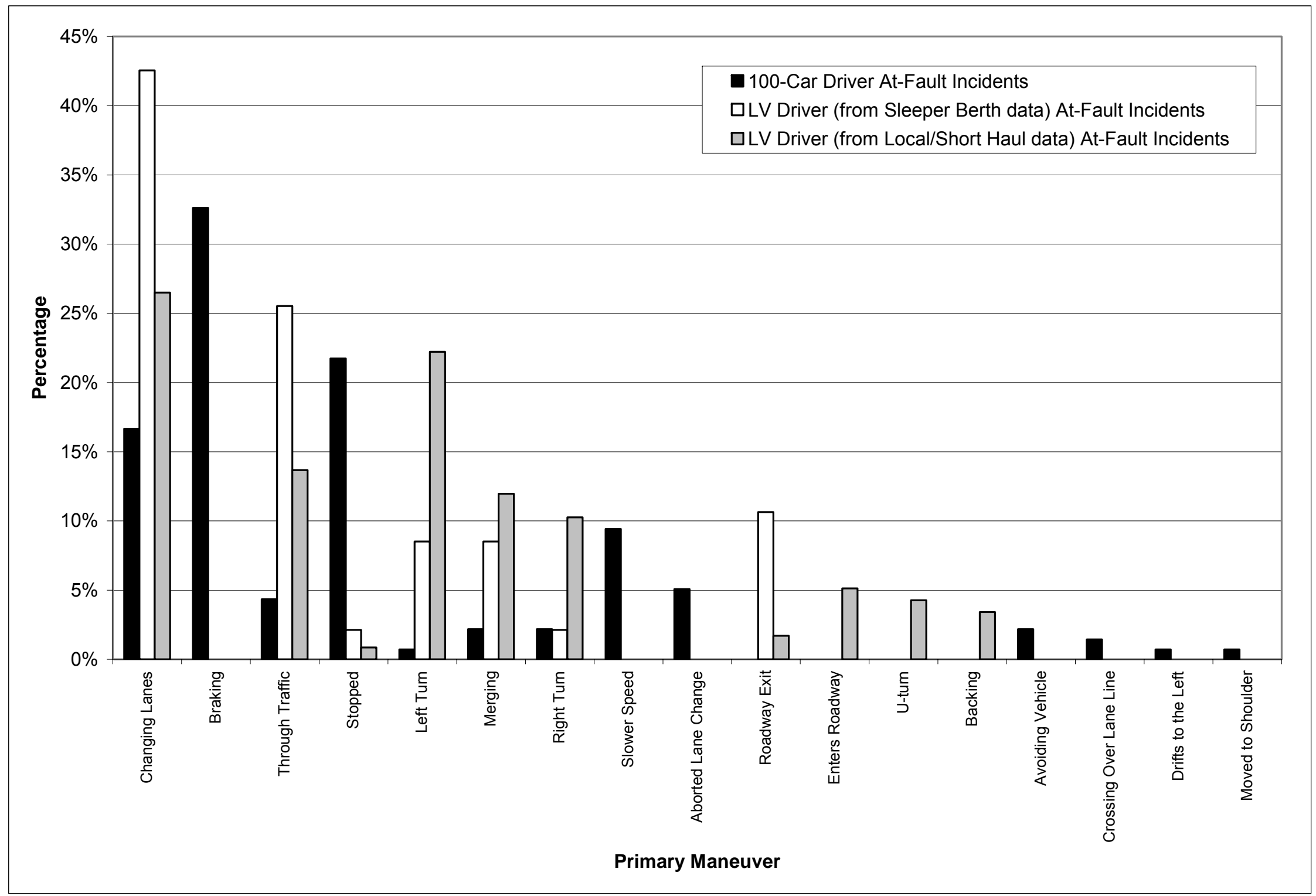

Figure 42. Percentage of LV Driver At-Fault Incidents in the 100-Car, SB, and L/SH Studies as a Function of Primary Maneuver. 


\section{Summary of Primary Maneuvers Across Studies}

Across the three studies, the most frequent Primary Maneuvers were Changing Lanes (23.2\%), Braking (12.3\%), and Left Turn (11.2\%). These three Primary Maneuvers represented 46.5\% of the Primary Maneuvers across the three studies. As can be seen in Figures 40-42, the Primary Maneuvers differed depending on whether the LV or HV was judged to have been at fault.

As can be seen in Figure 41, the most frequent Primary Maneuvers for HV driver at-fault incidents in the 100-Car Study were Changing Lanes (32.9\%), Crossing Over the Lane Line (26.3\%), and Left Turn (15.2\%). These three Primary Maneuvers represented $74.4 \%$ of the HV driver at-fault incidents in the 100-Car Study. In the SB study, the most frequent Primary Maneuvers for $\mathrm{HV}$ driver at-fault incidents were Through Traffic (71.4\%), Changing Lanes (9.5\%), and Left Turn (9.5\%). These three Primary Maneuvers accounted for $90.4 \%$ of the HV driver at-fault incident in the SB study. In the L/SH study, the most frequent Primary Maneuvers for HV driver at-fault incidents were Through Traffic (44\%), Right Turn (24\%), and Left Turn (12\%). These three Primary Maneuvers accounted for $80 \%$ of the HV driver at-fault incidents in the L/SH study. From the data it appears that HV drivers had the most difficulties when traveling forward on a roadway or straight through an intersection and changing lanes.

As can be seen in Figure 42, the most frequent Primary Maneuvers for LV driver at-fault incidents in the 100-Car Study were Braking (32.6\%), Stopped (21.7\%), and Changing Lanes (16.7\%). These three Primary Maneuvers accounted for $71 \%$ of the LV driver at-fault incidents in the 100-Car Study. In the SB study, the most frequent Primary Maneuvers for LV driver atfault incidents were Changing Lanes (42.6\%), Through Traffic (25.5\%), and Roadway Exit (10.6\%). These three Primary Maneuvers represented $78.7 \%$ of the LV driver at-fault incidents in the SB study. In the L/SH study, the most frequent Primary Maneuvers for LV driver at-fault incidents were Changing Lanes (26.5\%), Left Turn (22.2\%), and Through Traffic (13.7\%). These three Primary Maneuvers accounted for $62.4 \%$ of the LV driver at-fault incidents in the L/SH study. From the data it appears that LV drivers had the most difficulties when braking or decelerating and changing lanes.

In the SB study, the Through Traffic (i.e., vehicle traveling forward on a roadway or straight through an intersection) and Changing Lanes Primary Maneuvers accounted for $72 \%$ of the total incidents recorded. The Primary Maneuvers associated with LV-HV interactions in the L/SH and 100-Car studies were more varied. Changing Lanes (22.5\%), Left Turn (20.4\%), Through Traffic (19\%), Right Turn (12.7\%), and Merge onto Roadway (10.6\%) accounted for the majority of incidents recorded in the L/SH study, while Braking (22.8\%), Changing Lanes (21.1\%), Stopped (15\%), and Crossing Over Lane Line (7.7\%) represented the majority of Primary Maneuvers in the 100-Car Study. Further investigation of these maneuver types indicated that Through Primary Maneuver was the most frequent type for HVs in both the SB and L/SH studies: $71 \%$ and $44 \%$, respectively. However, Changing Lanes (32.9\%) was the most predominant Primary Maneuver for HVs in the 100-Car Study.

The Changing Lanes Primary Maneuver was the predominant type for LVs in both the SB and L/SH studies: $42.6 \%$ and 26.5\%, respectively. However, in the 100-Car Study, the Braking (32.6\%) Primary Maneuver was the most predominant type for LVs. Note that the predominant Primary Maneuvers for each group of drivers is consistent with the Incident Type classification 
presented in the previous section. For example, one would expect that the Primary Maneuver for Late Braking for Stopped/Stopping Traffic would be Through Traffic, which as indicated by the SB at-fault incidents, was the case.

\section{SPECIFIC CONTRIBUTING FACTORS}

Table 42 shows the frequency, percentage, and rank ordering of the Contributing Factors across all three studies. The most frequent Contributing Factor across the three studies was Driving Techniques (41\%), followed by Aggressive Driving (24.1\%), Unknown (17.1\%), and Distracted (10.5\%). The reader should bear in mind that more than one Contributing Factor could be selected for a single incident in the 100-Car Study. Figure 43 shows a bar graph of the 456 incidents, across the three studies, as a function of the Contributing Factors.

Table 42. Frequency, Percentage, and Rank Ordering of the Contributing Factors across the 100-Car, SB, and L/SH Studies $\left(n_{\text {Total }}=456\right)$.

\begin{tabular}{|l|c|c|c|}
\hline Specific Contributing Factor Category & $\begin{array}{c}\text { Frequency of } \\
\text { Incidents } \\
\text { Across all } \\
\text { Three } \\
\text { Studies }\end{array}$ & $\begin{array}{c}\text { Percentage of } \\
\text { Incidents } \\
\text { Across all } \\
\text { Three Studies }\end{array}$ & $\begin{array}{c}\text { Combined } \\
\text { Rank }\end{array}$ \\
\hline Driving Techniques & 187 & $41 \%$ & 1 \\
\hline Aggressive Driving & 110 & $24.1 \%$ & 2 \\
\hline Unknown & 78 & $17.1 \%$ & 3 \\
\hline Distracted & 48 & $10.5 \%$ & 4 \\
\hline Roadway Alignment & 26 & $5.7 \%$ & 5 \\
\hline Purposeful Violation of Traffic Laws & 15 & $3.3 \%$ & 6 \\
\hline Drowsy & 11 & $2.4 \%$ & 7 \\
\hline Vehicle Kinematics, Physics & 8 & $1.8 \%$ & 8 \\
\hline Roadway Sight Distance & 7 & $1.5 \%$ & 9 \\
\hline Driver Capabilities and Limitations & 6 & $1.3 \%$ & 10 \\
\hline Angry & 2 & $0.4 \%$ & 12 \\
\hline Other Emotional State & 2 & $0.4 \%$ & 12 \\
\hline Unfamiliar With Roadway/ Traffic Pattern & 2 & $0.4 \%$ & 12 \\
\hline Other & 1 & $0.2 \%$ & 15 \\
\hline Roadway Delineation & 1 & $0.2 \%$ & 15 \\
\hline Use of Vehicle For Improper Purposes & 1 & $0.2 \%$ & 15 \\
\hline
\end{tabular}




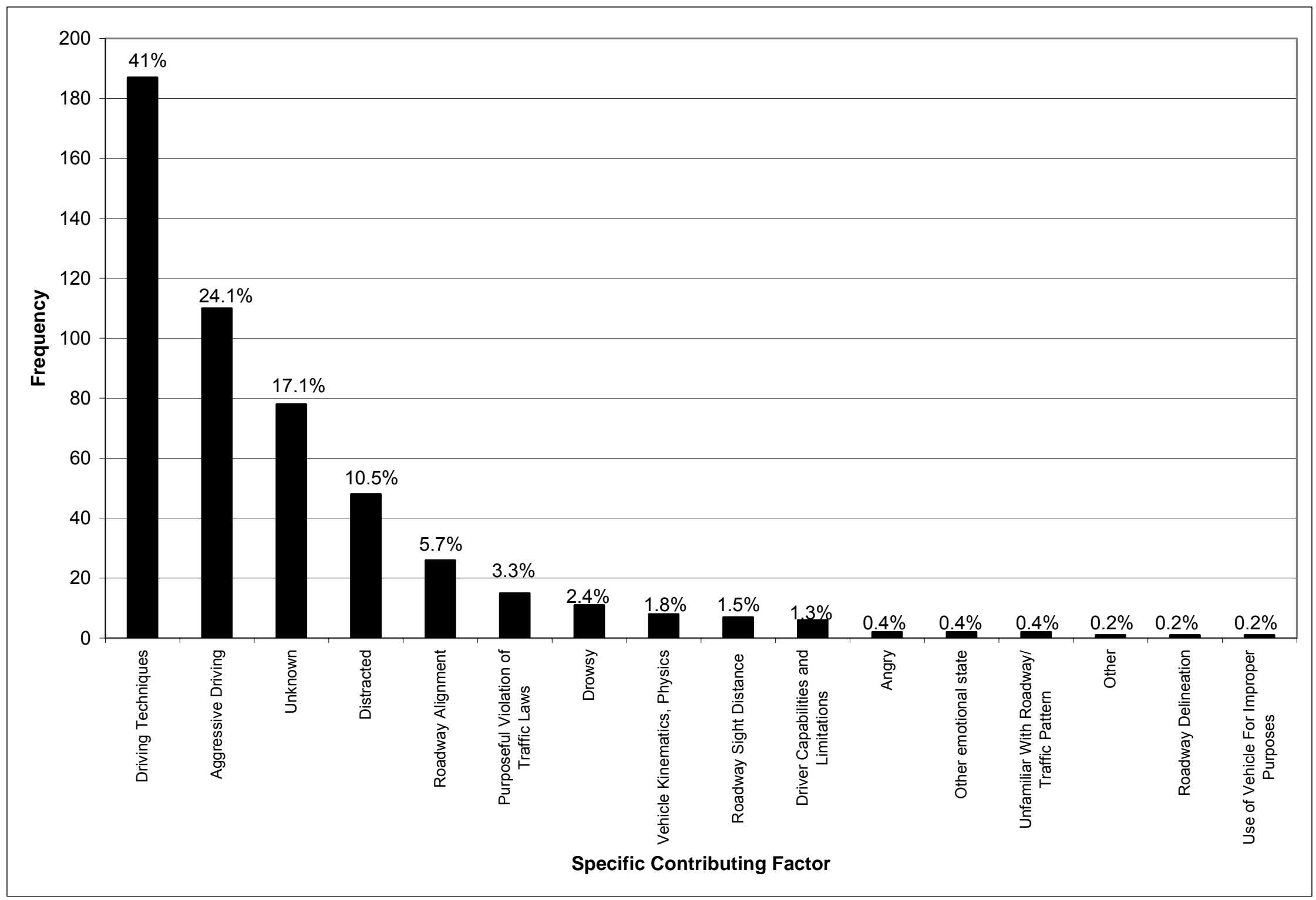

Figure 43. Frequency of Contributing Factors across the 100-Car, SB, and L/SH Studies. 
Tables 43-45 show the frequency and percentage of each Contributing Factor in the 100-Car, SB, and L/SH studies, respectively. In the 100-Car Study, the most frequent Contributing Factors were Driving Techniques (49.5\%), Unknown (24\%), Distracted (18.7\%), and Aggressive Driving (15\%). In the SB data set, the most frequent Contributing Factors were Driving Techniques (46\%) and Aggressive Driving (35\%). In the L/SH study, the most frequent Contributing Factors were Aggressive Driving (37\%), Driving Techniques (24\%), Roadway Alignment (11\%), and Unknown (11\%). Figure 44 shows a bar graph illustrating the 456 incidents in the 100-Car, SB, and L/SH studies as a function of the Contributing Factors. 
Table 43. Frequency and Percentage of Contributing Factors for the 100-Car Study.

\begin{tabular}{|c|c|c|c|c|c|c|c|c|}
\hline $\begin{array}{c}\text { Specific } \\
\text { Contributing Factor }\end{array}$ & $\begin{array}{l}\text { Frequency of } \\
\text { LV At-Fault } \\
\text { Incidents } \\
\left(n_{100 C a r}=138\right)\end{array}$ & $\begin{array}{l}\text { Percentage of } \\
\text { LV At-Fault } \\
\text { Incidents } \\
\left(\mathrm{n}_{100 \mathrm{Car}}=138\right)\end{array}$ & $\begin{array}{c}\text { Frequency of } \\
\text { HV Driver At- } \\
\text { Fault } \\
\text { Incidents } \\
\left(\mathbf{n}_{\mathrm{HV}}=79\right)\end{array}$ & $\begin{array}{l}\text { Percentage of } \\
\text { HV Driver At- } \\
\text { Fault } \\
\text { Incidents } \\
\left(\mathrm{n}_{\mathrm{HV}}=79\right)\end{array}$ & $\begin{array}{l}\text { Frequency of } \\
\text { Unknown } \\
\text { Driver At- } \\
\text { Fault } \\
\text { Incidents } \\
\text { (nUn = 29) }\end{array}$ & $\begin{array}{l}\text { Percentage of } \\
\text { Unknown } \\
\text { Driver At- } \\
\text { Fault } \\
\text { Incidents } \\
\text { (nun }=29)\end{array}$ & $\begin{array}{c}\text { Frequency of } \\
\text { All Drivers } \\
\left(\mathrm{N}_{\text {Total }}=246\right)\end{array}$ & $\begin{array}{c}\text { Percentage of } \\
\text { All Drivers } \\
\left(N_{\text {Total }}=246\right)\end{array}$ \\
\hline Driving Techniques & 96 & $54.2 \%$ & 12 & $14.5 \%$ & 14 & $40 \%$ & 122 & $49.5 \%$ \\
\hline Unknown & 0 & $0 \%$ & 54 & $65.1 \%$ & 5 & $14.3 \%$ & 59 & $24 \%$ \\
\hline Distracted & 31 & $18.6 \%$ & 9 & $10.8 \%$ & 6 & $17.1 \%$ & 46 & $18.7 \%$ \\
\hline Aggressive Driving & 31 & $17.5 \%$ & 3 & $1.2 \%$ & 3 & $2.9 \%$ & 37 & $15 \%$ \\
\hline Drowsy & 5 & $2.8 \%$ & 2 & $2.4 \%$ & 2 & $5.7 \%$ & 9 & $3.7 \%$ \\
\hline $\begin{array}{l}\text { Purposeful Violation of } \\
\text { Traffic Laws }\end{array}$ & 7 & $4 \%$ & 0 & $0 \%$ & 1 & $2.9 \%$ & 8 & $3.3 \%$ \\
\hline Roadway Alignment & 3 & $1.7 \%$ & 2 & $2.4 \%$ & 3 & $5.7 \%$ & 8 & $3.3 \%$ \\
\hline Roadway Delineation & 0 & $0 \%$ & 0 & $0 \%$ & 3 & $8.6 \%$ & 3 & $1.2 \%$ \\
\hline Angry & 0 & $0 \%$ & 1 & $1.2 \%$ & 1 & $2.9 \%$ & 2 & $.8 \%$ \\
\hline Other Emotional State & 1 & $.6 \%$ & 1 & $1.2 \%$ & 0 & $0 \%$ & 2 & $.8 \%$ \\
\hline Other & 1 & $.6 \%$ & 0 & $0 \%$ & 0 & $0 \%$ & 1 & $.4 \%$ \\
\hline $\begin{array}{l}\text { Vehicle Kinematics, } \\
\text { Physics }\end{array}$ & 0 & $0 \%$ & 0 & $0 \%$ & 0 & $0 \%$ & 0 & $0 \%$ \\
\hline $\begin{array}{l}\text { Driver Capabilities and } \\
\text { Limitations }\end{array}$ & 0 & $0 \%$ & 0 & $0 \%$ & 0 & $0 \%$ & 0 & $0 \%$ \\
\hline $\begin{array}{l}\text { Unfamiliar With } \\
\text { Roadway/ Traffic } \\
\text { Pattern }\end{array}$ & 0 & $0 \%$ & 0 & $0 \%$ & 0 & $0 \%$ & 0 & $0 \%$ \\
\hline $\begin{array}{l}\text { Roadway Sight } \\
\text { Distance }\end{array}$ & 0 & $0 \%$ & 0 & $0 \%$ & 0 & $0 \%$ & 0 & $0 \%$ \\
\hline $\begin{array}{l}\text { Use of Vehicle For } \\
\text { Improper Purposes }\end{array}$ & 0 & $0 \%$ & 0 & $0 \%$ & 0 & $0 \%$ & 0 & $0 \%$ \\
\hline
\end{tabular}


Table 44. Frequency and Percentage of Contributing Factors for the Sleeper Berth Study.

\begin{tabular}{|c|c|c|c|c|c|c|}
\hline $\begin{array}{l}\text { Specific Contributing } \\
\text { Factor Category }\end{array}$ & $\begin{array}{c}\text { Frequency of } \\
\text { SB Driver At- } \\
\text { Fault } \\
\text { Incidents } \\
\left(\mathrm{n}_{\mathrm{SB}}=21\right)\end{array}$ & $\begin{array}{l}\text { Percentage of } \\
\text { SB Driver At- } \\
\text { Fault } \\
\text { Incidents } \\
\left(\mathbf{n}_{\mathrm{SB}}=21\right)\end{array}$ & $\begin{array}{l}\text { Frequency of } \\
\text { LV Driver At- } \\
\text { Fault } \\
\text { Incidents } \\
\left(\mathrm{n}_{\mathrm{LV}}=47\right)\end{array}$ & $\begin{array}{l}\text { Percentage of } \\
\text { LV Driver At- } \\
\text { Fault } \\
\text { Incidents } \\
\left(\mathrm{n}_{\mathrm{LV}}=47\right)\end{array}$ & $\begin{array}{c}\text { Frequency of } \\
\text { All Drivers } \\
\left(\mathrm{N}_{\text {Total }}=68\right)\end{array}$ & $\begin{array}{c}\text { Percentage of } \\
\text { All Drivers } \\
\left(\mathrm{N}_{\text {Total }}=68\right)\end{array}$ \\
\hline Driving Techniques & 11 & $52 \%$ & 20 & $42.6 \%$ & 31 & $45.6 \%$ \\
\hline Aggressive Driving & 5 & $24 \%$ & 19 & $40.4 \%$ & 24 & $35.3 \%$ \\
\hline Roadway Alignment & 0 & $0 \%$ & 4 & $8.5 \%$ & 4 & $5.9 \%$ \\
\hline Unknown & 0 & $0 \%$ & 4 & $8.5 \%$ & 4 & $5.9 \%$ \\
\hline $\begin{array}{l}\text { Vehicle Kinematics, } \\
\text { Physics }\end{array}$ & 3 & $14 \%$ & 0 & $0 \%$ & 3 & $4.5 \%$ \\
\hline $\begin{array}{l}\text { Driver Capabilities and } \\
\text { Limitations }\end{array}$ & 1 & $5 \%$ & 0 & $0 \%$ & 1 & $1.5 \%$ \\
\hline Fatigue and Drowsiness & 1 & $5 \%$ & 0 & $0 \%$ & 1 & $1.5 \%$ \\
\hline $\begin{array}{l}\text { Purposeful Violation of } \\
\text { Traffic Laws }\end{array}$ & 0 & $0 \%$ & 0 & $0 \%$ & 0 & $0 \%$ \\
\hline Roadway Sight Distance & 0 & $0 \%$ & 0 & $0 \%$ & 0 & $0 \%$ \\
\hline $\begin{array}{l}\text { Unfamiliar With } \\
\text { Roadway/ Traffic Pattern }\end{array}$ & 0 & $0 \%$ & 0 & $0 \%$ & 0 & $0 \%$ \\
\hline $\begin{array}{l}\text { Use of Vehicle For } \\
\text { Improper Purposes }\end{array}$ & 0 & $0 \%$ & 0 & $0 \%$ & 0 & $0 \%$ \\
\hline
\end{tabular}


Table 45. Frequency and Percentage of Contributing Factors for the Local/Short Haul Study.

\begin{tabular}{|c|c|c|c|c|c|c|}
\hline $\begin{array}{l}\text { Specific Contributing } \\
\text { Factor Category }\end{array}$ & $\begin{array}{l}\text { Frequency of } \\
\text { L/SH Driver } \\
\text { At-Fault } \\
\text { Incidents } \\
\left(\mathrm{n}_{\mathrm{L} / \mathrm{SH}}=25\right)\end{array}$ & $\begin{array}{l}\text { Percentage of } \\
\text { L/SH Driver } \\
\text { At-Fault } \\
\text { Incidents } \\
\left(n_{\text {L/SH }}=25\right)\end{array}$ & $\begin{array}{c}\text { Frequency of } \\
\text { LV Driver At- } \\
\text { Fault } \\
\text { Incidents } \\
\left(\mathrm{n}_{\mathrm{LV}}=117\right)\end{array}$ & $\begin{array}{c}\text { Percentage of } \\
\text { LV Driver At- } \\
\text { Fault } \\
\text { Incidents } \\
\left(n_{\mathrm{LV}}=117\right)\end{array}$ & $\begin{array}{c}\text { Frequency of } \\
\text { All Drivers } \\
\left(\mathrm{N}_{\text {Total }}=142\right)\end{array}$ & $\begin{array}{c}\text { Percentage of } \\
\text { All Drivers } \\
\left(\mathrm{N}_{\text {Total }}=142\right)\end{array}$ \\
\hline Aggressive Driving & 3 & $12 \%$ & 50 & $42.7 \%$ & 53 & $37.3 \%$ \\
\hline Driving Techniques & 8 & $32 \%$ & 26 & $22.2 \%$ & 34 & $23.9 \%$ \\
\hline Unknown & 0 & $0 \%$ & 15 & $12.8 \%$ & 15 & $10.6 \%$ \\
\hline $\begin{array}{l}\text { Purposeful Violation of } \\
\text { Traffic Laws }\end{array}$ & 2 & $8 \%$ & 5 & $4.3 \%$ & 7 & $4.9 \%$ \\
\hline Roadway Sight Distance & 0 & $0 \%$ & 7 & $6 \%$ & 7 & $4.9 \%$ \\
\hline $\begin{array}{l}\text { Driver Capabilities and } \\
\text { Limitations }\end{array}$ & 2 & $8 \%$ & 3 & $2.6 \%$ & 5 & $3.5 \%$ \\
\hline $\begin{array}{l}\text { Unfamiliar With } \\
\text { Roadway/ Traffic Pattern }\end{array}$ & 0 & $0 \%$ & 2 & $1.7 \%$ & 2 & $1.4 \%$ \\
\hline Fatigue and Drowsiness & 1 & $4 \%$ & 0 & $0 \%$ & 1 & $.7 \%$ \\
\hline $\begin{array}{l}\text { Use of Vehicle For } \\
\text { Improper Purposes }\end{array}$ & 1 & $4 \%$ & 0 & $0 \%$ & 1 & $.7 \%$ \\
\hline
\end{tabular}




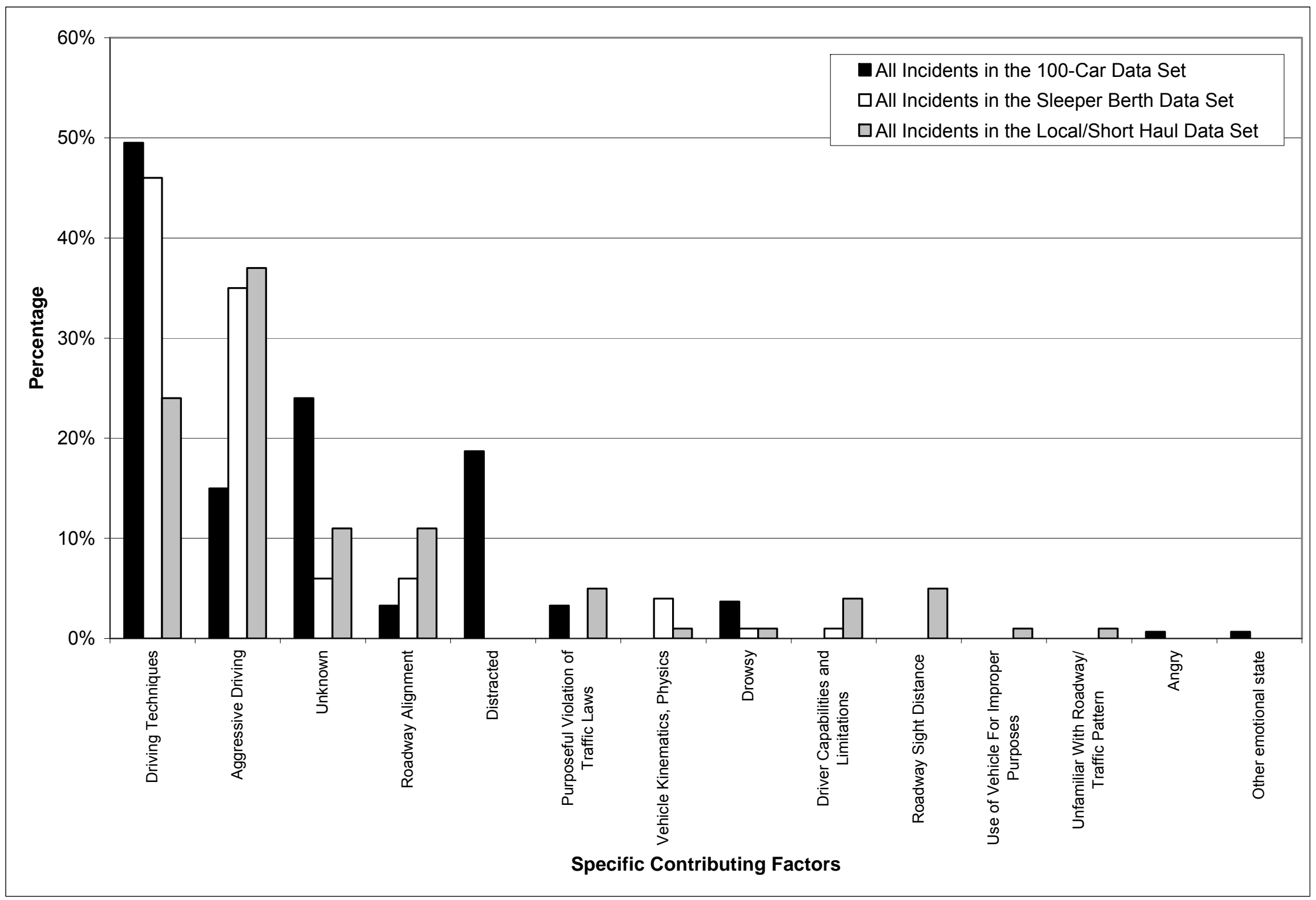

Figure 44. Percentage of Incidents in the 100-Car, SB, and L/SH Studies as a Function of Contributing Factors. 
Figure 45 shows the percentage of HV driver at-fault incidents for the 100-Car, SB, and L/SH studies as a function of Contributing Factor. The black bars in Figure 45 represent the HV driver at-fault incidents in the 100-Car Study, while the white and grey bars represent HV driver atfault incidents in the SB and L/SH studies, respectively. In the 100-Car Study, the most frequent Contributing Factors were Unknown (68.4\%), Driving Techniques (15.2\%), and Distracted (11.4\%). In the SB study, the most frequent Contributing Factors were Driving Techniques (52\%), Aggressive Driving (24\%), and Vehicle Kinematics, Physics (14\%). In the L/SH study, the most frequent Contributing Factors for HV driver at-fault incidents were Driving Techniques (32\%), Roadway Alignment (28\%), and Aggressive Driving (12\%). 


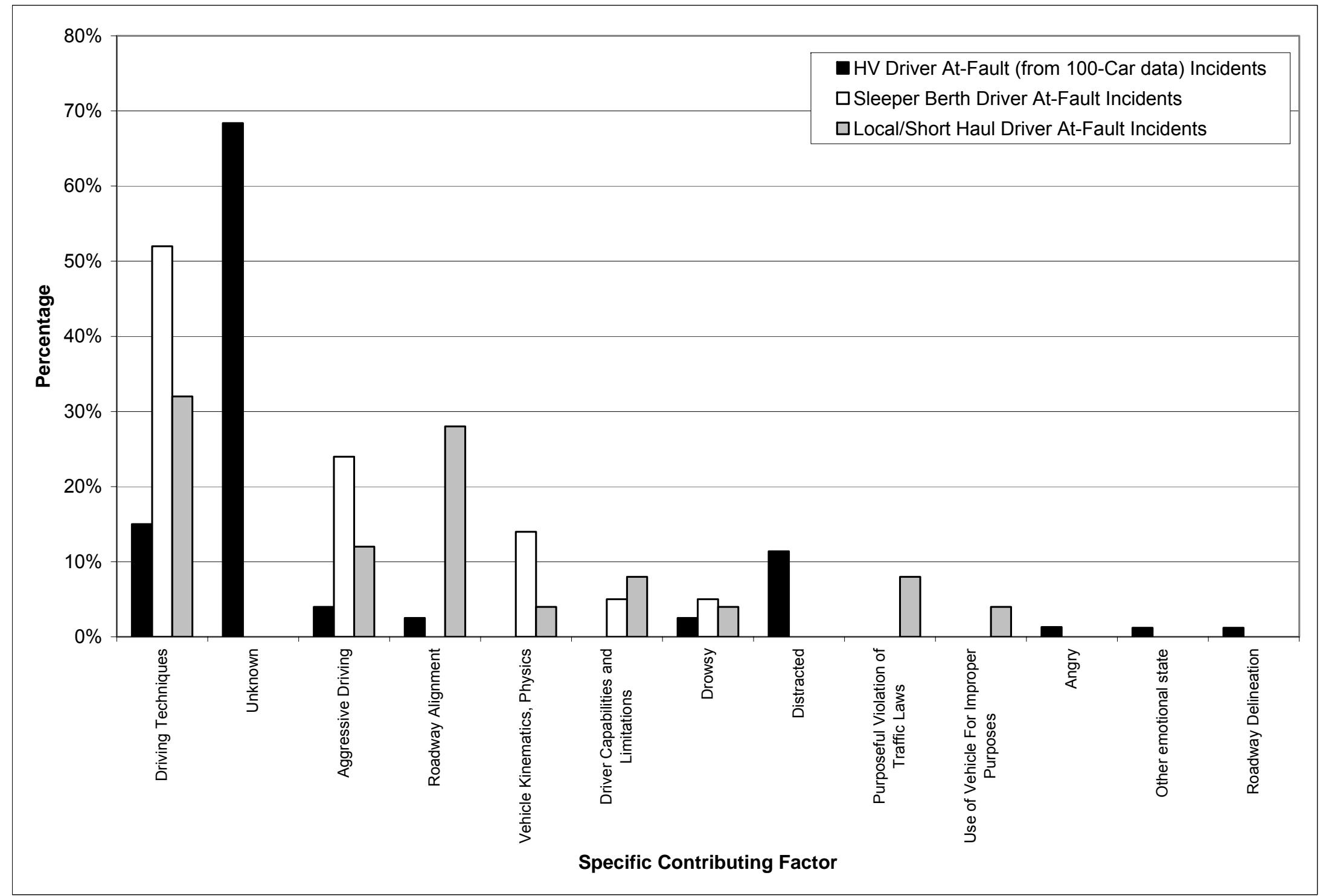

Figure 45. Percentage of HV At-Fault Incidents in the 100-Car, SB, and L/SH Studies as a Function of Contributing Factors for LV Drivers. 
Figure 46 shows the percentage of LV driver at-fault incidents in the 100-Car, SB, and L/SH studies as a function of Contributing Factor. The black bars in Figure 46 represent the LV driver at-fault incidents in the 100-Car Study, while the white and grey bars represent LV driver at-fault incidents in the SB and L/SH studies, respectively. In the 100-Car Study, the most frequent Contributing Factors for LV driver at-fault incidents were Driving Techniques (70.3\%), Distracted (22.5\%), and Aggressive Driving (22.5\%). In the SB study, the most frequent Contributing Factors for LV driver at-fault incidents were Driving Techniques (42.6\%) and Aggressive Driving (40.4\%). In the L/SH study, the most frequent Contributing Factors for LV driver at-fault incidents were Aggressive Driving (42.7\%), Driving Techniques (22.2\%), and Unknown (12.8\%). 


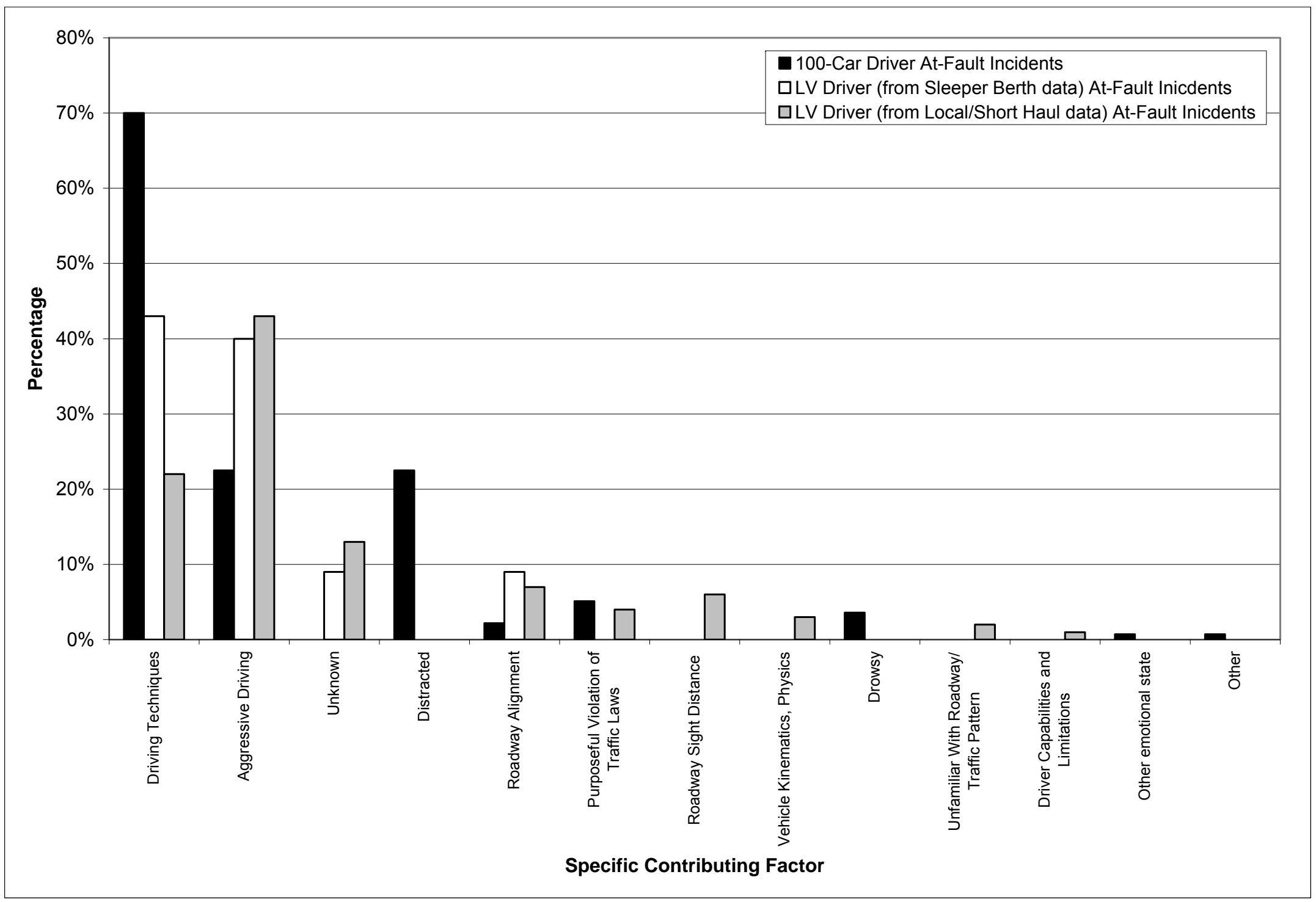

Figure 46. Percentage of LV Driver At-Fault Incidents in the 100-Car, SB, and L/SH Studies as a Function of Contributing Factor. 


\section{Summary of Contributing Factors Across Studies}

Across the three studies, the most frequent Contributing Factors were Driving Techniques (41\%), Aggressive Driving (24.1\%), and Unknown (17.1\%). As can be seen in Figures 44-46, the Contributing Factors were relatively the same depending on whether the LV or HV was at-fault.

As can be seen in Figure 45, the most frequent Contributing Factors for HV driver at-fault incidents in the 100-Car Study were Unknown (68.4\%), Driving Techniques (15.2\%), and Distracted (11.4\%). In the SB study, the most frequent Contributing Factors were Driving Techniques (52\%), Aggressive Driving (24\%), and Vehicle Kinematics, Physics (14\%). In the L/SH study, the most frequent Contributing Factors were Driving Techniques (32\%), Roadway Alignment (28\%), and Aggressive Driving (12\%). From the data it appears that the majority of at-fault $\mathrm{HV}$ drivers were classified with poor driving techniques.

It is important to note that the $\mathrm{HV}$ driver at-fault Contributing Factors in both the SB and $\mathrm{L} / \mathrm{SH}$ studies are relatively the same, yet, different from the HV driver at-fault Contributing Factors in the 100-Car Study. The reader should bear in mind that the Contributing Factor was coded with respect to the instrumented vehicle, thus, the high frequency of Unknown Contributing Factors in the 100-Car Study. If a LV driver was not at-fault, it was unlikely they would be coded with a Contributing Factor in the LV-HV interaction (as supported by the data in the 100-Car Study).

As can be seen in Figure 46, the most frequent Contributing Factors for LV driver at-fault incidents in the 100-Car Study were Driving Techniques (70.3\%), Distracted (22.5\%), and Aggressive Driving (22.5\%). In the SB study, the most frequent Contributing Factors for LV driver at-fault incidents were Aggressive Driving (42.6\%) and Driving Techniques (40.4\%). In the L/SH study, the most frequent Contributing Factors for LV driver at-fault incidents were Aggressive Driving (42.7\%), Diving Techniques (22.2\%), and Unknown (12.8\%). From the data it appears that the majority of at-fault LV drivers were classified with poor driving techniques and aggressive driving.

Recall that Hankey et al. (1999) found that $77 \%$ of crashes in the Pennsylvania crash database from 1995-1996 were cited with "human error" as the primary factor in the crash. By adding the frequency of Contributing Factors associated with human error in the three studies, a total of 294 incidents out of 427, or $68.9 \%$, of the LV-HV interactions had at least one human error as a Contributing Factor (excluding the Unknown at-fault incident in the 100-Car Study).

Yet, there were differences when only $\mathrm{HV}$ and LV driver at-fault incidents were considered. For example, $35.2 \%$ of the HV driver at-fault incidents across the 100-Car, SB, and L/SH studies had at least one human error Contributing Factor (for either driver), while $82.8 \%$ of the LV driver atfault incidents across the 100-Car, SB, and L/SH studies had at least one human error

Contributing Factor. Thus, LV driver at-fault incidents were found to be similar to what Hankey et al. (1999) found when they analyzed the Pennsylvania crash database. But, it also suggests that at-fault LV drivers were more likely than at-fault HV drivers to be coded with "human error" as a Contributing Factor in the LV-HV interactions. As the majority of crashes involve LVs rather than HVs (NHTSA, 2004), it is not all that surprising that LV drivers were more similar than HV drivers when comparing the results of the $100-\mathrm{Car}, \mathrm{SB}$, and L/SH studies to the Hankey et al. (1999) study. 
However, the Hankey et al. (199) study looked at all types of crashes. Stuster (1999) assessed driver-related factors in only LV-HV fatal crashes and found that $67.7 \%$ of LVs were cited with the driver-related factors similar to the Willful Behavior and Driver Proficiency Contributing Factor categories related to human error, while $23.8 \%$ of HVs were cited with the same driverrelated factors. These results have a similar ratio to the LV (84.1\%) and HV (33.6\%) at-fault incidents attributed to human error across the 100-Car, SB, and L/SH studies.

Hankey et al. (1999) also conducted analysis with the 1996 FARS database. They found that $31.1 \%$ of the fatal crashes involved aggressive driving as a primary factor. This is relatively similar (within 10\%) to what was found across the 100-Car, SB, and L/SH studies, where 24.1\% of the LV-HV interactions were coded with the Aggressive Driving Contributing Factors.

Across the 100-Car, SB, and L/SH studies it was found that 33.1\% of the LV at-fault incidents were coded with the Aggressive Driving Contributing Factor, while $7.2 \%$ of the HV driver atfault incidents were coded with the same Contributing Factor (excluding the Unknown at-fault incidents). Stuster's (1999) analysis of fatal LV-HV interactions found that $2.1 \%$ of the HVs were cited with the driver-related factor "Erratic/Reckless Driving," while 4.3\% of the LVs were cited with this same driver-related factor. Thus, when comparing the same type of event (i.e., $\mathrm{LV}-\mathrm{HV}$ interaction), keeping in mind that the events were different in intensity, the current analysis resulted in different results from the Stuster (1999) findings.

\section{DISCUSSION OF THE RESULTS COMPARING THE 100-CAR, SB, AND L/SH STUDIES}

The primary aims of Chapter 2 were to combine the data from the current study with the data from the L/SH and SB studies in Hanowski, Keisler, and Wierwille (2004) to illustrate the overall crash picture, and address the limitation of having only one vehicle instrumented by assessing the differences between the three studies (i.e., are LV-HV interactions fundamentally different as a function of which vehicle is instrumented?). The results from the 100-Car Study were presented in Chapter 1, while the results from both the SB and L/SH studies can be found in Hanowski, Keisler, and Wierwille (2004).

In the 100-Car Study, a total of 246 LV-HV interactions were identified. Of these, 138 (56.1\%) and 79 (32.1\%) incidents were found to be the fault of the LV and HV drivers, respectively. In the remaining 29 (11.8\%) incidents it was unknown whether the LV or HV driver was at-fault. In the L/SH study, a total of $142 \mathrm{LV}-\mathrm{HV}$ interactions were identified. Of these, 117 (82.4\%) incidents were the fault of the LV driver, while the remaining 25 (17.6\%) incidents were the fault of the $\mathrm{HV}$ driver. In the SB study, a total of $68 \mathrm{LV}-\mathrm{HV}$ interactions were identified. Of these, 47 (69.1\%) were the fault of the LV driver, while the remaining 21 (38.9\%) were the fault of the HV driver. Taken together with the current research, these three studies consistently show that $\mathrm{LV}$ drivers appear to be responsible for the majority of LV-HV interactions. Of the $427 \mathrm{LV}-\mathrm{HV}$ incidents identified across the three studies (excluding the 29 Unknown at-fault incidents in the current study), 302 (70.7\%) were judged to have been the fault of the LV driver, while the remaining 125 (29.3\%) were the fault of the HV driver (a 2.4:1 ratio). The high ratio presented 
here emphasizes the role that LV drivers play in LV-HV interaction incidents. Given that LV drivers were more likely to have initiated an incident, it is believed that efforts at addressing the $\mathrm{LV}-\mathrm{HV}$ interaction problem should include focusing on the LV driver.

There were a number of interesting findings from the comparisons between the 100-Car, SB, and $\mathrm{L} / \mathrm{SH}$ studies. Comparisons were conducted with respect to the Incident Type, Primary Maneuver, and Contributing Factor. The Incident Type comparison indicated that Lane Change Without Sufficient Gap was the most frequent Incident Type across all three studies. A breakdown of incidents as a function of the at-fault driver showed that Lane Change Without Sufficient Gap incidents were primarily attributed to LV drivers. Critical incidents that involved a LV driver changing lanes in front of an HV, leaving the HV driver with very little headway between vehicles, were a common Incident Type that was captured in all three studies.

While the Incident Types for the LV driver at-fault incidents shared some similarities across the three studies, the Incident Types for the HV driver at-fault incident were more varied across the studies. In the 100-Car Study, $48.1 \%$ of the HV driver at-fault Incident Types included Lane Change Without Sufficient Gap and Lateral Deviation of Through Traffic. In the SB study, 71.4\% of the HV driver at-fault incidents included Late Braking for Stopped/Stopping Traffic and Following Too Closely. In the L/SH study, 48\% of the Incident Types included Roadway Entrance Without Clearance, Wide Turn Into Adjacent Lane, and Late Braking for Stopped/Stopping Traffic. One possible explanation for these differences was the predominant Road Type traveled (see Figure 38), as well as the type of trucking operations included in the SB and L/SH studies. It could be argued that the HVs in the 100-Car Study represent a more diverse population of HVs since they were not limited to $\mathrm{L} / \mathrm{SH}$ and SB trucks. In fact, as shown in Table 3 (page 15), 25 different HVs were identified as being involved in LV-HV interactions in the 100-Car Study. Thus, it is likely the results for at-fault HV drivers in the 100-Car Study might be more representative of $\mathrm{HV}$ drivers in general, while the results for $\mathrm{HV}$ drivers in the SB and $\mathrm{L} / \mathrm{SH}$ studies are more representative of drivers in those specific operations.

The Primary Maneuver comparison indicated that Changing Lanes was the most frequent Primary Maneuver across all three studies. A breakdown of incidents as a function of the at-fault driver showed that Through Traffic incidents were primarily attributed to HV drivers. Critical incidents that involved an HV driver traveling forward on the roadway or straight though an intersection were a common Primary Maneuver in the three studies. However, while Through Traffic was the most frequent Primary Maneuver for HV driver at-fault incidents in both the SB and L/SH studies, the most frequent Primary Maneuvers for $\mathrm{HV}$ driver at-fault incidents in the 100-Car Study were Changing Lanes and Crossing Over the Lane Line. This suggests that, for the general population of $\mathrm{HV}$ drivers, changing lanes and crossing the lane line are difficult maneuvers. This make intuitive sense, as HVs are likely to have blind spots that make it difficult to change lanes or attempt to change a lane.

The most predominant Primary Maneuver for LV driver at-fault incidents was Changing Lanes. While in all three studies the LV driver was likely to be coded with the Changing Lanes Primary Maneuver, there were also differences across the three studies. LV drivers in the 100-Car Study also had difficulties when they were braking or stopped. LV drivers in the SB study encountered 
difficulties in through traffic, while LV drivers in the L/SH study had difficulties when they were making left turns.

The Contributing Factors category allows researchers to describe why the incident occurred. The most frequent Contributing Factor across the three studies was Driving Techniques. A breakdown of incidents as a function of the at-fault driver showed that Driving Techniques were primarily attributed to HV drivers. Thus, when the Contributing Factor was known, this was the most frequent Contributing Factor for HV driver at-fault incidents in each of the studies.

The most frequent Contributing Factors for LV driver at-fault incidents across the three studies were Driving Techniques and Aggressive Driving. These two Contributing Factors accounted for a substantial number of the LV driver at-fault incidents across the three studies. However, a large proportion of the LV driver at-fault incidents in the 100-Car Study involved the Distracted Contributing Factor. In fact, the only time a LV driver at-fault incident was coded with the Distracted Contributing Factors was in the 100-Car Study. This is almost certainly due to the fact that the LVs in the 100-Car Study were instrumented (thereby allowing analysis of the LV drivers' behaviors while driving), while the LVs in both the SB and L/SH studies were not instrumented.

\section{Summary}

The results of the current study in conjunction with Hanowski, Keisler, and Wierwille (2004) indicated that LV-HV interactions represent a serious problem. While there were several differences across the three studies, the results consistently showed that LV drivers are more likely to be responsible for the $\mathrm{LV}-\mathrm{HV}$ interaction than $\mathrm{HV}$ drivers. It is believed that the results from the 100-Car, SB, and L/SH studies provide a more complete description of the LV-HV interaction picture. Further, the comparisons among these three studies address the limitations of not having LVs and HVs instrumented. The detailed analyses that were conducted provide insight into how this problem might be addressed. Listed below are several suggestions that should be considered for reducing LV-HV interactions:

- Addressing the LV-HV interaction problem should focus on the driving behaviors of the LV driver. The LV driver was at-fault in $70.7 \%$ of the LV-HV interaction incidents recorded across the three studies.

- The primary area for LV that should be addressed involves their driving techniques and aggressive driving behaviors. Also, the instrumented LVs in the 100-Car Study showed that distraction was a significant problem in LV-HV interactions. Thus, the three studies identified three areas for LV drivers that should be targeted: distraction, aggressive driving, and driving techniques. Also, distraction, particularly from cell phones, appears to be a much bigger problem then has been reported in previous studies (e.g., Stutts et al., 2003).

- The primary area for HV drivers that should be addressed involves driving techniques. One possible method of addressing this is through improved truck driver training programs. For example, consideration should be given to ongoing (e.g., yearly) training courses. Given the high incidence of Aggressive Driving on the part of LV drivers, one of the primary areas of focus for a truck driving training program should be on defensive driving and hazard identification. 
- Infrastructure was found to play a role in HV driver at-fault incidents in the L/SH study. Drivers and/or company dispatchers should be cognizant of problematic sections of routes, and avoid such locations to the greatest extent possible.

- Technology has progressed to the point where it is possible to collect data on almost any driving-related variable. In situ data collection is one way to study a wide range of safety-related issues in a naturalistic environment. The video and performance/behavior data collected from the 100-Car, SB, and L/SH studies have been archived and provide a rich source of information that can be used for studying critical incidents, as was the case in the current effort, or other issues that might be identified at a later time. 


\section{REFERENCES}

Blower, D. (1998). The relative contribution of truck drivers and passenger vehicle drivers to truck-passenger vehicle traffic crashes. Publication No. UMTRI-98-25. Ann Arbor, MI: University of Michigan Transportation Research Institute.

Chliaoutakis, J.E.; Demakakos, P.; Tzamalouka, G.; Bakou, V.; Koumaki, M.; Darvari, C. (2002). Aggressive Behavior While Driving as Predictor of Self-Reported Car Crashes. Journal of Safety Research, 33(4), 431-443.

Cicchetti, D.V., \& Feinstein, A.R. (1990a). High agreement but low kappa: I. The Problems of Two Paradoxes. Journal of Clinical Epidemiology, 43, 543-549.

Cicchetti, D.V., \& Feinstein, A.R. (1990b). High agreement but low kappa: II. Resolving the paradoxes. Journal of Clinical Epidemiology, 43, 551-558.

Council, F., Harkey, D., Nabors, D., Khattak, A., \& Mohamedshah, Y. (2003). An examination of 'fault,' 'unsafe driving acts,' and 'total harm' in car-truck collisions. Transportation Research Record, 1830.

Dingus, T. A., Neale, V. L., Garness, S. A., Hanowski, R. J., Keisler, A. S., Lee, S. E., Perez, M. A., Robinson, G. S., Belz, S. M., Casali, J. G., Pace-Schott, E. F., Stickgold, R. A., and Hobson, J. A. (2002). Impact of sleeper berth usage on driver fatigue: Draft final project report. Contract No. DTFH61-96-00068. Washington, DC: Federal Motor Carrier Safety Administration.

Dingus, T. A., Klauer, S.G., Neale, V. L., Petersen, A., Lee, S. E., Sudweeks, J., Perez, M. A., Hankey, J., Ramsey, D., Gupta, S., Bucher, C., Doerzaph, Z. R., and Jermeland, J. (2004). The 100-car naturalistic driving study; Phase II-Results of the 100-car field experiment. Contract No. DTNH22-00-C-07007 (Task Order No. 06). Blacksburg, VA: Virginia Tech Transportation Institute.

Federal Motor Carrier Safety Administration. (2002). Cost of large truck and bus-related crashes. Retrieved on May 10, 2005 at: http://www.fmcsa.dot.gov/documents/ab01005.pdf

Hankey, J. M., Wierwille, W. W., Cannell, W. J., Kieliszewski, C. A., Medina, A., Dingus, T. A., and Cooper, L. M. (1999, January). Identification and evaluation of driver errors: Task C report, driver error taxonomy development (Draft Technical Report). Project No. DTFH-61-97-C-00051. Blacksburg, VA: Virginia Tech, Center for Transportation Research. 
Hanowski, R. J., Keisler, A. S., and Wierwille, W. W. (2001, February). Study of light vehicleheavy vehicle interaction: Phase A. study of light vehicle-local/short haul vehicle interaction. Contract No. DTFH61-96-C-00105. Blacksburg, VA: Virginia Tech Transportation Institute.

Hanowski, R. J., Olson, R. L., Perez, M., and Dingus, T. A. (October, 2001). Driver distraction analysis on naturalistic heavy vehicle data. (Contract No. DTRS57-01-P-80378). Blacksburg, VA: Virginia Tech Transportation Institute.

Hanowski, R. J., Wierwille, W. W., Garness, S. A., and Dingus, T. A. (2000). Impact of local/short haul operations on driver fatigue: Final report. Report No. DOT-MC-00203. Washington, DC: U.S. Department of Transportation, Federal Motor Carrier Safety Administration.

Hanowski, R. J., Wierwille, W. W., Gellatly, A. G., Early, N., and Dingus, T. A. (1998). Impact of local/short haul operations on driver fatigue: Focus group summary and analysis. Report No. FWHA-MC-98-029. Washington, DC: U.S. Department of Transportation, Federal Highway Administration.

Knipling, R. R., Wang, J.S., and Yin, H.M. (1993). Rear-end crashes: problem size assessment and statistical description (Technical Report DOT HS 807 994). Washington, DC: National Highway Traffic Safety Administration.

Insurance Institute for Highway Safety. (2001). Education alone won’t make drivers safer: It won’t reduce crashes. Status Report, 36(5), 1-7.

National Highway and Transportation Safety Administration. (1987). Heavy truck safety study. Report \# DOT HS 807-109. Washington, DC: U.S. Department of Transportation.

National Highway and Transportation Safety Administration. (2003). Traffic Safety Facts 2002. Washington, DC: U.S. Department of Transportation.

National Highway and Transportation Safety Administration. (2004). Traffic Safety Facts 2003. Washington, DC: U.S. Department of Transportation.

Neale, V. L., Robinson, G. S., Belz, S. M., Christian, E. V., Casali, J. G., and Dingus, T. A. (1998, May). Impact of sleeper berth usage on driver fatigue, task 1: Analysis of trucker sleep quality. Report No. DOT-MC-00-204. Washington, DC: U.S. Department of Transportation, Office of Motor Carriers, Federal Highway Administration.

Sandberg, A. (2003). The role of IVI in highway safety. Retrieved on August 13, 2004 from: http://www.nhtsa.dot.gov/nhtsa/announce/speeches/030625Sandberg/FMCSA-IVIPresent.pdf.

Stuster, J. (1999). The unsafe driving acts acts of motorists in the vicinity of large trucks. Washington, DC: U.S. Department of Transportation, Federal Highway Administration. 
Stutts, J., Feaganes, J., Rodgman, E., Hamlett, C., Meadows, T., Reinfurt, D., Gish, K., Mercadante, M., and Staplin, L. (2003). Distractions in everyday driving. Washington, DC: AAA Foundation for Traffic Safety.

Thieriez, K., Radja, G., and Toth, G. (2002). Large Truck Crash Causation Study Interim Report (NHTSA Interim Technical Report \#DOT HS 809 527). Springfield, VA: National Center for Statistics and Analysis Advanced Research and Analysis.

U.S. Department of Transportation, National Highway Traffic Safety Administration. (2003). General Estimates System Coding and Editing Manual.

Wang, J. S., Knipling, R. R., and Blincoe, L. J. (1999). The dimensions of motor vehicle crash risk. Journal of Transportation and Statistics, 2(1).

Wierwille, W. W., Hankey, J. M., Kieliszewski, C. A., Hanowski, R. J., Medina, A., Lee, S. E., Keisler, A. S., and Dingus, T. A. (2001, April). Identification and evaluation of driver errors, task H report: Final project report. Contract No. DTFH61-97-C-00051. Blacksburg, VA: Virginia Tech Transportation Institute.

Wierwille, W. W., Hanowski, R. J., Hankey, J. M., Kieliszewski, C. A., Lee, S. E., Medina, A., Keisler, A. S., and Dingus, T. A.(August, 2002). Identification and evaluation of driver errors: Overview and recommendations. Report No. FHWA-RD-02-068. Washington, DC: Federal Highway Administration.

Wierwille, W. W., Kieliszewski, C. A., Hanowski, R. J., Keisler, A. S., and Olsen, E. C. B. (2000, July). Identification and evaluation of driver errors: Task E report, investigation of critical incidents (Draft Technical Report). Project No. DTFH-61-97-C-00051. Blacksburg, VA: Virginia Tech Transportation Institute. 
For more information on the Federal Motor Carrier Safety Administration and the Office of

U.S. Department of Transportation Federal Motor Carrier Safety Administration Research and Analysis, check our website at www.fmcsa.dot.gov.

Report No. FMCSA-RRR-06-004 